\title{
Analyzing UNC-50/GMH1 dependent membrane trafficking in yeast and $C$. elegans
}

\section{PhD Thesis}

\author{
Dissertation for the award of the degree \\ "Doctor of Philosophy naturalium" (Ph.D.) \\ (Division of Mathematics and Natural Sciences) \\ of the Georg-August-Universität Göttingen \\ within the doctoral program Grundprogramm Biologie \\ of the Georg-August University School of Science (GAUSS)
}

\section{Submitted by}

Suekyoung Jeon

\section{Born in}

Seoul, South Korea

Göttingen, 2014 


\section{Thesis Committee Members:}

\section{Prof. Dr. Ralf Heinrich}

Cellular Neurobiology, Schwann-Schleiden Research Centre, Uni Göttingen

\section{Prof. Dr. Andreas Wodarz}

Anatomy and Cell Biology, Center of Molecular Biosciences, Uni Göttingen

\section{Prof. Dr. Stefan Eimer}

Molecular Neurogenetics, European Neuroscience Institute, Uni Göttingen

Zelluläre Strukturbiologie, Centre for Biological Signalling Studies, Uni Freiburg

\section{Members of the Examination Board:}

Reviewer: Prof. Dr. Andreas Wodarz

Anatomy and Cell Biology, Center of Molecular Biosciences, Uni Göttingen

\section{Second Reviewer: Prof. Dr. Stefan Eimer}

Molecular Neurogenetics, European Neuroscience Institute, Uni Göttingen

Zelluläre Strukturbiologie, Centre for Biological Signalling Studies, Uni Freiburg

Additional Reviewer (if applicable):

\section{Further Members of the Examination Board:}

\section{Prof. Dr. Ralf Heinrich}

Cellular Neurobiology, Schwann-Schleiden Research Centre, Uni Göttingen

\section{Prof. Dr. Michael Hörner}

Cellular Neurobiology, Johann-Friedrich-Blumenbach-Institute

\section{Prof. Dr. André Fiala}

Molecular Neurobiology of Behaviour, Schwann-Schleiden Research Centre

Prof. Dr. Markus Zweckstetter

Biophysikalische Chemie, Max-Planck-Institute

Date of the oral examination: $3^{\text {rd }}$ of December 2014 


\section{TABLE OF CONTENTS}

TABLE OF CONTENTS i

LIST OF FIGURES V v

LIST OF TABLES ix

ABBREVIATIONS xi

ACKNOWLEDGEMENTS Xiv

I. ABSTRACT 1

II. INTRODUCTION

2.1 Intracellular trafficking pathways in eukaryotic cells 3

2.1.1 Secretory pathway 4

2.1.2 Endocytic pathway 5

$\begin{array}{ll}2.1 .3 \text { Retrograde pathway } & 7\end{array}$

2.2 Mechanisms of intracellular trafficking by coat protein complexes $\quad 8$

2.3 Bidirectional trafficking between TGNs and endosomes $\quad 11$

$\begin{array}{ll}\text { 2.3.1 The small GTPase ARF and its regulator } & 12\end{array}$

2.3.2 Retromer, GARP and COG complex 15

2.3.3 SNARE complexes 17

2.4 A conserved transmembrane protein UNC-50/Gmh1p required for $\mathrm{nAChR}$ sorting 18

2.5 Neurotransmission at neuromuscular junctions in C. elegans 20

2.6 Regulation of nicotinic acetylcholine receptors (nAChRs) in C. elegans 21

2.6.1 Two types of nAChRs against levamisole at NMJs 21

2.6.2 Assembly, trafficking and degradation of nAChRs 24

CHAPTER I

Analyzing the evolutionarily conserved function of UNC-50/GMH1 for membrane trafficking in yeast and $\boldsymbol{C}$. elegans: in regulating membrane dynamics, secretion and recycling of molecular complexes at the Golgi-endosomal compartments 
3.3 Pharmacological phenotypes of unc-50 mutants in synaptic transmission 28

3.4 Analysis of synthetic lethal interactions of the trafficking factors in the TGN 30

$\begin{array}{ll}3.5 & \text { Aims of this study }\end{array}$

$\begin{array}{ll}\text { IV. RESULTS } & 37\end{array}$

4.1 UNC-50 colocalizes with APT-9 at the trans-Golgi networks 37

4.2 Phenotypes of cellular/functional expression of UNC-50/Gmh1p in eukaryotes 39

4.2.1 Yeast growth phenotypes on non-fermentable and limited conditions 39

4.2.2 Analysis of intracellular organelle-specific markers in yeast $S$. cerevisiae 43

4.2.3 Analysis of intracellular organelle-specific markers in C. elegans 44

4.3 Morphological analysis of coelomocytes and muscles in unc-50 mutants 48

4.3.1 unc-50 mutants show relatively enlarged lysosomes in coelomocytes 48

4.3.2 Morphological analysis of the body wall muscles by HPF-EM 51

4.4 Intracellular traffic-dynamics to the vacuolar/lysosomal compartments $\quad \mathbf{5 2}$

4.4.1 Bulk-fluid phase endocytosis functions in unc-50 mutants 52

4.4.2 FM4-46 dyes reach fast to the vacuolar membranes in gmh1 mutants 53

4.5 Recycling defects of vesicle components in gmh1 mutants 57

4.5.1 SNC2-GFP localizes to the vacuolar-membranes in gmh1 mutants 57

4.5.2 Expression of TLG2-mCherry in yeast is not affected in gmhl mutants 60

4.6 Requirement of Gmh1p for the trafficking of the yeast CLC protein Gef1p 61

4.6.1 Growth phenotypes and localization of GEF1-GFP in GMH1-depleted strains $\quad 62$

4.6.2 GMH1 deletion induces low intensity of GEF1-GFP 65

4.7 Secretion defects of ssGFP from muscles in unc-50 mutants 67

4.8 Interspecies-expression of $\mathrm{gmh} 1$, a yeast and human ortholog of unc-50, does not rescue and localize in $C$. elegans

V. DISCUSSION

5.1 UNC-50/GMH1 acts as a redundant factor in retrograde transport from endosomes to the Golgi

5.2 UNC-50/GMH1 leads to enhanced recruitments to the endosome-to-lysosomal compartments $\quad 74$

5.3 Morphological analysis of coelomocytes and muscles in unc-50 mutants $\quad 74$

5.4 GMH1 may involve indirect regulation of cargo molecules $\quad 75$

5.5 The ARF-dependent cascade trafficking by UNC-50/GMH1 76

CHAPTER II

Proteomic/Metabolic analysis of the UNC-50 by stable isotope labeling of amino acids in $C$. elegans 
VI. SPECIFIC INTRODUCTION

6.1 Stable isotope labeling of amino acids in living cells (SILAC) 80

6.2 BioID to identify interacting proteins by Biotin protein ligase (BirA) 83

6.3 $\alpha$ GFP-nanobodies for functional studies in living cells $\quad 85$

$\begin{array}{ll}6.4 \text { Studying proteome in } C \text {. elegans } & 88\end{array}$

$\begin{array}{ll}6.5 \text { Aims of this study } & 90\end{array}$

$\begin{array}{ll}\text { VII. RESULTS } & 93\end{array}$

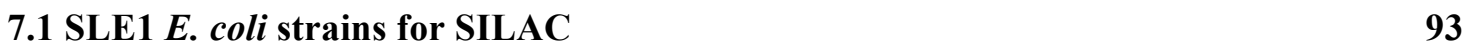

7.2 Metabolic incorporation rates of the heavy labeled-amino acids in C. elegans 94

7.3 Analysis of relative quantitative proteomes of unc-50 mutants by SILAC 98

7.3.1 Quantification of protein changes in unc-50 mutants by SILAC ratios (H/L) 99

7.3.2 SILAC proteome profiling shows trafficking factors are specifically deregulated in $\begin{array}{ll}\text { unc-50 mutants } & 101\end{array}$

7.4 Metabolic analysis of the mutants of unc-50 in C. elegans 105

7.5 Workflow strategy of SILAC-BioID in C. elegans 109

7.6 Functional specificity of the GFP nanobodies in the intracellular compartment via spatially labeling of the lumen and cytosol in C. elegans muscles 113

VIII. DISCUSSION

8.1 Metabolic SILAC labeling of proteins relies on feeding periods and concentration of the bacteria SLE1 in C. elegans

8.2 unc-50 mutants lead to protein abundance involved in the intracellular transport 121

8.3 Specificity of the anti-GFP nanobodies based on the protein structure

IX. MATERIALS AND METHODS

9.1 Materials and Reagents $\quad 126$

9.2 Methods in Molecular Biology $\quad 126$

9.2.1 Maintenance of bacterial strains 126

9.2.2 Molecular biology: PCR, Cloning and Transformation 126

$\begin{array}{ll}\text { 9.2.3 Agarose gel electrophoresis } & 127\end{array}$

$\begin{array}{ll}\text { 9.2.4 Sequencing } & 128\end{array}$

9.3 Methods used in the nematode Caenorhabditis elegans $\quad 128$

$\begin{array}{lr}\text { 9.3.1 Maintenance of strains } & 128\end{array}$

$\begin{array}{ll}\text { 9.3.2 Crossing worms } & 128\end{array}$

9.3.3 Worm PCR templates $\quad 129$

$\begin{array}{ll}\text { 9.3.4 Molecular Cloning Strategy } & 129\end{array}$

9.3.5 Transgenic lines and chromosomal integrated strains 129 
$\begin{array}{lr}\text { 9.3.6 Movement assays } & 130\end{array}$

$\begin{array}{ll}\text { 9.3.7 Pharmacological assays } & 130\end{array}$

$\begin{array}{ll}\text { 9.3.8 TR-BSA endocytosis assays } & 131\end{array}$

9.3.9 Confocal microscopy and imaging 131

$\begin{array}{ll}\text { 9.3.10 Western blots } & 132\end{array}$

9.3.11 High-pressure freeze electron microscopy (HPF-EM) 133

9.3.12 Stable isotope labeling by amino acids in C. elegans (SILAC) 133

9.3.13 Worm stocks 134

9.3.14 Integration lines by gamma irradiation of C. elegans 134

9.4. Methods used in the yeast Saccharomyces cerevisiae $\quad 135$

9.4.1 Molecular biology and strains used in yeast $\quad 135$

9.4.2 Yeast growth phenotypes 135

9.4.3 Transformation of yeast cells 135

9.4.4 Creating of knockout-gmh1 strains by PCR-based deletion cassettes 136

9.4.5 Purification of genomic DNA from yeast cells 137

$\begin{array}{ll}\text { 9.4.6 Western blotting of yeast cellular lysates } & 137\end{array}$

$\begin{array}{ll}\text { 9.4.7 Live cell Imaging } & 138\end{array}$

$\begin{array}{ll}\text { 9.4.8 Glycerol Stocks } & 139\end{array}$

X. REFERENCES 140

$\begin{array}{ll}\text { XI. APPENDIX } & 158\end{array}$

11.1 Strains of bacteria/yeast and C. elegans 158

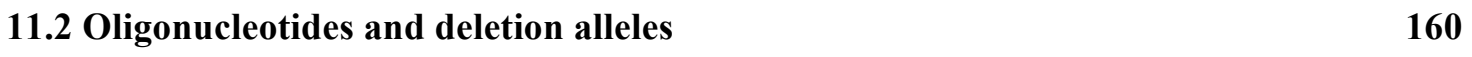

$\begin{array}{ll}\text { 11.3 Plasmids and injection constructs } & 164\end{array}$

$\begin{array}{ll}11.4 \text { Media } & 168\end{array}$

11.5 Buffers and solutions $\quad 172$

$\begin{array}{ll}\text { 11.6 Materials and reagents } & 176\end{array}$

$\begin{array}{ll}\text { 11.6.1 Research equipment used in this study } & 176\end{array}$

$\begin{array}{ll}\text { 11.6.2 Chemicals } & 178\end{array}$

$\begin{array}{ll}\text { 11.6.3 Enzymes and kits } & 180\end{array}$

$\begin{array}{ll}\text { 11.6.4 Antibodies } & 181\end{array}$

CURRICULUM VITAE 182

LIST OF PUBLICATIONS 183 


\section{LIST OF FIGURES}

Figure II.1 Overview of intracellular trafficking pathway in eukaryotic cells: Secretory pathway, endocytic pathway and retrograde pathway

Figure II.2 Three pathways of endocytosis in eukaryotic cells

Figure II.3 Transport mechanisms of coated vesicles

Figure II.4 The domain architecture of the large ARF-GEFs GBF/BIG subfamily and the consecutive action of ARF-GEFs for distinct ARFs activation in the entire sorting network

Figure II.5 Schematic representation of tethering events cooperated by GARP complex, SNAREs and Small GTPases between TGNs and carrier vesicles

Figure II.6 Schematic representation of cycling of SNARE complex in transport vesicles

Figure II.7 The evolutionarily conserved protein UNC-50 in eukaryotic systems

Figure II.8 Schematic cell organization and neuromuscular junctions (NMJs) in $C$. elegans

Figure II.9 Muscle arms at NMJs in C. elegans

Figure III.1 Intracellular trafficking routes of Lev-nAChRs by UNC-50 in the secretory pathway

Figure III.2 The similarity of HDS domains of the large ARF-GEFs in yeast, plant, nematode and human

Figure III.3 Schematic representation of cholinergic neurotransmission and pharmacological phenotypes of wild type animals on $1 \mathrm{mM}$ levamisole

Figure III.4 Pharmacological phenotypes of unc-50 mutants

Figure III.5 Schematic diagrams of research aims for understanding the intracellular trafficking of UNC-50/GMH1-dependent 
Figure IV.1 UNC-50 localizes to the trans-Golgi network and endosomal interface

Figure IV.2 Yeast growth phenotypes on synthetic complete, non-fermentable and starvation conditions

Figure IV.3 gmh1 mutants lead to enhanced trafficking to the vacuole in yeast Saccharomyces cerevisiae

Figure IV.4 unc-50 mutants show enhanced intensity of the fluorescent-tagged organelle specific markers in the Golgi-endolysosomal compartments in C. elegans

Figure IV.5 A coat protein subunit of COPI, mCherry- $\varepsilon$ COP, show enhanced intensity in unc-50 mutants in the body wall muscles, but not in recycling endosomes, tagRFPt-RAB11

Figure IV.6 unc-50 mutants show relatively enlarged lysosomes in coelomocytes

Figure IV.7 HPF-EM analysis of the organelle morphology in C. elegans muscles

Figure IV.8 Coelomocytes show normal dynamics of endocytosis by bulk-fluid phase in unc-50 (x47) mutants

Figure IV.9 Dye structure of FM 4-64 and its schematic representation of the staining pattern in yeast cells

Figure IV.10 FM 4-64 dyes are rapidly reached to the vacuolar membranes at the early time points in gmhl depletion

Figure IV.11 GFP-SNC2 appears to be staining-like on the vacuolar membrane in gmh1 mutants

Figure IV.12 mCherry-TLG2 in yeast cells does not affect in the gmh1 mutants

Figure IV.13 Cellular-overexpression phenotypes of GEF1 tagged-GFP, GFP-GEF1 and GEF1-GFP, in wild type are slightly rescued to gmh1 mutant strains

Figure IV.14 GMH1 deletion induces low intensity of GEF1-GFP, yeast CLC protein 66 
Figure IV.15 Schematic representation of endocytosis by coelomocytes of adult hermaphrocites in C. elegans

Figure IV.16 The signal sequence GFP fusion protein (ssGFP) from the body wall muscles was not properly secreted due to be trapped in the muscles in unc-50 mutants 69

Figure IV.17 Gmh1, the yeast and human ortholog of $u n c-50$ is not able to be functional in the body wall muscles in C. elegans

Figure V.1 Schematic representation of the synthetic lethality between the mutants of the unc-50/gmhl and the retrograde trafficking machineries in both yeast $S$. cerevisiae and nematode $C$. elegans

Figure V.2 UNC-50/GMH1 is a novel recycling factor in the retrograde pathway, which regulates in a redundant manner of the parallel pathway with the complex of GARP/COG/Retromer and the small GTPase RAB-6

Figure V.3 The ARF-dependent cascade transport manners by UNC-50/GMH1

Figure VI.1 Schematic workflow of stable isotope labeling by amino acids in living cells (SILAC)

Figure VI.2 Schematic representation of the enzymatic protein in vivo biotinylation mechanisms of the biotin ligase reaction

Figure VI.3 The complex structure of the antigen-GFP with the anti-GFP-nanobody fragments

Figure VI.4 Schematic diagrams of research aims for identifying and quantifying protein dynamics on UNC-50/GMH1-dependent in C. elegans

Figure VII.1 Schematic diagram of E. coli SLE1 in SILAC media for C. elegans

Figure VII.2 Stable isotope labeling by amino acids in feeding periods for C. elegans

Figure VII.3 Labeling by SILAC in wild type and unc-50 mutants

Figure VII.4 Quantitative distribution of the normalized ratios of the SILAC labeling 
Figure VII.5 Accurate quantification of practical changes of protein identified in unc-50 mutants

Figure VII.6 Functional classification of the proteins identified in unc-50 mutants 103

Figure VII.7 Schematic representation of BirA*-fusion proteins to be integrated into unc50 mutants

Figure VII.8 Functional expression of BirA*-fusion proteins in C. elegans muscles 108

Figure VII.9 Schematic workflow of SILAC-BioID in C. elegans

Figure VII.10 Analysis of the endogenously biotinylated proteins in C. elegans

Figure VII.11 Schematic representation of soluble $\alpha$ GFPNB::tagRFPt and GFP-fusion organelle specific proteins

Figure VII.12 Specificity of the $\alpha$ GFPNB to the GFP fusion proteins in the Golgi and ER

Figure VII.13 Specificity of the $\alpha$ GFPNB to the GFP/CFP fusion proteins in the mitochondria

Figure VII.14 Specificity of the $\alpha$ GFPNB to the GFP fusion proteins in the nucleus and NMJs

Figure VIII.1 Schematic specificity of $\alpha$ GFPNB complexes in the lumen and cytosol 124 


\section{LIST OF TABLES}

Table III.1 Synthetic lethal phenotypes between the trafficking factors considered functionally redundant in the Golgi-endosomes

Table VI.1 Highly conserved essential amino acids and neurotransmitters between $C$. elegans and humans

Table VII.1 Metabolic incorporation rates by SILAC labeling in generation of C. elegans

Table VII.2 Quantitative protein abundance and down-regulation of intracellular transport related factors in $u n c-50$ mutants

Table VII.3 Metabolic profiles of unc-50 mutants

Table XI.1.1 Bacterial E. coli strains used in this study

Table XI.1.2 Yeast Saccharomyces cerevisiae strains used in this study

Table XI.1.3 C. elegans strains used in this study

Table XI.2.1 DNA sequences of primers used in this study

Table XI.2.2 List of deletion alleles used in C. elegans

Table XI.2.3 Polymerase chain reaction conditions in yeast cells

Table XI.3.1 List of backbone vectors used in this study

Table XI.3.2 List of genes of interest in entry vectors

Table XI.3.3 List of genes of interest in injection vectors

Table XI.3.4 List of transgenic lines of extrachromosomal arrays (Ex.) 
Table XI.4.2 Amino acids of selective media

Table XI.5.1 List of buffers and solutions

Table XI.5.2 Antibiotic concentrations used in this study 


\section{ABBREVIATIONS}

$\Delta$

$\Delta$ Arg

$\Delta$ Lys

$\mathrm{ACh}$

AChE

$\alpha$ GFPNB

ARF

ARF-GAPs

ARF-GEFs

ARFRP

ARL

BAR

bioAMP

BioID

BirA

BPS

CBS

$\mathrm{CCV}$

CDRs

CFP

CGN

COG

COPI

COPII

DCB

DCG

DI

DIC

DTC

DTT

EE

ER

ERDA

ERGIC

ESCRT delta; null mutation

Auxotroph arginine (R)

Auxotroph lysine (K)

Acetylcholine

Acetylcolinesterase

Anti-GFP Nanobodies

ADP ribosylation factor

ARF-GTPase activating proteins

ARF-guanine nucleotide exchange factors

ARF-related protein

ARF-like

Bin/Amphiphysin/Rvs domains

Biotinyl-5'-AMP

Proximity-dependent biotinylated protein identification method

Biotin protein ligase

Batho Phenantroline diSulfate

Cystathione $\beta$-synthetase

Clathrin-coated vesicle

Complementarity determining regions

Cyan fluorescent protein

cis-Golgi network

Conserved oligomeric Golgi complex

Coated-protein complex in the Golgi-to-ER and internal Golgi transport

Coated-protein complex in the ER-to-Golgi

Dimerization and cyclophilin binding domain

Dense core granule

Disintegrin

Differential interference contrast

Distal tip cell

Dithiothreitol

Early endosome

Endoplasmic reticulum

ER-associated degradation

ER-Golgi intermediate compartment

Endosomal sorting complex required for transport 


\begin{tabular}{|c|c|}
\hline F-box & One domain of ubiquitin ligase SCF (SKP1/Cullin/F-box) complex \\
\hline GABA & Gamma-amino butyric acid \\
\hline GABARs & $\mathrm{GABA}_{\mathrm{A}}$ receptors \\
\hline GAP & GTPase activating proteins \\
\hline GARP & Golgi-associated retrograde complex \\
\hline GEF & Guanine nucleotide exchange factor \\
\hline GFP & Green fluorescence protein \\
\hline GGA & Golgi-associated, gamma-adaptin ear homology ARF-binding protein \\
\hline $\mathrm{H}-\mathrm{KR}$ & Heavy-isotope lysine and arginine \\
\hline Habc & $\mathrm{N}$-terminal regulatory domain of $t$-SNAREs \\
\hline HDS & Homology downstream of Sec7 domain \\
\hline HPF-EM & high-pressure freeze electron microscopy \\
\hline HRP & Horseradish peroxidase \\
\hline HUS & Homology upstream of Sec7 domain \\
\hline IM & Inner membrane of mitochondrion \\
\hline IMS & Intermembrane space of mitochondrion \\
\hline IPTG & Isopropyl b-o-1-thiogalactopyranoside \\
\hline ISG & Immature secreted granules \\
\hline L-KR & Light-isotope lysine and arginine \\
\hline LDLR & Low-density lipoprotein receptor \\
\hline LE & Late endosome \\
\hline Lev-nAChRs & Levamisole-sensitive nAChRs \\
\hline LGICs & Ligand-gated ion channels \\
\hline M9MM & M9 minimal medium \\
\hline MP & Metalloprotease \\
\hline MPRs & Mannose 6-phosphate receptors \\
\hline MS & Mass spectrometry \\
\hline mTOR & Mammalian target of rapamycin \\
\hline MUL & Light-isotope labeled in the mutant \\
\hline MVB & Multivesicular bodies \\
\hline N-nAChRs & Levamisole-non-sensitive nAChRs \\
\hline $\mathrm{nAChRs}$ & Nicotinic acetylcholine receptors \\
\hline NGM-N & Nitrogen-free agarose worm plates \\
\hline NLS & Nuclear localization signal \\
\hline NMJs & Neuromuscular junctions \\
\hline NSF & N-ethylmaleimide-sensitive factor \\
\hline OD & Optical density \\
\hline $\mathrm{OM}$ & Outer membrane of mitochondrion \\
\hline PCI & Proteasome, COP9 and Initiation factor-3 \\
\hline
\end{tabular}




\begin{tabular}{|c|c|}
\hline PGK & Phosphoglycerate Kinase \\
\hline PI3P & Phosphatidylinositol 3-phosphate \\
\hline PLAC & Protease and lacunin \\
\hline $\mathrm{PM}$ & Plasma membrane \\
\hline POI & Protein of interest \\
\hline PPIs & Protein-protein interactions \\
\hline PX & Phox, phagocytic oxidase domain \\
\hline RAB & Ras genes from rat brain \\
\hline $\mathrm{RE}$ & Recycling endosome \\
\hline $\mathrm{SC}$ & Synthetic complete \\
\hline SDS-PAGE & Sodium dodecyl sulfate polyacrylamide gel electrophoresis \\
\hline SILAC & Stable isotope labeling by amino acids in cell lines \\
\hline SNAREs & Soluble N-ethylmaleimide-sensitive factor attachment protein receptor \\
\hline SNX & Sorting nexin \\
\hline ssGFP & Signal sequence tagged-GFP \\
\hline STB & Shiga toxin-B-fragment \\
\hline$t$-SNAREs & Target-membrane SNAREs \\
\hline TCEP & Tris (2-carboxyethyl) phosphine hydro-chloride solution \\
\hline TfR & Transferrin receptor \\
\hline TGN & trans-Golgi network \\
\hline TIP47 & Tail-interacting protein of $47 \mathrm{kDa}$ \\
\hline $\mathrm{TM}$ & Transmembrane \\
\hline TPM & TLP18.3, Psb32 and MOLO-1 \\
\hline TPR & Tetratricopeptide repeat region \\
\hline TR-BSA & Texas-red conjugated to bovine serum albumin \\
\hline UPR & Unfolded protein response \\
\hline$v$-SNAREs & Vesicle-membrane SNAREs \\
\hline VDCs & Ventral and dorsal nerve cords \\
\hline VHS & Vps27, Hrs, and STAM \\
\hline VPS & Vacuolar protein sorting \\
\hline VTC & Vesicular-tubular cluster \\
\hline WT & Wild type \\
\hline WTH & Heavy-isotope-labeled in wild type \\
\hline WTL & Light-isotope-labeled in wild type \\
\hline YFP & Yellow fluorescent protein \\
\hline YPAD & Yeast extract peptone-dextrose \\
\hline
\end{tabular}




\section{ACKNOWLEDGEMENTS}

I would like to appreciate all those who have helped and supported me during the season of my PhD. First of all, I would like to thank my supervisor Prof. Dr. Stefan Eimer for giving me the opportunity, support and encouragement since I started my $\mathrm{PhD}$ course. For last three and a half years, I learned a lot and was able to overcome hard things without any difficulties in studying and life in Germany. For my projects, I would like to appreciate my thesis committees Prof. Dr. Ralf Heinrich and Prof. Dr. Andreas Wodarz for scientific advice and help in promoting research projects. As another my boss for my projects, I would like to express my appreciation to Prof. Dr. Blanche Schwappach for collaboration and learning opportunity in her lab with scientific advice and experience for six months. Without this help, my projects in practice would not have been well finished. In addition, I am grateful to Prof. Dr. Michael Hörner for agreeing thankfully to be an additional member of my thesis committee and Prof. Dr. Dieter Heineke for useful advice and help during the six semesters.

Through the SyMBaD international research and training $\mathrm{PhD}$ program funded by $\mathrm{EU}$ consortium (Marie Curie ITN) for three years 2011 - 2013, I had many chances to learn and travel abroad in EU countries based on the program support. I appreciate Prof. Dr. Christophe Mulle, Prof. Dr. Oliver Schlüter and Dr. Antonella Caminiti for supporting and coordinating in the program and thank you all brilliant guys SyMBaD alumnus for sharing multidisciplinary knowledge and scientific discussion in beautiful venues we had annual meetings and conferences.

I am thankful to all those who helped and supported my projects in ENI (European Neuroscience Institute) and Biochemistry I (Universitätsmedizin) in Göttingen and LIC (Life imaging center) and Core Facility of Metabolomics and Proteomics of ZBSA (Center for biological systems analysis) in Freiburg. I am especially thankful to Christian Becker, Sissi, Angelika Reichinger and Eva Helmstädter coordinating administrative supports, and grateful to Sebastian Wiese and Carsten Jäger collaborating scientific help for proteomics and metabolomics. 
Above all, I want to thank you all my colleagues so far for sharing friendship, knowledge and laboratory life. I would like to express my appreciation to all former members Sabine Koenig, Mandy Hennemann, Maike Kittelmann, Nora Wender, Nikhil Sasidharan, Katrin Schwarze, David Vasmer and Uta Wolke for all their helps and good memories in both Göttingen and Freiburg. Additionally, I am thankful to all members of Prof. Dr. Blanche Schwappach: Anne Clancy, Eric Arakel, Fabio Vilardi, Lena Musiol, Olga Lytovchenko, Markus Kilisch, Kirsten Unthan-Fechner and Gabriela Brodkorb. I was lucky and happy to have had all in the lab of Humboldtallee. Thanks a lot for sharing scientific knowledge, all materials, your kindness, consideration and Friday's cakes as well.

Now I would like to thank all brilliant and nice members Nathalie Göppert, Martin Piringer, Fenja Gawlas, Maria Deinert and Severine Kayser. I will never forget all the things for your help, friendship, team spirits and consideration during the tough seasons. I am also thankful to Dr. Oliver Kretz for nice EM pictures.

And I would like to express my appreciation to my friends Szi-chieh Yu, Hyunsim Min, Jungchul Kwon and Minsun Jung for sincere friendship and always cheering behind.

Last but not least, I would like to thank my family: dear my parents, dear Johan Dr. Sangyong Jung, dear Fr. Bartolomeo uncle \& Sr. Catherine aunt, Maria Shooshoo and Stefano Sheunghee for their constant love, devotion and support, and I dedicate my thesis to you dear my aunt who lives in heaven since last year. 


\section{ABSTRACT}

Although the basic principles of the intracellular trafficking of proteins and lipids within the secretory apparatus have been established, the precise regulation of molecular membrane transport events is still not well understood. The evolutionarily conserved Golgi membrane protein UNC-50 has been shown to be essential for cell surface expression of levamisole-sensitive acetylcholine receptors (Lev-nAChRs) in C. elegans. The yeast UNC-50 ortholog Gmh1p was demonstrated to interact with the guaninenucleotide exchange factors for ADP-ribosylation factor GTPases (ARF-GEFs) Gealp and Gea2p, which are required for ARF GTPase dependent transport vesicle budding and sorting. These data suggested that UNC-50/Gmh1p might be the membrane receptor that recruits Gea1/2p ARF-GEFs to the Golgi. However, the very mild phenotypes of unc$50 / g m h 1$ mutants yeast and $C$. elegans argue against a general transport function for UNC-50/Gmh1p.

In Chapter I, in order to find the evolutionarily conserved function of UNC-50/Gmh1p, we systematically characterized the intracellular transport unc-50/gmh1 mutants in the two model organisms the yeast $S$. cerevisiae and C. elegans. We determined that the loss of UNC-50/Gmh1p function results in an enhanced traffic-flow to lysosomes/vacuoles. Yeast gmh1 mutants display an enhanced delivery of the membrane dye FM 4-64 to the vacuole as well as recycling defects of $v$-SNAREs Snc2p back to the plasma membrane. In support of these observations, we also detected an enhanced recruitment of endolysosomal markers as well as lysosomal enlargements in unc-50/gmh1 mutants in yeast as well as in $C$. elegans. This is in agreement with a function of UNC-50/Gmh1p in endosome-to-Golgi retrograde trafficking, which has previously been shown in synthetic lethal screens. An electron microscopic analysis revealed the accumulation of large lipid filled vesicles and mitochondrial alterations in C. elegans unc-50 mutants. This further suggested that UNC-50/Gmh1p has a much broader role for cellular metabolism.

In Chapter II, we analyze UNC-50/Gmh1p's general function for cellular physiology by unbiased whole proteome as well as whole metabolome quantitative analysis. By metabolic SILAC labeling and quantitative proteomics, we identified proteins that have 
been up or down-regulated in unc-50 mutants. This analysis revealed that anterograde transport promoting proteins such as VPS-32.1, UNC-18 and GPB-1 as well as actinmyosin motility components ACT-2 and NMY-2 were up-regulated, whereas retrograde transport proteins such as dynein-RAB-6-mediated BICD-1 and lipid binding precursors VIT-3 and VIT-5 were down-regulated.

In order to identify UNC-50 interacting proteins by SILAC labeling and mass spectrometry we established the BioID method in C. elegans. This method allows to invivo biotinylate proteins in close proximity of target protein tagged with a mutated form of the bacterial biotin-ligase BirA. The BioID method allows the in-vivo labeling of transient or weak interactions that occur during intracellular trafficking events, which otherwise would be difficult to isolate by classical CoIP purifications. By expressing BirA-tagged anti-GFP-nanobodies ( $\alpha$ GFPNB) we also show that GFP-fusion protein can be tagged in-vivo, extending the usability of the BioID method. This approach using $\alpha$ GFPNB enabled us to determine the topology of GFP-tagged integral membrane proteins in-vivo. Thus, the proteomic tools established in this work will allow us to further define the UNC-50 dependent trafficking pathway at the molecular as well as cellular level. 


\section{INTRODUCTION}

\subsection{Intracellular trafficking pathways in eukaryotic cells}

In eukaryotic cells, intracellular transport of membranes and proteins is tightly regulated and highly dynamic at the same time. Intracellular membrane trafficking is mediated in large part by vesicular membrane carriers which facilitate the transport between the different intracellular organelles as well as with the plasma membrane. Three major key pathways for vesicular trafficking have evolved in eukaryotes: 1) the forward secretory pathway that delivers newly synthesized cargo to the plasma membrane, 2) the endocytic pathway that delivers cargo from the plasma membrane to the endosomal-lysosomal system, and 3) retrograde retrieval pathways that are necessary to recycle cargo and transport factors to sustain efficient sorting and transport (Fig. II.1).

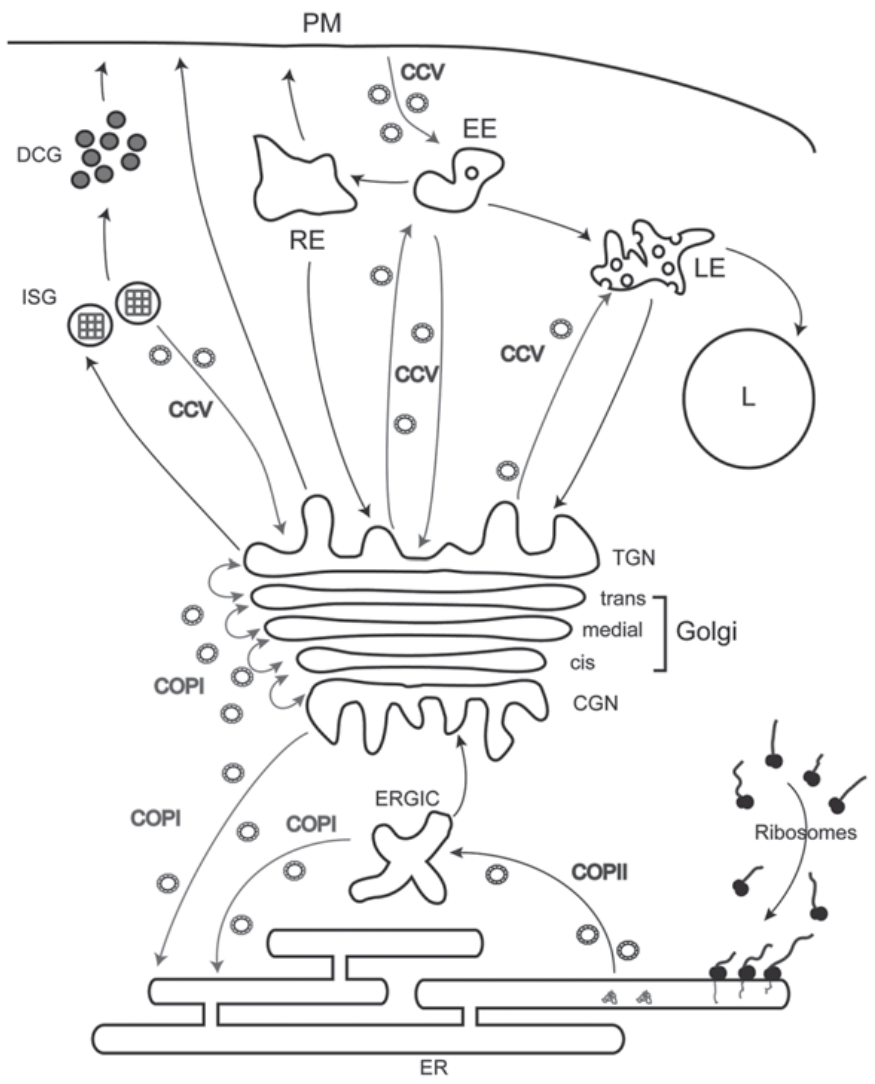

Figure II.1 Overview of intracellular trafficking pathways in eukaryotic cells. The intracellular compartments ER, ERGIC and Golgi (cis-to-trans) are major routes in the 
intracellular trafficking through the organelles endosomes-to-plasma membrane and endosomesto-lysosome. The vesicle-coated proteins mediate the membrane-bound transport in the intracellular compartments. ISG, immature secreted granules; DCG, dense core granules; PM, plasma membrane; CCV, clathrin-coated vesicle; CGN, cis-Golgi network; TGN, trans-Golgi network; RE, recycling endosome; EE, early endosome; LE, late endosome; L, lysosome; COPII, the coated-protein complex in the ER-to-Golgi; COPI, the coated-protein complex in the Golgito-ER and internal Golgi transport. Source: Picture was taken from the reference (Payne, 2009). Copyright $\mathbb{C} 2009$, Landes Bioscience

\subsubsection{Secretory pathway}

Cargo proteins that are destined to the cell surface or plasma membrane enter the secretory pathway through the endoplasmic reticulum (ER) in eukaryotic cells. They are translated directly into the ER lumen and are allowed to fold and mature in an oxidative redox environment, in contrast to the cytoplasm. Cargo proteins that leave the ER contain di-acidic motifs (e.g., Asp-X-Glu) as an ER export signal. After extensive proofreading and assessing correct folding, these di-acidic motifs are recognized by membranereceptors which sort and package the cargo proteins into COPII-coated vesicles (Teasdale and Jackson, 1996; Wang et al., 2004). These COPII coated transport vesicles subsequently bud off the ER membrane. Through lateral fusion these ER-derived transport vesicles form the vesicular-tubular cluster (VTC) or alternatively named ERGolgi intermediate compartment (ERGIC) (Appenzeller-Herzog and Hauri, 2006; Hauri et al., 2000; Schweizer et al., 1988). Subsequently the ERGIC matures further to form the first cisternae at the cis-side of the Golgi apparatus. During ERGIC maturation the COPII coat is lost as well as ER-export cargo receptors and cargo dissociate. ER-resident proteins that accidentally have escaped the ER as well as ER-export cargo receptors are recognized and retrieved back to the ER via coatomer (COPI) coated vesicles which bud off the ERGIC a subsequently fuse back with the ER (Lee et al., 2004).

After exported from the ER, cargo that stays within the ERGIC will enter the cis-side of the Golgi apparatus as the ERGIC continues to mature into the cis-Golgi by lateral fusion. The Golgi apparatus is composed of a stack of Golgi cisternae that mature from the cis- to the trans-side of the Golgi. The Golgi resident glycosylation enzymes and sorting receptors are transported in COPI coated vesicles from one Golgi cisternae in a 
retrograde manner to the following one. In contrast, the cargo always stays in the identical cisternae while the cisternae slowly matures from the cis- to the trans-Golgi (Simon, 2008). At the trans-side of the Golgi through the action of multiple small GTPases, sorting adaptors and coat proteins, the most distal Golgi cisternae is transformed into another vesicular tubular network, the trans-Golgi network (TGN). At the TGN cargo molecules are extensively sorted and packaged into cargo vesicles (e.g. secretory granules) which are selectively transported either to the plasma membrane or further within the endosomal lysosomal system (Fig. II.1 and Summarized below in the section of II.3) (Donaldson and Lippincott-Schwartz, 2000). Thus the TGN represents the central sorting station along the secretory route.

\subsubsection{Endocytic pathway}

The endocytic pathway organizes the internalization of extracellular molecules as well as lipids and membrane proteins from the plasma membrane into the cell. There are different endocytic mechanisms and several pathways to enter the endocytic pathway of a cell. First, soluble cargo proteins are recognized by specific membrane receptor (e.g. megalin) in eukaryotic cells and internalized mainly by clathrin-dependent mechanisms. Clathrin belongs to the vesicular coats that are able to be together with sorting receptors and GTPases to organize transport vesicle formation and scission (Grant and Donaldson, 2009). In contrast to clathrin-dependent endocytosis, the uptake of lipids, fluid-phase cargoes, toxins and pathogens is facilitated largely by caveolin-dependent endocytic pathways (Mayor and Pagano, 2007). Alternatively, it is the third endocytic pathway that is independent of clathrin and caveolin but requires the activation of the Arf6 GTPase. Activation of Arf6 at the plasma membrane organizes the endocytosis of glycosyl phosphatidylinositol-anchored proteins, integrins and major histocompatibility complex molecules (Gould and Lippincott-Schwartz, 2009; Grant and Donaldson, 2009; Mayor and Pagano, 2007).

The endocytosis by clathrin-coated vesicles mainly delivers cargo to the endosomallysosomal system where it is destined for degradation (Gould and Lippincott-Schwartz, 2009). Clathrin coated vesicles coming from the plasma membrane fuse with early endosomes. From early endosomes cargoes can have two possible itineraries: i) soluble 
cargo or transmembrane proteins can either recycle back to the plasma membrane through recycling endosomes or ii) be delivered to lysosomes via sorting into multivesicular bodies (MVB), late endosomes that subsequently fuse with lysosomes.

To ensure sorting, targeting and directionality during endocytic transport distinct sets of small GTPases of Rab family are critically involved in the different intracellular compartments. Rab GTPases act as molecular switches that can exist in an active GTPbound form and an inactive GDP bound form. In their active form, GTP-bound Rab GTPases bind specific effector proteins that are thus recruited to the membrane of the active Rab GTPase, inducing downstream trafficking events such as the formation of multivesicular bodies (MVBs) during late endosomal sorting by recruitment of the ESCRT complex (Fig. II.2) (Gould and Lippincott-Schwartz, 2009; Grant and Hirsh, 1999).

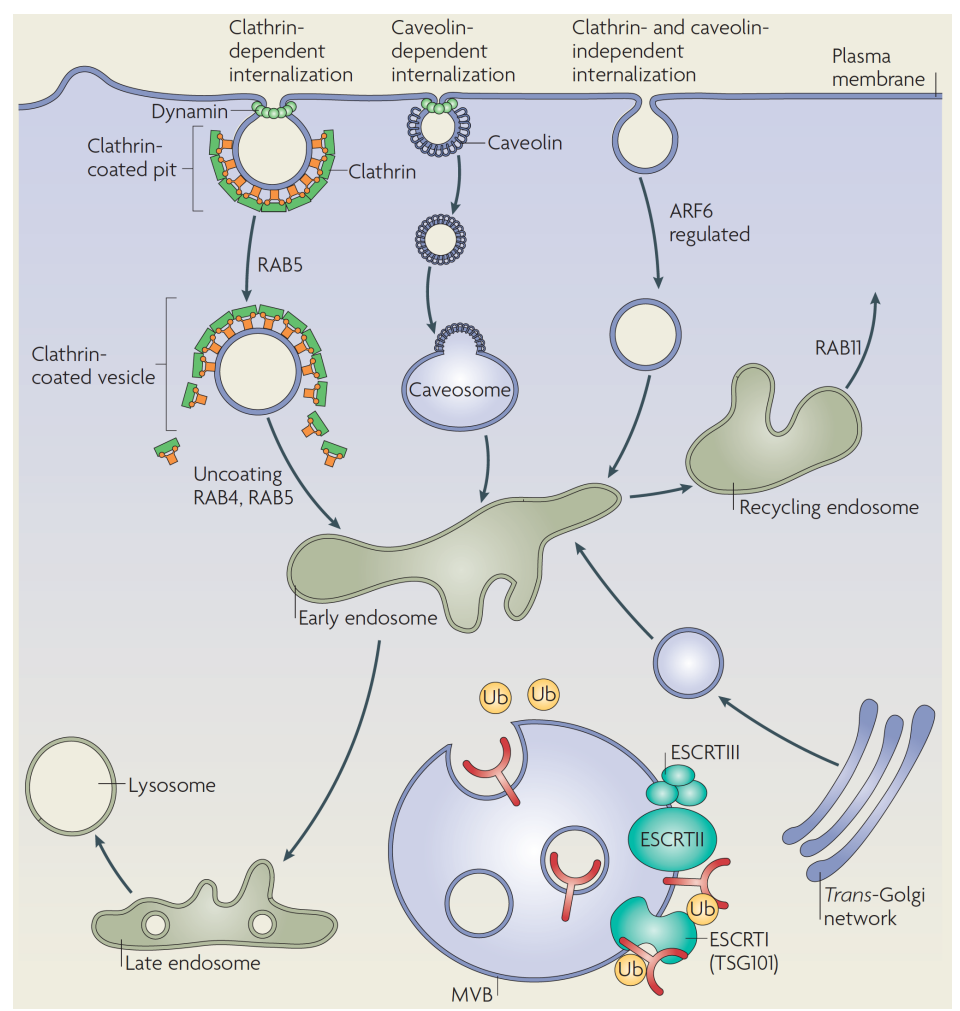

Figure II.2 Three pathways of endocytosis in eukaryotic cells. Numerous cargoes can be internalized by different mechanisms that are clathrin-dependent, caveolin-dependent and clathrin- and caveolin-independent internalization. Source: Picture was taken from the reference (Gould and Lippincott-Schwartz, 2009). 


\subsubsection{Retrograde pathway}

Intracellular vesicular trafficking between the different compartments is highly dynamic and very complex logistic. Despite multiple proofreading steps during cargo sorting into the different vesicular carriers, the trafficking process is prone to make errors leading to incorrect delivery or accidental incorporation of compartment resident proteins into transport carriers. Given that there could be errors, specific retrieval pathways are necessary for the intracellular transport, which strongly increases the efficiency of the transport processes. In terms of economic view, the recycling of cargoes and machinery components is an efficient way preventing energy waste and inappropriate protein degradation during intracellular trafficking (Donaldson and Segev, 2010; Seaman, 2005).

There are several main transport processes where recycling is of particular importance. After endocytosis at the plasma membrane particularly neurotransmitter receptors are frequently recycled and delivered back to the plasma membrane. Since the assembly, quality control and transport of multimeric receptors are inefficient, that is to say, very energy and time consuming. Therefore it is beneficial for a cell to reuse these receptors. This also allows a cell to quickly respond to different activity states that are required for membrane remodeling processes during memory formation and learning in the brain. In addition, the "Soluble N-ethylmaleimide sensitive factor (NSF) Attachment protein Receptor" (SNARE) proteins are present on the transport vesicle from stable complexes with the corresponding SNAREs on the target membrane facilitating the fusion of the vesicle with the target membrane. After the fusion event, these cis-SNARE complexes have to be unwound and dissociated from each other to allow further rounds of fusion events (Figure II.6). This is achieved by endocytic removal of the cis-SNARE complexes from the target membrane, SNARE complexes are dissociated by the ATPase NSF and the respective SNAREs are recycled back to the donor and acceptor membranes for additional rounds of fusion. In this process, Rab GTPases organize the sorting and targeting during recycling (Li et al., 2008; Sonnichsen et al., 2000); (Chen et al., 2005; de Renzis et al., 2002).

In addition, cargo receptors, which are required for cargo sorting into transport vesicles, as well as modifying enzymes such as Furin-type endopeptidases have also be retrieved in order to allow new rounds of cargo sorting and processing. After fusion of a transport 
vesicle with the target membrane cargo receptors and modifying enzymes are thus constantly recognized, packed in retrograde transport vesicles and recycled back to the donor compartment to maintain transport. A well-characterized example is the retrieval of the mannose 6-phosphate receptors (MPRs), which is required to sort lysosomal hydrolases at the TGN into transport carriers destined to lysosomes. It is bound to the tail-interacting protein TIP47 and the ubiquitous endopeptidase Furin/Kex2. They are recycled back to the Golgi from late endosomes by a retrograde pathway dependent on the Rab9 GTPase. When this retrieval pathway is blocked cargo receptors and modifying enzymes are lost to lysosomes/vacuoles (Carroll et al., 2001; Ghosh et al., 2003; Wilcox et al., 1992). Similarly, TGN38 and Shiga toxin-B-fragment (STB) follow a retrograde transport route from early endosomes to the TGN that is organized by the Rab6 GTPase (Mallard et al., 1998; Mallard et al., 2002). Several different retrograde retrieval pathways exist allowing cargo receptors and enzymes to be retrieved from various intracellular compartments in a close cooperation of the transport machinery and scaffold proteins (Reviewed by Kirchhausen, 2000).

\subsection{Mechanisms of intracellular trafficking by coat protein complexes}

Vesicular trafficking events in eukaryotic cells follow a stereotype chain of events from the donor membrane to the acceptor membrane: selection of cargo, coat assembly, vesicle budding and scission from the donor membrane, uncoating, and vesicle fusion to the target membrane (Kirchhausen, 2000). In these processes, the coat complexes COPII, COPI and Clathrin play a central role in the transport between distinct compartments ERto-Golgi, Golgi-to-ER, plasma membranes, endosomes and TGN (McMahon and Mills, 2004). Although the coat components between these transport modalities are multifarious in composition and structure, the underlying mechanisms are consistently in the same line of feature.

In the early secretory ER-to-Golgi pathway, transport vesicles are generated by the COPII coat complex, composed of the heterodimer SEC13-SEC31, SEC23-SEC24 dimer and small GTPase SAR1 (Barlowe et al., 1994; Fath et al., 2007; Kirchhausen, 2000). By contrast to the COPII coat complex, the COPI complex is responsible for the retrograde pathway from the Golgi to the ER and within the Golgi apparatus. It consists of the small 
GTPase ARF1 and the seven-coatomer subunits divided into two groups: COPI Bsubunits $\left(\alpha \mathrm{COP}, \beta^{\prime} \mathrm{COP}\right.$ and $\left.\varepsilon \mathrm{COP}\right)$ clathrin-like and COPI F-subunits ( $\delta \mathrm{COP}, \beta \mathrm{COP}$, XOP and $\zeta$ COP) a clathrin adaptor AP2-like complex (Eugster et al., 2000; Hoffman et al., 2003; McMahon and Mills, 2004). The clathrin coat complex is connected to multiple intracellular transport processes from endosomes to Golgi, Golgi to late endosomes and various endocytic pathway from the plasma membrane. The complex for clathrin coated vesicles is composed of the clathrin assembly units of heavy chains and light chains, clathrin adaptors that bind the cargo receptors and accessory proteins (McMahon and Mills, 2004). All the three coat complexes COPI, COPII and clathrin follow similar architectural principles and rules of operation concerning their regulation and coating and uncoating cycles (Fotin et al., 2004; Lee and Goldberg, 2010; Stagg et al., 2007).

Figure II.3 shows the typical steps of vesicular transport between the donor and acceptor membrane. Transport starts with the coat complex mediated trapping of cargo through its interaction with cargo receptors and subsequent budding of the donor membrane. In a concerted action of the coat complex, small GTPases of the Arf family and lipids, a transport vesicle is formed. After membrane scission the transport vesicle is transported to the acceptor membrane while the coat complexes disassemble allowing subsequent fusion of the transport vesicle with the acceptor membrane. Through multiple recognition steps the uncoated transport vesicle is tethered to the acceptor membrane. The close opposition of vesicle and acceptor membrane allows the SNAREs present on the vesicle as well as on the target membrane to zip-up. This SNARE complex formation triggers the fusion of the vesicle with the target membrane and subsequent release of the vesicular cargo into the acceptor compartment. This order of events is stereotyped for all coat complexes.

COPII and COPI coat complexes are required to generate membrane curvature that initiates the vesicle budding process. The coat protein complexes are polymerized and dissociated from the donor membrane by the small GTPase SAR1/ARF1. Thus the coat complex when the Arf-type GTPase switches back to its GDP-bound form and the uncoated transport vesicle is now able to fuse with the target membrane (Fig. II.3).

In contrast to COPI and COPII vesicles, the vesicle formation/fission of the clathrin coated complex is more complex to be involved in clathrin adaptors to gather with 
accessory proteins. In this process, the clathrin-coated vesicles (CCVs) need to be formed with the clathrin-adaptor protein complexes. To form the CCVs, the adaptor proteins such as AP-2 and alternate adaptors containing PtdIns(4,5) $\mathrm{P}_{2}$-binding sites must exist (Huang et al., 1999; Traub, 2003; Yeung et al., 1999) and other accessory proteins such as Eps15 , endophilin and amphiphysin are necessary as vesicle regulators for membrane bending and curvature in the clathrin-mediated endocytosis (Confalonieri et al., 2000; Conner and Schmid, 2003; Zhang and Zelhof, 2002). For the final formation of CCVs, the vesicle abscission requires the large GTPase dynamin to form complete clathrincoated vesicles by the GTP hydrolysis (Merrifield et al., 2005). The scission of the nascent vesicle in the clathrin system is achieved by the large GTPase dynamin, which polymerizes and forms spirals around the neck of the budding vesicle pinching it off through rounds of GTP hydrolysis. As the next process, the CCVs are finally uncoated to be fused into the endosomes (Bonifacino and Glick, 2004). The uncoating steps of the clathrin vesicles are essential to be fused to the acceptor membrane. The ATPase Hsc70 and its cofactor auxilin are involved in the dissociation of clathrin from the coat complex. The naked vesicle to be fused is guided by tethering and docking factors SNARE complex, long coiled-coil domain proteins and the membrane-associated RAB/ARF GTPases. The machinery components used in the vesicular transport are then recycled back to the original compartment through the retrograde pathway (Bonifacino and Glick, 2004; Kirchhausen, 2000). Many recycling receptors such as the transferrin receptor (TfR), low-density lipoprotein receptor (LDLR) and yolk receptor (RME-2), and their related ligands are mediated by coated vesicle complex (Grant and Hirsh, 1999; Tam, 2009; Traub, 2003). 


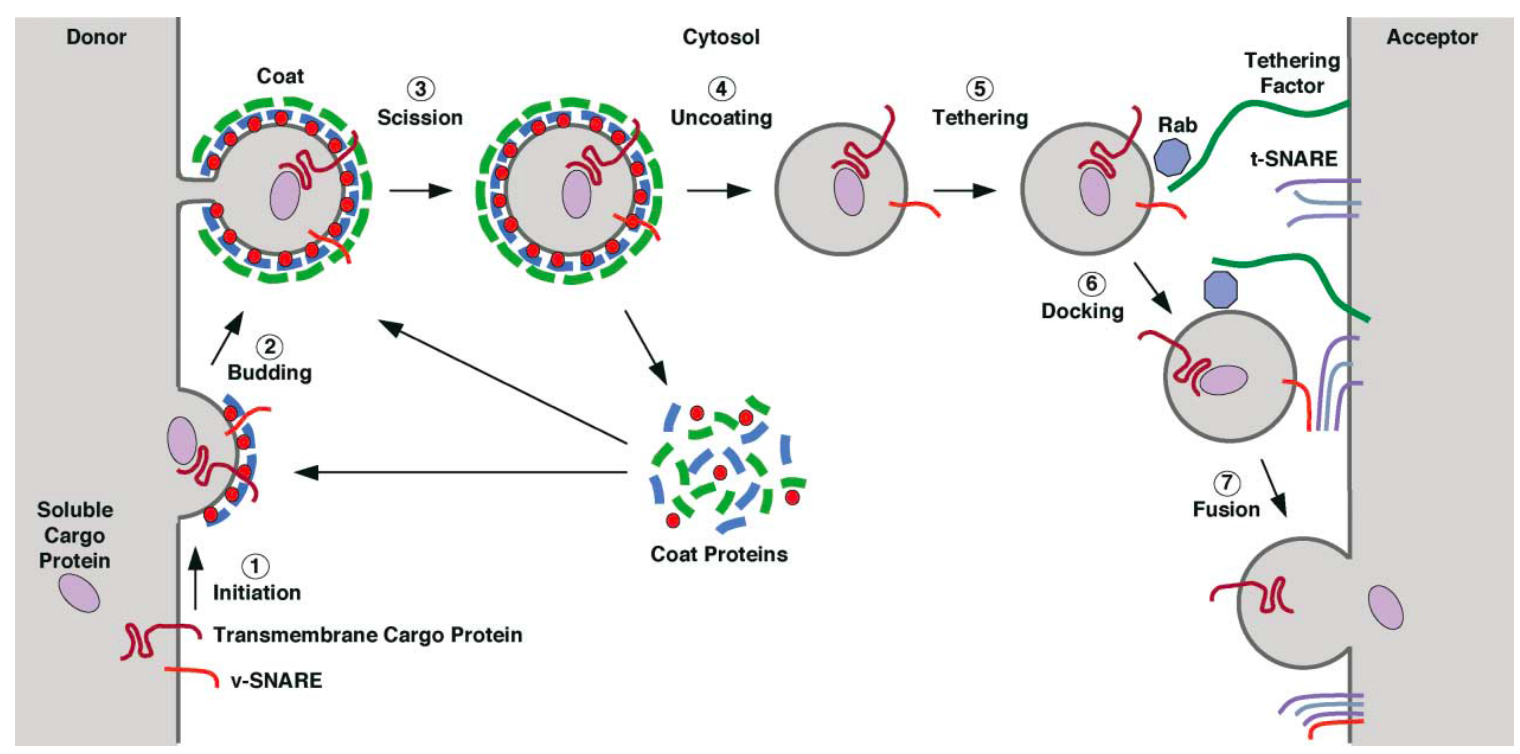

Figure II.3 Transport mechanisms of coated vesicles. The vesicle transport mediated by coat complexes require the seven steps: Initiation (Cargo selection), Budding (Coat assembly), Scission (Vesicle formation), Uncoating, Tethering, Docking and Fusion in the vesicle transport pathway mediated by COPII, COPI and clathrin. Source: Picture was taken from the reference (Bonifacino and Glick, 2004).

\subsection{Bidirectional trafficking between TGNs and endosomes}

One of the major sorting comartments within the secretory pathway is the trans-Golgi network (Camus et al., 1998; Ellgaard et al., 1999). Especially in highly polarized cells such as epithelial cells and neurons, the TGN is the main sorting hub for Apical versus basolateral sorting or sorting between axons and dendrites, respectively. In order to execute theses multiple destict sorting events the TGN uses a combinatorial set of coat proteins and small GTPases with their regulators. In order to maintain its sorting capacity, the TGN needs a specific retrieval machinery to recycle cargo-sorting receptors from endosomes or to retrieve TGN resident proteins that have accidentally left the TGN. Therefore, bi-directional transport routes between endosomes and TGN have been established, relying on the coordinated action of tethering factors, membrane fusion machinery and the small GTPase of the ARF and RAB families with its regulator (Donaldson and Segev, 2010; Rohn et al., 2000). 


\subsubsection{The small GTPase ARF and its regulator}

The ADP ribosylation factor (ARF) GTPases belong to the Ras superfamily of small GTPases that act as molecular switches cycling between a membrane-attached active, GTP-bound, form and a cytosolic-inactive, GDP-bound, form. ARF GTPases act both in anterograde as well as retrograde trafficking pathways. In contrast to Rab GTPases, the main function of ARF GTPases is to induce the budding and formation of vesicular transport carriers. To activate ARF GTPases and convert them from a cytosolic-inactive form into a membrane-bound active form the bound, GDP has to be exchanged against GTP. This ARF activation i.e. the GDP-to-GTP exchange step is triggered by membraneassociated ARF-Guanine nucleotide Exchange Factors (ARF-GEFs) containing a Sec7 domain as a signature motif. In contrast, the inactivation of ARF GTPases i.e. the hydrolysis of GTP-to-GDP is promoted by ARF-GTPase Activating Proteins (ARFGAPs) containing arginine-finger domain (Donaldson and Segev, 2010).

ARF GTPases are myristoylated at the N-terminus to promote membrane recruitments in contrast to other small GTPases that anchor to the membrane by lipid modifications at the C-terminus (Gillingham and Munro, 2007). The key to understand ARF mediated vesicular membrane transport is to understand the specific regulation of their activation and membrane recruitment by ARF-GEFs. As a membrane trafficking regulator, the ARF-GEFs consist of a conserved Sec7-domain and several non-catalytic regulatory domains: dimerization and cyclophilin binding (DCB) domain, homology upstream of Sec7 domain (HUS), guanine exchange factor or referred to Sec7 domain (GEF), homology downstream of Sec7 domain (HDS1), HDS2, HDS3 and HDS4 that are only defined by sequence homologies (Figure II.4) (Mouratou et al., 2005; Richardson et al., 2012).

All the large $(>100 \mathrm{kDa})$ Sec7-domain containing ARF-GEFs are functionally and structurally conserved in eukaryotic organisms from yeast to humans. They can be divided into two major sub-families: the GBF subfamily and BIG subfamily. The GBF subfamily includes GBF/GEA/GNOM in human, yeast and plants. The BIG subfamily consists of BIG1 and BIG2 in human and SEC7 in yeast (Donaldson and Jackson, 2000; Mouratou et al., 2005). 
Recently, the yeast large ARF-GEF Sec7p has been shown to be stably recruited to the TGN via its HDS1 domain interaction with activated-ARF1 suggesting a positive feedback mechanism of ARF activation (Richardson et al., 2012). The human ARF-GEF GBF1 is known to localize mainly at early cis-Golgi compartments for COPI complex formation required for retrograde Golgi-to-ER trafficking. However, recent studies have demonstrated that COPI is also present on the TGN domains required for the membrane recruitment of BIG1/2 GTPases (Lowery et al., 2013). These results indicate that the membrane recruitment of ARF-GEFs at the TGN is coordinated by ARF cascades similar to the well-established Rab cascades. In these ARF cascades, the early acting ARF-GEFs like GBF1 are required to activate late acting ARF-GEFs like BIG1/2 at the TGN. In addition, ARF-GEFs also interact with membrane-associated factors involved in cargospecific sorting mechanisms suggesting that vesicle formation and cargo sorting are coordinated with each other during vesicular transport (Lowery et al., 2013; Richardson et al., 2012). 
A

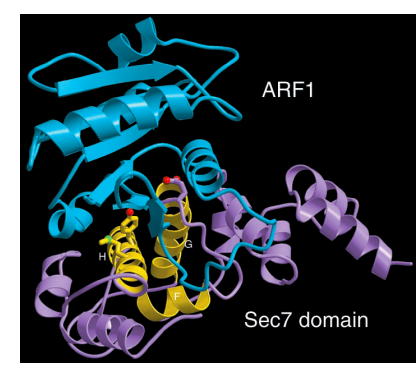

B
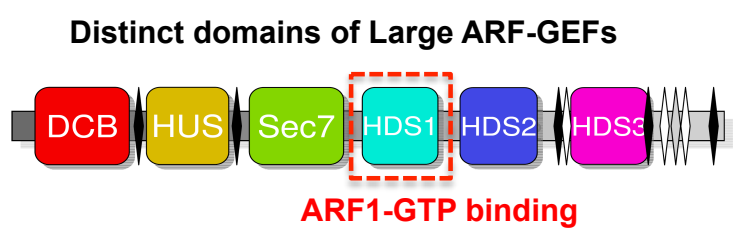

$\mathbf{N}$

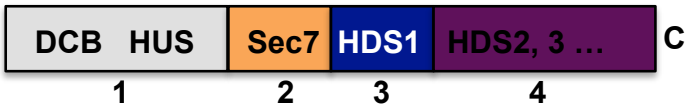

C

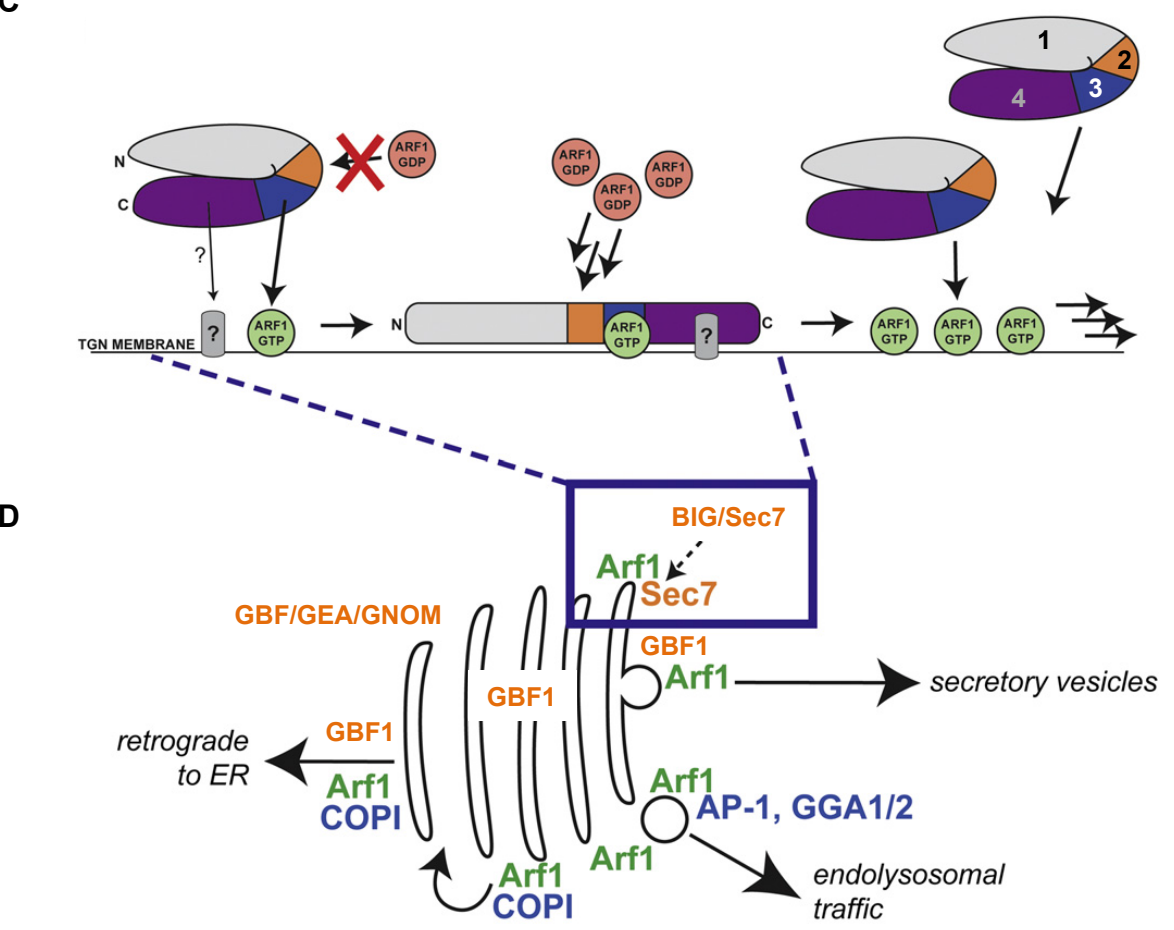

Figure II.4 The domain architecture of the large ARF-GEFs GBF/BIG subfamily and the consecutive action of ARF-GEFs for distinct ARFs activation in the entire sorting network. Structure of the Sec7 domain of the ARF-GEF GEA2 (Hydrophobic helices in yellow and essential parts for GEF activity containing a glutamic acid residue in purple) interacting with the N-terminally trimmed ARF1 (A). Schematic homology domains of large ARF-GEFs based on the model of the ARF-GEF Sec7. DCB (dimerization and cyclophilin binding domain) and HUS (homology upstream of Sec7 domain) for enhancement of GEF activity, Sec7 (guanine exchange factor conserved domain) for catalytic function of ARF-GEFs, HDS1 (homology downstream of Sec7 domain) for recruitment of the ARF-GEF SEC7 to the TGN by direct ARF1 binding, HDS2, 3 and the rest domain parts at the C-terminus of ARF-GEFs for membrane recruitment to the TGN and regulation of GEF activity (B). The HDS1 domain interaction with the ARF1-GTP and the rest of HDS domains at the C-terminus as an unknown membrane-associated additional factor 
mediates stable recruitment to the TGN (C). A model of a functional cascade action of ARFGEFs from cis-Golgi to trans-Golgi indicates in secretory pathway that early acting GBF1 activates ARFs-GTP in the cis-Golgi and the activated ARF4/5 by GBF1 recruits BIG1/2 to the TGN that mediates to the clathrin-adaptor complexes by the activation of ARF1/3 recruited by BIG1/2 of the TGN. The early acting ARF-GEFs may act a main regulator of the coated vesicle transport in secretory pathway (D). Source: A was taken from the reference (Jackson and Casanova, 2000). B was taken and modified from the reference (Mouratou et al., 2005). C and D were adapted and modified from the reference (Lowery et al., 2013; Richardson et al., 2012).

\subsubsection{Retromer, GARP and COG complex}

The mechanisms underlying the cycling of some cargo molecules are similar in eukaryotic cells and depend on the coordination of forward and retrograde vesicular trafficking (Figure II.3) (Bonifacino and Glick, 2004; Donaldson and Segev, 2010; Seaman, 2005). The retrograde transport is organized by a concerted action of several multi-subunit tethering complexes and Rab GTPases. Particularly at the TGN as the main sorting station, the need for cargo sorting-receptor retrieval is particularly high. The main retrograde trafficking complexes required for endosome-to-Golgi retrieval are the Retromer complex, Golgi-associated retrograde complex (GARP) and the conserved oligomeric Golgi (COG) complex. These complexes are cooperating with Rab GTPases and SNAREs to ensure the correct and efficient targeting of retrograde transport vesicles to the TGN (Bonifacino and Rojas, 2006). For example, the carrier vesicles containing the transmembrane manosidase phosphate receptors (MPRs) which at the TGN are required for lysosomal sorting of hydrolases are retrieved from endosomes back to the TGN. This process depends on the retromer complex which cooperates with the small GTPases RAB-5 and RAB-7 on early and late endosomes (Rojas et al., 2008). The retromer complex consists of the cargo selection complex VPS26/35/29 and the membrane structural sorting nexin (SNX) complex (VPS5 and VPS17 in yeast and SNX1 and SNX2 in mammalian). Sorting nexins contain BAR (Bin/Amphiphysin/Rvs) domains inducing membrane curvature and PX domains that bind to the phosphatidylinositol 3phosphate (PI3P) for membrane association (Seaman, 2005). The retromer complex organizes the formation of tubular sorting domains on endosomal complartments, the 
subsequent sorting of retrograde cargo into these domains and their scission to form retrograde transport vesicles.

Once these retrograde transport carriers are formed they need to be efficiently delivered to the right target membrane for subsequent fusion and cargo delivery. This step is executed by tethering complexes such as GARP and COG. These tethering complexes consist of long coiled-coil proteins and multisubunit tethering complexes which facilitate vesicle recycling by cooperation with SNAREs and small GTPases between the TGNs and carrier vesicles (Fig. II.5) (Bonifacino and Hierro, 2011; Jackson and Casanova, 2000; Lang and Jahn, 2008; Short et al., 2005). These tethering complexes are important to mediate long-range interactions to capture retrograde transport carriers at the TGN and to support correct and efficient SNARE assembly. Multi-subunit tethering complexes are programmed or activated by Rab GTPases and hold the different target and vesicular SNAREs in a fusion competent state ensuring the right stoichiometry and efficient SNARE complex formation (Conibear et al., 2003; Hayes et al., 2009; Siniossoglou and Pelham, 2001).

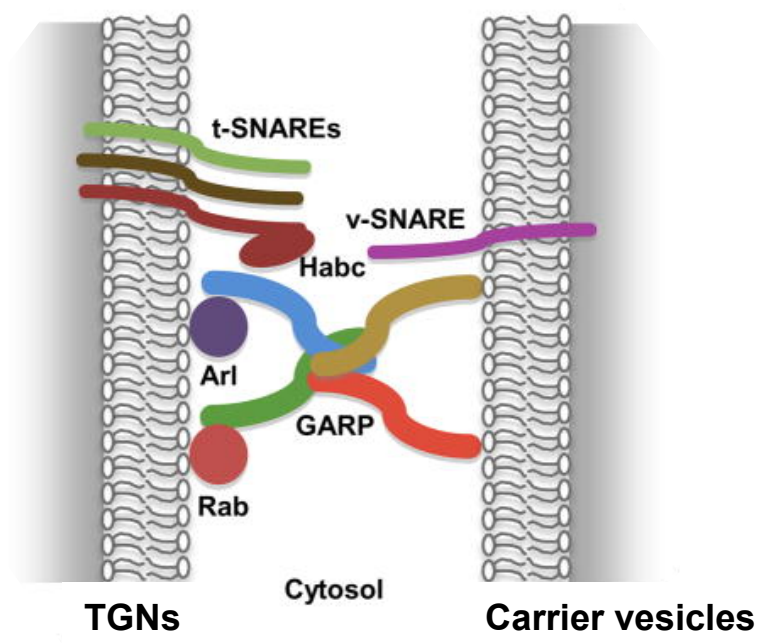

Figure II.5 Schematic representation of tethering events cooperated by GARP complex, SNAREs and Small GTPases between TGNs and carrier vesicles. A heterotetramer assembled GARP complex interacting with small GTPases RAB/ARL families binds to the Habc domain of $t$-SNAREs and is recruited to the TGN. Source: Picture was taken from the reference (Bonifacino and Hierro, 2011). 


\subsubsection{SNARE complexes}

Soluble N-ethylmaleimide-sensitive factor attachment protein receptors (SNAREs) are mostly transmembrane proteins which mediate the membrane fusion step during membrane trafficking. Since SNAREs are constantly needed to maintain vesicular trafficking, they have to be efficiently recycled via retrograde trafficking (Bonifacino and Glick, 2004). SNARE complexes form a structurally stable four- $\alpha$ helix bundle by pairing of $v$-SNARE (one $\alpha$-helix) and $t$-SNARE (three $\alpha$-helix) in a parallel manner called trans-SNARE complex. These trans-SNARE complexes hold the vesicular membrane in very close proximity to the target membrane allowing subsequent fusion of the two membranes (Bonifacino and Glick, 2004; Sutton et al., 1998). After membrane fusion all SNAREs, the target SNAREs as well as the vesicular SNAREs, are residing in the same membrane forming the so-called cis-SNARE complex. To be able to reuse the SNARE molecules for subsequent rounds of fusion, the cis-SNARE complex has to be disassembled. Through rounds of ATP hydrolysis, NSF/ $\alpha$-SNAPs proteins dissociate the cis-SNARE complex regaining the monomeric SNAREs (Fig. II.6).

In yeast, it has been shown that the $v$-SNAREs $\mathrm{SNC} 1 / 2$ are endocytosed and recycled back to the TGN after fusion with the plasma membrane. This recycling relies on retrograde tethering factors such as the GARP complex, the retromer complex and the sortin nexin VPS5 as well as on the yeast Rab6 homologue YPT6 (Galan et al., 2001; Lafourcade et al., 2004; Lewis et al., 2000; Quenneville et al., 2006). In the absence of these retrograde trafficking factors, the recycling of $v$-SNAREs SNC1/2 is defective and $\mathrm{SNC} 1 / 2$ are trafficked to the vacuole for subsequent proteolysis. 


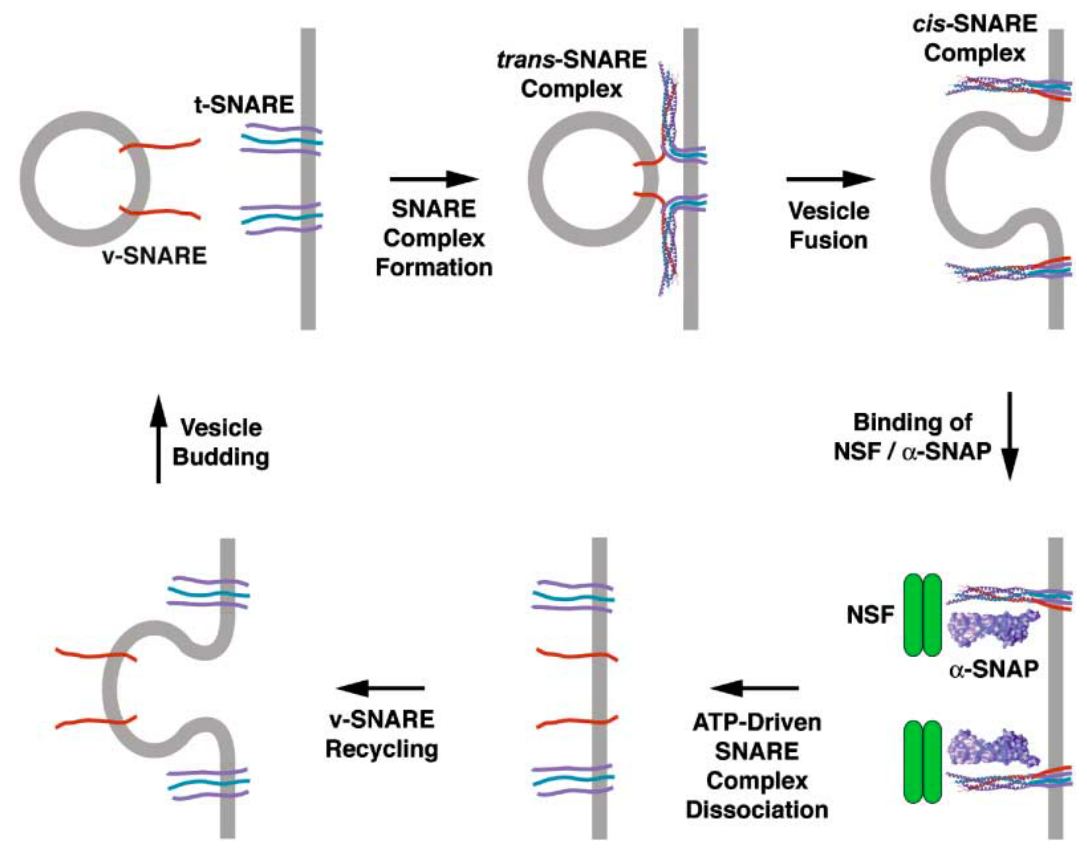

Figure II.6 Schematic representation of cycling of SNARE complex in transport vesicles. The pairing of $v$-SNARE (one $\alpha$-helix) and $t$-SNARE (three $\alpha$-helix) facilitates the membrane fusion of vesicle which is dissociated by using ATP-driven hydrolysis via binding of NSF/ $\alpha$ SNAP to the SNARE complex to be recycled in transport vesicles. Source: Picture was taken from the reference (Bonifacino and Glick, 2004).

\subsection{A conserved transmembrane protein UNC-50/Gmh1p required for $\mathrm{nAChR}$ sorting}

Assembly and transport of multimeric ion channels such as nicotinic acetylcholine receptors (nAChRs) are tightly controlled. Particularly the sorting of nAChRs is a highly dynamic process and tightly regulated by a set of regulatory proteins. Especially heteropentameric nAChRs have to be assembled from up to five different subunits. This requires a close monitoring of the assembly status, subunit composition and stoichiometry before ER exit. Similarly, at the late Golgi/TGN level, nAChRs have to be sorted and delivered to the right postsynaptic compartment. One of the factors required for nAChRs sorting at the Golgi is the integral membrane protein UNC-50 in C. elegans. UNC-50 localizes at the Golgi, which is ubiquitously expressed in C. elegans, and evolutionarily conserved from yeast to humans. UNC-50 is essential for trafficking of 
levamisole-sensitive nAChRs (Lev-nAChRs) to the cellular surface at the neuromuscular junctions (NMJs) (Fig. II.9 and III.1) (Eimer et al., 2007). Levamisole is an anthelmintic drug that acts as a nicotinic agonist and open channel blocker of a specific nAChR, the Lev-nAChR, present at NMJs. UNC-50 was first isolated from the genetic screens of levamisole resistance in C. elegans. The mutants of unc-50 show phenotypically uncoordinated movement due to cholinergic neurotransmission defects by mis-routed traffcking of the complete-assembled Lev-nAChRs in unc-50 mutants (Fig. III.1) (Eimer et al., 2007; Lewis et al., 1980). In case of the mammalian ortholog of UNC-50, named as UNCL, it has been suggested to localize in the ER and the inner nuclear membrane involved in fundamental cellular functions and maintaining of periodontal ligament fibroblasts (Fitzgerald et al., 2000; Kim et al., 2007). The yeast/human orthlogous protein is called as Gmh1p, likewise-UNC-50 of C. elegans, which interacts with the large ARFGEFs Gea1/2p of the GBF subfamily that includes GBF/GEA/GNOM in human, yeast and plant (Chantalat et al., 2003; Donaldson and Jackson, 2000; Eimer et al., 2007; Mouratou et al., 2005). The Gmhlp was initially identified as a membrane-associated suppressor of the large ARF-GEFs geal-6 in yeast Saccharomyces cerevisiae. In the study, the thermosensitive growth defects of gea mutants were appeared to be rescued by overexpression of the gmhl (Chantalat et al., 2003). This suggests that UNC-50/Gmhlp may act as a highly conserved regulator involved in the activation of the small GTP binding proteins in the intracellular trafficking in eukaryotic organisms. 

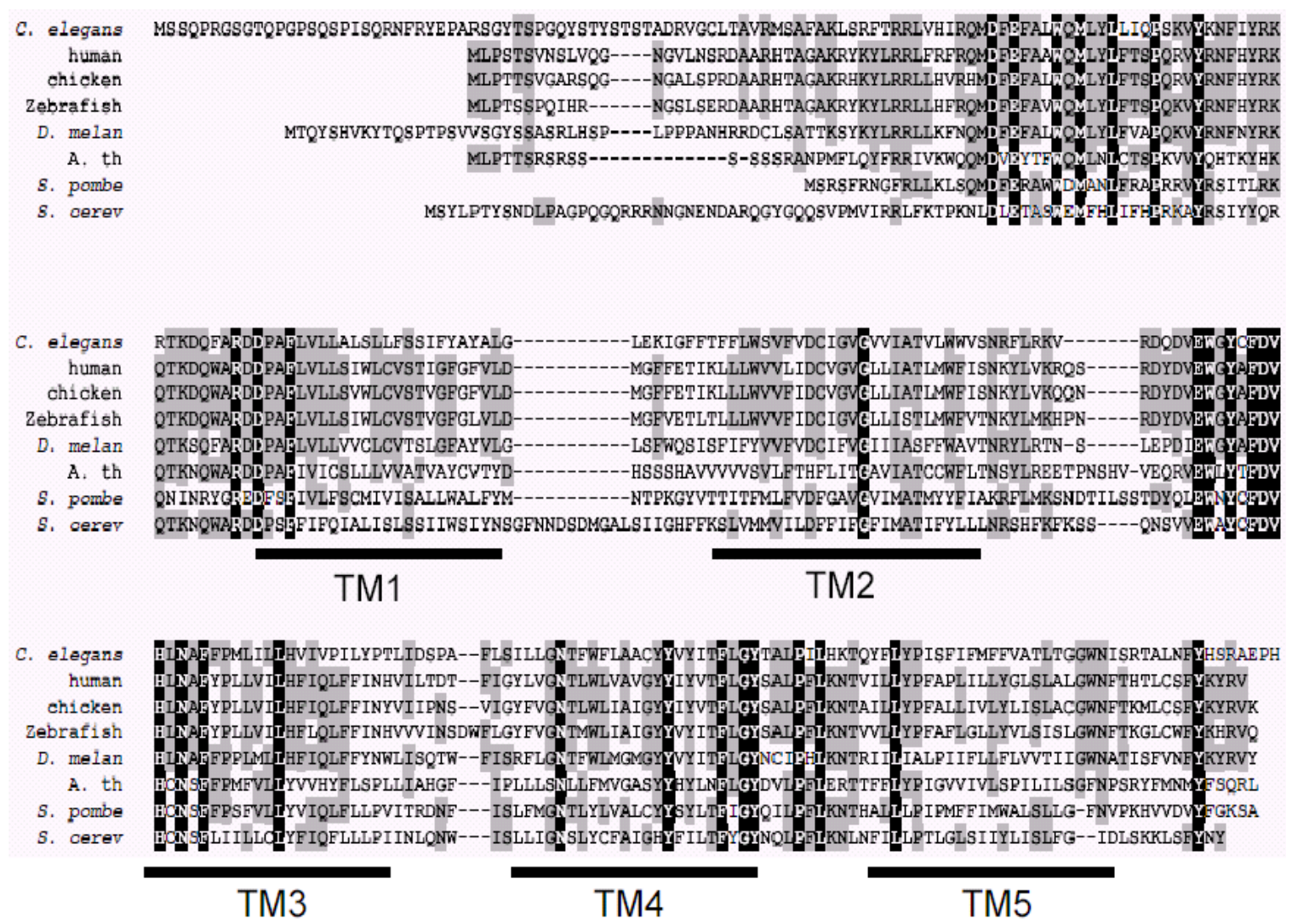

Figure II.7 The evolutionarily conserved protein UNC-50 in eukaryotic systems. From yeast to humans, UNC-50/GMH1 appears to be highly conserved with five putative transmembranes in the all species shown in the ClustalX alignment using the genomic sequence of UNC-50/GMH1. Source: Picture was provided from Stefan Eimer (Eimer et al., 2007).

\subsection{Neurotransmission at neuromuscular junctions in $C$. elegans}

At neuromuscular junctions (NMJs) in C. elegans as well as in vertebrates the neurotransmitter acetylcholine (ACh) mediates fast activatory neurotransmission (Hulme and Whitesides, 2011; Richmond and Jorgensen, 1999). Postsynaptically in bodywall muscles acetylcholine induces the opening of ligand gated ion channels of the nicotinic acetylcholine receptor $(\mathrm{nAChR})$ type. At $\mathrm{NMJs}$, the acetylcholine released from cholinergic motor neurons causes body wall muscle contraction. At the same time, the body wall muscles on the opposite side of the animal are simultaneously inhibited by release of the neurotransmitter gamma-amino butyric acid (GABA) from the inhibitory neurons. GABA activates $\mathrm{GABA}_{\mathrm{A}}$ receptors of postsynaptic membranes, which leads to 
chloride influx and muscle relaxation. As such, the coordinated reciprocal excitation and relaxation of the body wall muscle by cholinergic and GABAergic neurotransmission facilitates locomotion in C. elegans as well as other behaviors such as eating and mating (Garcia et al., 2001; Gotti and Clementi, 2004; Kim et al., 2001; McKay et al., 2004; Schuske et al., 2004; Wessler et al., 2003). In contrast to vertebrates where the motorneurons grow into the muscle fibres, C. elegans body wall muscles send out projections, so-called muscle arms, growing into the motorneuron bundles to form NMJ synapses (Fig. II.8 and Fig. II.9) (Pinan-Lucarre et al., 2014).

\subsection{Regulation of nicotinic acetylcholine receptors (nAChRs) in C. elegans}

\subsubsection{Two types of nAChRs against levamisole at NMJs}

nAChRs are highly conserved in both vertebrate and invertebrate (Touroutine et al., 2005). C. elegans NMJs contain two types of nAChRs at the postsynaptic membranes, levamisole-sensitive nAChRs (Lev-nAChRs) and levamisole-insensitive nAChRs (NnAChRs) (Fig. II.8D left). The Lev-nAChRs are hetero-pentameric ligand-gated ion channels (LGICs) encoded by $\alpha$-subunits UNC-38, UNC-63 and LEV-8 and non$\alpha$ subunits UNC-29 and LEV-1 (Lewis et al., 1980). In contrast, N-nAChRs are homopentameric $\alpha 7$-like nAChRs encoded by ACR-16 subunit (Brown et al., 2006; Touroutine et al., 2005). At NMJs, the two-type of nAChRs can also be distinguished by electrophysiological and pharmacological experiments (Eimer et al., 2007; Le Novere et al., 2002; Thany et al., 2007; Touroutine et al., 2005). Lev-nAChR mutants exhibit a slow and uncoordinated locomotion, while acr-16 mutant animals display only a mild locomotion defect. This suggests that the Lev-nAChR is the main nAChR used for normal locomotion (Touroutine et al., 2005). However, animals lacking both types of nAChRs at NMJs exhibit a strongly increased locomotion defect as compared to Lev$\mathrm{nAChR}$ single mutants showing that both $\mathrm{nAChR}$ are used for locomotion. Interestingly, in unc-50 mutants only the Lev-nAChR is mistrafficked and degraded while the ACR-16 type of nAChR is properly delivered to the synapse (Eimer et al., 2007). 
A

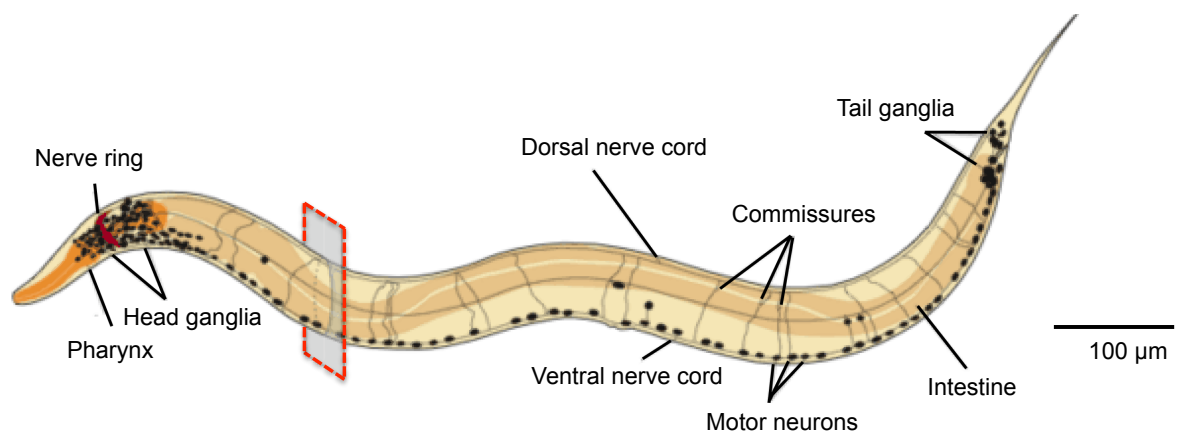

B

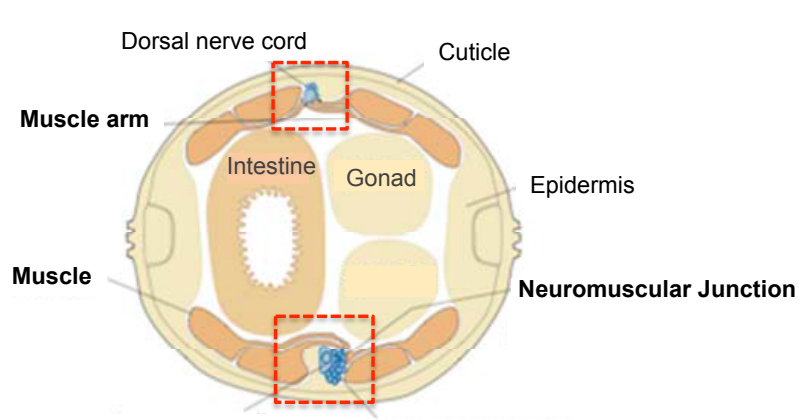

Motor neuron cell body Ventral nerve cord

Cross-sectional cut of the body $C$. elegans
C

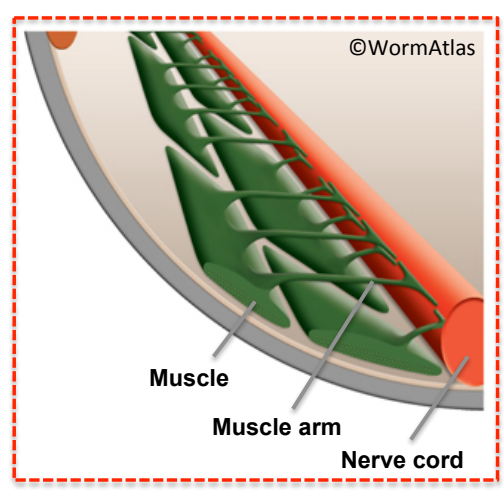

Neuromuscular Junction

D
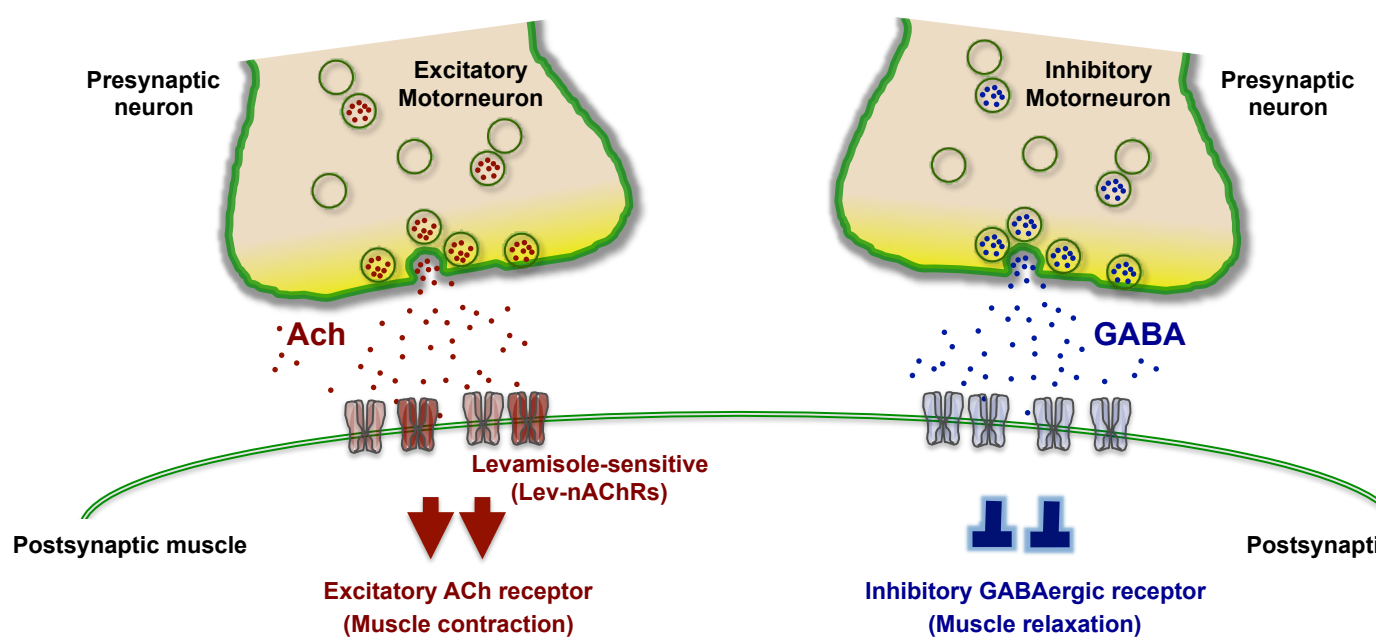

$\because \because \because$ GABA

$\therefore \therefore \therefore \therefore$

ascos of
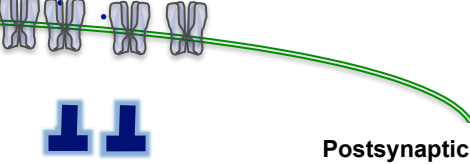

Postsynaptic muscle

Inhibitory GABAergic receptor

(Muscle relaxation)

Neuromuscular Junctions (NMJs) of C. elegans

Figure II.8 Schematic cell organization and neuromuscular junctions (NMJs) in C. elegans.

Adult hermaphrodites consist of 302 neurons and 95 muscles in C. elegans, a nice model system to study synaptic transmission, protein function and intracellular transport as a eukaryote system, in which both excitatory and inhibitory innervations exist. As a simple organism of $1 \mathrm{~mm}$ transparent roundworm, the body organization of C. elegans represents (A). It has organs, 
differentiated tissues and innervation system along with the nerve cords, the dorsal and the ventral containing motor neurons. The body wall muscles are composed of quadrants along the sides of ventral and dorsal nerve cords (VDCs) (B). Red dashed boxes in order (A-C) show crosssectional cut of the worm body indicating that the VDCs form functional synapses with the body wall muscles nearby by stretching muscle arms to the nerve cords (C). The excitatory and inhibitory innervations at the NMJs are mediated by two-type nAChRs (levamisole-sensitive and non-sensitive receptors) and $\mathrm{GABA}_{\mathrm{A}}$ receptors, respectively, by which the behaviors such as the locomotion of the sine-wave movement forward/backward of the worm are balanced by muscle contraction and relaxation at the NMJs (D). Source: A and B were provided from Stefan Eimer, C was taken from WormAtlas (Altun, 2002-2012).

A

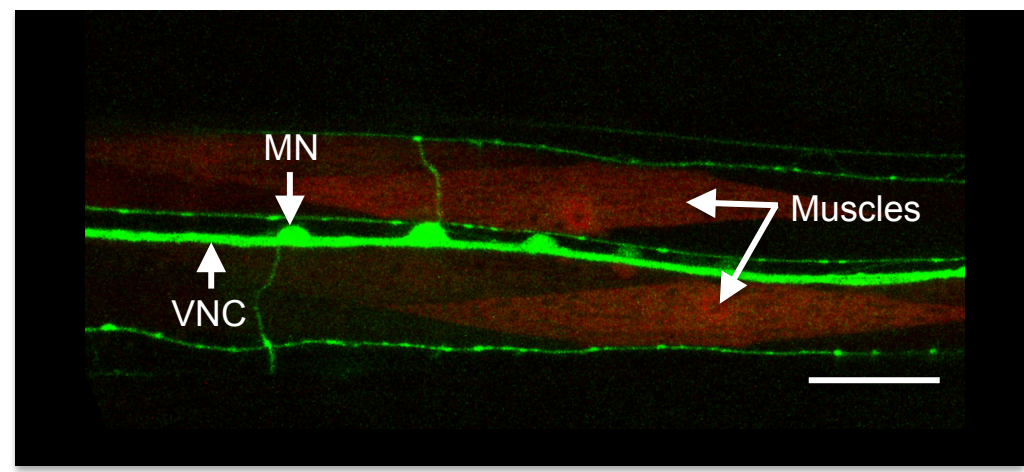

B

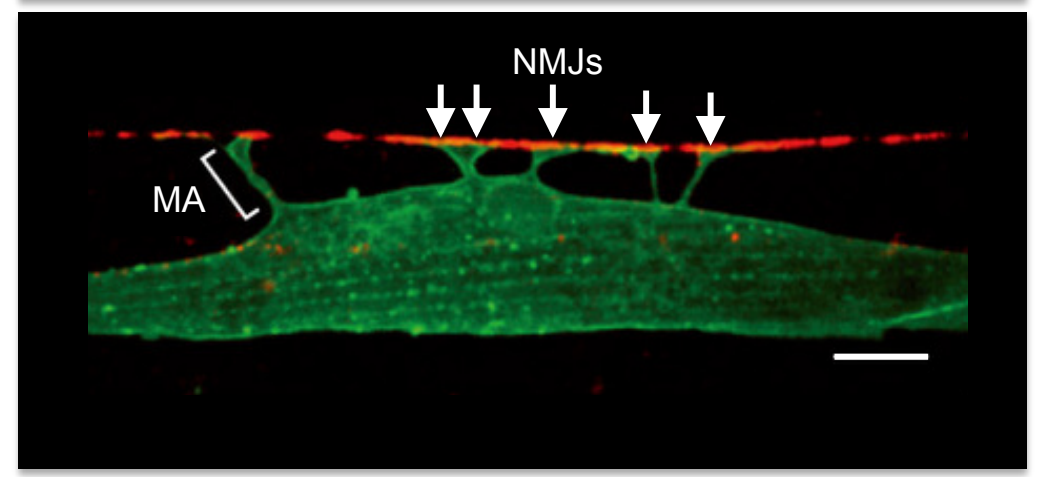

Figure II.9 Muscle arms at NMJs in C. elegans. In wild type, the body wall muscles (in red) along with the ventral nervous cord (VNC in green) containing the motor neurons (MN) were shown by using the strain sjEx [InJ13;InJ64] expressing green fluorescent-fusion protein (GFP) in neurons (prab-3::gfp) and red fluorescent-fusion protein in muscles (pmyo-3::tagRFPt) (A). A receptor subunit, UNC-29-RFP, of Lev-nAChRs is shown to cluster at the postsynaptic membrane at the NMJs. The muscle arms (MA) in green are projected out to the synaptic terminal of the motor neurons (B). Scale bars indicate $20 \mu \mathrm{m}$ (A) and $10 \mu \mathrm{m}$ (B), respectively. Source: B was taken from the reference (Pinan-Lucarre et al., 2014). 


\subsubsection{Assembly, trafficking and degradation of nAChRs}

nAChR pentamers are synthesized and assembled in the endoplasmic reticulum (ER) and transported through the Golgi apparatus and TGN en route to the plasma membrane (Camus et al., 1998; Ellgaard et al., 1999; Green and Millar, 1995; Richard et al., 2013; Smith et al., 1987). During their biosynthesis in the ER, nAChRs are tightly regulated in their folding, assembly and maturation by multiple factors such as the chaperones BiP, calnexin, RIC-3 and ER membrane protein complex (EMC)-6 (Forsayeth et al., 1992; Gelman et al., 1995; Halevi et al., 2002; Richard et al., 2013). The nAChR folding and assembly have been shown to be very inefficient due to the degradation of a large part of nAChRs by the ER-associated degradation (ERAD) machinery (Christianson and Green, 2004; Richard et al., 2013; Wanamaker et al., 2003; Wang et al., 2002). In mouse muscle cells, the nAChR that failed to assemble properly in the ER are rapidly degraded and only about $30 \%$ of newly synthesized subunits reach the cell surface, indicating that nAChRs undergo tight regulation in the ER (Merlie and Lindstrom, 1983). For example, the unassembled subunits of $\mathrm{nAChRs}$ containing retention signals $\mathrm{PL}(\mathrm{Y} / \mathrm{F})(\mathrm{F} / \mathrm{Y}) \mathrm{XXN}$ of the first transmembrane (TM1) fragment are retained in the ER. In case of the exported receptors out of the ER, a conserved $\mathrm{Arg}^{313}-\mathrm{Lys}^{314} \mathrm{ER}$ motif in the TM3-TM4 is present for COPI-associated transport in the unassembled $\alpha$-subunits of nAChRs (Keller et al., 2001; Ren et al., 2005).

After passing the ER quality control, functionally assembled nAChRs are delivered to the Golgi apparatus where they are sorted to the synapse (Ellgaard et al., 1999; Gu et al., 2001; Rohn et al., 2000; Rouille et al., 2000; Zerangue et al., 1999). In order to maintain the efficient expression of the assembled Lev-nAChRs in vivo, the auxiliary proteins UNC-50, UNC-74 and RIC-3 are required for functional expression of Lev-nAChRs in the plasma membrane of Xenopus laevis oocytes (Boulin et al., 2008).

These studies indicate that multimeric protein complexes in eukaryotic cells, even though they are similar, have subtype-specific sorting and trafficking pathways (Bruneau et al., 2005; Mallet and Maxfield, 1999; Rohn et al., 2000). 


\section{CHAPTER I}

Analyzing the evolutionarily conserved function of UNC-50/GMH1 for membrane trafficking in yeast and $\boldsymbol{C}$. elegans: in regulating membrane dynamics, secretion and recycling of molecular complexes at the Golgiendosomal compartments 


\section{SPECIFIC INTRODUCTION}

\subsection{Subtype-specific sorting manners of nAChRs by UNC-50}

UNC-50 deficiency causes transport of Lev-nAChRs to the lysosome (Eimer et al., 2007). Consequently, the complex of Lev-nAChRs then does not arrive at the postsynaptic membrane (Fig. III.1). The mutants of unc-50 in C. elegans show strong levamisole resistance and locomotion defects, but are mostly fine for normal growth. These phenotypes support that the second type of nAChRs covers alternatively functional needs of the regular synaptic transmission to compensate for the levamosle receptor loss (Eimer et al., 2007). In contrast to the heteromeric Lev-nAChRs, trafficking of $\mathrm{N}$ $n A C h R s$ and $\mathrm{GABA}_{\mathrm{A}}$ receptors (GABARs) for inhibitory innervations are not affected in unc-50 mutants. The misfolded nAChRs and GABARs are treated by regulation of rapid ERAD through the ubiquitin-proteasome system (Christianson and Green, 2004; Eimer et al., 2007; Gallagher et al., 2007).

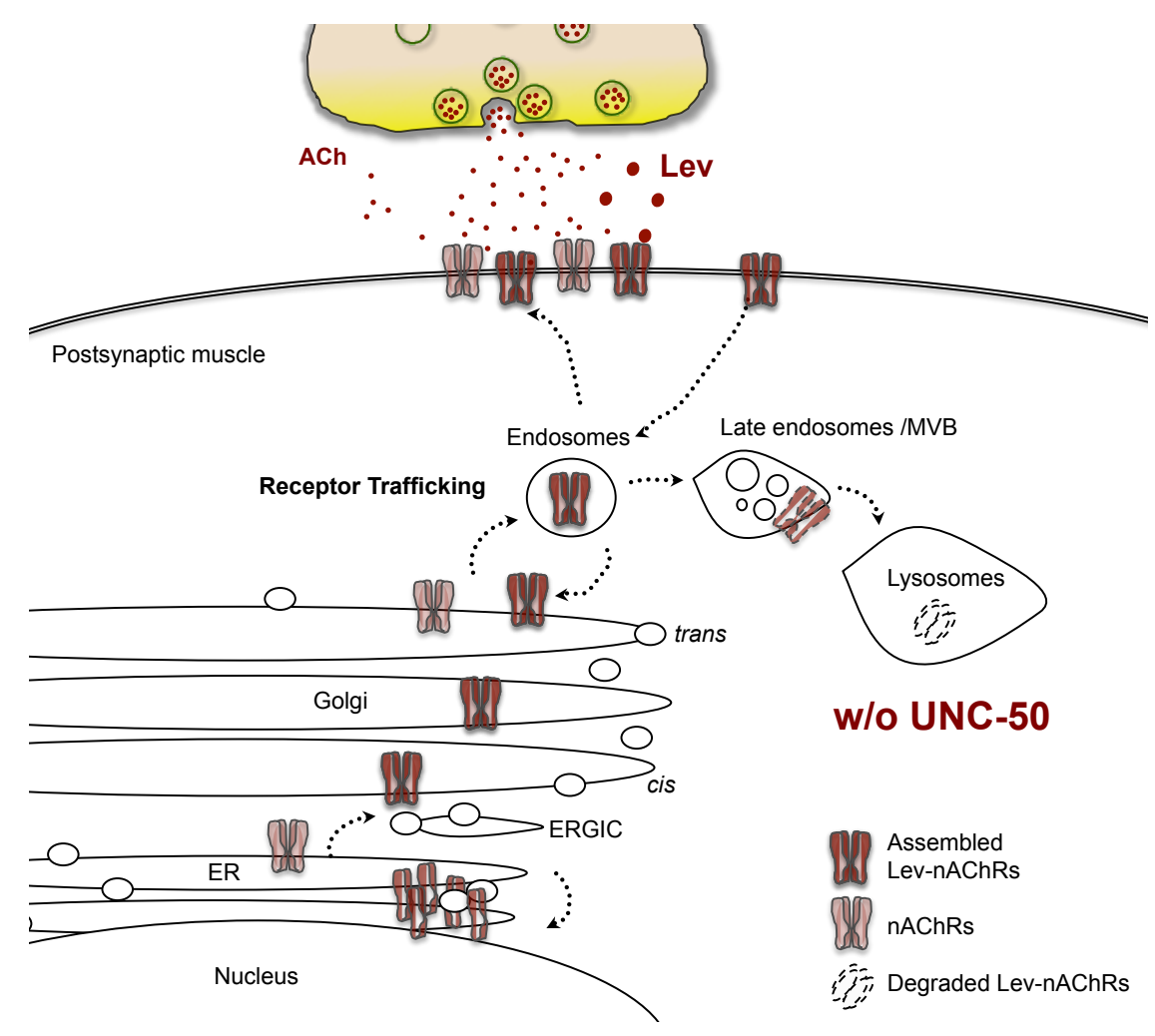


Figure III.1 Intracellular trafficking routes of Lev-nAChRs by UNC-50 in the secretory pathway. Intracellular transport of assembled Lev-nAChRs from the Golgi to the cell surface are regulated by the conserved Golgi membrane protein UNC-50 which acts as a sorting factor for subtype-specific Lev-nAChRs. In unc-50 mutants, unassembled receptors of Lev-nAChRs are retained in the ER whereas the assembled complex is delivered to the lysosome to be degraded through the UNC-50-dependent secretory pathway. Source: Picture was represented by the author based on the reference (Eimer et al., 2007).

\subsection{A transmembrane receptor GMH1 of the ARF-GEFs Gea1/2p}

Membrane-mediated cargo targeting of ARF activation is controlled by ARF-GEFs that consist of multi-domain proteins to coordinate tight regulation of membrane traffic (Chardin et al., 1996; Goldberg, 1998; Mouratou et al., 2005). As regulation mechanisms of ARF-GEFs through the multi-domains containing the catalytic Sec7-domain, it has been recently discovered that the large ARF-GEF Sec7 domain in yeast is recruited to the TGN via the HDS1-domain direct interaction with ARF1-GTP (Richardson et al., 2012). In addition to the Sec7 ARF-GEFs, the human large ARF-GEF GBF1, known as localized in the early- and internal-Golgi, has also been present in the TGN for the membrane recruitment of BIG1/2 GTPases through the distinct small GTPase-cascade activation from early-Golgi to the TGN (Lowery et al., 2013; Richardson et al., 2012). The membrane recruitment of ARF-GEFs at the TGN is dependent on the non-catalytic HDS domains by its consecutive acting from the early-to-final sorting venue. However, the ARF-GEFs-related membrane-associated factors of the membrane are little unknown that may act as critical regulators of the cargo-specific sorting routes (Lowery et al., 2013; Richardson et al., 2012). To date, UNC-50/Gmhlp has only been proposed as a transmembrane receptor to recruit ARF-GEFs Gea1/2p to the Golgi membrane since the Gmh1p was identified by Chantalat et al. in 2003. From the study, UNC-50/Gmh1p is known as a physical interaction partner of the ARF-GEFs Gea1/2p via the HDS domains at the C-terminus of Gea1/2p (Fig. III.2), and the interaction between Gmh1p and Gea1/2p has been observed not only in yeast, but also in nematode and human (Chantalat et al., 2003; Eimer et al., 2007). These data indicate that it is likely to be a conserved mechanism involved in the small GTPase ARF, its regulator ARF-GEF and a transmembrane receptor in the intracellular transport of eukaryotic organisms. 


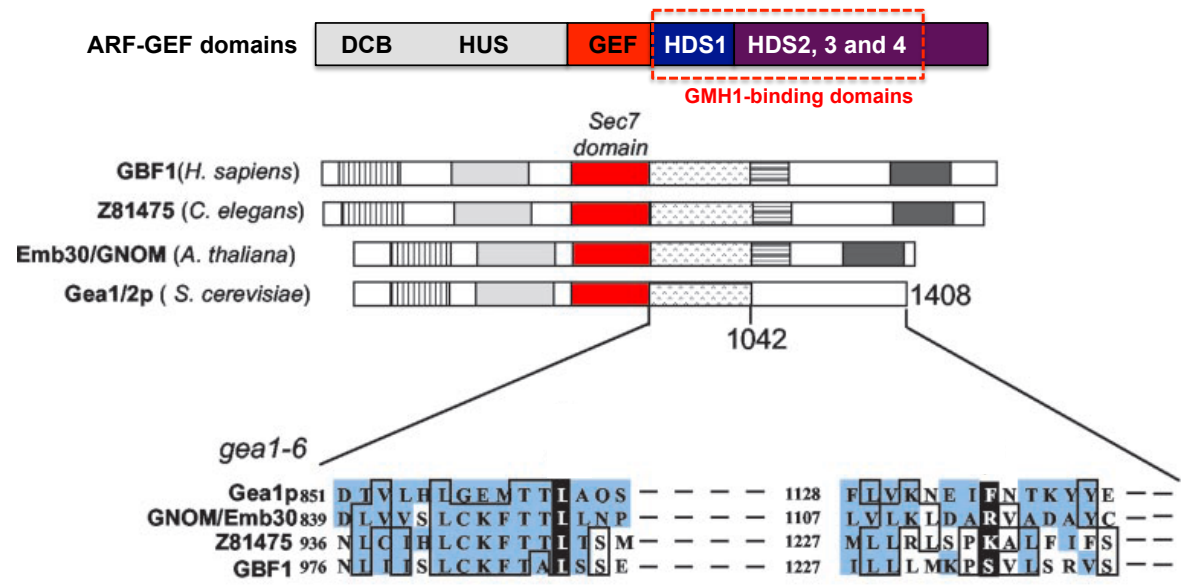

Figure III.2 The similarity of HDS domains of the large ARF-GEFs in yeast, plant, nematode and human. Schematic domains of GBF subfamily (GBF/GEA/GNOM) of the large ARF-GEFs represent (Refer to the Fig. II.4) The HDS domains of GEA1 (749-1408) are GMH1binding sites (Dashed box in red) by which the SEC7 is stably recruited to the TGN and positively regulated via the HDS domains (Richardson et al., 2012). Source: Picture was taken from the reference (Chantalat et al., 2003).

\subsection{Pharmacological phenotypes of $u n c-50$ mutants in synaptic transmission}

The synaptic transmission in $C$. elegans can be phenotypically visualized by pharmacological assays by using levamisole, a cholinergic agonist of nAChRs, and aldicarb, an inhibitor of acetylcolinesterase (AChE) which is a hydrolytic enzyme of ACh in synaptic cleft (Fig. III.3A) (Mahoney et al., 2006). The mutants of unc-50 were initially identified on movement defects (Brenner, 1974) and shown to be extremely resistant on $1 \mathrm{mM}$ levamisole that, in wild type, causes muscle hyper-contraction, paralysis of worm body and death (Fig. III.3B). In order to clarify pharmacological phenotypes about the synaptic transmission of the unc-50 mutants ( $x 47$ and ok1847), unc29 (e1072) as a subunit of Lev-nAChRs (Fleming et al., 1997) and ric-3 (hm9) as a chaperone required for maturation of multiple nAChRs (Halevi et al., 2003) were used as positive-control mutant strains to compare resistant phenotypes. The unc-50 mutants were shown to be strong resistant on levamisole and aldicarb plates in both $100 \pm 0.00 \%$ at the time-points (Fig. III.4A and B), supporting that if a mutant is resistant to in both 
chemicals the gene mainly operates in the postsynaptic area, whereas if a mutant is resistant to aldicarb and sensitive to levamisole the gene is likely to operate in the presynaptic area (Mahoney et al., 2006). After four hours on aldicarb plates, $50 \%$ of unc50 mutants appeared to be kinker phenotypes (Fig. III.4C), indicating that the impaired neurotransmission in the synaptic cleft causes the mutant unc-50 to be severe kink and dead on the aldicarb plates (observed by the author). These results support that the UNC50 is mainly required for expression of certain molecules in postsynaptic membranes such as Lev-nAChRs, not in presynaptic areas (Eimer et al., 2007).

A

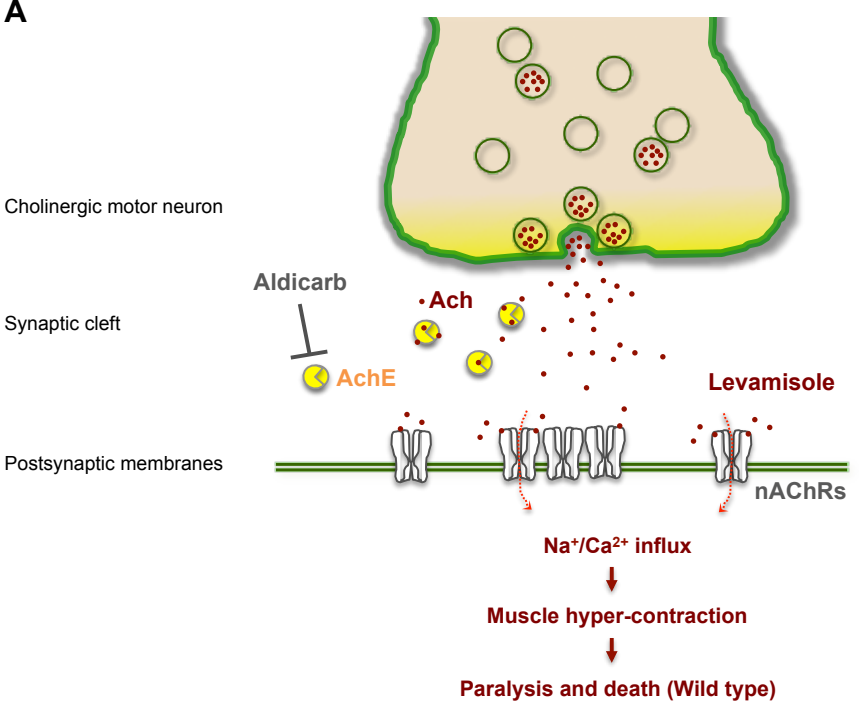

B

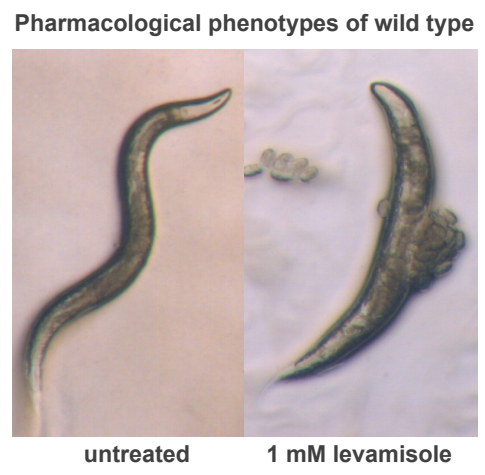

Figure III.3 Schematic representation of cholinergic neurotransmission and pharmacological phenotypes of wild type animals on $1 \mathrm{mM}$ levamisole. Synaptic transmission results in the activation of postsynaptic receptor-expressed membranes by neurotransmitters, which are released from presynaptic nerve terminal. C. elegans like mammals uses acetylcholine (ACh) which is released from cholinergic motor neurons to the postsynaptic membranes that allows the worm to make a sinusoidal movement by muscle contraction (Von Stetina et al., 2006). Levamisole, a cholinergic agonist of nAChRs, binds to one subtype of nAChRs (A), which leads to muscular hyper-contracted paralysis and death of wild type nematodes (B). Aldicarb is an inhibitor of acetylcolinesterase (AChE) that blocks the enzymatic hydrolysis of AChE to recycle choline back into presynaptic neurons in synaptic cleft (A). Therefore, impaired pre- and postsynaptic transmission in C. elegans can be easily distinguished by two chemical compounds, levamisole and aldicarb, that shown to be resistant and/or sensitive phenotypes. Source: Worm pictures of B were provided from Stefan Eimer. 
A

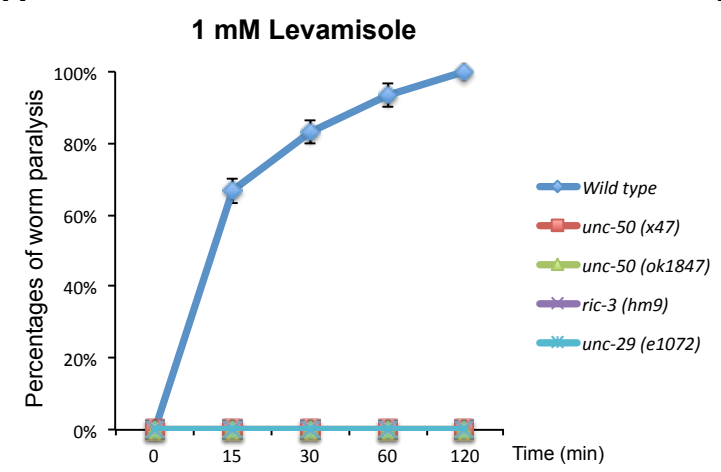

B

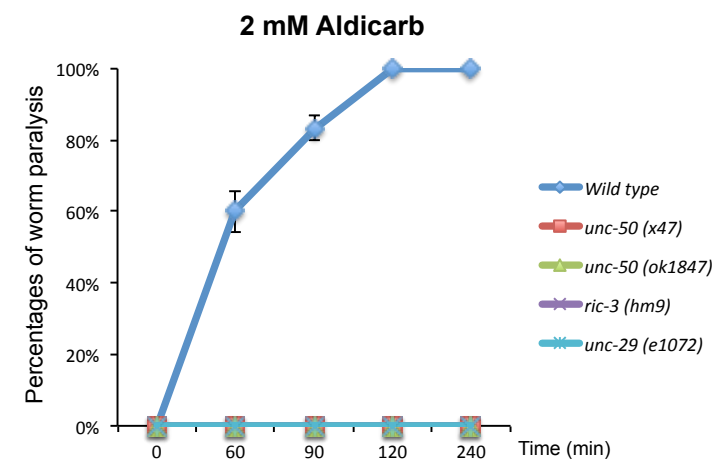

C

\begin{tabular}{|c|c|c|c|c|c|}
\hline Strains \Time & $5 \mathrm{~min}$ & $30-60 \mathrm{~min}$ & 4-hour & 1-day & Chemicals \\
\hline Wild type & Sine wave & Muscular contraction & Paralysis & Death & Lev \& Ald \\
\hline unc-50 mutants & Sine wave (uncoordinated) & - & - & No defect & Lev \\
\hline unc-50 mutants & Sine wave (uncoordinated) & - & $50 \%$ Kink & $100 \%$ Kink & Ald \\
\hline
\end{tabular}

Pharmacological phenotypes of the worms against Levamisole (Lev, $1 \mathrm{mM}$ ) and Aldicarb ( Ald, 2 mM)

Figure III.4 Pharmacological phenotypes of unc-50 mutants. UNC-50 is necessary for trafficking of levamisle-sensitive nAChRs (Lev-nAChRs) in the postsynaptic membranes. Pharmacological phenotypes of the unc-50 mutants were shown to be strong resistance, $100 \pm$ $0.00 \%$, in both $1 \mathrm{mM}$ levamisole and $2 \mathrm{mM}$ aldicarb plates (A and B). However, in the aldicarb assays, the approximately $50 \%$ worms of unc-50 mutants appeared to be kinker phenotypes after four hours and, in the following day (24 hours), the rest of worms (100\%) were shown to be severe uncoordinated phenotypes (Kink) with treatment on the $2 \mathrm{mM}$ aldicarb. In contrast, unc-50 mutants were not affected in the levamisole plates (C). Three independent experiments were performed on each strain. The number of worms was thirty per strain for analyzing.

\subsection{Analysis of synthetic lethal interactions of the trafficking factors in the TGN}

In the secretory pathway, dynamic sorting and trafficking of proteins take place at the trans-Golgi network (TGN) in which many critical factors related to the regulation of membrane trafficking are redundant in cellular processes (Altschuler et al., 1998; Baugh and Hunter, 2006; Ishizaki et al., 2008; Lode et al., 2002; Naor et al., 2005). In the case that redundant pathways exist in parallel at the TGN, functionally redundant genes in the pathway are able to compensate for the loss of gene. On the basis of the idea, unc-50 mutants have been examined by synthetic lethality analysis on the intracellular trafficking 
factors involved in the bidirectional anterograde and retrograde pathways in the interface of the TGN-endosomes (Table III.1A and 1B) where UNC-50 seems to mainly localize and function. Synthetic lethal phenotypes of the genetic interactions with unc-50 mutants obviously appeared in the mutant combinations with the retrograde trafficking factors (Table III.1B). This indicates that UNC-50 is likely to be functionally redundant with the factors Golgi associated retrograde protein complex (GARP), conserved oligomeric Golgi complex (COG), retromer complex and a small GTPase RAB-6, in the retrograde pathway. Synthetic interaction needs to be clarified precisely between the retrograde factors tested in the study.

To verify the synthetic lethality of the retrograde trafficking factors that function in parallel pathway, we examed the genetic interactions by synthetic lethal combinations between the retrograde factors: $\operatorname{cocg}^{-3}(\mathrm{kl} 181)$ as a subunit of COG complex, vps-35 (hu68) as a subunit of retromer complex, vps-53 (ok2864) as a subunit of GARP complex. They are required for sorting and recycling cargoes and tethering vesicles at the Golgi-endosomal interface (Bonifacino and Rojas, 2006; Conibear and Stevens, 2000; Seaman, 2005). As an upstream regulator for ARF-like 1 (ARL1), ADP-ribosylation factor (ARF)-related protein 1 (ARFRP1, arfrp1 (tm2779)) is needed for GRIP-domain recruitment to the TGN in both anterograde and retrograde pathway (Nishimoto-Morita et al., 2009; Panic et al., 2003; Setty et al., 2003; Shin et al., 2005; Zahn et al., 2006). Therefore, we tested these mutants to be lethal by crossing. Being seen in Table III.1C, the genetic interactions of ARFRP1 with VPS-53 (a subunit of GARP complex (arfprl; vps-53)) and VPS-35 (a subunit of retromer complex (arfprl; vps-35)) were shown to be severe defects in growth and egg-laying defects as well. However both are not embryonic lethality in the double mutant. On the other hand, the double mutant $\operatorname{cog} c-3$ (k181); vps35 (hu68) was genetically lethal (Table III.1C). As tested initially, $\operatorname{cog} c-3$ (k181); unc-50 (x47) and vps-35 (hu68); unc-50 (x47) resulted in embryonic lethal phenotypes, respectively (Table III.1B). This is consistent with the results that the double mutant in the GARP-COG complex (vps-52; $\operatorname{cog} c-1$ and $v p s-52 ; \operatorname{cog} c-3$ ) and GARP-retromer complex (vps-54; vps-35) were synthetic lethal phenotypes conducted by Ling Luo (Luo et al., 2011). In both organisms yeast and C. elegans, the genetic interactions were consistently shown to be lethal in combination with unc-50 mutants, but not in the 
anterograde trafficking factors, even the triple mutant syn-13 (tm2037); syn16 (tm1506); unc-50 (x47) was no defects (Table III.1B). Taken together, these results from the genetic interactions between the TGN genes, such as GARP, COG and Retromer complexes that are functionally redundant, support that UNC-50/GMH1 plays a functionally redundant role in the retrograde trafficking pathway in the TGN-endosomes.

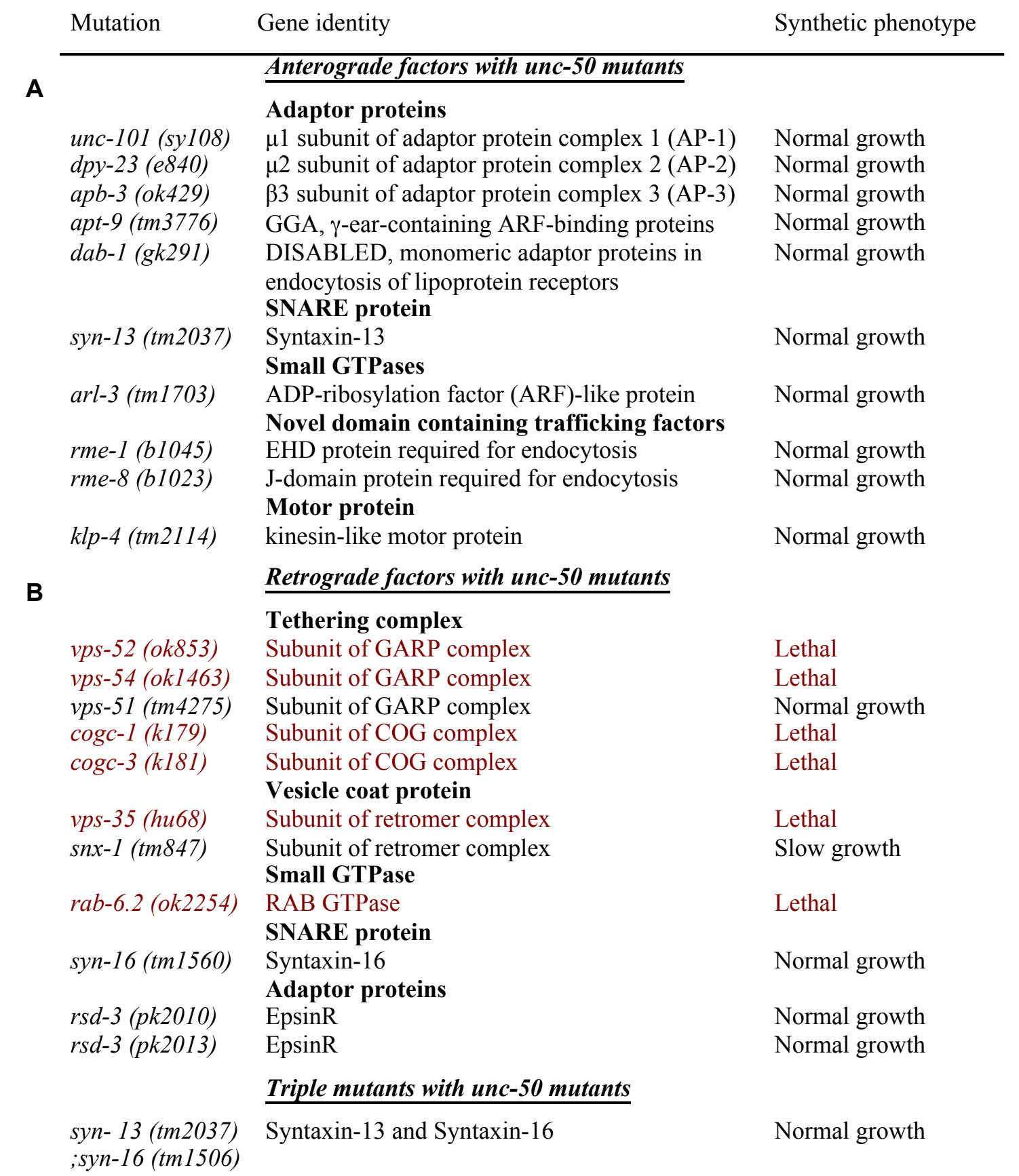




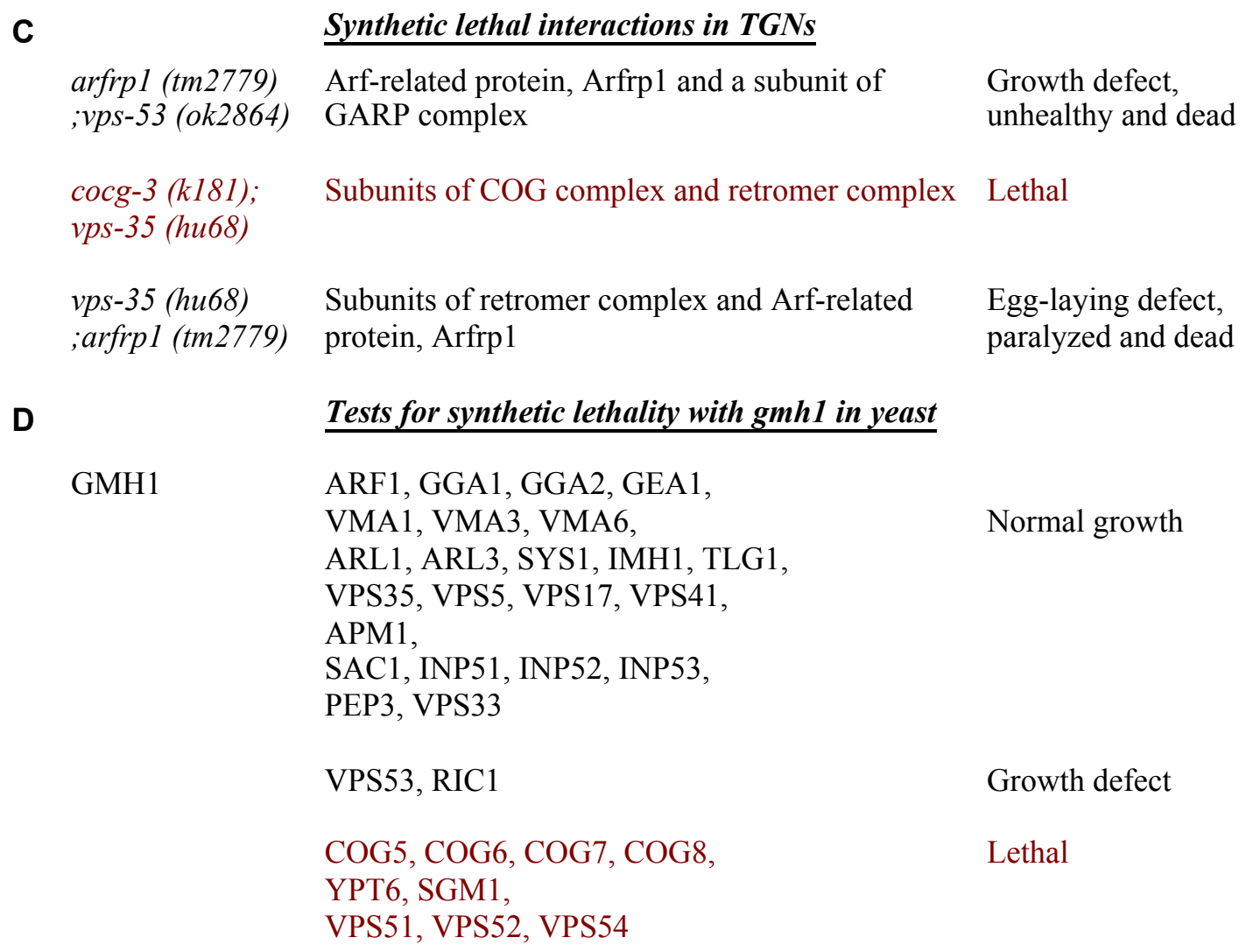

Abbreviations used in this table: Arf, ADP-ribosylation factor; GGA, Golgi localized, gamma-ear-containing Arf-binding protein; SNAREs, Soluble N-ethylmaleimidesensitive factor attachment protein receptors; TGN, trans-Golgi network; EHD, Eps15 homology domain as epidermal growth factor receptor substrates.

\section{Table III.1 Synthetic lethal phenotypes between the trafficking factors considered}

functionally redundant in the Golgi-endosomes. In the parallel pathway, synthetic lethality by double-mutants represents genetic interactions between two genes in the Golgi-endosomes. If there are only two pathways in function and both pathways are inhibited, it would show lethal phenotypes because there is no way to compensate the function by the other player. In the TGN, as a positive genetic interaction in parallel pathway, the genetic interactions of two genes $\operatorname{cog} c-3$; $v p s-35$, that act as a retrograde trafficking factor in COG and retromer complex, respectively, were shown to be lethal (C). This supports that genes in parallel pathway which are functionally redundant appear synthetic lethal phenotypes in the genetic combination. The tested genes shown to be lethal with unc-50/gmh1 mutants are all involved in the retrograde trafficking pathway in the endosomes-to-Golgi retrieval in two model organisms the yeast and C. elegans. Source: The 
experiments of synthetic lethal analysis were conducted by and Ling Luo (PhD dissertation, 2010) (A and B), the author (C) and Katrin Hartwich (D). 


\subsection{Aims of this study}

Highly conserved proteins required for intracellular trafficking play fundamentally important roles in eukaryotic cells. One of the conserved Golgi membrane proteins, UNC-50 is required for intracellular trafficking of Lev-nAChRs to the postsynaptic membrane at NMJs in C. elegans. The protein UNC-50 is highly conserved from yeast to humans, but the yeast $S$. cerevisiae and plant Arabidopsis do not have nAChRs. The yeast orthologous protein Gmh1p of the UNC-50 was not yet revealed about functional defects even it seems to be a critical transmembrane receptor as a binding partner of the ARFGEFs that regulates vesicle trafficking by activating ARF GTPases. To date, little is known about its conserved protein function of UNC-50/GMH1 as shown to be evolutionarily conserved in eukaryotic cells (Fig. II.7).

In case of the transport of Lev-nAChRs, due to the complexity of the multiple cargoes trafficking within intracellular interface, the selectivity of Lev-nAChRs by UNC-50 contributes to further insight into the regulation of the subtype-specific receptor trafficking since how UNC-50/GMH1 functions and its regulation mechanism remain unclear. To gain the insight of the specific-cargo trafficking by the ARF-GEFs membrane-mediated interaction partner UNC-50/GMH1, we focused on its function and intracellular phenotypes between the intracellular compartments where it works.

In Chapter I (Fig. III.5: STEP1-6), we show that UNC-50 localizes to the Golgiendosomal interface and functions as a recycling factor. unc-50/gmh1 mutants appear to be lethal or growth defects in combination with retrograde trafficking factors, GARP, $\mathrm{COG}$, retromer complex and RAB6, which can be considered as a redundant function of the protein required for retrieving cargoes back to the TGN. As a visual phenotype of unc-50 mutants shown in previous studies that the migration of posterior gonads was defected by regulating the secretion of MIG-17 through UNC-50-dependent pathway, the secretion defects of the signal sequence GFP fusion protein (ssGFP) from muscles were evident in C. elegans. Furthermore, inquiring aspects of intracellular organelles lacking UNC-50/GMH1 result in the targeting to be the lysosomal/vacuolar-bound phenotypes and enhanced recruitments as shown by using subcellular-organelle markers labeled with 
fluorescence from endosomes to lysosomes. In conclusion, we propose that UNC50/GMH1 as a membrane-mediated retrograde trafficking factor is required for recycling, secretion and trafficking of cargo proteins in the endosomal-lysosomal/vacuolar compartments.

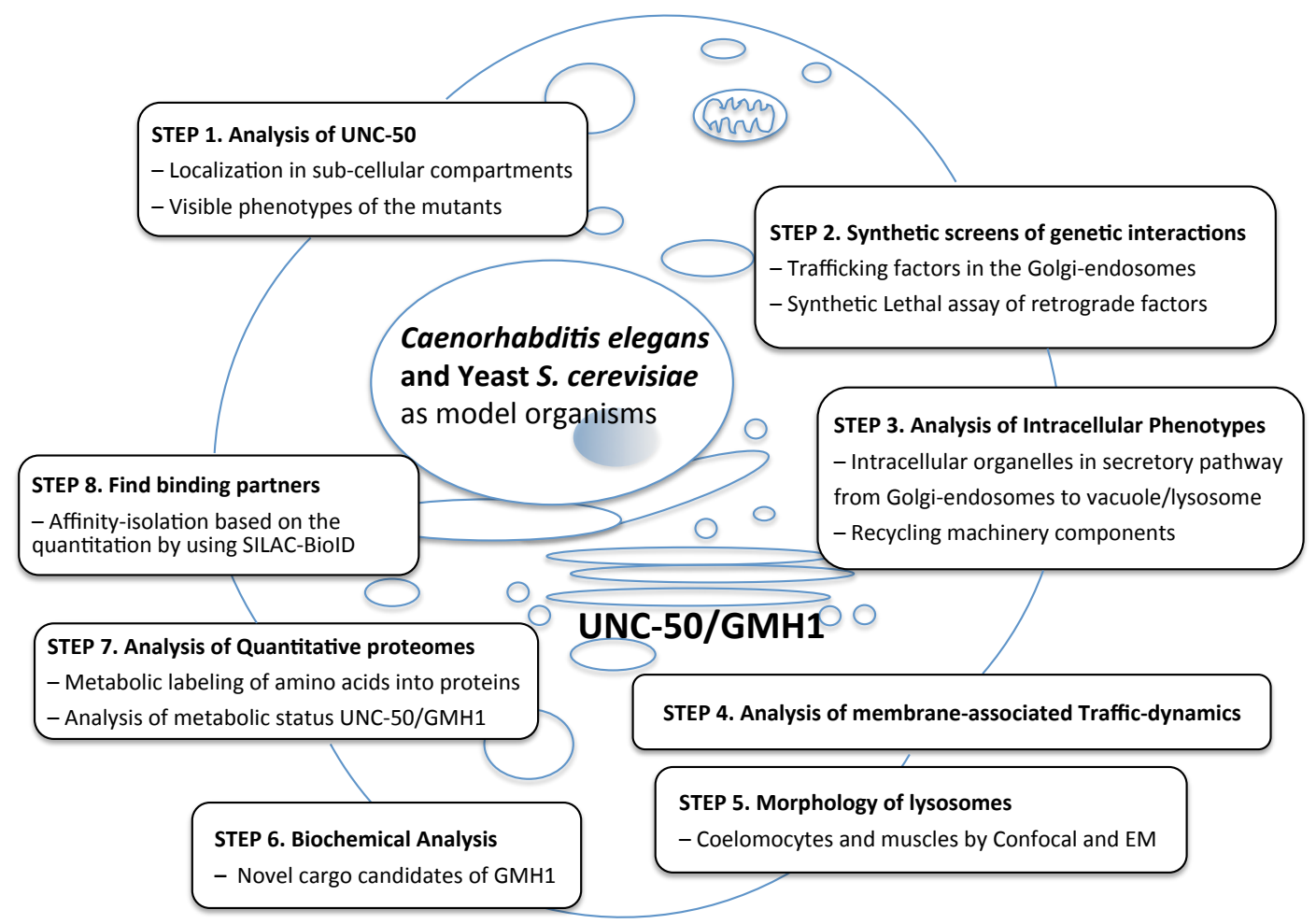

Figure III.5 Schematic diagrams of research aims for understanding the intracellular trafficking of UNC-50/GMH1-dependent. The project that has been done and doing by the author consists of eight parts divided into individual and collaborated works in two model organisms the nematode $C$. elegans and yeast $S$. cerevisiae. The STEP1-STEP6 belongs to the Chapter I: Analyzing the evolutionarily conserved function of UNC-50/GMH1 for membrane trafficking in yeast and C. elegans. The STEP1-STEP2 includes the data conducted by the author and Ling Luo. The STEP3, STEP4 and STEP6 were conducted by the author. The STEP5 was done by the author and Oliver Kretz. The STEP7-STEP8 belongs to the Chapter II: Proteomic/Metabolic analysis of the UNC-50 by stable-isotope labeling of amino acids in C. elegans which is ongoing experiments by the author and the collaborators in proteomics/metabolomics Core Facility of ZBSA, Uni-Freiburg (Carsten Jäger in metabolomics, Sebastian Wiese in proteomics and the group of Jörn Dengjel in proteomics). 


\section{RESULTS}

The evolutionarily conserved Golgi protein UNC-50/Gmhlp has been reported to be required for specific intracellular membrane trafficking events in yeast and C. elegans. However, the precise function of UNC-50/Gmh1p for intracellular membrane transport has yet to be determined (Chantalat et al., 2003; Eimer et al., 2007). In Chapter I, we focused on two model organisms yeast Saccharomyces cerevisiae and nematode Caenorhabditis elegans in order to identify the fundamentally conserved role of UNC50/Gmh1p by analyzing cellular phenotypes.

\subsection{UNC-50 colocalizes with APT-9 at the trans-Golgi networks}

In C. elegans, UNC-50 has been localized at the Golgi apparatus as reported previously by using a medial-Golgi marker alpha-Mannosidase II-GFP fusion protein (Eimer et al., 2007). In yeast, Gmhlp has been functionally linked to the early Golgi through its interaction with the ARF-GEF Gealp which is required for COPI dependent vesicular trafficking (Chantalat et al., 2003). However, UNC-50 does not colocalize with COPI coat components such as $\varepsilon$-COP in C. elegans as shown (Ling Luo). This suggests that UNC-50 might rather localize to late Golgi compartments or the TGN. To test this, we analyzed the localization of UNC-50 relative to APT-9 at the TGN.

APT-9 is the single C. elegans ortholog of GGA (Golgi-associated, gamma-adaptin ear homology and ARF-binding proteins) clathrin adaptor protein that is localized at the TGN. GGA adoptors facilitate ARF (ADP-ribosylation factor)-dependent clathrin recruitments (Boehm and Bonifacino, 2001; Boman et al., 2000; Dell'Angelica et al., 2000; Puertollano et al., 2001b; Robinson, 2004) and trafficking of sortilin and mannose 6-phosphate receptors (MPRs) from the TGN to the endosomal or lysosomal compartments (Ni and Morales, 2006; Nielsen et al., 2001; Puertollano et al., 2001a).

The N-terminus of APT-9 was fused to tagRFPt, a photostable red-fluorescent protein, and co-expressed with UNC-50::mYFP under the muscle specific promoter myo-3 (Table XII.3.3) in C. elegans. As shown in Figure IV.1, UNC-50::mYFP and tagRFPt::APT-9 
appeared to be highly colocalized at the TGN. This is consistent with the colocalization of GFP::GRIP domain at the TGN as previous experiments (Ling Luo) in which mRFP::UNC-50 was shown to be largely colocalized with an endosomal marker GFP::2xFYVE.

Taken together with our previous experiments, UNC-50 is likely to be localized at the TGN and/or Golgi-endosomal interface. At the TGN, UNC-50 guides the transport of the Lev-nAChRs to the plasma membrane and prevents their delivery to the lysosomes.

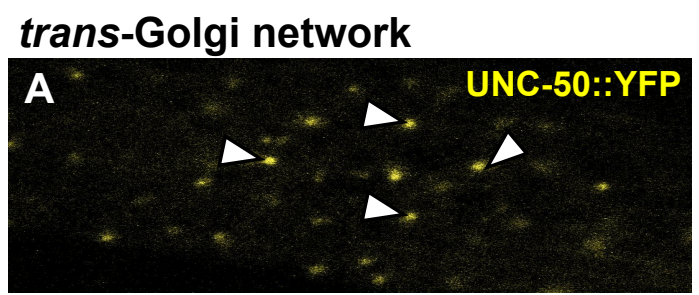

\section{Endosomes}
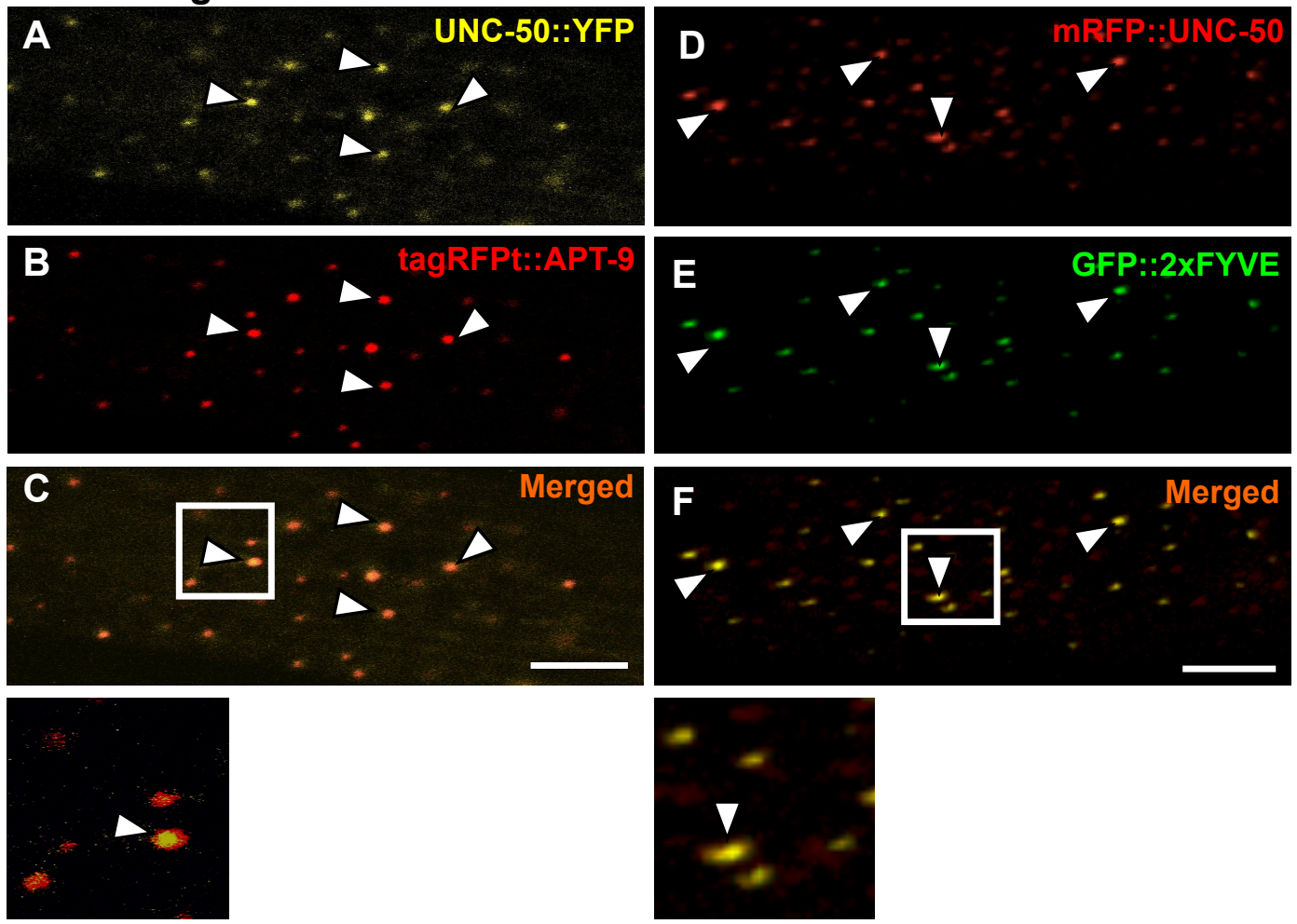

Figure IV.1 UNC-50 localizes to the trans-Golgi network and endosomal interface. Confocal images expressing the fluorescent-labeled organelle markers at the trans-Golgi network, tagRFPt::APT-9, and an early endosome, GFP::2xFYVE domain, are shown in the body wall muscles. UNC-50::YFP appears to be colocalized in both tagRFPt::APT-9 (A-C) and GFP::2xFYVE domain (D-F), respectively. The insets in the bottom of the figure were magnified from white box containing an arrow of each picture. Scale bars, $5 \mu \mathrm{m}$. Source: Above experiments were conducted by the author (A, B and C) and Ling Luo (D, E and F). 


\subsection{Phenotypes of cellular/functional expression of UNC-50/Gmh1p in eukaryotes}

The cellular phenotypes of UNC-50 reflect colocalization to the Golgi-endodomal interface. To find out the evolutionarily conserved function of UNC-50/Gmh1p, we further analyzed cellular phenotypes in yeast and C. elegans.

\subsubsection{Yeast growth phenotypes on non-fermentable and limited conditions}

The yeast ortholog of UNC-50, Gmh1p, has been shown to interact with Sec7-domain containing ARF-GEFs Gea1p and Gea2p (Gea1/2p) that are required for COPI-coated vesicular trafficking. This indicates that Gmh1p recruits Gea1/2p to the Golgi to locally activate Arf1 GTPases required for the generation and sorting of COPI-coated transport vesicles that mediate transport between the ER and Golgi compartment (Chantalat et al., 2003; Eimer et al., 2007). However, a more precise phenotypic analysis has not yet been done with gmhl mutants. In order to access the fundamental function of Gmhlp for $S$. erevisiae phenotypes, we analyzed the cellular growth phenotypes at different growth conditions. We found that yeast growth in the integrated gmhl-deleted strains $(\Delta g m h l)$ grew better than the wild type strain (BY4741) under iron-limited and starvation conditions (Data not shown).

In order to make sure the phenotype of Gmhlp, the screening for yeast growth phenotypes were designed with the iron chelators, Batho Phenantroline diSulfate (BPS) and Ferrozine (Cowart et al., 1993), non-fermentable condition plates and nutrient starved condition plates using Rapamycin, which is a component of mammalian target of rapamycin (mTOR) pathway inhibitor that involves in mitochondrial functions in energy and nutrient homeostasis (Cunningham et al., 2007) (All yeast media used in this study are listed in the Appendix). According to the growth phenotypes on iron-limited and starvation conditions, there was a strong possibility that Gmh1p would be involved in iron metabolisms and affected in nutrient regulations in yeast. To address these questions, the iron metabolism-related genes were assessed with gmh1. GEF1 known as yeast CLC proton-chloride exchangers in Saccharomyces cerevisiae is an evolutionarily conserved integral membrane protein. In the steady state it localizes in the late Golgi and prevacuolar compartments that depends on a soluble multicopper oxidase required for iron uptake, FET3, which accumulates the apo-FET3 in gefl deletion (Davis-Kaplan et 
al., 1998; Gaxiola et al., 1998; Greene et al., 1993; Schwappach et al., 1998; Yuan et al., 1997; Yuan et al., 1995). In particular, the GEF1 is known to be involved in cellular homeostasis for balancing the proton-chloride exchange in endosomal/lysosomal pathway for which it functions in the late Golgi and prevacuolar compartments (Gaxiola et al., 1999; Gaxiola et al., 1998; Scheel et al., 2005; Schwappach et al., 1998; Wachter and Schwappach, 2005). A vacuolar protease essential protein, PEP4, for protein degradation in the vacuole of yeast, was used to show the consistent hypothesis about the endolysosomal pathway. In the double-deletion of unc-50 with cup-5 (a lysosomal formation protein induced from endo-lysosomal organelles in C. elegans), the subunits of LevnAChRs were rescued as detectable levels by Western blots (Eimer et al., 2007). This means Lev-nAChRs target to the mis-routed way into the lysosome in the absence of UNC-50 in C. elegans.

In this study, the yeast strains used for growth phenotypes, wild type (BY4741), $\Delta g m h 1, \Delta g e f 1, \Delta g m h 1 ; \Delta g e f 1, \Delta p e p 4, \Delta f e t 3$ and $\Delta g m h 1 ; \Delta p e p 4$ (Appendix), were inoculated in yeast extract peptone-dextrose (YPAD) and/or synthetic complete (SC) liquid media (Appendix). For the assessment by spot dilutions on the solid media, yeast agar plates were prepared as follows: Synthetic Complete (SC) plates (D-glucose added), SC with BPS $(40 \mu \mathrm{M})$ plates, SC medium with Ferrozine $(3 \mathrm{mM})$ plates, yeast extract with 2\% glycerol-lactate (YP.Gly/Lac) plates and YP.Gly/Lac with Ferrozine (3, 1, 0.5 to $0.25 \mathrm{mM}$ ) plates. The yeast strains were grown in YPAD or SC media for overnight at $30^{\circ} \mathrm{C}$ and normalized to an optical density (OD) $600 \mathrm{~nm}\left(\mathrm{OD}_{600}\right)$ of 0.2 for six serial dilutions (until $2 \times 10^{-6}, \mathrm{OD}_{600}$ ) on each testing plate. Yeast growing phenotypes were no difference in either YPAD or SC. Instead, the growth phenotypes appeared to be depending on the fermentable (Fig. IV.2A-C) or non-fermentable carbon source plates (Fig. IV.2D-G). In gmh1 deletion strains, the growth phenotypes were consistently shown to be better than wild type on the non-fermentable conditions (Fig. IV.2A-C vs D). Thus, these results led us to thought that GMH1 might have effects on respiration and ironlimited growth involved in mitochondria functions.

As the results, the yeast growth phenotypes on the YP.Gly/Lac plates containing Ferrozine (3, 1, 0.5 and $0.25 \mathrm{mM}$ shown in Figure IV.2E) were shown to be similar in comparison with the YP.Gly/Lac plates without iron chelators (Fig. IV.2D-E). With this 
point in view, the growth phenotype of the double deletion strain $\Delta g m h 1 ; \Delta p e p 4$ was also similar phenotypes to a single mutant ( expression of proteins in yeast might be more tightly regulated in phenotype assays as shown in higher biological network systems (Eddy et al., 2010). In order to address the growth phenotypes of non-fermentable conditions (Fig. IV.2F), it was compared with the YP. Gly/Lac plates containing Rapamycin (4, 2, 1 and $0.5 \mathrm{nM}$ shown in Fig. IV.2G), considering that Gmhlp would be involved in nutrient metabolisms as shown to be resistant in starved conditions on YP.Gly/Lac plates. As shown in Figure IV.2G, the growth phenotypes of Gmh1p-depleted strains were not different from the phenotype of YP.Gly/Lac as a control (Fig. IV.2F).However, it has been shown to be much decreased in growth due to the non-fermentable-starved complex conditions. These results suggest that Gmh1p may not be closely or directly linked to involve iron-dependent pathways due to the indistinguishable phenotypes between non-fermentable and/or iron limited conditions by using YP. Gly/Lac plates. 


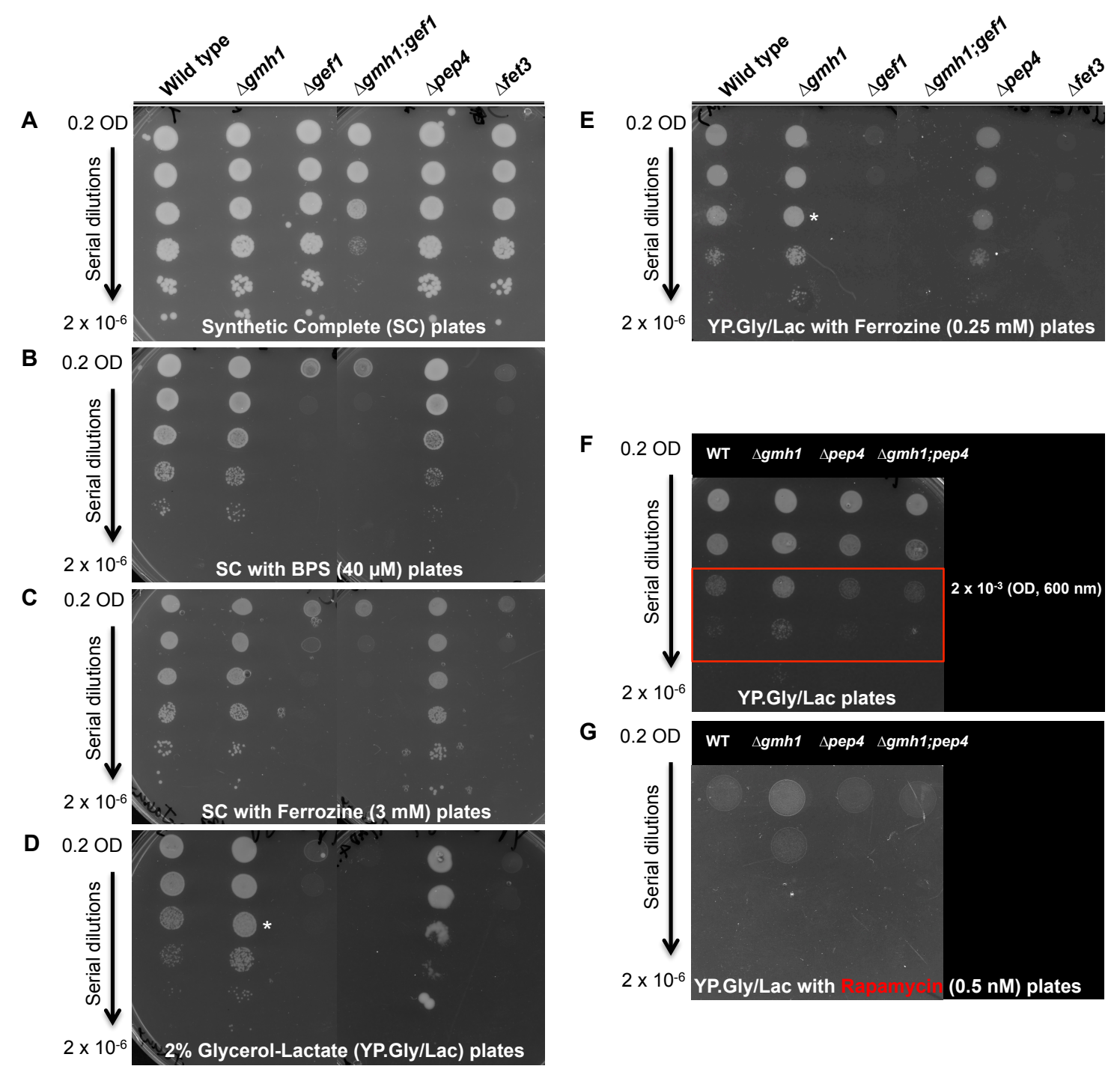

Figure IV.2 Yeast growth phenotypes on synthetic complete, non-fermentable and starvation conditions. The yeast cells used were grown in synthetic complete medium on fermentable carbon source plates (A, B and C) and non-fermentable carbon source plates (2\% Glycerol-Lactate yeast extract, YP.Gly/Lac) (D, E, F and G) for overnight at $30^{\circ} \mathrm{C}$ and normalized to an $\mathrm{OD}_{600}$ of 0.2 for six serial dilutions. Yeast growth phenotypes were shown to be better in $\Delta g m h 1$ strains on YP.Gly/Lac plates (D, E, F and G). Ferrozine (3, 1, 0.5 to $0.25 \mathrm{mM}$ ), Batho Phenantroline diSulfate (BPS) $(40 \mu \mathrm{M})$ and rapamycin (4 to $0.5 \mathrm{nM}$ ) were used to be ironlimited and starvation conditions. The serial diluted cells $\left(0.2\right.$ to $\left.2 \times 10^{-6}, \mathrm{OD}_{600}\right)$ were spotted and grown on the testing plates and incubated at $30^{\circ} \mathrm{C}$ for three days. Asterisks represent similar growth phenotypes in both 'with or without Ferrozine' (D and E) and the growth phenotypes of the red box $(\mathrm{F})$ are comparable to the rapamycin-containing plate $(\mathrm{G})$. 


\subsubsection{Analysis of intracellular organelle-specific markers in yeast $S$. cerevisiae}

The improved growth rates on a non-fermentable carbon source would give the possibility that gmh1 mutants contain a more active metabolism relying on mitochondrial respiration. Therefore, we analyzed the morphology and distribution of cellular compartments in gmh1 mutants of yeast cells.

In order to assess the effect of loss of Gmhlp function causes on intracellular trafficking between organelles, we used compartment specific markers such as ANP1RFP (Golgi marker), CHC1-RFP (endosomal marker), SNF7-RFP (prevacuolar marker) (Huh et al., 2003) and the dye FM 4-64 as a vacuolar marker in the gmhl-knockout background. gmh1 mutants and wild type yeast strains containing the plasmid pYESmtGFP by transformation as a mitochondria marker (Westermann and Neupert, 2000) were grown in $2 \%$ galactose to induce fluorescent protein expression.

To confirm the difference from the observation, three independent experiments at least were repeated by microscopy and Western blots in the same batch. As shown in Figure IV.3, CHC1-RFP (endosomes) and SNF7-RFP (prevacuoles) were shown to be strongly vacuolar-bound forms in gmh1 mutants (White arrows, Fig. IV.3). In contrast, ANP1RFP and pYESmt-GFP were not different when compared to the wild type (Fig. IV.3A). The vacuolar staining marker FM 4-64 showed that the vacuolar morphology in size as well as position were not affected in gmhl mutant cells. Expression levels of the markers as relative values were similar in amount and shown as non-significant, even though CHC1-RFP was destabilized in gmh1 deletion by Western blots (Fig. IV.3B). Interestingly, these results are consistent with the observation of intracellular markers by fluorescence in the Golgi-endolysosomal compartments of C. elegans (Fig. IV.4). These results indicate that $\mathrm{UNC}-50 / \mathrm{Gmh} 1 \mathrm{p}$ deletion induces enhanced recruitments of intracellular organelle markers related in trafficking to the endo-lysosomal pathway and $\mathrm{UNC}-50 / \mathrm{Gmhlp}$ is required for trafficking regulation in the intracellular compartments. 
A

\begin{tabular}{c|c|lll}
\multicolumn{1}{c}{ Mitochondria } & Golgi & Endosomes & Prevacuoles & Vacuole \\
\hline pYESmt-GFP & ANP1-mRFP & CHC1-mRFP & SNF7-mRFP & FM4-64 \\
\hline Wild type & & & & \\
\hline & & & & \\
\hline
\end{tabular}

B

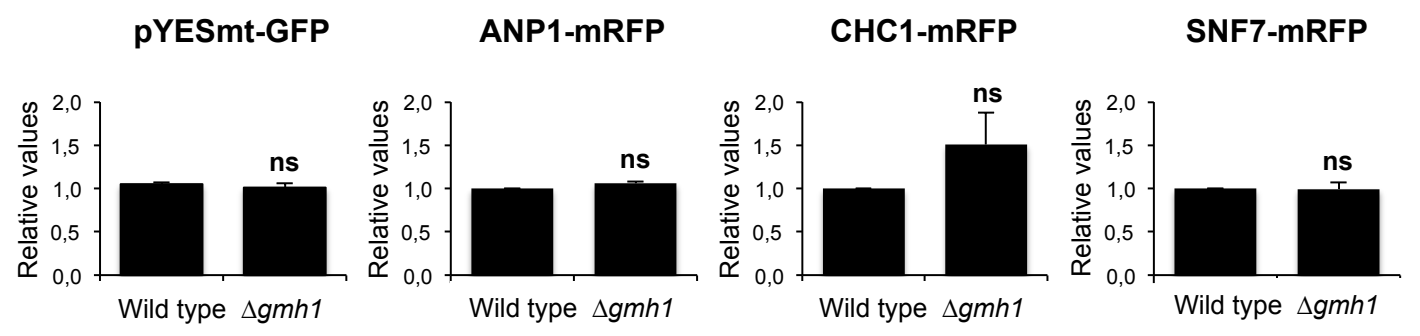

Figure IV.3 gmh1 mutants lead to enhanced trafficking to the vacuole in yeast Saccharomyces cerevisiae. Intracellular markers tagged by fluorescence were observed in different organelles as follows: pYESmt-GFP (mitochondria), Golgi (ANP1-RFP), endosomes (CHC1-RFP), prevacuoles (SNF7-RFP) and vacuoles (FM4-64) in wild type and gmh1 mutants $(\Delta g m h 1)$ (A). It has been shown that CHC1-RFP and SNF7-RFP in which the RFP was endogenously expressed in each strain and clearly showed strong intensity to vacuolar membranebound forms in gmh1 mutants. The total amount of the protein labeled by fluorescence was similar in Western blots (B). Three independent experiments were performed and error bars represent \pm s.e.m. ns, non-significant by Student's $t$ test. Scale bars, $5 \mu \mathrm{m}$.

\subsubsection{Analysis of intracellular organelle-specific markers in C. elegans}

To assess the trafficking interference by lacking UNC-50 in the intercellular compartments, the fluorescent-tagged markers characterized as the representative of each subcellular organelle in the Golgi-endolysosomal interface were analyzed in body wall muscles in C. elegans. The transgenic worms expressing each fluorescent-tagged gene 
under the control of the muscle specific myo-3 promoter in pPD115.62 were generated by microinjection of DNA constructs into the gonads (Mello et al., 1991) in wild type and unc-50 mutants ( $x 47$ and $o k 1847$ ). As shown in Figure IV.4, alpha-mannosidase II as a medial Golgi marker (Mans::tagRFP) (van den Elsen et al., 2001), 2xFYVE domains binding PI3P (GFP::2xFYVE) (Gillooly et al., 2000) and a small GTPase RAB-5 (RAB5::YFP) as early endosomal markers (Rink et al., 2005), a small GTPase RAB-7 as a late endosomal marker (mCherry::RAB-7) (Chavrier et al., 1990; Rink et al., 2005), APT-9 known as an ortholog of GGA (Golgi-localized, gamma-adaptin ear homology and ARFbinding protein) adaptor localized at TGN (tagRFPt::APT-9) (Robinson, 2004) and CTNS-1 as a lysosomal marker that encodes a homolog of human cystinosin as a cysteine transporter in C. elegans (Kalatzis et al., 2001; Town et al., 1998) were used in this study. It has been shown that enhanced intensity of the fluorescent-labeled markers in unc-50 mutants was observed and especially the small GTPase RAB-5::YFP and CTNS-1 appeared to be increased intensities and much clustered punctate structures compared to the wild type by confocal microscopy (Fig. IV.4A). Quantitative analysis of punctate size and the intensity was measured in the body wall muscles. Except for the markers of the Golgi and FYVE domain, the fluorescent-tagged markers RAB-5, RAB-7, APT-9 and CTNS-1 in TGN to endolysosomal compartments were shown to be significantly enhanced intensities in the $u n c-50$ depletion strains (Fig. IV.4B and C).

A cytosolic coatomer, COPI, highly conserved from yeast to mammals is an ARFdependent adaptor protein complex for coating vesicles involved in the retrograde trafficking in the Golgi-ER intermediate compartments and vesicle sorting from the lateendosomes to the lysosome (Cosson and Letourneur, 1997; Gabriely et al., 2007; Letourneur et al., 1994; Schekman and Orci, 1996; Serafini et al., 1991; Sohn et al., 1996). RAB-11, a small GTPase, mediates mainly recycling endosomes in endocytosis and exocytosis (Grant and Donaldson, 2009; Stenmark, 2009; Takahashi et al., 2012; van Ijzendoorn, 2006). One subunit of COPI consisting of seven subunits $(\alpha, \beta, \beta, \gamma, \delta$, $\varepsilon$ and $\zeta$ ) mCherry- $\varepsilon$ COP and tagRFPt-RAB-11 were monitored in the body wall muscles of young adults in wild type and unc-50 mutants ( $x 47$ and $o k 1847)$. As a coated protein organelle marker, mCherry- $\varepsilon$ COP was shown to be strong intensity of the fluorescence in 
unc-50 mutants (Fig. IV.5A), whereas the recycling endosomal marker, tagRFPt-RAB11, was not significantly different from the wild type (Fig. IV.5B).

These results suggest that UNC-50 is likely to be involved in COPI mediated-ARFdependent and RAB small GTPase-dependent vesicle trafficking in the Golgiendolysosomal interface. Therefore, in the absence of UNC-50, cargoes such as the receptor subunits of Lev-nAChRs seem to be re-routed or mis-localized to the lysosome under the coated vesicles with the consecutive ARF/RAB small-GTPases membranemediated trafficking by UNC-50-dependent-pathway.

A
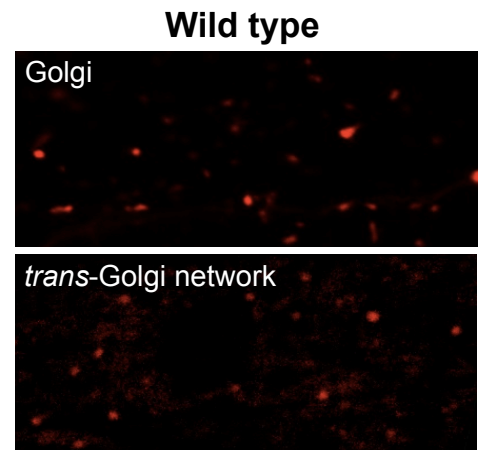

Early endosomes
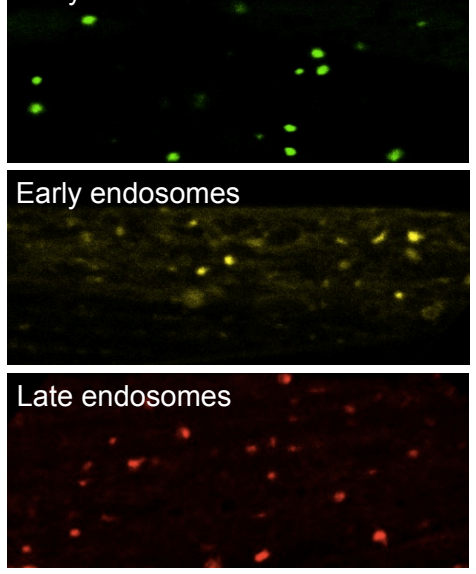

Lysosomes unc-50 (x47)
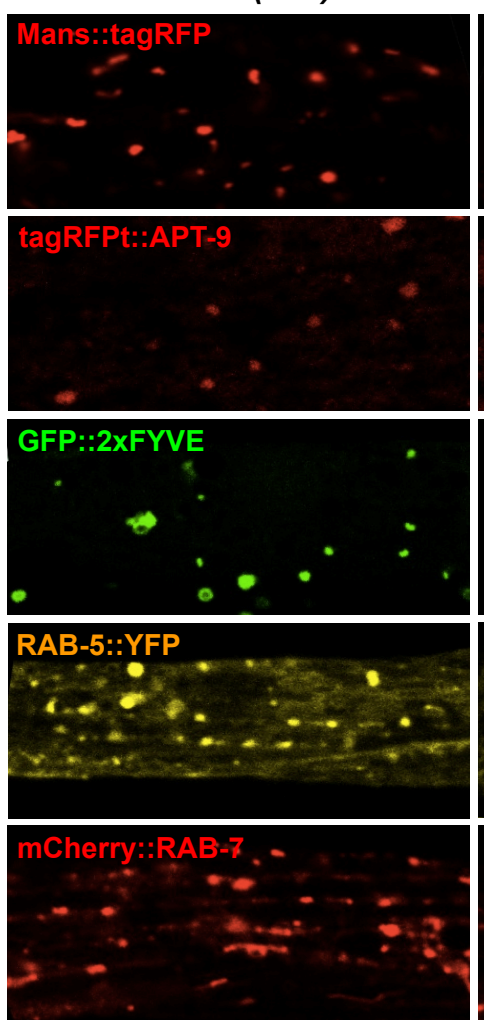

CTNS-1:YFP

CT
-

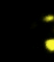


B

Mans::tagRFP

GFP::2xFYVE
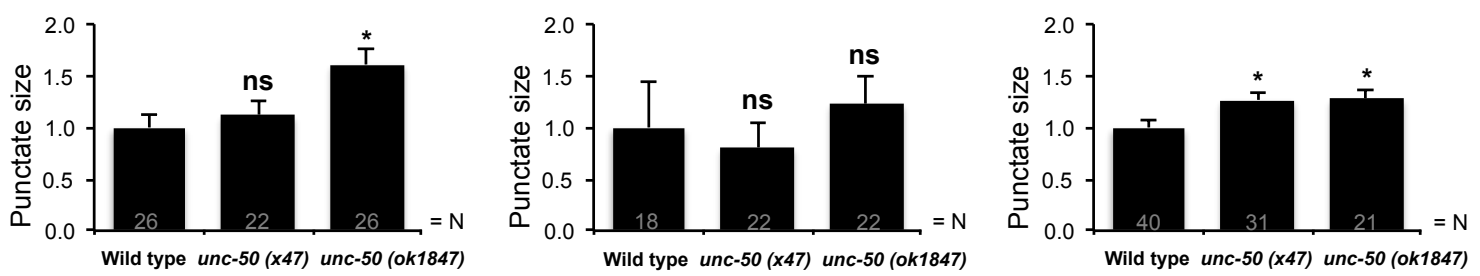

tagRFPt::APT-9
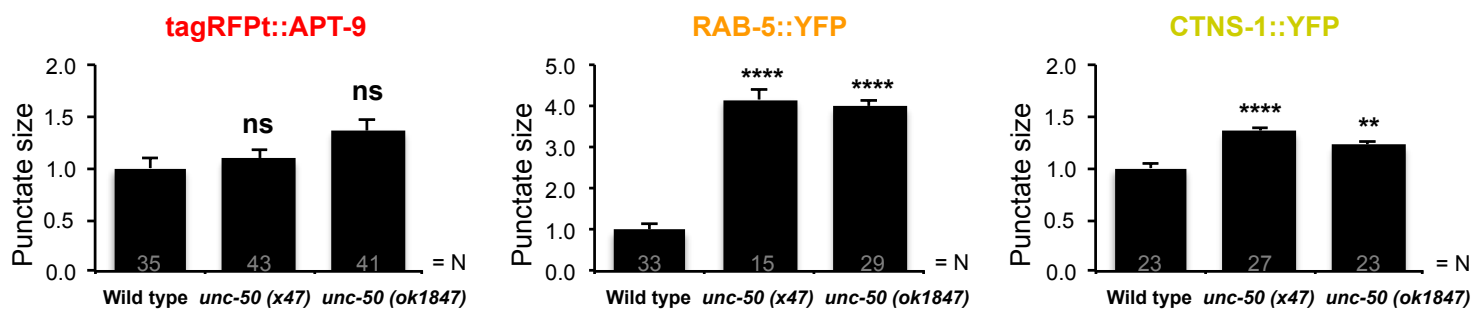

\section{C}

Mans::tagRFP

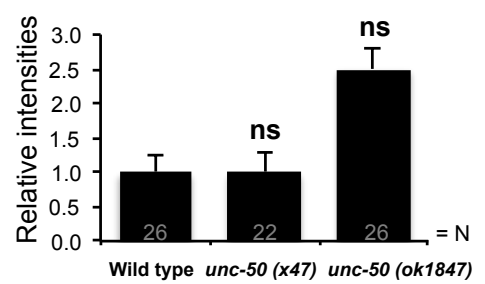

tagRFPt::APT-9
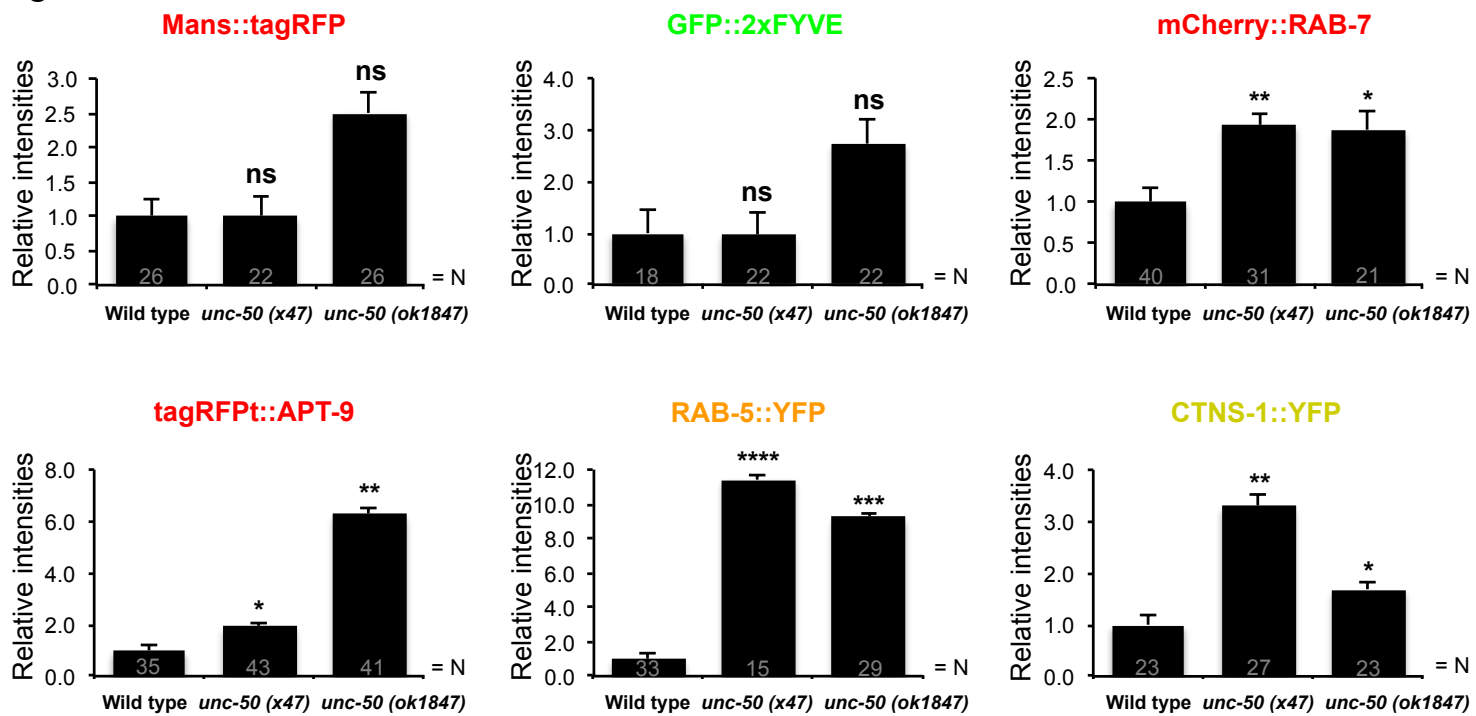

Figure IV.4 unc-50 mutants show enhanced intensity of the fluorescent-tagged organelle specific markers in the Golgi-endolysosomal compartments in $C$. elegans. Confocal images expressing the fluorescent-labeled markers in organelles are shown in wild type and unc-50 mutants ( $x 47$ and ok1847). It has been observed that the Golgi-endolysosomal interface in which Golgi ( $\alpha$-mannosidase II labeled-tagRFP), early endosomes (GFP-tagged 2xFYVE and RAB-5 tagged-YFP), late endosomes (mCherry-tagged RAB-7), a trans-Golgi network (tagRFPt-tagged APT-9) and lysosomes (CTNS-1 tagged by YFP) were monitored in the body wall muscle cells of young adults. Scare bars, $5 \mu \mathrm{m}$ (A). Analysis of the punctate size of the corresponding fluorescent-labeled organelle markers (B) and the relative intensity were assessed in the endolysosomal pathway (C). At least three independent experiments were performed on each 
strain. Total number of worms analyzed is presented in the graph bars. Error bars represent the standard error of the mean ( \pm s.e.m). Asterisks indicate significant between wild type and unc-50 mutants as follows: respective * $\mathrm{P} \leq 0.05 ; * *, \mathrm{P} \leq 0.01$; ***, $\mathrm{P} \leq 0.001$; ****, $\mathrm{P} \leq 0.0001$; $\mathrm{ns}$, non-significant by Student's $t$ test.

A

COPI-coat complex Wild type
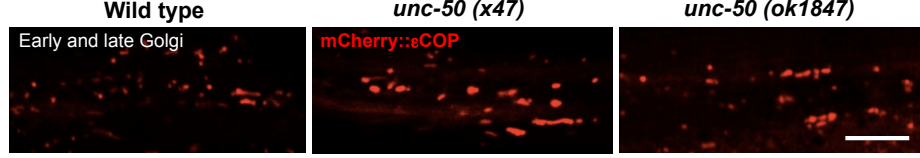

B

RAB-11
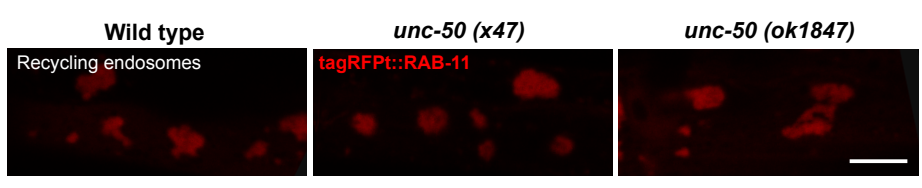

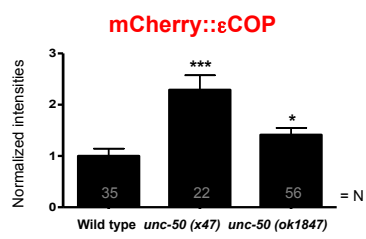

tagRFPt::RAB-11

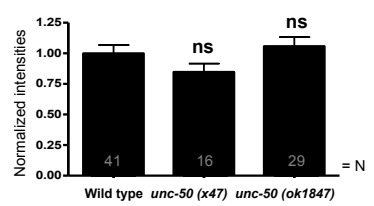

Figure IV.5 A coat protein subunit of COPI, mCherry- $\varepsilon$ COP, show enhanced intensity in unc-50 mutants in the body wall muscles, but not in recycling endosomes, tagRFPt-RAB-11. Confocal images expressing the fluorescent-labeled markers in a coated organelle marker, mCherry-eCOP (A) and a recycling endosomal marker, tagRFPt-RAB-11 (B), in the body wall muscles of young adults in wild type and unc-50 mutants ( $x 47$ and ok1847). Three independent experiments were performed on each strain. Total number of worms analyzed is presented in the graph bars. Error bars represent the standard error of the mean ( \pm s.e.m). Asterisks indicate significant between wild type and unc-50 mutants as follows: respective *, $\mathrm{P} \leq 0.05 ; * *, \mathrm{P} \leq 0.01$; $* * *, \mathrm{P} \leq 0.001 ; * * * *, \mathrm{P} \leq 0.0001 ; \mathrm{ns}$, non-significant by Student's $t$ test. Scare bars, $5 \mu \mathrm{m}$

\subsection{Morphological analysis of coelomocytes and muscles in unc-50 mutants}

\subsection{1 unc-50 mutants show relatively enlarged lysosomes in coelomocytes}

In $u n c-50$ mutants, the positive vesicles of the lysosome were observed and compared in size between wild type and unc-50 (x47) by using an integrated transgenic worm strain [pwIs50] expressing a lysosomal marker tagged by GFP (LMP-1::GFP) in coelomocytes. Given that the trafficking aspects of the enhanced recruitments were observed by fluorescent-tagged subcellular markers in the Golgi-endolysosomal interface (Fig. 
IV.4A), we compared the lysosomal size in wild type with unc-50 mutants. As can be seen in Figure IV.6A, LMP-1::GFP was shown to be significantly enlarged GFP-positive vesicles in size in unc-50 (x47) mutants when compared to wild type. The ratio of the vesicles expressing GFP was either analyzed in size, in which they were divided into two groups: less than $1 \mu \mathrm{m}$ and more than 1 to $3 \mu \mathrm{m}$ (Fig. IV.6B). Most of the GFP-positive vesicles were shown to be less than $1 \mu \mathrm{m}$ in $70 \% \pm 0.16$ of the wild type and no GFPpositive vesicles as more than $3 \mu \mathrm{m}$ were found in the wild type unlike unc-50 mutants. According to recent studies related to the lysosomal morphology in size, the absence of GARP complex subunits vps-52, vps-53, vps-52 and vps-51, which are known to be required in recycling and tethering vesicles with SNARE complexes through a retrograde trafficking manner in the Golgi-endosomal interface, causes enlarged lysosomes (Luo et al., 2011). The LMP-1::GFP in unc-50 (x47) was much similar to the vps-51 (tm4275) rather than other subunits in the size of the positive vesicles. This suggests that, with the genetic interaction experiments shown as lethal phenotypes between unc-50/gmh1 and retrograde trafficking factors such as COG, GARP, retromer and RAB-6 in the two model systems (Table III.1), this funding is consistent with the expectation that UNC-50 and GARP complex have redundant functions in the Golgi-endosomal interface. 
A

Lysosomes in coelomocytes
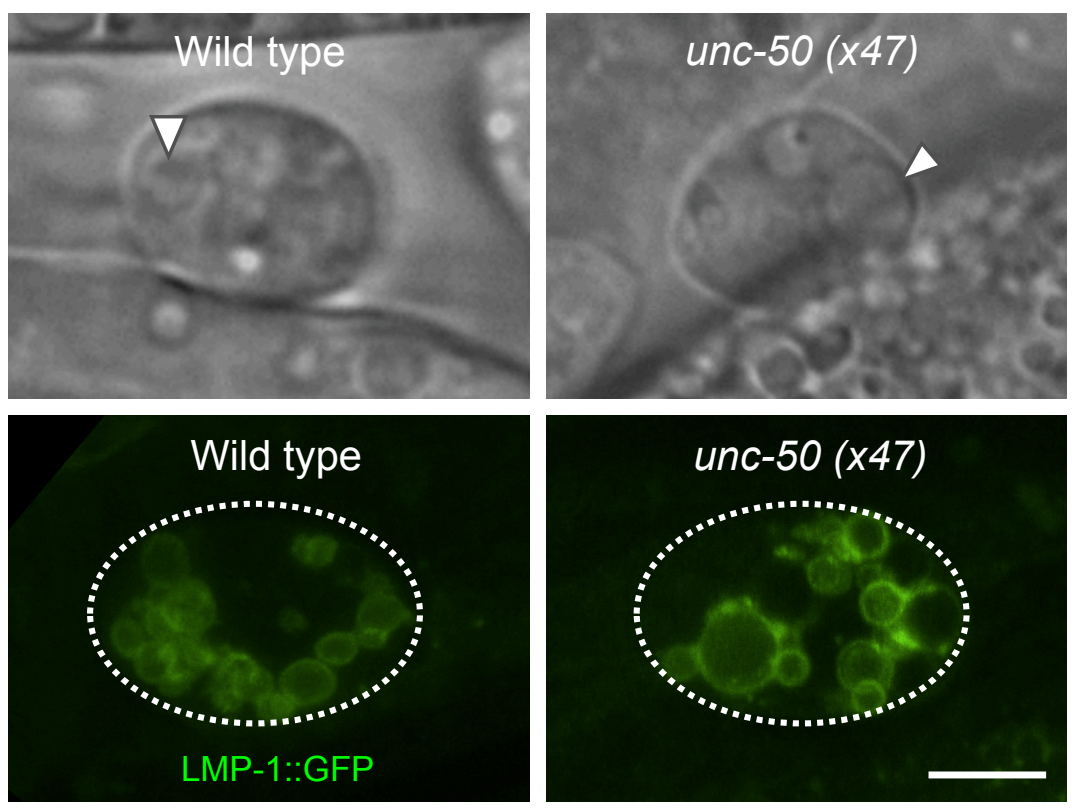

B

Ratios of GFP vesicles by size

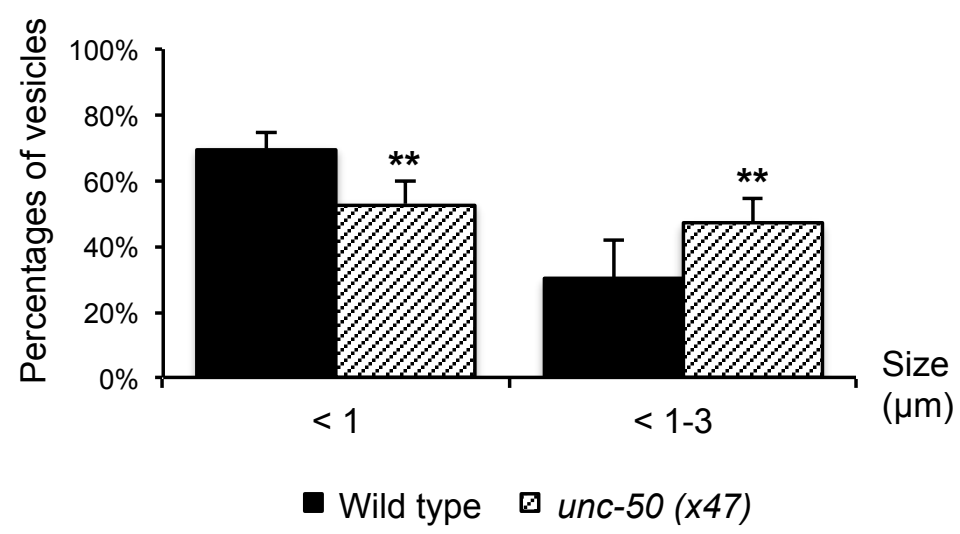

Figure IV.6 unc-50 mutants show relatively enlarged lysosomes in coelomocytes. The lysosomal marker LMP-1 expressing GFP (LMP-1::GFP) appears to be relatively enlarged positive vesicles in unc-50 (x47) mutants by phase contrast and confocal microscopy. Scale bars, $5 \mu \mathrm{m}$ (A). The positive vesicles of LMP-1::GFP were divided into two groups by size as follows: the positive vesicle $<1 \mu \mathrm{m}$ and $1-3 \mu \mathrm{m}$ or more (B). Total number of analyzed animals is 23 of wild type (the average of positive vesicles: $10.3 \pm 2.1$ ) and 21 of unc-50 mutants (the average of positive vesicles: $10.5 \pm 2.6$ ), respectively. Each group was shown by percentages of the GFP 
positive vesicles. Error bars represent \pm s.e.m and the significant **, $\mathrm{P} \leq 0.01$ values are analyzed by Student $t$-test.

\subsubsection{Morphological analysis of the body wall muscles by HPF-EM}

Typical somatic muscle cells in C. elegans can be divided into three parts: contractile, non-contractile and muscle arms. The contractile filament lattice contains sarcomeres, a non-contractile body is called as a muscle belly including mitochondria and organelles in the cytoplasm (Bird and Bird, 1991) (www.wormatlas.org). For the morphological analysis of the body wall muscles, young adults of unc-50 (x47) mutants were compared to wild type animals, which were analyzed by using high-pressure freeze electron microscopy (HPF-EM). In the longitudinal sections, the unusual vesicles (lipid-like) in various sizes near mitochondria in the muscle belly were largely observed in unc-50 mutants unlike wild type (Fig. VI.7), suggesting UNC-50 may affect in lipid metabolisms of the worm.

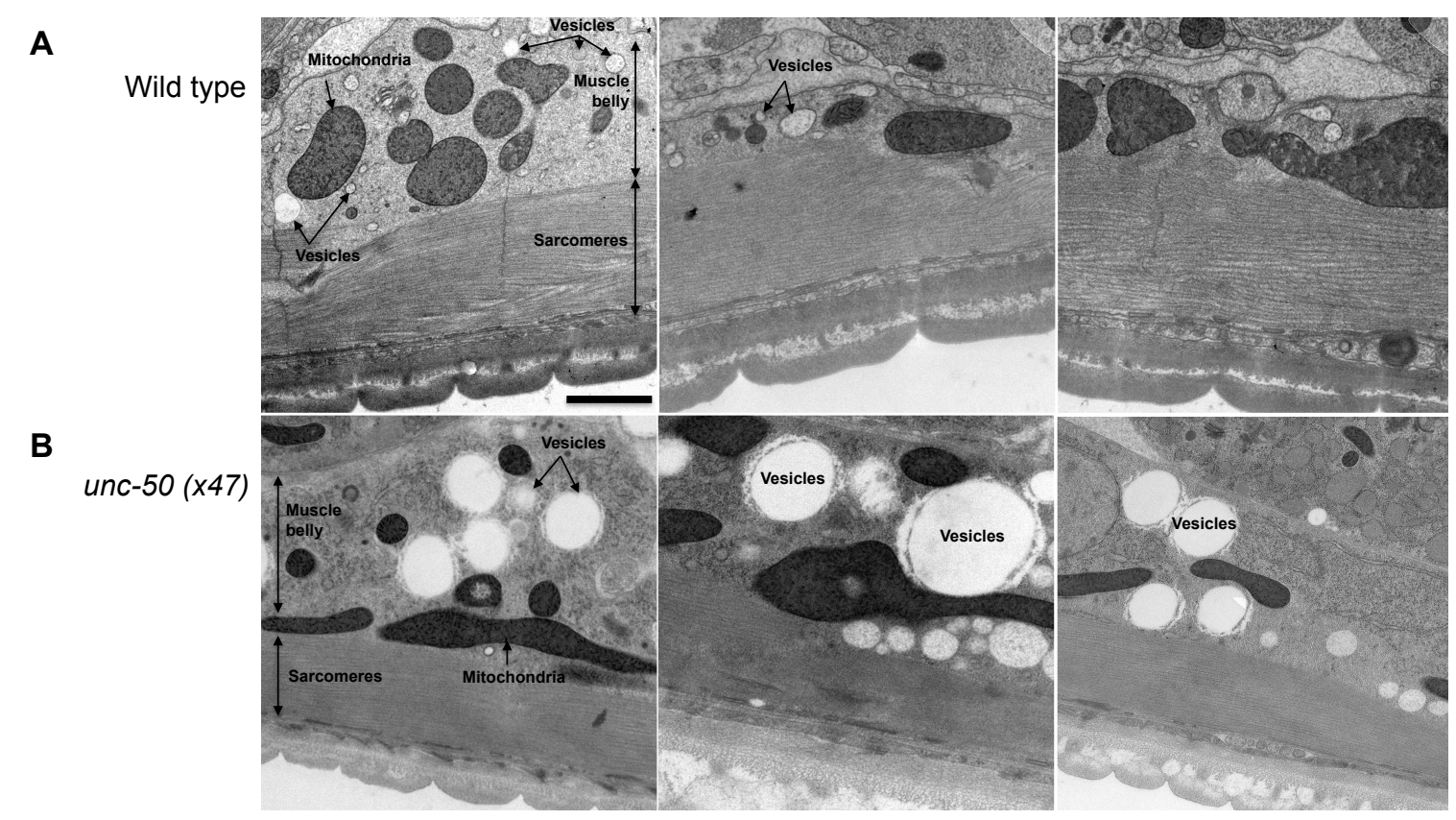

Figure IV.7 HPF-EM analysis of the organelle morphology in C. elegans muscles. The longitudinal section views are shown in wild type and unc-50 mutants. In the muscle belly as a non-contratile body, mitochondria and organelles are commonly observed in both strains by HPFEM. Scale bars, $1 \mu \mathrm{m}$. Source: The HPF-EM was conducted by Oliver Kretz and the pictures were kindly provided from him. 


\subsection{Intracellular traffic-dynamics to the vacuolar/lysosomal compartments}

Through the synthetic lethal assays, it has been revealed that UNC-50 was shown to be involved in the retrograde transport such as GARP, COG, retromer complexes and RAB6 at the Golgi-endosomal interface. Interestingly, unc-50/gmh1 mutants had no defects and shown to be normal growth with the absence of the factors involved in the anterograde transport factors or endocytosis by the synthetic lethal analysis (Table III. 1A). These results indicate that UNC-50/GMH1 may be not required for the cellular processes in the anterograde transport as well as endocytosis, but that needs to be verified, in the intracellular compartment.

\subsubsection{Bulk-fluid phase endocytosis functions in unc-50 mutants}

In order to know the capability of endocytosis in the depleted status of UNC-50, the coelomocyte that is a macrophage-like scavenger cell in C. elegans were observed for checking endocytosis in the bulk-fluid phase with Texas Red labeled BSA (TR-BSA, $1 \mathrm{mg} / \mathrm{ml}$ ). The coelomocyte is known as highly active in membrane transport by bulk endocytosis from pseudocoelomic body fluids into the cell in C. elegans (Fares and Greenwald, 2001). For this test, the TR-BSA was injected into the body cavity of the pharyngeal region of the transgenic worm strains, bIs34 [prme-8::rme-8::GFP] expressing GFP as endocytic markers, and observed uptake in coelomocytes as time series (10, 30 and 50 minutes). After injection of TR-BSA, worms were mounted on icechilled $2 \%$ agarose pads to observe the endocytic dynamics at the indicated time points in the same conditions by confocal microscopy (Figure IV.8). The endocytic aspects were shown to be similar in both wild type and unc-50 (x47) mutants. After 10 minutes of the injection, the positive structures were already shown in late endosomes by endocytosis. After 30 minutes, TR-BSA was shown in the inside and the outside of RME-8::GFP vesicles in coelomocytes. At 50 minutes, most of TR-BSA appeared in later structures. These results suggest that endocytosis seems not to be affected by unc-50 mutants, in particular, in the bulk-fluid phase and these results are consistent with the fact that UNC50 is not required for the trafficking pathway involved in the general endocytosis. 


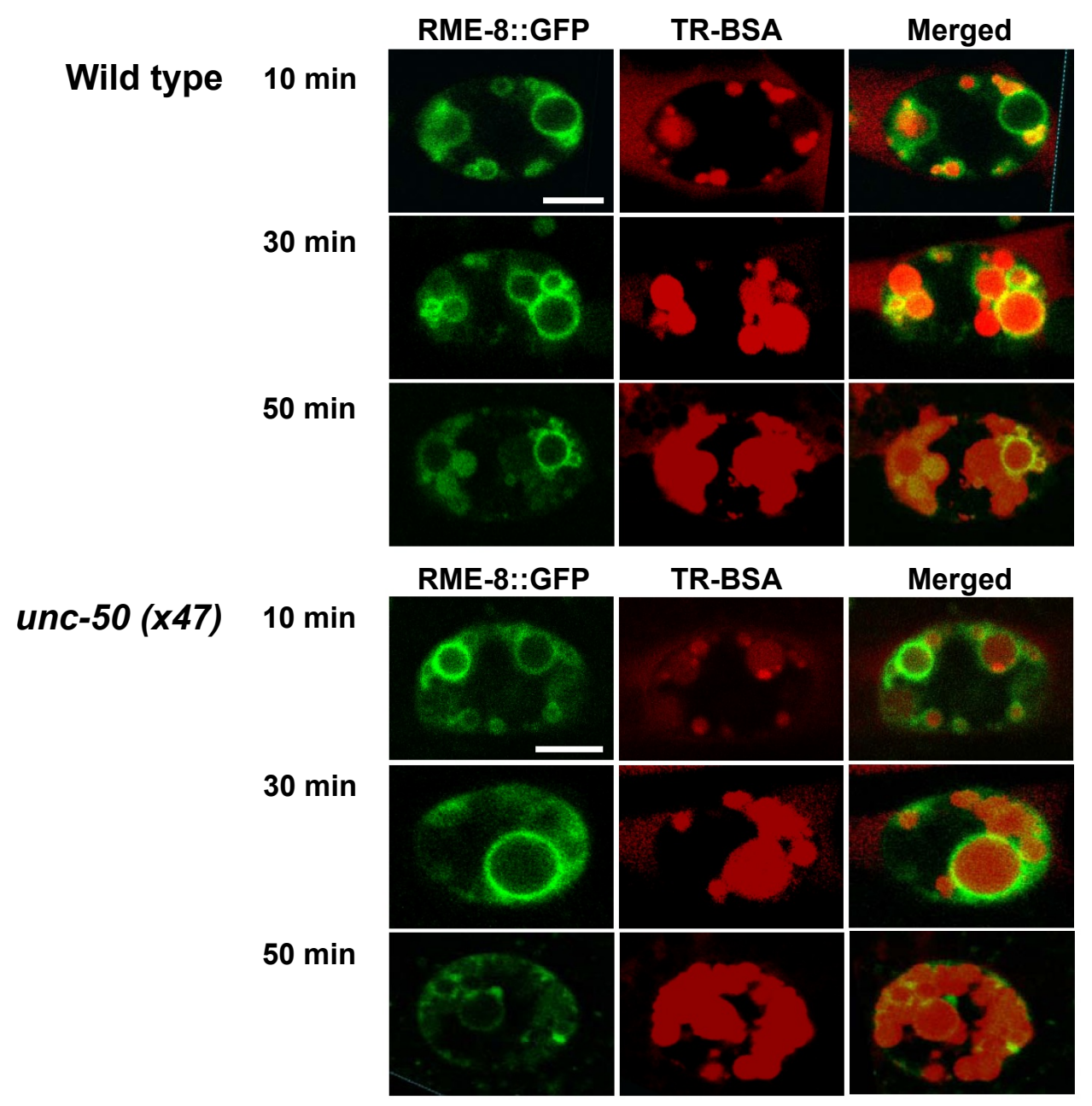

Figure IV.8 Coelomocytes show normal dynamics of endocytosis by bulk-fluid phase in unc50 (x47) mutants. Confocal images of coelomocytes expressing late endosomal marker RME$8::$ GFP after injection of TR-BSA $(1 \mathrm{mg} / \mathrm{ml})$ at the indicated time points $(10,30$ and 50 minutes $)$. Scale bars, $5 \mu \mathrm{m}$.

\subsubsection{FM4-46 dyes reach fast to the vacuolar membranes in gmh1 mutants}

To characterize the endocytic aspects of the intracellular trafficking in gmhl mutants, we examined membrane dynamics of endocytosis by tracking FM 4-64, a lipophilic styryl dye as a vital stain of yeast cells (Fig. IV.9). The internalized trafficking way of FM 4-64 from plasma membranes to endosomes and finally vacuolar membranes in the cytoplasm is likely to be affected by temperature, time, energy dependent and transport factors while they are in the process of being internalized by endocytosis (Vida and Emr, 1995). 
UNC-50 is known as involved in sorting and trafficking of Lev-nAChRs and its depletion was shown that the receptor's subunits were degraded in lysosome in $C$. elegans (Eimer et al., 2007). We found in this study that gmh1/unc-50 mutants lead to the enhanced trafficking to the vacuole/lysosome through the recruitments of interacellular organelle markers of the Golgi-endolysomal compartments in both yeast $S$. cerevisiea and C. elegans (Fig. IV.3 and Fig. IV.4). This indicates that gmhl depletion would evoke transportation mobility of certain molecules to the vacuolar membranes by its endocytosis of FM 4-64. In order to determine that how gmh1 deletion affects in endocytosis of yeast cells under steady state conditions, FM 4-64 was used for visualizing membrane dynamics and observed by chasing the red-fluorescent labeling dyes of the membrane as the indicated time points (10, 20, 30 and 50 minutes) (Fig. IV.9C and 10A). Before checking the mobility of the dye, yeast cells were treated for 30 minutes at $0^{\circ} \mathrm{C}$ in FM 4-64 as a final conc. $40 \mu \mathrm{M}$, and then the cells with FM 4-64 were analyzed by microscopy at each time point (Fig. IV.10). In gmhl mutants, dynamics of the endocytosis was observed to be rapidly reached to the vacuolar membrane compared to the wild type at early time point within 20 minutes. To analyze the difference from the control, the images were divided into three patterns (Fig. IV.10B): Ring (plasma membranes), Dots (small punctate structures in endosome-like membranes) and Vacuoles (vacuolar membranes) at the indicated time points, and by which the staining cells were measured as four time-intervals at 10 ( 1 to 15 minutes), 20 (15 to 25 minutes), 30 (30 to 40 minutes) and 50 (50 to 60 minutes) that were repeated by four independent experiments (Fig. IV.10B). After treatment of the dye, the ring-staining pattern of the styryl dye was shown to appear at the outer membrane and taken up to rapidly endosomelike membranes shown as small punctate structures in the cytoplasm. It was then eventually attached to the vacuolar membrane in both strains. Interestingly, at early timepoints (10 and 20), vacuolar-staining patterns came out in gmhl mutants first and its significance was shown to appear continuously in the time point at 20 (15 to $25 \mathrm{~min}$ ) (Fig. IV.10B). After 30 minutes, they were shown to be not significant difference anymore and most of them were observed on the vacuolar membranes in both after 50 minutes. Thus, these data suggest that the endocytosis not only functions normal in gmhl 
mutants but also its dynamics is affected and rather faster in the trafficking to the vacuolar membrane in the absence of GMH1.

A

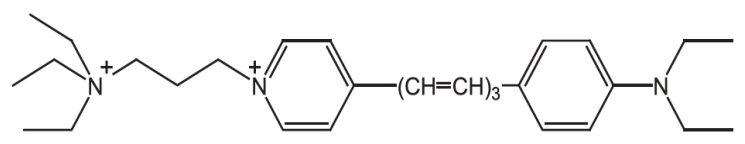

FM 4-64
$2 \mathrm{Br}^{-}$
B

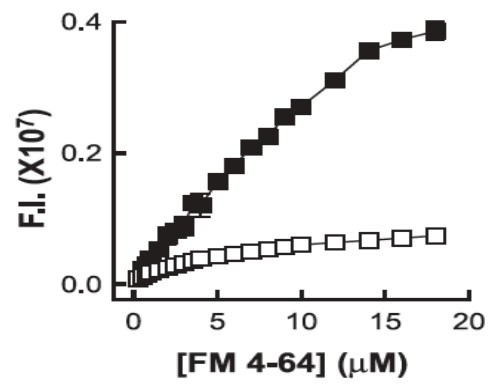

C

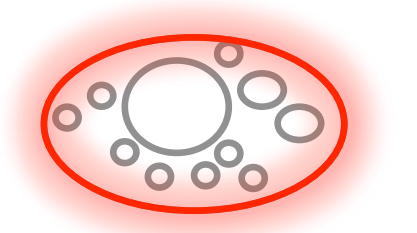

$1 \mathrm{~min}$

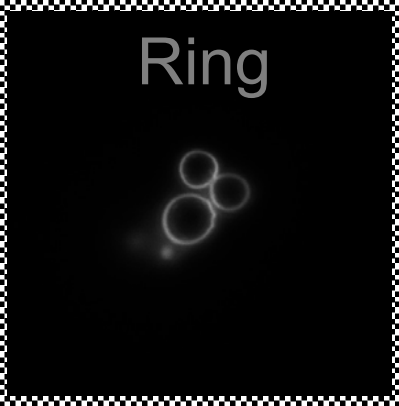

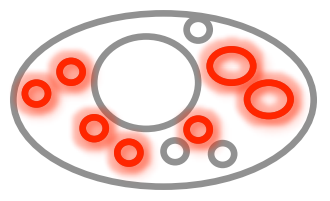

-5-15.min

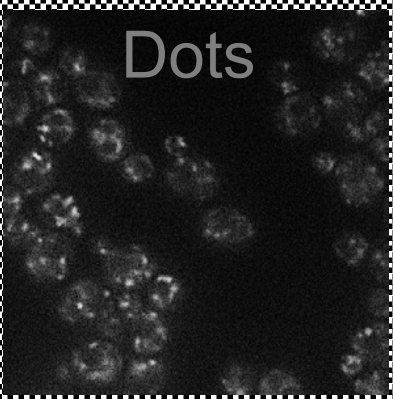

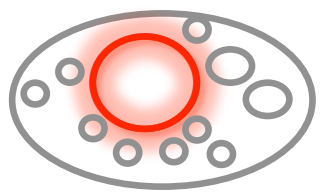

1 hour

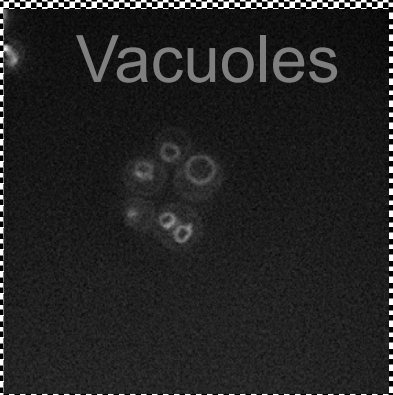

Figure IV.9 Dye structure of FM 4-64 and its schematic representation of the staining pattern in yeast cells. The Styryl dye, FM 4-64, has two lipophilic carbon tails and charged head regions. Its fluorescent property depends on the insertion of the hydrocarbon tail into the cell membrane and two aromatic rings by which the spectral properties are determined by the double bond in the center region (A). Increasing dye concentration $(0-20 \mu \mathrm{M})$ does affect the fluorescent intensities in the dye/liposome* complexes indicated as the solid squares. The open squares represent lacked liposomes with the dye (B). The emission spectra of FM 4-64 when bound to the surface of membrane (i.e. the dye-membrane complex means the status of the insertion of the tails into lipid bilayers) exhibits in $550-750 \mathrm{~nm}$ red-shifted by microscopy. The staining pattern of FM 4-64 can be divided into three patterns: Ring (plasma membranes), Dots (small punctate structures in endosome-like membranes) and Vacuoles (vacuolar membranes) at the represent time points $(\mathrm{C})$. Therefore, the dynamic fluids from the outer membrane into the 
vacuole can be pulse-chased with the FM 4-64 by endocytosis in yeast cells. Source: Dye structure of FM 4-64 and fluorescence intensities of dye-membrane complexes related to the dye concentration (A and B) were taken and adapted from the reference ( $\mathrm{Wu}$ et al., 2009). The asterisk represents that the liposome consists of $30 \%$ 1,2-dioleoyl-sn-glycero-3phosphoethanolamine (PE) / 70\% 1,2-dioleoyl-sn-glycero-3-phosphocholine (PC).

A
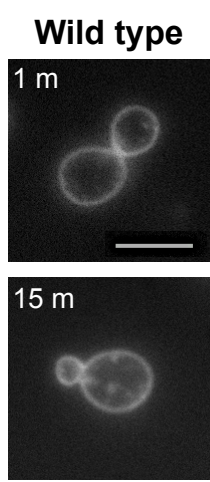

$30 \mathrm{~m}$

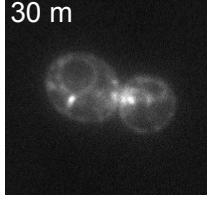

$50 \mathrm{~m}$
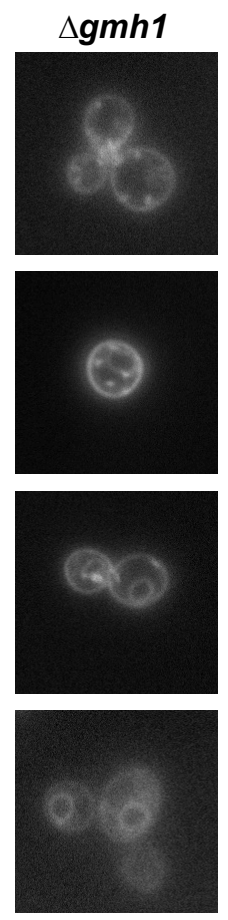

B
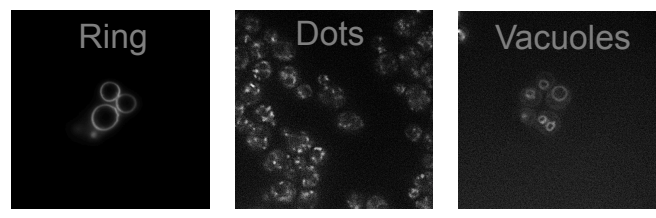

Wild type
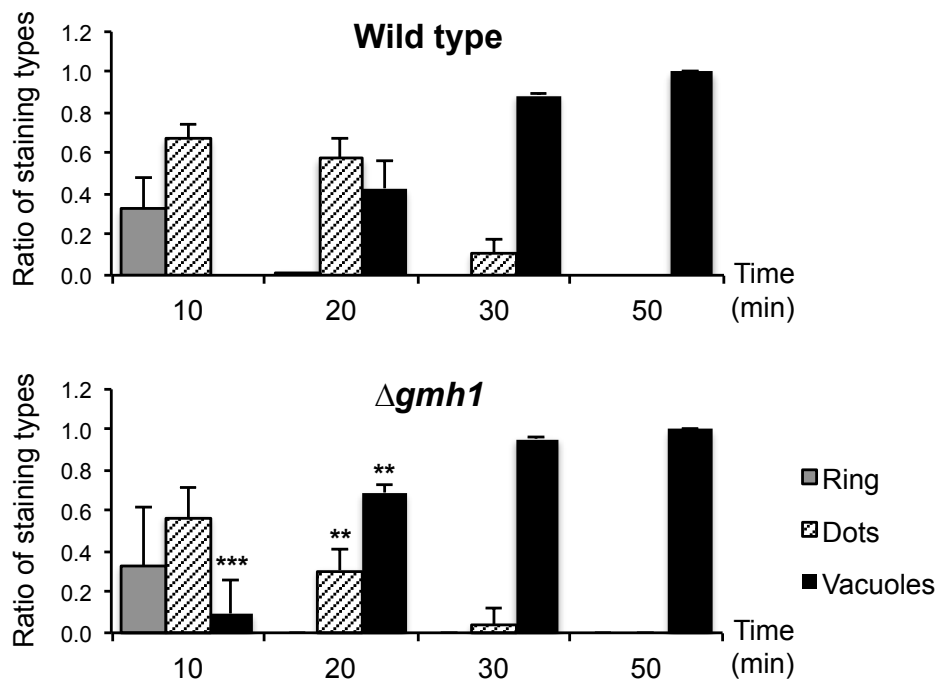

Figure IV.10 FM 4-64 dyes are rapidly reached to the vacuolar membranes at the early time points in gmh1 depletion. Live cell images indicating membrane dynamics were displayed at the indicated minutes (m) by FM 4-64. In gmhl mutants, the membrane dynamics was rapidly reached to the vacuolar membrane compared to the wild type at early time points (A). To obtain the numeric information, the images were divided into three patterns: Ring (plasma-outer membranes), Dots (small punctate structures in endosome-like membranes) and Vacuoles (vacuolar membranes), and by which the staining cells were measured as four time-intervals at 10 (1 to $15 \mathrm{~min}$ ), 20 (15 to $25 \mathrm{~min}$ ), 30 (30 to $40 \mathrm{~min}$ ) and 50 (50 to $60 \mathrm{~min}$ ) (B). Quantification of the staining types from four independent experiments was shown at each time point by measuring the membrane-associated staining shapes of the cells stained by FM 4-64 (B). Total number of cells divided by four groups as represent time-points (10, 20, 30 and 50) is shown as follows: wild type (943 cells at 10, 491 cells at 20,461 cells at 30 and 555 cells at 50) and gmh1 mutants 
(791 cells at 10, 937 cells at 20,617 cells at 30 and 705 cells at 50). Significant differences were shown in the represent time-points at $10(* * *, \mathrm{P} \leq 0.001)$ and $20(* * \mathrm{P} \leq 0.01)$, respectively. Error bars represent \pm s.e.m and data were analyzed by Student $t$-test. Scare bars, $5 \mu \mathrm{m}$.

\subsection{Recycling defects of vesicle components in $g m h 1$ mutants}

In the secretory pathway, carrier vesicles known as synaptobrevins or $v$-SNAREs are thought to facilitate its membrane recognition and vesicle fusion (Couve et al., 1995; Rothman and Warren, 1994; Takizawa and Malhotra, 1993). The $v$-SNAREs localized in the secretory vesicles play a role in vesicle docking, fusion and endo/exocytosis as vesicle-associated membrane proteins that interact physically with the targetingmembrane SNAREs ( $t$-SNAREs) from the plasma membrane (Baumert et al., 1989; Gerst et al., 1992; Gurunathan et al., 2000; Protopopov et al., 1993; Trimble et al., 1988). In addition, SNC1/2 $v$-SNAREs are involved in multiple trafficking steps and recycled to the trans-Golgi network via early endosomes in which SNC1/2 and GCS1 (ARF GTPaseactivating protein) interact in sorting cargo molecules back to the TGN through collaboration with coatomers in the Golgi-endosomal sorting activity (Hettema et al., 2003; Lewis et al., 2000; Robinson et al., 2006).

\subsubsection{SNC2-GFP localizes to the vacuolar-membranes in gmh1 mutants}

GMH1 was first identified as a geal-6 (ARF-GEFs) membrane-associated suppressor in yeast (Chantalat et al., 2003). Thus, if GMH1/UNC-50 functions as a retrograde trafficking factor shown in the synthetic lethality experiments (Table III.1B) in the Golgiendosomal interface (Fig. IV.1), the transport factors in the multiple trafficking steps are enhanced recruitments to the Golgi-endosomal interface on behalf of the GMH1/UNC-50 in its depleted conditions. As shown in the intracellular organelle markers in C. elegans and yeast (Fig. IV.3 and Fig. IV.4), gmh1/unc-50 mutants lead to an enhanced delivery to the vacuole/lysosome by the intracellular traffic-flow. In order to address close to the intracellular trafficking between the Golgi-endolysosomal interface on GMH1/UNC-50dependent pathway, we examined SNC2 labeled-GFP and overexpressed in yeast cells. The intracellular phenotypes of GFP-SNC2 observed was divided into three groups as 
follows: Vacuolar-membrane (VM), Punctates on VM (P.VM) and Spot-like in cells (Puncta). GFP-SNC2 was observed to be the vacuolar-associated membrane forms $(82 \pm$ $11 \%$ ) as the form of VM and P.VM in gmh1 mutants (Fig. IV.11A). Interestingly, GFPSNC2 in the VM seems as if a sausage chain and its punctate structures on vacuolar membrane (P.VM) were clustered to the vacuole in gmhl mutants. In contrast, the wild type in which most of cell types $(77 \pm 17 \%)$ was shown in punctate structures (Fig. IV.11A). Quantification analysis of the GFP-SNC2 was similar amount between wild type and gmh1 mutants by Western blots (Fig. IV.11B). As shown in VM and P.VM of the GFP-SNC2 in gmh1 mutants, we examined whether GFP-SNC2 colocalizes with FM 4-64 in the vacuolar membranes (Fig. IV.11C). It has been shown that the GFP-SNC2 was shown to be largely colocalized with FM 4-64 in the vacuolar membranes and obviously shown to be vacuolar-bound and membrane-attached types in gmhl mutants but not in the wild type (Fig. IV.11C). Together, these results suggest that GFP-SNC2 is recruited by GMH1-dependent pathway that would be considered as a cargo molecule and/or a fundamental factor involved in a manner dependent on GMH1sorting/trafficking pathway, supporting that a role of GMH1 is likely to be a regulator in recycling machinery components in the intracellular trafficking.

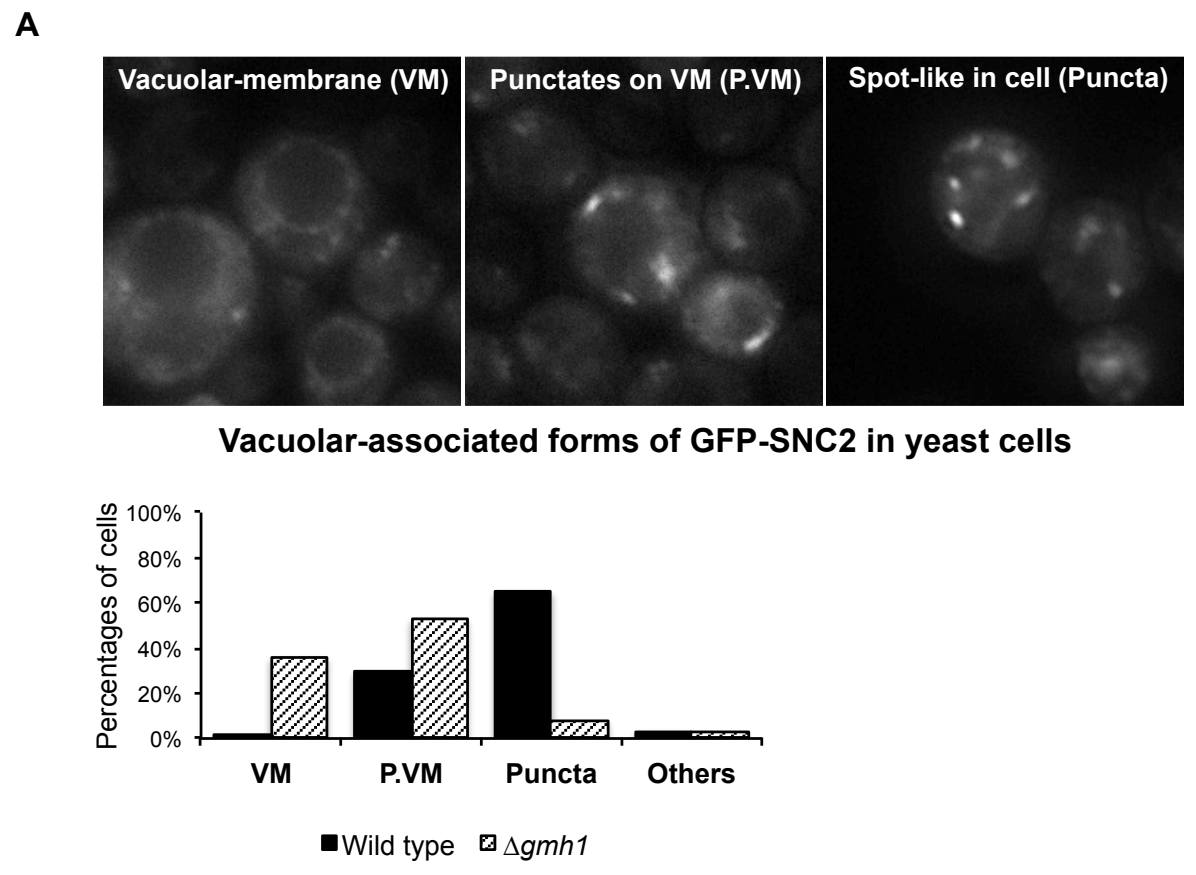


B
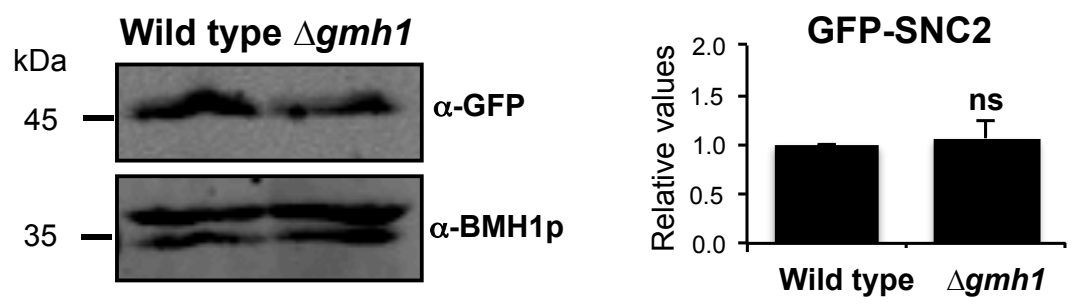

C

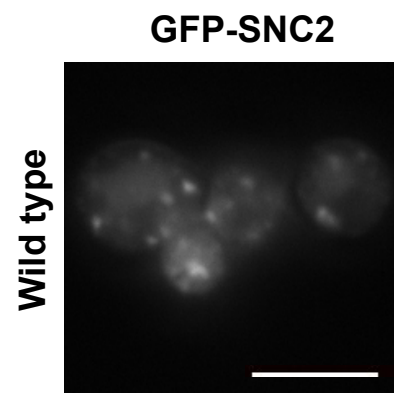

FM 4-64

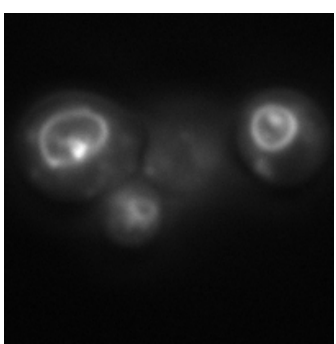

Merged
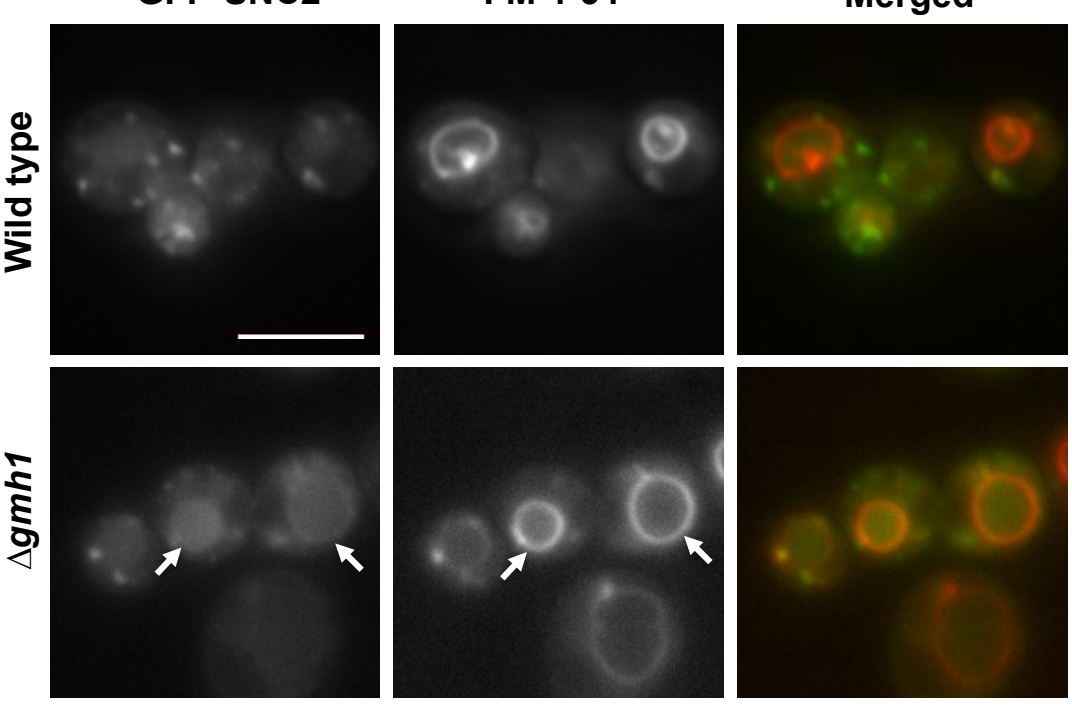

Figure IV.11 GFP-SNC2 appears to be staining-like on the vacuolar membrane in $g m h 1$ mutants. GFP-SNC2 were overexpressed and the results were divided into three groups as follows: Vacuolar-membrane (VM), Punctates on VM (P.VM) and Spot-like in cell (Puncta) in wild type and gmh1 mutants (A). Most of GFP-SNC2 in the VM seem as if a sausage chain and punctate structures clustered to the vacuolar membrane in gmhl mutant. Total number of cells used in this study was wild type (523) and gmh1 mutant (466) by three colonies as independent experiments (A). Quantification analysis was shown to be non-significant difference between wild type and gmh1 mutant in amount and the primary antibody used was anti-GFP and then normalized as a loading control, anti-BMH1p by Western blots. The ns represents non-significant by Student's $t$ test (B). Showing images appear to be colocalized to the vacuolar membrane in the gmh1 mutant between GFP-SNC2 and FM 4-64. Images taken after FM 4-64 staining for one hour were analyzed by microscopy $(\mathrm{C})$. Scale bars represent $5 \mu \mathrm{m}$. 


\subsubsection{Expression of TLG2-mCherry in yeast is not affected in gmh1 mutants}

The SNARE complexes are assembled to the target membrane-mediated vesicle recognition that drives vesicle fusion in eukaryotic cells and involved in many cellular processes as essential trafficking regulators (Jahn and Scheller, 2006). TLG2, v-SNARE, of yeast $S$. cerevisiae belongs to the syntaxin protein family and localizes to the TGN and endosomes (Abeliovich et al., 1998), which directly binds to vacuolar protein sorting (vps) VPS45, a yeast ortholog of Sec1/Munc18 (SM) protein, and is regulated with $v$ SNAREs SNC2 in the cellular expression levels (Nichols et al., 1998; Shanks et al., 2012). Due to the vacuolar membrane-targeting phenotypes of GFP-SNC2, the intracellular phenotypes of TLG2-mCherry were examined whether there are any defects of the intracellular phenotype in the absence of GMH1. As shown in Figure IV.12, TLG2-mCherry was overexpressed into the strains wild type and gmhl mutants. Both were shown to be similar aspects unlike the intracellular phenotypes of GFP-SNC2 which had appeared to be vacuolar membrane-targeting in the GMH1-deletion (Fig. IV.11). In the synthetic lethal assays, $t$-SNAREs $\operatorname{tg} 1$ and $s y n-16$ (tm1506) have been shown to be viable with unc-50 (x47) mutants in yeast and C. elegans, respectively, even the triple combination mutants of unc-50 (x47); syn-13 (tm2037); syn-16 (tm1506) appeared to be normal growth phenotypes (Table III.1). This indicates that UNC-50/GMH1 seems not to be redundant in functioning SNAREs complex either in the anterograde or retrograde transport. This means that it may exist largely redundant in both pathways with distinguished mechanisms because gmhl mutants do not cause phenotypic defects of TLG2-mCherry by microscopy. 


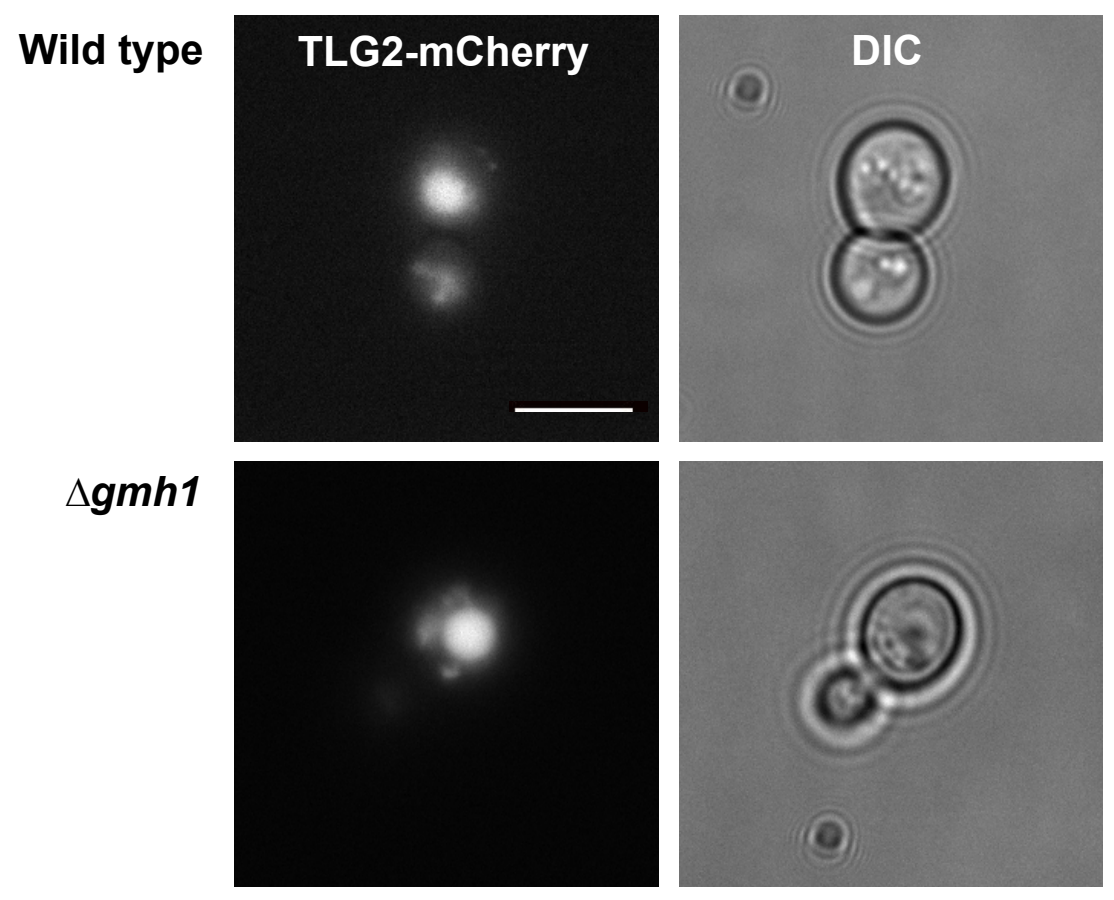

Figure IV.12 mCherry-TLG2 in yeast cells does not affect in the gmh1 mutants. The $t$ SNARE TLG2 tagged-mCherry was transformed and overexpressed in both wild type and gmh1 mutants $(\Delta g m h 1)$. They are shown to express similar and no significant difference between two strains wild type (BY4741) and gmh1 mutants. The images were taken by a Coolsnap HQ camera of a Delta Vision RT (Applied Precision) microscopy by using 100x/0.35-1.5 Uplan Apo objective with filter sets of mCherry (Ex: 575/25, Em: 632/60) and differential interference contrast (DIC) fields were shown in wild type and $\Delta g m h 1$, respectively. Scale bars represent 5 $\mu \mathrm{m}$.

\subsection{Requirement of Gmh1p for the trafficking of the yeast CLC protein Gef1p}

Growth phenotypes of an organism depend on expressed genomes and growth conditions. In yeast, the relevance of genes in the intracellular trafficking can also be determined by yeast growth phenotypes. GMH1 is known as a membrane-mediated ARFGEFs GEA1/2 interaction partner and its overexpression can rescue the thermosensitive growth defect of gea 1-6 in yeast growth experiments and it would be conceivable as a transmembrane receptor for recruitment of ARF-GEFs GEA1/2 to the membrane of Golgi (Chantalat et al., 2003). 


\subsubsection{Growth phenotypes and localization of GEF1-GFP in GMH1-depleted strains}

To find out the relative molecules on the gmhl-dependent manner, yeast growth phenotypes were tested as shown in Figure IV.2. We found that the definitive growth phenotypes of gmhl-depleted strains appeared to grow better than wild type on the YP.Gly/Lac plates regardless of iron-limited and/or starvation conditions (Fig. IV.2D-G). In order to see the growth interactions between GMH1 and GEF1 as a yeast ortholog of the CLC proton-chloride transporter related to the iron metabolism (Braun et al., 2010; Greene et al., 1993), we investigated GEF1 in the growth phenotypes, expression levels and localization in the intracellular compartments. The conserved integral Golgi membrane protein, GEF1, is likely to be in the endo-lysosomal pathway for acidification of vesicle and balancing the proton-chloride exchange in the intracellular compartments (Gaxiola et al., 1999; Gaxiola et al., 1998; Scheel et al., 2005; Schwappach et al., 1998; Wachter and Schwappach, 2005). Even though GMH1 does not seem to be directly involved in iron metabolisms through the growth phenotypes of cellular expressions (Fig. IV.2), GFE1 might be regulated by GMH1 as a minor factor in cellular metabolic conditions.

In order to test whether GEF1 is affected in gmh1 mutants, $\mathrm{N}$ - and C-terminally tagged GFP (GFP-GEF1 and GEF1-GFP in P416MET25) (Braun et al., 2010; Schwappach et al., 1998) were overexpressed and observed in wild type (BY4741) and gmh1 mutant strains by microscopy (Fig. IV.13). Cellular expression intensity of the GFP-GEF1 and GEF1GFP were relatively dimmed in gmhl mutants (Fig. IV.13C). To access certain genetic interaction in regulating GEF1 on GMH1-dependent pathway, yeast growth phenotypes were assessed by spot serial dilutions on YP.Gly/Lac plates. The idea was that GMH1 would regulate GEF1 in the intracellular compartments. In the yeast phenotypes, GMH1deletion strains appeared growth better than wild type on non-fermentable and ironlimited conditions, even though the growth phenotypes of gmh1 mutants were shown to grow better in both cases with/without iron chrators on YP.Gly/Lac (Fig. IV.2D-E). When overexpressed-GEF1 (GFP-GEF1 and GEF1-GFP), the growth phenotypes of the spot dilutions were observed in the wild type (WT* means that GMH1 exists in the cells). The growth phenotypes of WT* were shown to grow better than the wild type where the empty vector (P416MET25) was overexpressed. As shown in Figure IV.13D, the spot 
dilution lines of red-arrows marked were shown to be growth better which appeared to 1.7-fold in GFP-GEF1 and 1.3-fold in GEF1-GFP, respectively, remarked by surface plots and relative intensities dashed-red lines. The difference of growth phenotype was in subtle than expected, indicating that cellular growth phenotypes by balancing metal-ions homeostasis may be affected by tight regulation in the intracellular organisms. These results suggest that cellular expression of GEF1, GFP-GEF1 and GEF1-GFP, is affected by GMH1 in yeast cells, even though in subtle. 
A

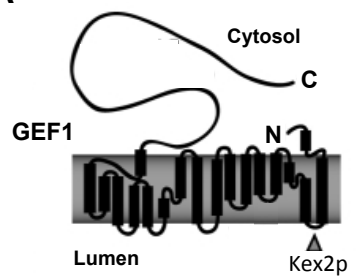

C

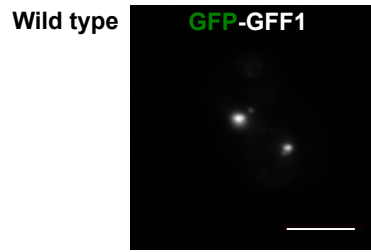

$\Delta g m h 1$

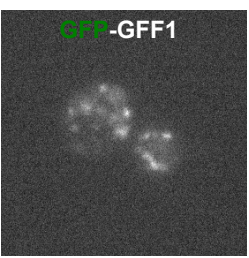

B
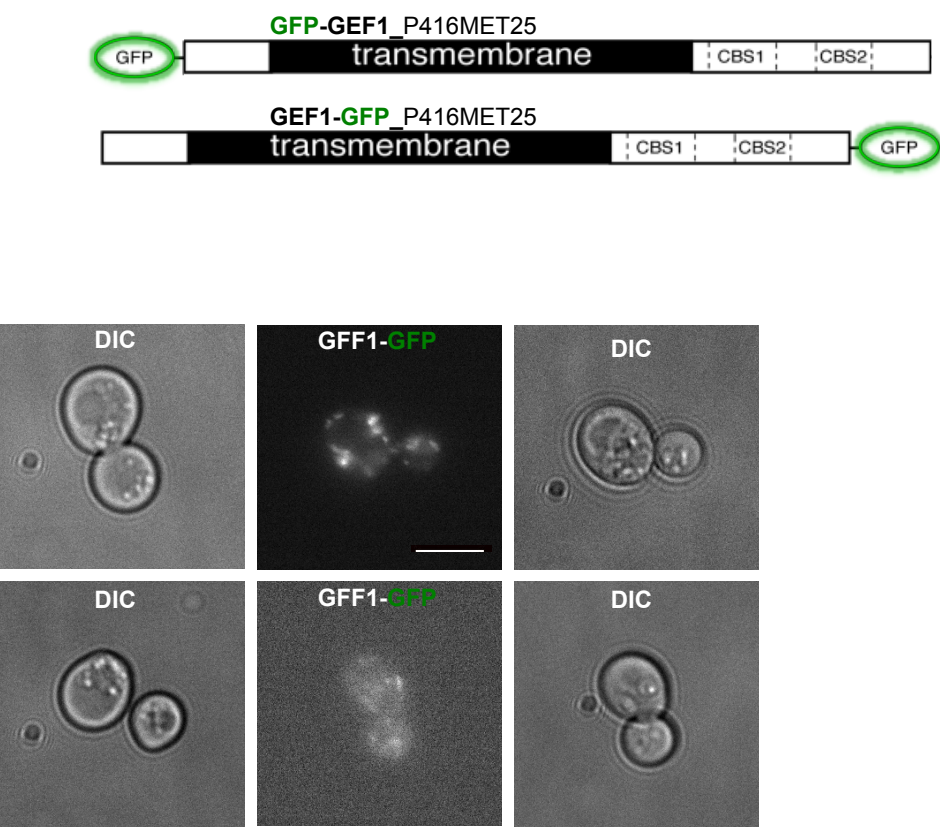

D
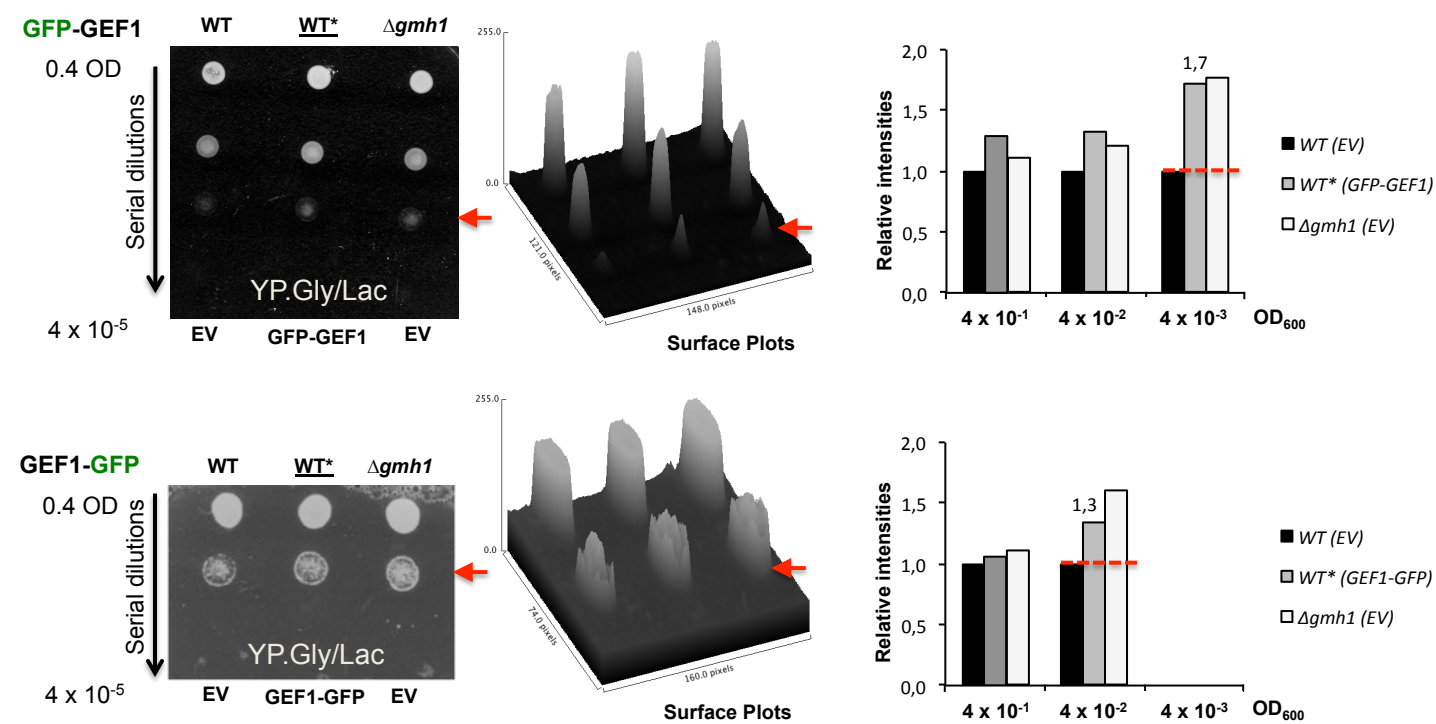

Figure IV.13 Cellular-overexpression phenotypes of GEF1 tagged-GFP, GFP-GEF1 and GEF1-GFP, in wild type are slightly rescued to gmh1 mutant strains. Structure of GEF1, based on the crystal structure of $E$. coli, in which a smaller $\mathrm{N}$-terminal and large C-terminal fragment faces in cytosol and the cleavage site by Kex2p, that processes in the late-Golgi, is indicated in the lumen (A). Schematic diagrams represent the N-terminal tagged-GFP of GEF1, GFP-GEF1, and C-terminal tagged-GFP of GEF1, GEF1-GFP (B). Cellular expression phenotypes of GFP-GEF1 and GEF1-GFP show less intensity of the fluorescence in $g m h 1$ 
deletion strains by microscopy $(\mathrm{C})$. The images were taken by a Coolsnap HQ camera of a Delta Vision RT (Applied Precision) microscopy by using 100x/0.35-1.5 Uplan Apo objective with filter sets of GFP (Ex: 470/40, Em: 525/50) and differential interference contrast (DIC) fields were shown in wild type and $\Delta g m h 1$, respectively. Scale bars represent $5 \mu \mathrm{m}(\mathrm{C})$. Yeast growth phenotypes by spot serial dilutions from 0.4 OD (optical density, $\mathrm{OD}_{600} \mathrm{~nm}$ ) to $4 \times 10^{-5} \mathrm{OD}$ (D). WT* represents the overexpression of GFP-GEF1 and GEF1-GFP in wild type and EV indicates the overexpression of the empty vector, P416MET25, as a control in wild type and gmh1 mutants $(\Delta g m h 1)$. The arrows and dashed lines, red color, indicates the difference of growth phenotypes (D). Source: The pictures (A and B) were taken and modified from the reference (Braun et al., 2010; Schwappach et al., 1998).

\subsubsection{GMH1 deletion induces low intensity of GEF1-GFP}

In addition to the cellular expression phenotypes of GFP-GEF1 and GEF1-GFP shown to express less intensities of the fluorescence in gmhl deletion strains by microscopy (Fig. IV.13C), the six strains used for growth phenotypes (Fig. IV.2), wild type (BY4741), $\Delta g m h 1, \Delta g e f 1, \Delta g m h 1 ; \Delta g e f 1, \Delta p e p 4, \Delta f e t 3$ and $\Delta g m h 1 ; \Delta p e p 4$ were transformed with GFP-GEF1 and GEF1-GFP, respectively, and Western blots were performed to reveal biochemical protein levels in each strain. It has been interestingly shown that the overexpression of GFP-GEF1 and GEF1-GFP representing the full-length at around 100 $\mathrm{kDa}$ (Wachter and Schwappach, 2005) was rarely detected in $\Delta g m h 1$ and $\Delta f e t 3$ strains (Fig. IV.14A). The $\Delta g e f 1$ and $\Delta g m h 1 ; \Delta g e f 1$ strains appeared to be rescued in the blots, in particular, when overexpressed GEF1-GFP, the C-terminally tagged GFP of GEF1. As you can see the structure of GEF1 (Fig. IV.13A), the C-terminal end of GEF1 containining two copies of cystathione $\beta$-synthetase (CBS) domains is largely indicated in cytosol.. It has been known that the CBS domains bind to ATP as an energy sensor of adenosine-ligands in the C-terminus of CLC-5 in mammalian (Meyer et al., 2007; Scott et al., 2004). This suggests that the GEF1-GFP is likely to be recruited by the cytosolic effectors in the intracellular compartments and stably kept in the biochemical approach detecting protein levels. In contrast, the N-terminal end of GEF1 has the proteolytic cleavage sites by Kex2, which processes in the post-Golgi, at the first luminal loop (Fig. IV.13A). Based on the structure of GEF1, we observed the C-terminal tagged GFP of GEF1 (GEF1-GFP) with the following experiments Western blots. 
In the previous study, unc-50 mutants in C. elegans lead to mis-sorting of Lev-nAChRs into the lysosome, by which the assembled receptors of the Lev-nAChRs were back to detectable intensities by Western blots in the double mutants unc-50 and cup-5 essential for lysosomal formation (Eimer et al., 2007). These results led us to test double-deletion mutant pep4;gmh1. PEP4 is known as a vacuolar protease essential for protein degradation in yeast cells (Teichert et al., 1989). As shown in Figure IV.14B, the GEF1GFP in the pep4 single mutant was shown to be similar expression levels to the wild type. In the result, the relative concentration of the GEF1-GFP in pep4;gmh1 mutants was much higher which means GEF1-GFP would be mis-routed to the vacuole/lysosome pathway in the absence of GMH1 (Fig. IV.14B). This suggests that GEF1-GFP may be regulated through a GMH1-dependent manner so that in the same way certain molecules would not be recycling in the intracellular organelles and induced to the vacuolar/lysosomal targeting as shown in both experiments (Fig. IV.3 and Fig. IV.4).

A

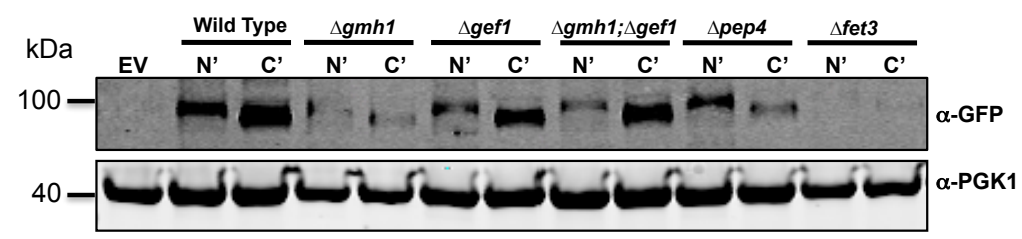

Yeast strains

N' = GFP-GEF1 in P416MET25

$\mathbf{C}^{\prime}=$ GEF1-GFP in P418MET25

EV = empty vector, $\mathrm{P} 416 \mathrm{MET} 25$

B
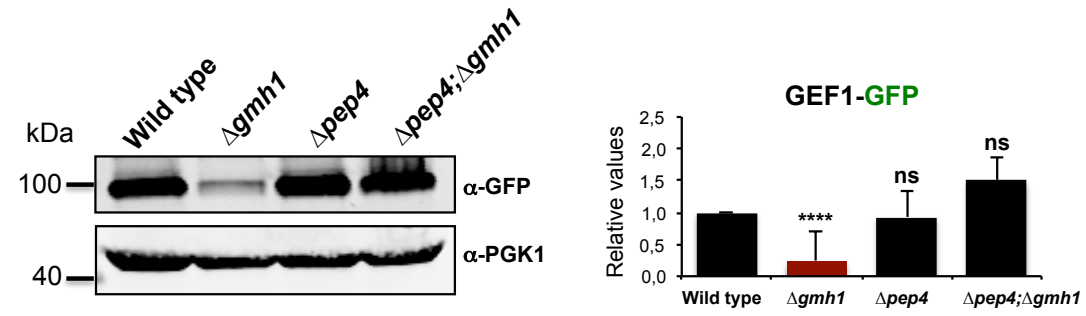

Figure IV.14 GMH1 deletion induces low intensity of GEF1-GFP, yeast CLC protein. The six strains, wild type (BY4741), $\Delta g m h 1, \Delta g e f 1, \Delta g m h 1 ; \Delta g e f 1, \Delta p e p 4, \Delta f e t 3$ and $\Delta g m h 1 ; \Delta p e p 4$, which were assessed by yeast growth phenotypes (Fig. IV.2), were transformed with GFP-GEF1, the N-terminal tagged GFP of GEF1, and GEF1-GFP, the C-terminal tagged GFP of GEF1, respectively, in the plasmid P416MET25 (Braun et al., 2010; Schwappach et al., 1998). The GFPGEF1 and GEF1-GFP of the full length were much less detected in the GMH1-depleted strains (A). The intensity of GEF1-GFP was confirmed by Western blots. Three independent experiments were performed in wild type (BY4741), $\Delta g m h 1, \Delta p e p 4$ and $\Delta p e p 4 ; \Delta g m h 1$. GMH1 
deletion strains were much destabilized-intensity of GEF1-GFP, but the intensity of GEF1-GFP was shown to back higher intensity than wild type and $\Delta p e p 4$ in a double mutant, $\Delta p e p 4 ; \Delta g m h 1$ (B). The full length of GFP-GEF1 and GEF1-GFP is indicated at around $100 \mathrm{kDa}$ by using antiGFP mouse monoclonal B-2 $(0.2 \mathrm{mg} / \mathrm{ml}$, Santa Cruz $), 1: 1000$ dilution and anti-PGK1 (Phosphoglycerate Kinase 1) mouse monoclonal 22C5 (1 mg/ml, Molecular Probes), 1:1000 dilution, was used as a cytosolic control to be normalized. Error bars represent the standard error of the mean $( \pm$ s.e.m). Asterisks indicate significant between the wild type and gmhl mutants as follows: ****, $\mathrm{P} \leq 0.0001$; ns, non-significant by Student's $t$ test.

\subsection{Secretion defects of ssGFP from muscles in unc-50 mutants}

Apart from the levamisole-resistant phenotype of unc-50 mutants in C. elegans, the distal tip cell (DTC) migration defects of the posterior gonads were observed in unc-50 mutants that cause gonad migration defects similar to the defective phenotype of mig-17 mutants (Ling Luo, PhD dissertation, 2010). MIG-17, a soluble metalloprotease, is secreted from the body wall muscle cells as a proform with a glycosylated N-terminal prodomain and localizes to the gonadal basement membranes as a metalloprotease (MP) domain, a disintegrin (DI) domain and a protease and lacunin (PLAC) domain at the Cterminal (Ihara and Nishiwaki, 2007). We found in the previous study that MIG-17 and UNC-50 in the same genetic pathway play a role to regulate DTC migration and, in unc50 mutants, MIG-17 can be expressed and normally localized at the gonadal basement membrane as revealed by immune-staining and Western blots, respectively. However, when overexpressed MIG-17::VENUS in unc-50 mutants, the ratio (3:1) analyzed between the proform and the mature form of MIG-17 was different from the ratio (1:1) in wild type, indicating that MIG-17 might be trapped in the body wall muscles in the absence of UNC-50 in muscles. Thus, we tested the secretion from the body wall muscles, in order to verify the secretion pathway on UNC-50-dependent, by using the transgenic worm line, arIs 37 [pmyo-3::ssGFP], expressing signal sequence-GFP fusion protein (ssGFP) from muscle cells to the body cavity in which the ssGFP is taken up by coelomocytes, six macrophage-like scavenger cells in C. elegans, in the pseudocoelom (Fig. IV.15) (Fares and Greenwald, 2001; Girard et al., 2007; Grant and Sato, 2006). Before testing the secretion of ssGFP in $u n c-50$ mutants, we confirmed that secretion of 
MIG-17 from muscles was not affected in secretion of ssGFP from muscles as well as secretion of ssGFP from the body wall muscles was also not related to the gonad morphological defects by mis-migration of DTCs.

In the secretion tests of ssGFP, the wild type in coelomocytes was fulfilled inside as green signals, ssGFP, whereas in unc-50 mutants the ssGFP was much less in coelomocytes. Instead, it was shown to be trapped in the body wall muscles (Fig. IV.16A). The relative signal intensity of ssGFP between coelomocytes and body wall muscles was quantitatively analyzed and shown to be significantly different in coelomocytes and nearby muscles between the wild type and unc-50 mutants (Fig. IV.16B). This supports that UNC-50 may be required for the proper secretion of MIG-17 from the body wall muscles. It is a rising possibility that, in unc-50 mutants, MIG-17 can be mis-sorted to the lysosomal pathway. These results suggest that UNC-50 is involved in trafficking pathway as a secretary or general factor in the Golgi-endolysosomal compartments.

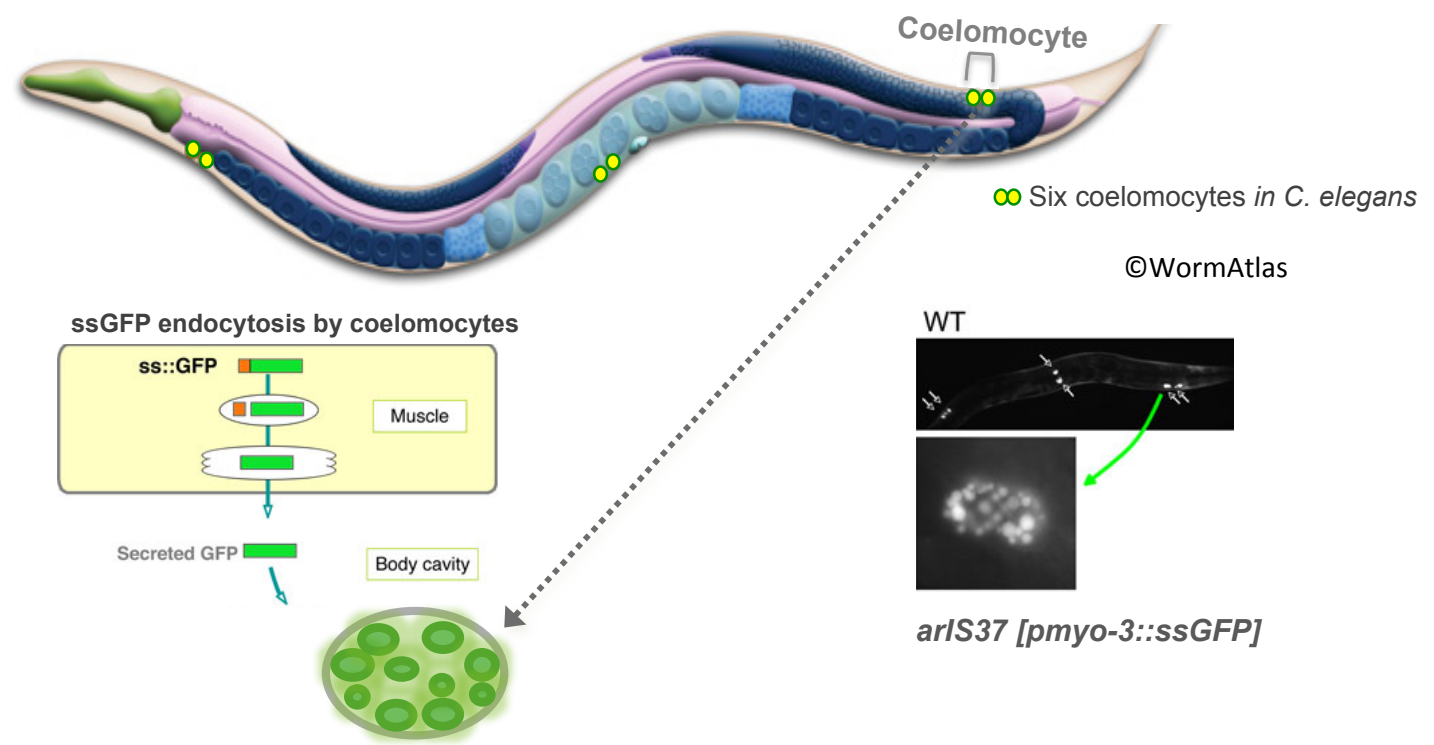

Figure IV.15 Schematic representation of endocytosis by coelomocytes of adult hermaphrocites in $\boldsymbol{C}$. elegans. The signal sequence-tagged GFP, ssGFP, is produced in muscles and internalized into six coelomocytes in C. elegans. The transgenic worm strain arIS37 [pmyo$3:: s s G F P]$ shows ssGFP in coelomocytes (Fares and Greenwald, 2001; Grant and Sato, 2006). Source: Pictures used were taken and modified from the references (Fares and Greenwald, 2001; 
Grant and Sato, 2006) and the image of C. elegans, coelomocytes shown in adult hermaphrocites, was taken from WormAltras.org.

A
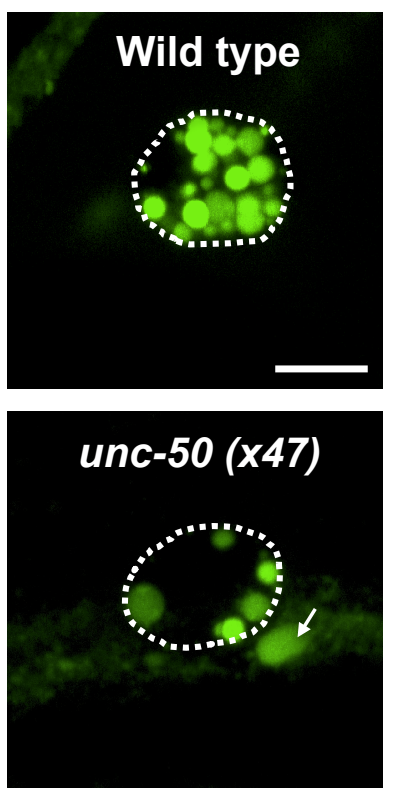

B

\section{ssGFP in coelomocytes}

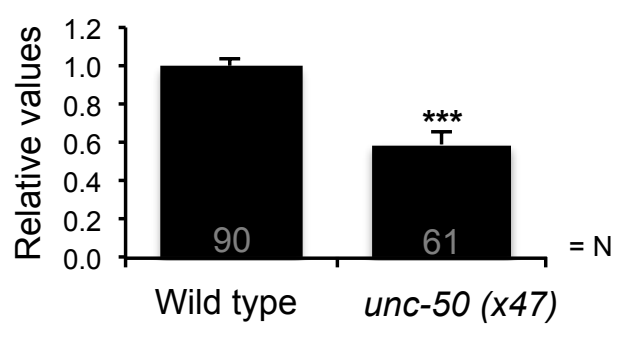

ssGFP in muscles

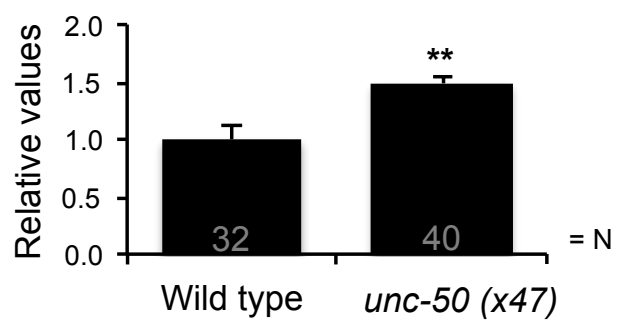

Figure IV.16 The signal sequence GFP fusion protein (ssGFP) from the body wall muscles was not properly secreted due to be trapped in the muscles in unc-50 mutants. Confocal images of the ssGFP in both wild type and unc-50 (x47), in which the strain arIS37 [pmyo$3:: s s G F P]$ secrets ssGFP from the muscle cells to the body cavity, are shown to be taken up by the immune system cell coelomocytes of $C$. elegans. White arrow shows ssGFP in the muscles in unc-50 (x47) and scale bars represent $5 \mu \mathrm{m}$ (A). Comparison of the relative intensities of the ssGFP in wild type and unc-50 (x47) was shown in both coelomocytes and muscles nearby posterior side of the gonad. Error bars mean the standard error of the mean ( \pm s.e.m) by three independent experiments. The significance of the different values between wild type and unc-50 was shown as asterisks (**, $\mathrm{P} \leq 0.01 ; * * *, \mathrm{P} \leq 0.001)$ by Student's $t$ test $(\mathrm{B})$. 


\subsection{Interspecies-expression of $\mathrm{gmh} 1$, a yeast and human ortholog of unc-50, does not rescue and localize in $C$. elegans}

Since UNC-50 has been identified in various species, we tried to confirm whether a yeast and human ortholog of UNC-50, GMH1 and hGMH1, are able to be functional in the body wall muscles of $C$. elegans. This idea was that it might be able to interact or be functional with the yeast/human orthologs of UNC-50 as a traffic-machinery to be alternately expressed in interspecies. It has also been previously reported that in the interspecies Drosophila subunits of nAChRs can be reconstituted and expressed in Xenopus oocytes with the subunits of vertebrate (Bertrand et al., 1994; Ihara et al., 2003). In this study, as a trafficking machinery, we tested UNC-50/hGMH1/GMH1 to be expressed in the interspecies.

In order to test whether the hGMH1 and GMH1 can be functional in C. elegans, we overexpressed the hGMH1::mYFP and GMH1::mYFP under the muscle specific promoter myo-3 into $C$. elegans, respectively. Unlike the previous studies about the expression of the hybrid-nAChRs in Xenopus oocytes, they did not restore the phenotype of levamisole-sensitivity in both hGMH1::mYFP and GMH1::mYFP in C. elegans. The overexpression of $C$. elegans UNC-50::mYFP in the body wall muscles did normally rescue the phenotype levamisole-sensitive and show much improved movement of the body bends like the wild type (Fig. IV.17A and B). Moreover, the body bends per minute and the localization in the intracellular compartments of hGMH1::mYFP and GMH1::mYFP were not shown to be restored at all in C. elegans (Figure IV.17A and B). In contrast, UNC-50::mYFP of C. elegans was explicitly rescued in the body bends, the phenotype of levamisole-sensitivity and the localization as shown to be colocalized with alpha-mannosidase II as a medial Golgi marker (Mans::tagRFP) (Fig. IV.17C). The localization of hGMH1::mYFP and GMH1::mYFP was not indicated in the body wall muscles of $C$. elegans by using confocal microscopy. These data indicate that hGMH1::mYFP and GMH1::mYFP may not be functionally expressed and properly localized in $C$. elegans. The foreign proteins and machineries may be subsequently degraded in C. elegans. 
A

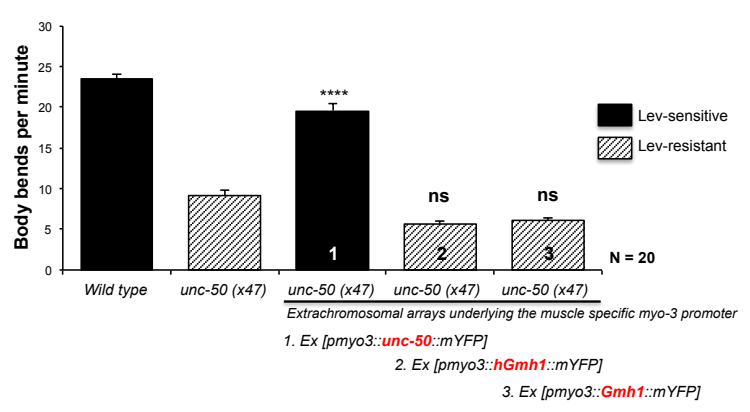

C unc-50(x47)_Ex[pmyo3::unc-50::mYFP] in C. elegans

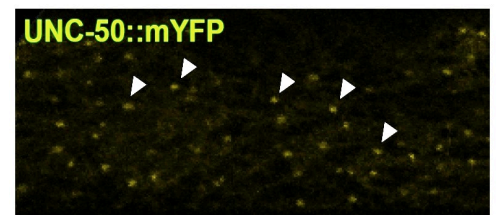

B

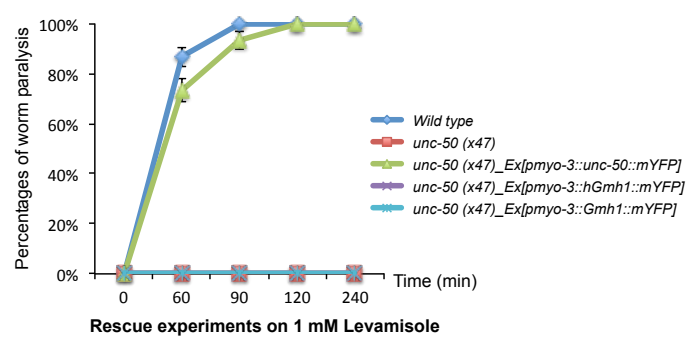

Figure IV.17 Gmh1, the yeast and human ortholog of $u n c-50$ is not able to be functional in the body wall muscles in $\boldsymbol{C}$. elegans. Interspecies-expression of unc-50 and gmh1 in the body wall muscle of $C$. elegans was tested by extrachromosomal arrays with three constructs as follows: 1. Unc-50 tagged-mYFP (UNC-50::mYFP) of C. elegans_Ex [pmyo3::unc-50::mYFP] 2. The human ortholog of unc-50,hGmh1_Ex [pmyo3::hGmh1::mYFP] 3. The yeast ortholog of unc-50, Gmh1_Ex [pmyo3::Gmh1::mYFP]. Each construct was expressed in the body wall muscles and functionally evaluated in C. elegans. Body bends per minute and phenotype tests on $1 \mathrm{mM}$ levamisole were shown and the UNC-50::mYFP of C. elegans was rescue, but not with one of the human $(h G m h 1)$ and yeast $(G m h l)$ (A and B). Confocal pictures showing the normal localization of UNC-50::mYFP as rescued in C. elegans and colocalization of UNC-50::mYFP with the medial Golgi marker, $\alpha$-mannosidase II labeled with tagRFP, in young adult body wall muscles in C. elegans (white arrowheads) (C). The yeast and human ortholog of unc-50, Gmh1 and $h G m h 1$, were not able to be functional and localized in the body wall muscles in C. elegans. Scale bar, $5 \mu \mathrm{m}$. 


\section{DISCUSSION}

\subsection{UNC-50/GMH1 acts as a redundant factor in retrograde transport from endosomes to the Golgi}

We have studied that on the subtype-specific trafficking manner how UNC-50 is required for intracellular trafficking in two organisms, C. elegans and $S$. cerevisiae. Although previous studies have placed UNC-50 and Lev-nAChRs as a specific pair for the receptor trafficking to the cellular surface (Eimer et al., 2007), we propose that the UNC-50 is not only for Lev-nAChRs trafficking but also plays a role in an unspecific cargo transport as a retrograde trafficking factor from endosomes to the TGN as shown in Table IV.1. In addition, UNC-50 has a function in controlling traffic-flow of machinery components mediated by RAB and ARF GTPases as intracellular organelle markers in the endo-lysosomal pathway (Fig. IV.3 and IV.4).

An evolutionarily conserved protein UNC-50 was initially observed in an inner nuclear membrane, ER, and Golgi apparatus in previous studies (Chantalat et al., 2003; Eimer et al., 2007; Fitzgerald et al., 2000). In order to find out the reason for the existence of UNC-50 in all eukaryotic cells, we sought to determine its role in sub-cellular interfaces and became aware of intracellular features which are likely to be a cargo specific or unspecific pathway on UNC-50-dependent. Among the eukaryotic cells, plant Arabidopsis thaliana and yeast $S$. cerevisiae do not have nAChRs, but they are likewise highly conserved the protein UNC-50 in species. Therefore, UNC-50 may play a fundamental role in the intracellular compartments in where UNC-50 localizes.

Our synthetic lethal experiments by the mutant combination between the trafficking factors of the Golgi-endosomal interface and unc-50/gmhl showed that the retrograde trafficking factors GARP, COG, retromer complex and RAB-6 appeared to be lethal with unc-50/gmh1 mutants whereas the anterograde trafficking factors were viable and normal growth (Fig. V.1). It has been known that the retrograde trafficking factors disrupt protein retrieval to the TGN from endosomes and disturb the recycling of SNARE complexes in the TGN-endosomes (Conibear et al., 2003; Hayes et al., 2009; Quenneville et al., 2006; Rojas et al., 2008; Siniossoglou and Pelham, 2001). These reports support that UNC-50 
may act as an unspecific/fundamental factor to be recycling protein components in the retrograde trafficking. In addition, as a membrane-mediated ARF-GEFs GEA1/2 interaction partner, GMH1 has only been proposed as a transmembrane receptor for recruiting the ARF-GEFs GEA1/2 at the early-Golgi complex (Chantalat et al., 2003). However, GMH1 does not seem to be functionally essential in cellular processes due to alternative factors to regulate membrane trafficking in the TGN. The retrograde factors would provide redundant roles to compensate each other when individual sorting factors are lost in close proximity. In this respect, recycling mechanisms and certain factors would also be conserved in similar aspects in eukaryotic cells (Donaldson and Segev, 2010; Seaman, 2005).

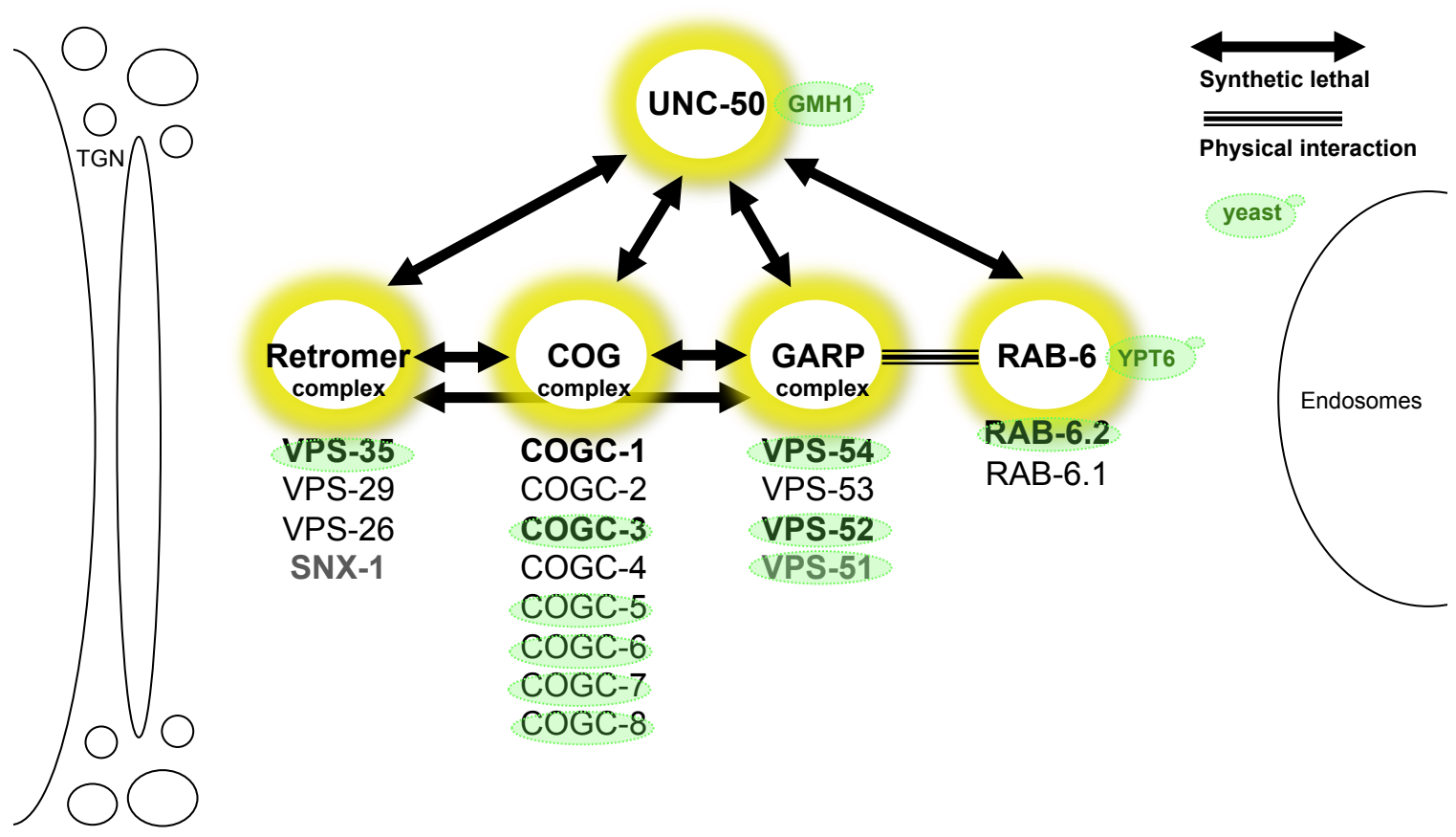

Figure V.1 Schematic representation of the synthetic lethality between the mutants of the unc-50/gmh1 and the retrograde trafficking machineries in both yeast $S$. cerevisiae and nematode $C$. elegans. unc-50/gmh1 mutants cause synthetic lethal in combination with the retrograde trafficking factor mutants GARP, COG, Retromer complex and RAB-6 involved in the cargo retrival of the endosome-to-Golgi. The synthetic lethal phenotypes either appeared in the genetic combinations between the retrograde trafficking factors as well as a physical interaction. 


\subsection{UNC-50/GMH1 leads to enhanced recruitments to the endosome-to-lysosomal compartments}

The intracellular phenotypes of the unc-50/gmh1 mutants are sufficient to explain that intracellular organelle markers fluorescent-labeled APT-9, RAB-5, RAB-7 and a lysosomal protein CTNS-1 were probably recruited by UNC-50-dependent because they were shown to be increased punctate structures and strong intensity in unc-50 mutants (Fig. IV.4). It is likely to fail to control the intracellular traffic-regulation due to the absence of UNC-50. This suggests that UNC-50 might be necessary to get ready for tight traffic-regulation in the endo-lysosomal pathway. It is consistent that the yeast strains lacking GMH1 were shown to be enhanced recruitments to the vacuolar membrane targeting shown in CHC1-RFP, SNF7-RFP and GFP-SNC2 (Fig. IV.3). Moreover, we found that UNC-50 functions in secretion as a regulator of ssGFP in muscles (Fig. IV.16), supporting that UNC-50 may operate an efficient secretion of MIG-17 that causes gonadal migration defects as a visual phenotype. As demonstrated by membranedynamics, the endocytic-flow aspects to the vacuolar membrane appeared to be rapidly reached in GMH1 deletion strains at the early time stages (Fig. IV.10), which indicates that UNC-50/GMH1 does affect in general transport pathway as an unspecific/regulating manner.

\subsection{GMH1 is a novel recycling factor of the vesicle fusion machinery SNC $v$ - SNARES}

To make an efficient traffic-flow in intracellular organelles, cargoes and machinery components are needed to be matched each other in sorting and trafficking. The machinery components SNC1/2 v-SNAREs are involved in multiple trafficking steps and recycled between the TGN and endosomes (Hettema et al., 2003; Lewis et al., 2000; Robinson et al., 2006). We found that the SNC2-GFP was shown to the vacuolar membrane-bound forms in gmhl mutants instead of recycling in the intracellular compartments. As the consistent results with the retrograde trafficking factors, it has been known that the vps54 mutants of GARP complex lead to recycling defects of the SNC1 vSNARE from endosomes back to the TGN retrieval (Quenneville et al., 2006), suggesting 
that UNC-50/GMH1 as a membrane-mediated trafficking factor might be required for recycling proteins as one of machinery components in the endosomal-lysosomal/vacuolar compartments (Fig. V.2) (Donaldson and Segev, 2010).

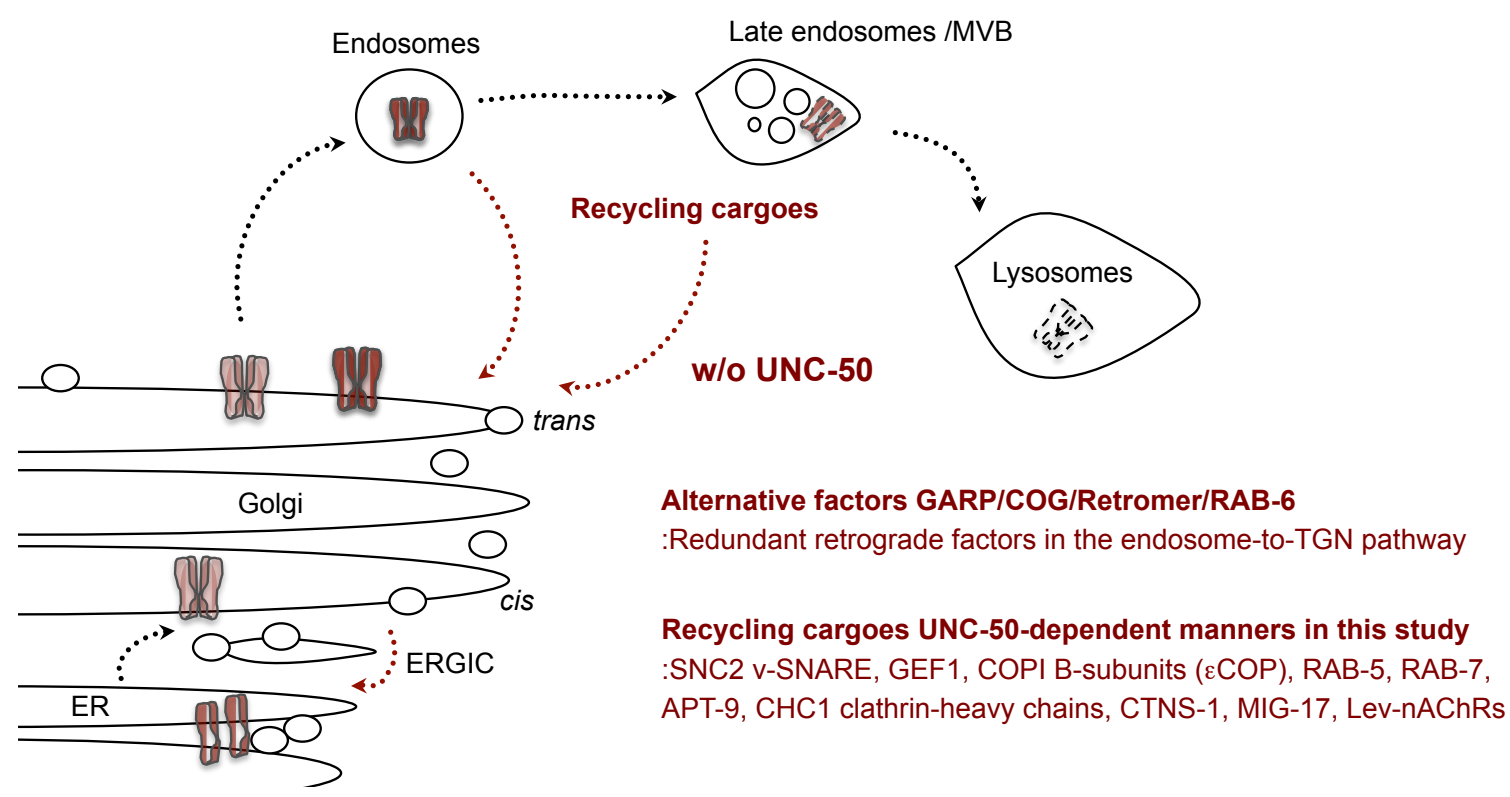

Figure V.2 UNC-50/GMH1 is a novel recycling factor in the retrograde pathway, which regulates in a redundant manner of the parallel pathway with the complex of GARP/COG/Retromer and the small GTPase RAB-6. In the absence of UNC-50/GMH1, the multimeric proteins such as Lev-nAChRs follow the endolysosomal pathway that leads to enhanced recruitments of the membrane-mediated machinery components SNC2, RAB5/7, APT9 and coated proteins COPI/Clathrin on the UNC-50/GMH1-dependent manners.

\subsection{GMH1 may involve indirect regulation of cargo molecules}

Based on the depletion phenotype of UNC-50/GMH1, gmh1 mutant strains have a resistance on non-fermentable conditions in yeast growth (Fig. IV.2). GMH1 would be involved in cellular homeostasis that has to be tightly regulated in intracellular organisms. Our finding showed that GEF1-GFP, required for iron homeostasis in the endo-lysosomal pathway, is dramatically reduced in GMH1 depletion (Fig. IV.14). Thus, GEF1 may recruit GMH1 to the endo-lysosomal pathway because the deletion of an 
essential protease PEP4 into gmhl mutants exhibits strong intensity of GEF1-GFP compared to the single gmhl mutant such as Lev-nAChR subunit UNC-29 that came back in a lysosomal formation protein cup-5 deletion in a similar manner (Eimer et al., 2007). How would GMH1 be connected to GEF1? They localize at the Golgi-endosomal interface, however, the localization of GEF1-GFP seems not to be closely affected in GMH1 deletion, although it appears to be unstable positioned as culture conditions in yeast (controversial to be determined by the colocalization microscopy). Thus, GEF1 may be recruited indirectly by GMH1 and still remains ambiguous relevance in the trafficking route that has to be identified with further studies.

\subsection{The ARF-dependent cascade trafficking by UNC-50/GMH1}

Why does UNC-50 exist in eukaryotic cells? Previous studies showed that UNCL, the mammalian orthologous of UNC-50, is required in development, differentiation and maintaining of periodontal ligament fibroblasts and, found as an inner nuclear membrane protein (Fitzgerald et al., 2000; Kim et al., 2007). Alternatively, on the basis of the trafficking of Lev-nAChRs in C. elegans and the ARF-GEFs binding partner as a transmembrane receptor in yeast (Chantalat et al., 2003; Eimer et al., 2007), UNC-50 may be routinely recruited in the cascade trafficking network to sort cargoes. Also it regulates membrane protein secretion and cycles machinery components by sorting away from TGN into the ARF-GEFs-dependent retrograde route to be discarded or not into lysosomes (Fig. V.2).

As a cascade trafficking model, the ARF-GEFs for the activation of ARF GTPases would occure functionally consecutive events from early-Golgi (GBF/GEA/GNOM subfamily) to the post-Golgi (BIG/SEC7 subfamily) with the activation of the distinct ARF GTPases, respectively. The ARF GTPases can be stably recruited by the consecutive-ARF-GEFs and its HDS C-terminal domains for membrane recruitments to the TGN (Lowery et al., 2013; Richardson et al., 2012). The recent two publications (Lowery et al., 2013; Richardson et al., 2012) support the cascade trafficking model (Fig. V.3). In their results, the large ARF-GEFs GBF1, the mammalian ortholog of GEA1/2, is

present in the entire Golgi (early-medial-TGN) and required for the activation of the 
distinct ARF GTPases. They also showed that the ARF-GTP form was recruited at the membrane through the binding of HDS domains of the SEC7 ARF-GEF and its membrane regulator. This indicates that it is likely to be a physical binding transmembrane receptor such as UNC-50/GMH1.

As such, studying UNC-50/GMH1 involved in ARF-dependent vesicle trafficking could contribute to better understanding how cargoes sort and transport to the destinations via the Golgi-endosomal interface in the secretory pathway. Despite the complexity of multimeric-protein trafficking pathway, the selectivity of Lev-nAChRs has contributed to reveal not only the functional roles of ligand-gated ion channels but also how its intracellular trafficking is regulated by UNC-50-dependent. Our analysis in two organisms establishes a role as a trafficking regulator as important features of UNC-50 in eukaryotic cells. Therefore this study will enable a means to further decipher the mechanism of intracellular trafficking and provide clues to develop therapeutic strategies in neuro-pathological disorders. 
A

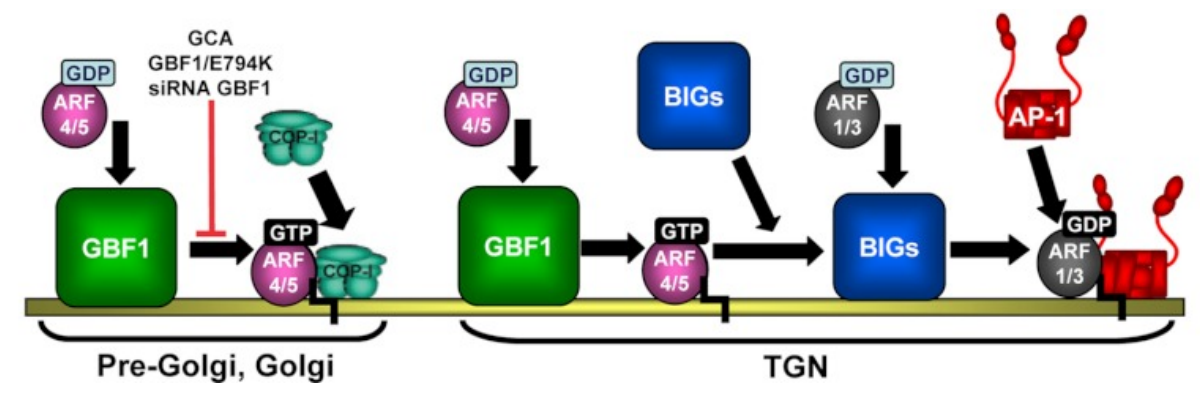

B

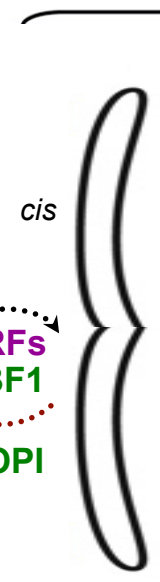

UNC-50/GMH1
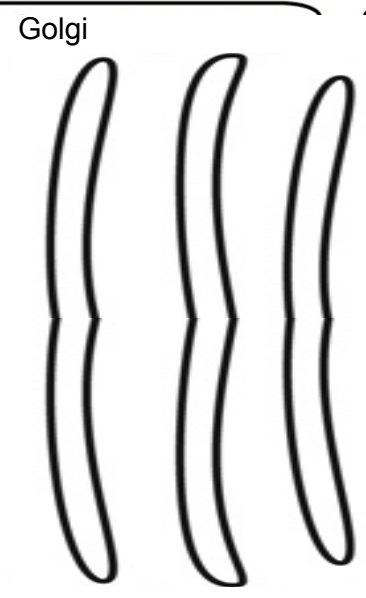

UNC-50/GMH1
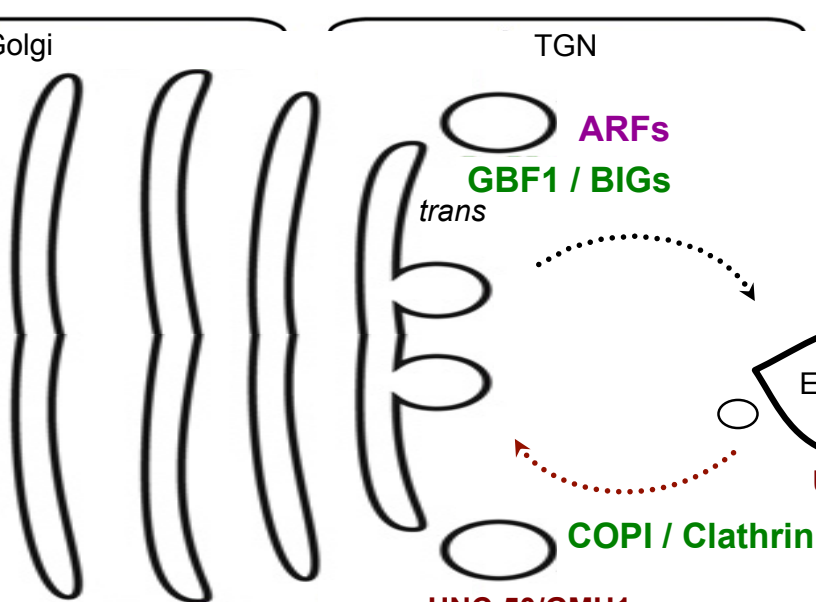

UNC-50/GMH1

\section{C}

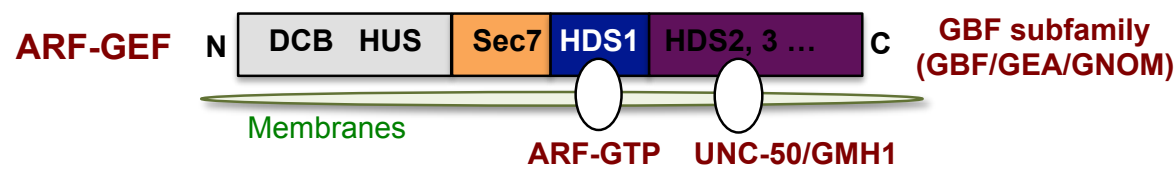

Figure V.3 The ARF-dependent cascade transport manners by UNC-50/GMH1. The functional cascade ARF-GEFs from early-Golgi to TGN represent in secretory pathway that GBF1 recruits ARFs-GTP in the early-Golgi and the ARF4/5 activated by GBF1 recruits again the distinct ARF-GEFs BIG1/2 to the TGN that mediates to the clathrin-adaptor complexes by the activation of ARF1/3 recruited by BIG1/2 of the TGN. The early acting ARF-GEFs may play a key regulator of the coated vesicle transport in secretory pathway (A). The early acting ARFGEFs affect in the TGN with the distinct ARFs but in the same manner (B). The membrane recruitments of the ARFs-GTP are mediated by the GBF subfamily ARF-GEFs (GBF/GEA/GNOM) through the binding of the HDS domains and the transmembrane receptor UNC-50/GMH1 may act as a membrane regulator (C). Source: A was taken from the reference (Lowery et al., 2013) and B and C were modified and depicted from the reference (Richardson et al., 2012). 


\section{CHAPTER II}

Proteomic/Metabolic analysis of the UNC-50 by stable isotope labeling of amino acids in C. elegans 


\section{SPECIFIC INTRODUCTION}

To compare changes in protein levels or modifications between mutants or different environmental conditions, quantitative proteomics is used in combination with mass spectrometry (MS) (Schmollinger et al., 2012). In order to compare quantitative proteomes between two samples, one of them needs to be labeled. Metabolic labeling in vivo brings minimized variations, clear incorporation and no side reactions between two cell populations that are mixed equally during sample preparations. In contrast to the labeling in the metabolic process, samples of chemical labeling are isolated after the metabolic process that remains incomplete labeling of the sample and potential quantitation errors of the separate sample treatments in two different or multiplex samples (Craft et al., 2013; Mann, 2006).

\subsection{Stable isotope labeling of amino acids in living cells (SILAC)}

Metabolic stable-isotope labeling is limited in living cells. Labeling of heavy stable isotope in carbon $\left({ }^{13} \mathrm{C}\right)$ and/or nitrogen $\left({ }^{15} \mathrm{~N}\right)$ and stable isotope labeling by amino acids in cell lines, termed as SILAC, have been widely applied for isotopic labeling to incorporate into biological samples comprising microorganisms (e.g., bacteria and yeast), plants, mammalian cells, mice, fruit flies and nematode C. elegans (Findlay et al., 2008; Fredens et al., 2011; Gruhler et al., 2005; Krijgsveld et al., 2003; Kruger et al., 2008; Lahm and Langen, 2000; Larance et al., 2011; Oda et al., 1999; Ong et al., 2002).

In order to incorporate heavy isotope-labeled atoms (e.g., ${ }^{2} \mathrm{H},{ }^{13} \mathrm{C},{ }^{15} \mathrm{~N}$ or ${ }^{18} \mathrm{O}$ ) of amino acids into proteomes by SILAC, the minimal media including heavy-labeled essential amino acids (e.g., arginine and/or lysine) is supplied to the cell and organisms in which the light/natural-isotopes can be substituted by labeling heavy-nitrogen and/or heavycarbon in essential amino acids. Due to the incorporation of 'heavy' amino acids, the proteins digested into the peptides after trypsin digestion are shifted to higher masses in the mass spectrometry. If the 'heavy' protein extract is mixed with a metabolically unlabeled control extract at equal amounts, the peptides can be unambiguously identified and quantitatively compared. (Ong and Mann, 2006). For example, labeling the amino 
acid lysine- ${ }^{13} \mathrm{C}_{6}$ as a form of heavy-isotope can be metabolic incorporated into proteomes in vivo through the intrinsic cycling of protein synthesis and turnover in cellular growth during culture in the treated/feeding media. In the results, it appears a 6-Da mass shift of the lysine- ${ }^{13} \mathrm{C}_{6}$ from the light/natural-isotope (lysine- ${ }^{12} \mathrm{C}_{6}$ ) labeled samples and differentiates relative protein quantification by measuring peak ratios through MS analysis (Fig. VI.1) (Ong and Mann, 2006).

In addition to the typical SILAC whole proteome analysis, it can be used as internal standards in both relative and absolute quantification. For using a standard, non-labeled or soluble-tagged cultured cells to be purified in multiple conditions are separately combined with the SILAC standard. In the process, SILAC standards can be stable isotope-labeled as well as measurement of absolute amount to be divided by the relative ratio of the other sample to the standard (Deeb et al., 2012; Geiger et al., 2010; Geiger et al., 2011; Zeiler et al., 2012).

With the accurate quantification by SILAC labeling, the affinity purifications of protein complexes using the endogenous/exogenous tags of a specific protein of interest (POI) allow a powerful platform combined with MS. The tagging of POI is to identify relativeinteracting proteins and protein-protein interactions including direct, indirect and lowaffinity interactions in which non-specific contaminants can also be distinguished in representing equal abundances in both control and experiment samples (Emmott and Goodfellow, 2014; Emmott et al., 2013; Mittler et al., 2009; Rhee et al., 2013).

In order to identify specific protein interaction partners with quantitative approaches, the combination of SILAC labeling with affinity pull-downs of a protein of interest follows principally experimental procedures in order: i) isotope-labeling in metabolic conditions and tagging of an interesting-protein by using GFP, FLAG, biotin and primary antibodies (Dobreva et al., 2008; Mittler et al., 2009; Rothbauer et al., 2008; Selbach and Mann, 2006; Trinkle-Mulcahy et al., 2008), ii) co-immunoprecipitations with affinity bead-matrices against tags of proteins, iii) purifying protein complexes of either endogenous or tagged fusion proteins, iv) equally mixes of eluted protein complexes, v) digesting peptides with trypsin and vi) identifying interaction proteins with quantitative ratios of isotopic heavy- and light-labeled peptides (Dobreva et al., 2008; Emmott and Goodfellow, 2014; Mittler et al., 2009; Rothbauer et al., 2008; Schmollinger et al., 2012; 
Selbach and Mann, 2006; Trinkle-Mulcahy et al., 2008). In the process, stable isotope labeling strategies and particular protein-tags can be modified in accordance with various cellular/biological processes and validated in reverse isotope labeling in replicated experiments about targeted molecules, sample treatments and genetic conditions, which provides accurate identification and quantification of proteomes in living cells and organisms (Gouw et al., 2010; Gouw et al., 2011; Krijgsveld et al., 2003; Mann, 2006; Ong et al., 2002).

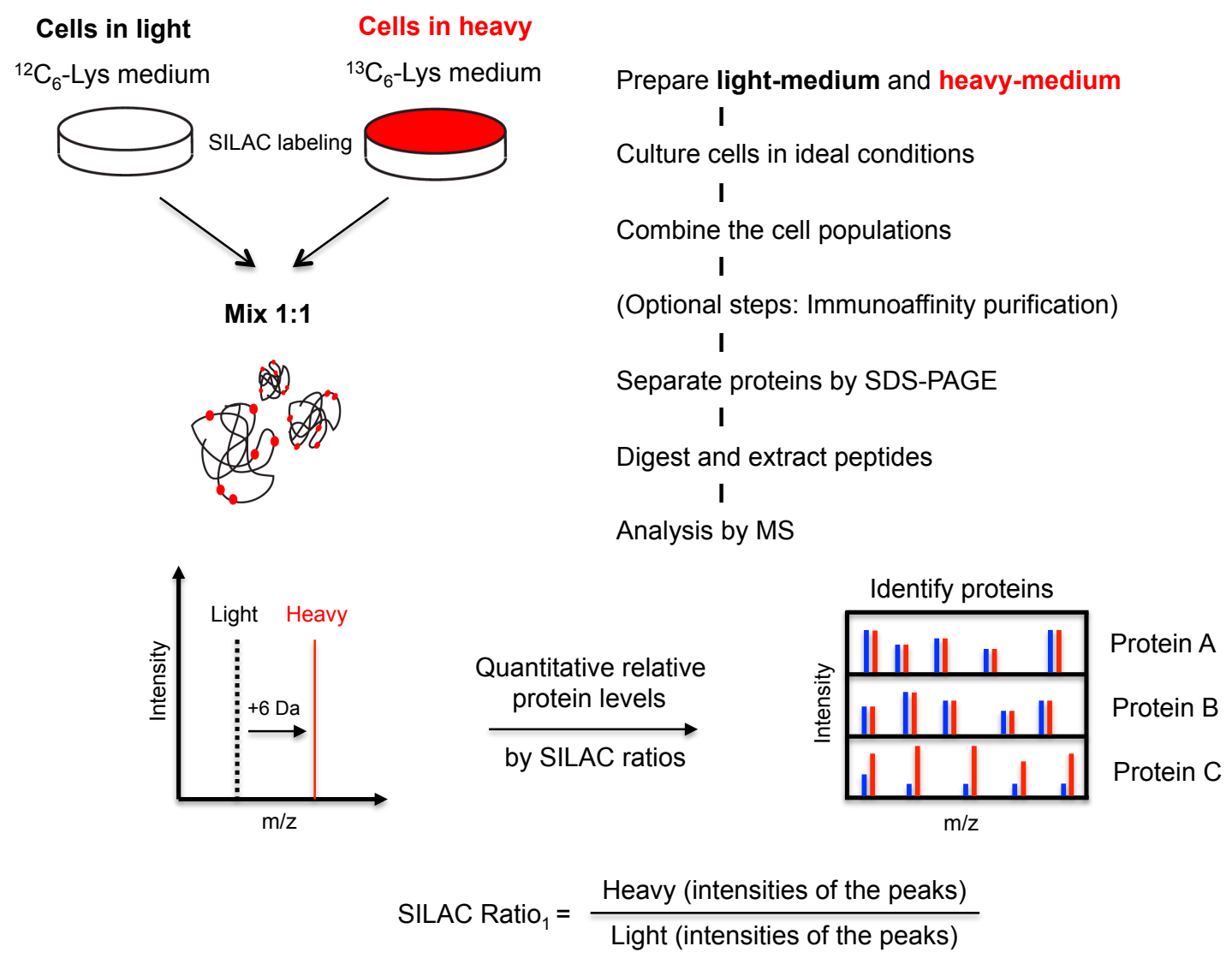

Figure VI.1 Schematic workflow of stable isotope labeling by amino acids in living cells (SILAC). Stable isotope labeling in living cells is applied for accurate quantification of proteins in two or multiple populations eluted in one-mixed samples prior to the MS analysis. For the SILAC labeling, the isotopes heavy and light forms of amino acids are supplied in SILAC media which contain essential amino acids to be incorporated into proteomes through in vivo occurring turnover of proteins. Equal amounts of protein extracts are mixed and, if applicable, immunoaffinity purification of tagged fusion proteins of interest using proper binders covalently coupled to beads at an equal concentration are subjected to eliminate non-specific contaminants 
before separation steps on SDS-PAGE and the proteins digested with trypsin are fragmented to isotopic peptides. Thus, differentially labeled peptides are quantified in SILAC ratios and specific proteins can be represented by fold changes of ratios in certain conditions in which non-specific or environmental contaminants (Protein A and Protein B) in quantitative abundances are close to 1:1 ratios. The peptides of Protein $\mathrm{C}$ can be considered as a specific interaction partner in abundance of relative levels by SILAC ratios. Source: Picture was modified and taken from the references (Geiger et al., 2011; Oda et al., 1999; Ong and Mann, 2006).

\subsection{BioID to identify interacting proteins by Biotin protein ligase (BirA)}

Physical binding proteins in vivo of a protein of interest (POI) are generally identified by high-affinity protein capture techniques to be applied for mass spectrometry (MS)-based proteomics. In order to detect low affinity or very dynamic interaction that can not be stable purified by co-immunopurification experiments such as protein interactions of membrane trafficking events, a high-affinity method is necessary for identification of proteins. As a unique and stable labeling used in living cells, BioID is based on a proximity-dependent protein identification method by using biotin protein ligase (BirA), by which interaction proteins of POI can be targeted as proximal biotinylated proteins in living cells (Choi-Rhee et al., 2004; Roux et al., 2013).

BirA $(35.5 \mathrm{kDa})$ is the Escherichia coli biotin ligase that regulates biosynthetic biotin transcription as an inducible repressor and catalyzes the biotinylation of POI by covalently attaching biotin to a specific target lysine residue of carboxylase substrates (Chapman-Smith and Cronan, 1999). In the process, biotin molecules are activated by ATP-dependent manners to form a biotinyl-5'-AMP (bioAMP) as a reactive intermediate which is tightly bound to the BirA active sites and directly transferred to the $\varepsilon$-amino group of the specific lysine of the protein (Fig. VI.2A) (Chapman-Smith and Cronan, 1999; Roux et al., 2012).

To label binding partners of a given POI, the protein is expressed as a fusion protein with BirA. Since BirA only transfer biotin to specific target protein, the BirA-tag has to be modified for efficient proximity in vivo. A mutated residue of BirA (called BirA*_R118G, highly promiscuous form) is used instead that has a reduced capacity to hold on the activated AMP-biotin intermediate which is able to diffuse out of the BirA* 
enzyme and react with proteins in close proximity. Based on the promiscuous reaction, the BirA* is usefully applied in various cell types for identification of promiscuously neighboring protein interactions to avoid in both unspecific DNA binding and selfassociation as well (Kwon and Beckett, 2000; Roux et al., 2013; Roux et al., 2012). Thus, BirA*-fusion protein catalyzing the reactive bioAMP between the carboxyl group of biotin and ATP leads to promiscuously be biotinylated in interacting proteins of POI that can be eluted by high-affinity biotin-streptavidin purification methods prior to MS analysis (Fig. VI.2B). The fusion protein of BirA* can also be endogenously expressed in living cells containing the supplement biotin.

A
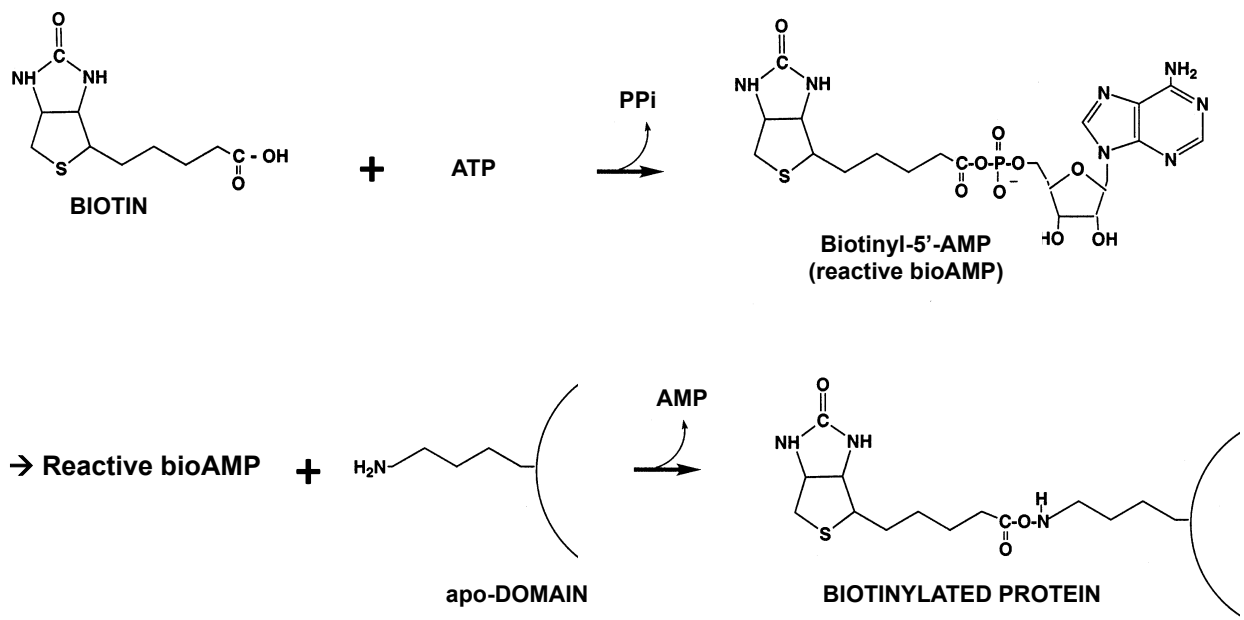

B

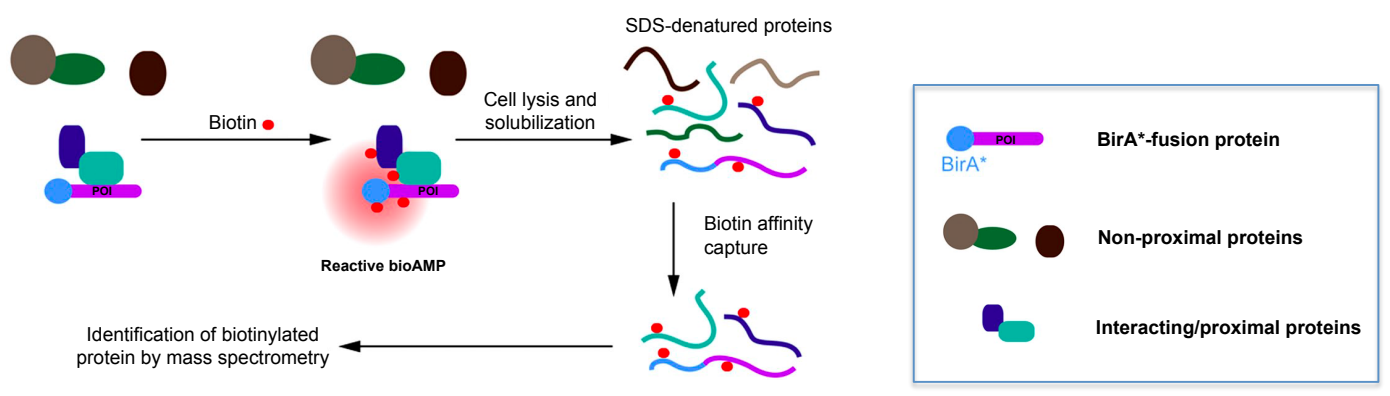

Figure VI.2 Schematic representation of the enzymatic protein in vivo biotinylation mechanisms of the biotin ligase reaction. Biotin (vitamin $\mathrm{H}$ ) is an essential organic cofactor known as covalently attached to several metabolic enzymes carboxylases, decarboxylases and transcarboxylases and active when the protein is coupled in biological processes involved in carbohydrate metabolisms found in plants, fungi and bacteria. Biotinylation of proteins, a rare 
modification in nature, is catalyzed by the enzyme substrate BirA in which an oxygen atom of carboxyl group of biotin and the alpha phosphate $(\mathrm{P} \alpha)$ of ATP are formed to the Biotinyl-5'-AMP (bioAMP) plus pyrophosphate (PPi). The reactive bioAMP is then attached to a primary amine $\left(\mathrm{NH}_{3}{ }^{+}\right)$of lysine residues of the biotin acceptor domain of protein substrates. Once the linkage between the amide group and the bioAMP is formed it sustainably remains the attached state in life of the proteins (A). As a highly promiscuous form, BirA* (R118G) is used to identify proximal/interacting proteins of POI. The BirA*-fusion protein of POI is formed to the reactive bioAMP as promiscuously biotinylation proteins with interacting proteins in vivo reaction and the putative binding partners of POI can be purified by biotin affinity captures of streptavidin coated beads $\left(\sim 10^{-14} \mathrm{M}\right.$ of $\mathrm{K}_{\mathrm{d}}$, dissociation constant) and identified by mass spectrometry (B). Source: A and $\mathrm{B}$ were taken and modified from the references in order (Chapman-Smith and Cronan, 1999; Roux et al., 2012).

\section{3 $\alpha$ GFP-nanobodies for functional studies in living cells}

Direct protein tagging and modifications in vivo are difficult to execute since proteins, especially large complexes or attached/embedded in membranes, might not accept every fusion tag. GFP has a tractable probe as a good fusion tag allowing the tracking and localization of proteins by fluorescence microscopy. Thus GFP fusion proteins have been widely used for protein labeling and large numbers of GFP-fusion constructs are directly available. In addition, by using anti-GFP nanobodies-GFP fusion the complexe can be tagged further studies in vivo (Chakravarty et al., 2014; De Meyer et al., 2014; Kubala et al., 2010). As functional approaches to manipulate and verify proteins in cellular processes, biological nano-complexes of the GFP-nanobody in studying the intracellular localization, binding dynamics and affinity isolation of proteins have been developed to be applied in living cells (Kirchhofer et al., 2010; Rothbauer et al., 2008; Rothbauer et al., 2006; Schornack et al., 2009) and also efficiently used in immunological applications (Groot et al., 2006; Jobling et al., 2003; van Koningsbruggen et al., 2003). In preclinical purposes, the molecular imaging studies in human cancers using various imaging techniques requiring radiolabeled nanobodies as proves have also been extensively investigated (Chakravarty et al., 2014). 
Origin of the nanobodies came from the Camelidae family that possesses unique heavy chain-only antibodies containing a single variable domain antibody fragment $\left(\mathrm{V}_{\mathrm{H}} \mathrm{H}\right.$, variable domain of heavy chain antibody) unlike typical antibodies that consist of heavy and light chains (Fig. VI.3) (Hamers-Casterman et al., 1993; Muyldermans et al., 2009).

The GFP-binding antibody or GFP-nanobody ( $\alpha$ GFPNB $\left.\mathrm{V}_{\mathrm{H}} \mathrm{H}, 12-15 \mathrm{kDa}\right)$ indicating high affinity and specificity binding of the target fluorescent proteins (GFP and some variants) is a highly soluble, smallest size (2.5 $\mathrm{nm}$ in diameter and $4.2 \mathrm{~nm}$ in length) naturally derived and stable protein fragment that contains three hypervariable loops called as complementarity determining regions (CDRs) to be genetically altered as a primary antigen-specific interacting region for the fluorescent proteins (Fig. VI.3) (Chakravarty et al., 2014; Kubala et al., 2010; Rothbauer et al., 2008; Rothbauer et al., 2006).

As crucial advantages in living cells, the GFP-nanobodies can be fused with cellular proteins of interest to trace GFP fusion proteins with their interacting proteins to be detected in biochemical and proteomic analyses as endogenous antigenic epitopes in vivo that provide multifaceted information based on microscopic, biochemical and functional aspects about cellular functions, structures and processes (Rothbauer et al., 2008; Rothbauer et al., 2006). The $\alpha$ GFP nanobody ( $\alpha$ GFPNB) may also be combined with the BioID technology by using a $\alpha$ GFPNB-BirA* fusion to attach the BirA* tag to GFPfusion protein to label their binding partners in vivo. 
A

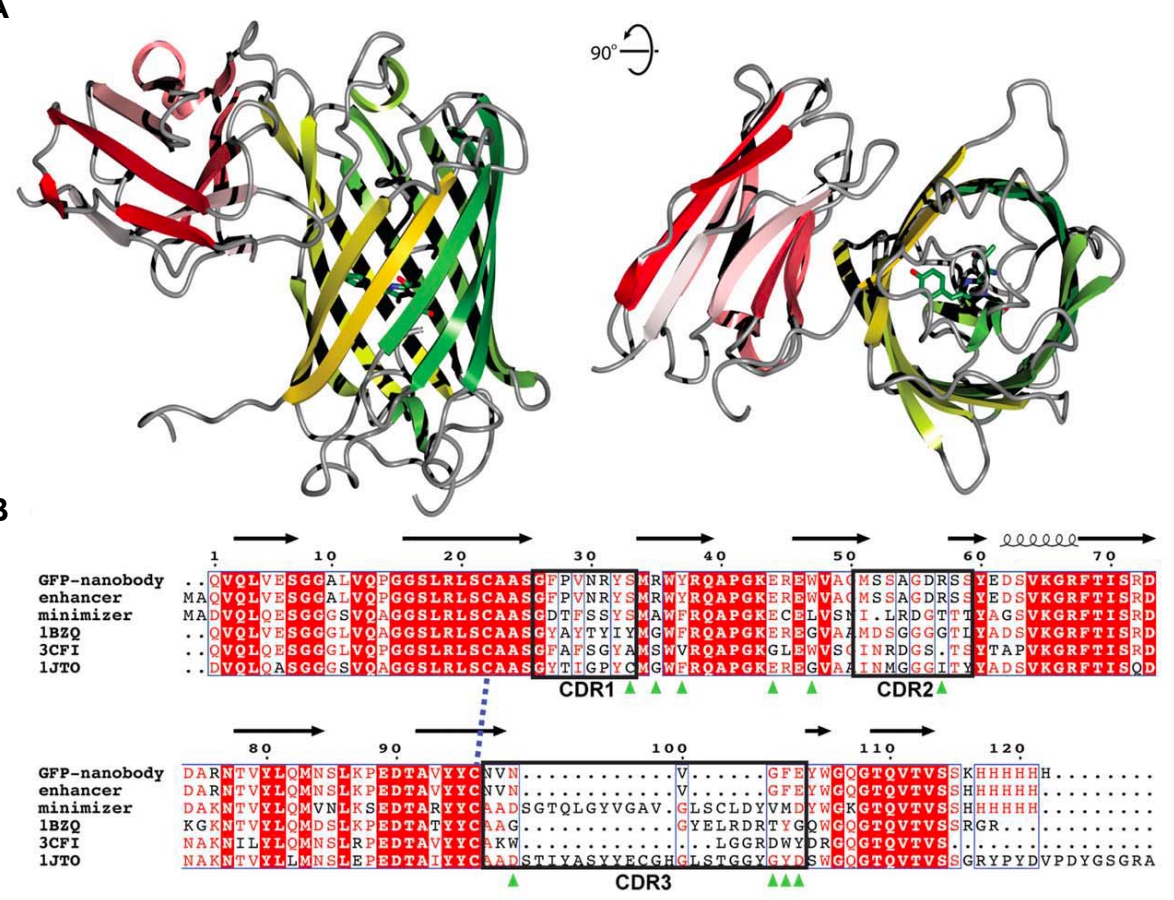

C

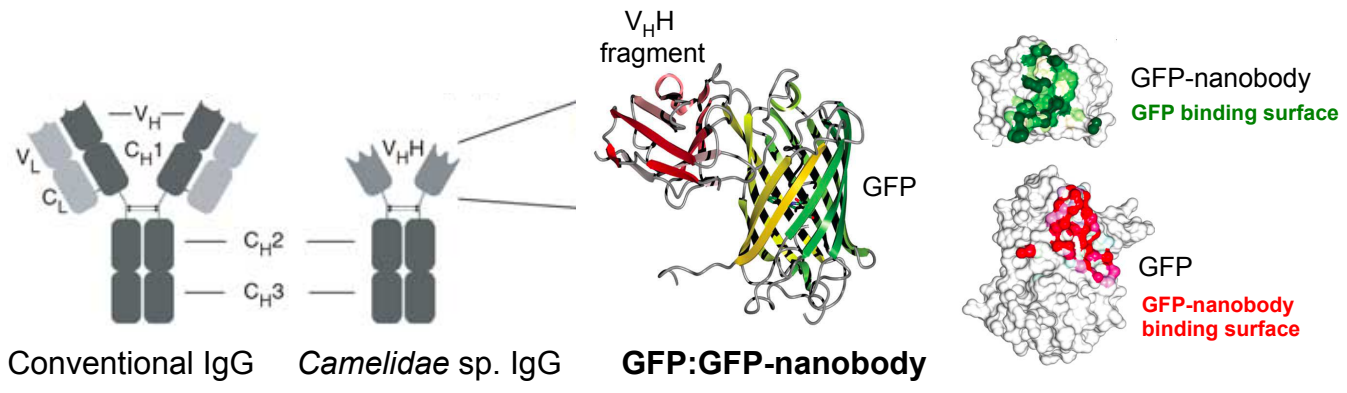

Figure VI.3 The complex structure of the antigen-GFP with the anti-GFP-nanobody fragments. The heterodimeric complex of GFP:GFP-nanobody showing in the asymmetric unit by X-ray crystallography (GFP in green-gold and GFP-nanobody in pink-red) (A). Alignment of the GFP-nanobody sequences with the known camelid-antibody fragments. Three CDR regions are indicated as boxed and a disulfide bridge is marked as a dashed line. Physical GFP-binding sites of the nanobody are shown as green arrowheads in which CDR3 is indicated as major contact points for GFPs. The fragments of the GFP-nanobodies are composed of nine antiparallel $\beta$-strands configuring two sandwiching $\beta$-sheets (B). Schematic representations of conventional structures of typical antibodies and camelid nanobodies with the binding surface shown by cutting-away approach between GFP and GFP-nanobody (C). Source: A and B were taken the reference (Kubala et al., 2010) and C was taken and modified from the references (Kubala et al., 2010; Rothbauer et al., 2006). 


\subsection{Studying proteome in C. elegans}

The nematode $C$. elegans as a simple model organism to understand higher eukaryotic systems is about $60-80 \%$ homologous to humans in genomes and highly classical aspects like mammalians in organization and function of intracellular organelles, biosynthetic pathways and molecular mechanisms (Hulme and Whitesides, 2011; Kaletta and Hengartner, 2006).

In the metabolic processes, biosynthesis and breakdown of dietary sources, needs of essential/non-essential amino acids and energy storage as lipid-formed, are substantially similar with humans (Table VI.1) (Braeckman et al., 2009; Szewczyk et al., 2003). Its dietary source contains proteins and cofactor molecules required for fundamental pathways in cellular respirations such as $\beta$-oxidation of fatty acids to generate acetyl-coA that goes to the electron transport chain and ATP synthesis (Hulme and Whitesides, 2011; Szewczyk et al., 2003). Moreover, the proteomes related to the neurobiological mechanisms for synaptic transmission in biosynthesis, secretion and elimination of neurotransmitters are highly conserved between humans and C. elegans (Table VI.1) (de Bono and Maricq, 2005; Husson et al., 2007). As such, studying proteomes of C. elegans based on the metabolic processes can be powerful tools to understand function and mechanisms of cellular processes in eukaryotic systems.

In terms of studying proteome in C. elegans, MS-based quantification methods with isotope $\left({ }^{15} \mathrm{~N}\right)$-labeled and/or SILAC labeling into proteomes in the metabolic stages via feeding bacteria have allowed accurate quantitative identification of relative proteomes in C. elegans (Fredens et al., 2011; Krijgsveld et al., 2003; Larance et al., 2011). To complete metabolic labeling into proteomes by SILAC, C. elegans is a suitable model organism that requires essential amino acids like humans, in which protein complexes synthesized by metabolic processes are labeled by feeding nutrients containing the essential amino acids such as lysine and arginine during the growth in the SILAC plates (Fredens et al., 2011; Larance et al., 2011). Furthermore, isolation techniques of a protein of interest using high-affinity purifications combined with SILAC labeling in C. elegans can be spotlighted in versatile pathways for studying proteomes based on functional model organisms. 


\begin{tabular}{|c|c|c|}
\hline Amino acids & C. elegans & Humans \\
\hline arginine & essential & essential in development \\
\hline histidine & essential & essential \\
\hline lysine & essential & essential \\
\hline aspartic acid & non-essential & non-essential \\
\hline glutamic acid & non-essential & non-essential \\
\hline serine & non-essential & non-essential \\
\hline threonine & essential & essential \\
\hline asparagine & non-essential & non-essential \\
\hline glutamine & non-essential & non-essential \\
\hline alanine & non-essential & non-essential \\
\hline isoleucine & essential & essential \\
\hline leucine & essential & essential \\
\hline methionine & essential & essential \\
\hline phenylalanine & essential & essential \\
\hline tryptophan & essential & essential \\
\hline tyrosine & non-essential & essential in development \\
\hline valine & essential & essential \\
\hline cysteine & non-essential & $\mathrm{E}$ in development \\
\hline glycine & non-essential & non-essential \\
\hline proline & non-essential & non-essential \\
\hline Neurotransmitters & Used in C. elegans? & Used in Humans? \\
\hline acetylcholine & Yes & Yes \\
\hline GABA & Yes & Yes \\
\hline nitric oxide & Yes & Yes \\
\hline serotonin & Yes & Yes \\
\hline dopamine & Yes & Yes \\
\hline glutamic acid & Yes & Yes \\
\hline octapamine & Yes & No \\
\hline tyramine & Yes & No \\
\hline epinephrine & No & Yes \\
\hline norepinephrine & No & Yes \\
\hline histamine & No & Yes \\
\hline neuropeptides (different types) & Yes & Yes \\
\hline
\end{tabular}

Table VI.1 Highly conserved essential amino acids and neurotransmitters between $C$.

elegans and humans. Source: It was taken from the reference (Hulme and Whitesides, 2011). 


\subsection{Aims of this study}

Intracellular trafficking in eukaryotic systems is orchestrated by protein dynamics that occurs in protein-protein interactions (PPIs), in which the protein assembled trafficking factors such as cargo-machinery complexes are fundamentally regulated by PPIs on the particular transport pathways that can be identified in quantitative protein levels by mass spectrometry (MS)-based proteomics (Helsens et al., 2011). The conserved transmembrane protein UNC-50/GMH1, a kind of ARF-GEFs binding proteins, undergoes highly dynamic and very transient things in subcellular compartments. As shown in Chapter I, UNC-50 acts as a recycling regulator that is required for endolysosomal traffic flows and function in retrograde pathways of the endosome-to-TGNs.

To identify the related molecules and protein-binding partners on UNC-50-dependent pathways, powerful proteomic technologies were adopted and optimized for accurate quantification and identification of proteomes. In this study, several methods have been accomplished in C. elegans as follows: stable isotope labeling by amino acids (SILAC), proximity-dependent enzymatic fusion protein tags (BioID), tracing anti-GFP nanobodies conjugated with soluble fluorescent proteins in the spatially restricted protein structures and SILAC-combined immuno-affinity purifications (SILAC-BioID).

In Chapter II (Fig. VI.4: STEP7-8), we analyze UNC-50/GMH1 in metabolic stages with minimally disturbed conditions. In order to identify the membrane protein-binding partners that undergo dynamic and transient interacting processes, capture methods of high-affinity protein interactions were used to validate relative quantification and protein identification based on the metabolic labeling by SILAC. From this study, proteomic experiments in C. elegans were optimized to identify quantitative proteins. We found that the anterograde transport promoting proteins ACT-2, NMY-2, GPB-1, ERFA-3, UNC-18 and VPS-32.1 were shown to be quantitative protein abundance. Whereas retrograde transport Rab6-dynein mediated BICD-1 and secretory yolk genes VIT-3 and VIT-5 taken by gonad in the body cavity of $C$. elegans were shown to be down-regulated in unc50 mutants. This is consistent with the results of Chapter I that UNC-50 acts as a trafficking regulator in eukaryotes yeast and C. elegans in which the absence of UNC-50 
leads to enhanced membrane-dynamic flow to the endo-lysosomal/vacuolar compartments that disturbs the recycling of vesicle trafficking machineries in the endosomes-to-TGN.

Additionally the BioID using biotinylated-dependent enzymatic protein conjugation methods was endogenously applied and functionally expressed in vivo with UNC-50 and other soluble proteins GFP and anti-GFP nanobodies in C. elegans. In this study, the advanced proteomic methods were adopted and being established in MS-combined neurogenetic projects that have been done and ongoing experiments in collaboration with the proteomics/metabolomics laboratories. Thus this study will guide as an accurate quantitative and intuitive protein identification approach to be driven in the filed of versatile protein dynamics in order to understand intracellular trafficking mechanisms.

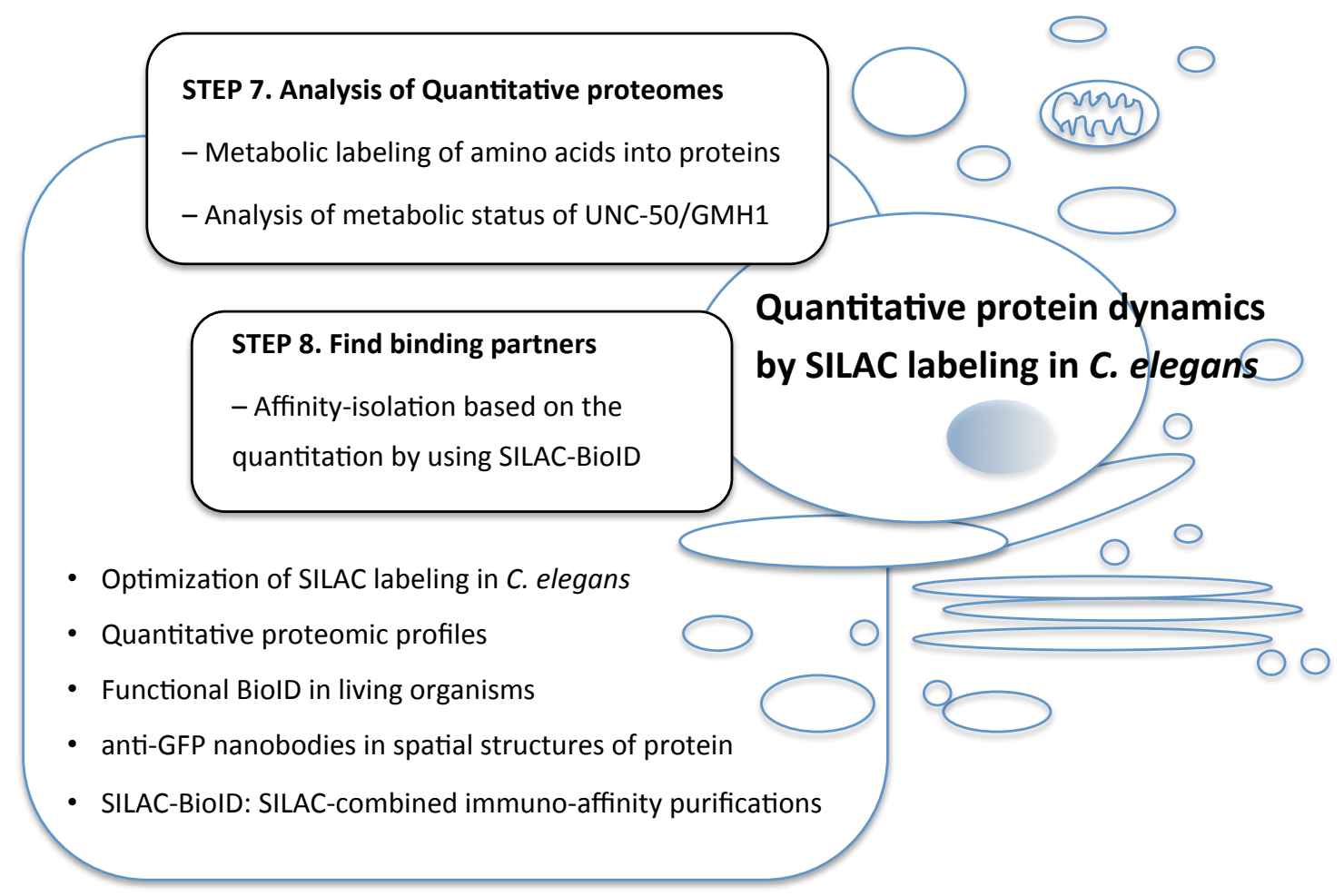

Figure VI.4 Schematic diagrams of research aims for identifying and quantifying protein dynamics on UNC-50/GMH1-dependent in C. elegans. As on-going projects, it consists of parts in proteomics and metabolomics in two model organisms the nematode C. elegans and yeast S. cerevisiae. Major aims of Chapter II are to identify interaction proteins and accurate quantitative protein profiles of the UNC-50-dependent trafficking pathway for understanding the intracellular transport in proteomes. The STEP7 and STEP8 belong to the Chapter II: 
Proteomic/Metabolic analysis of the UNC-50 by stable isotope labeling of amino acids in $C$. elegans. In this study, rising experimental technologies in proteomics were adapted and carried out by the author. After samples optimized in accordance with the research purpose in the model organisms, proteome mass spectrometric data mining were conducted by our collaborators Sebastian Wiese and Carsen Jäger in Core Facility of Proteomics and Metabolomics of ZBSA, Uni-Freiburg and Uni-Ulm. 


\section{RESULTS}

\subsection{SLE1 E. coli strains for SILAC}

Quantitative proteomics in living organisms is gradually applied for which strategic labeling procedures of heavy and/or light amino acids into newly synthesized proteins are facilitated by using the SILAC method that is stable isotope labeling of amino acids in living cells (Gouw et al., 2010; Krijgsveld et al., 2003; Larance et al., 2011; Ong et al., 2002).

In C. elegans, the SILAC can be done by metabolic labeling through feeding worms the E. coli SLE1 strain that is auxotroph for lysine (Lys or K) and arginine (Arg or R). The growth medium then has to be supplied with either light or heavy amino acids. In addition, SLE1 carries the plasmid pAG608 containing the orn-1 targeted RNAi feeding vector to prevent arginine-to-proline (Arg-to-Pro) conversion through the biosynthetic pathway (Fig. VII.1) (Larance et al., 2011).

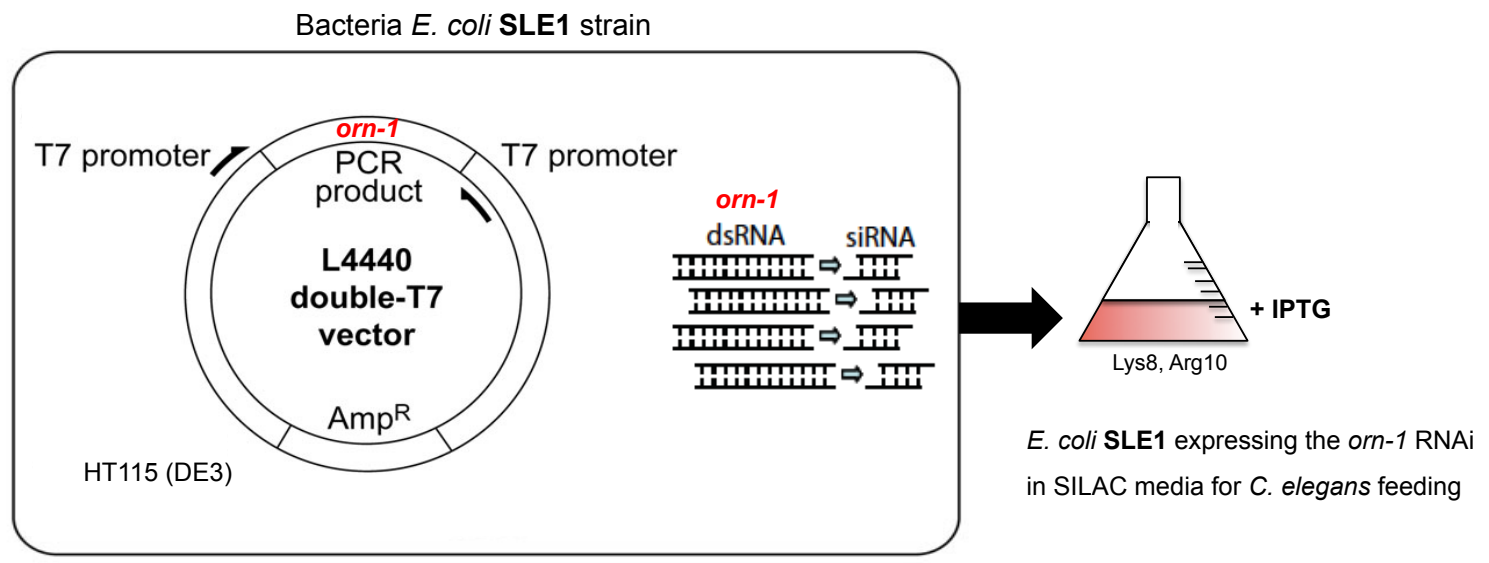

Figure VII.1 Schematic diagram of $E$. coli SLE1 in SILAC media for $C$. elegans feeding. $E$. coli SLE1 driven from the HT115 (lambda DE3) RNase-deficient E. coli strain which is generally used for RNAi via feeding in C. elegans contains the orn-1 targeted RNAi feeding vector and the amino acids arginine and lysine auxotrophy for metabolic labeling by using a given SILAC media. During the growth in SILAC media, $1 \mathrm{mM}$ IPTG is added to induce dsRNA transcription for orn- 1 knockdown targeted by RNAi and the SILAC media are concentrated to be seeded on the nitrogen-free agarose pads. Source: Picture was taken and modified from the pictures of the 
references (Kamath et al., 2001; Larance et al., 2011). SLE1 was made in the group of Lamond and applied in this study as described in the reference (Larance et al., 2011).

To label the amino acids ( $\mathrm{K}$ and $\mathrm{R}$ ) metabolically into the E. coli for C. elegans, the SLE1 E. coli strains were prepared to confirm rates of incorporation of heavy-isotope amino acids into peptides and Arg-to-Pro conversion before feeding worms as described previously (Larance et al., 2011). The SLE1 was streaked out on LB plate from a frozen stock and one colony was grown on the LB containing Amp ${ }^{\mathrm{r}}(100 \mu \mathrm{g} / \mathrm{ml})$ and $\operatorname{Tet}^{\mathrm{r}}(12.5$ $\mu \mathrm{g} / \mathrm{ml})$ plates at $37{ }^{\circ} \mathrm{C}$ for overnight. A single colony was inoculated into M9 minimal medium (M9MM, See the Appendix) containing each $40 \mu \mathrm{g} / \mathrm{ml}(220 \mathrm{nM})$ of light (L-KR; ${ }^{14} \mathrm{~N}_{2^{-}}{ }^{12} \mathrm{C}_{6}$-lysine and ${ }^{14} \mathrm{~N}_{4^{-}}{ }^{12} \mathrm{C}_{6}$-arginine) and heavy (H-KR; ${ }^{15} \mathrm{~N}_{2^{-}}{ }^{13} \mathrm{C}_{6}$-lysine and ${ }^{15} \mathrm{~N}_{4^{-}}$

${ }^{13} \mathrm{C}_{6}$-arginine) amino acids, respectively (Larance et al., 2011).

In order to eliminate the amino acid conversion Arg-to-Pro, the orn-1 targeted RNAifeeding vectors of the SLE1 strains were expressed in the medium M9MM (L-KR and/or $\mathrm{H}-\mathrm{KR}$ ) containing $1 \mathrm{mM}$ isopropyl $\beta$-D-1-thiogalactopyranoside (IPTG) and carbenicillin $(1 \mu \mathrm{g} / \mathrm{ml})$ as a selective marker during the growth until $\mathrm{OD}_{600 \mathrm{~nm}}$ of 1 at $37^{\circ} \mathrm{C}$. Before feeding C. elegans using the E. coli SLE1 for metabolic labeling, the incorporation of the heavy-arginine and lysine into the SLE1 E. coli grown in the media was confirmed to be fully labeled as heavy-isotopic forms and no conversion of the Arg-to-Pro as shown in the previous study that a heavy proline containing peptides does not appear in the bacteria (Larance et al., 2011).

\subsection{Metabolic incorporation rates of the heavy labeled-amino acids in $C$. elegans}

In order to apply SILAC labeling in C. elegans, nitrogen-free conditions except for isotope-labeling supplements in the media and growing plates are necessary for efficient metabolic labeling without surplus nitrogen sources. For this reason, nitrogen-free agarose worm plates (NGM-N) were prepared (Appendix) and the E. coli SLE1 grown in the M9MM L-KR and/or H-KR were concentrated as a 10-fold in $\mathrm{OD}_{600 \mathrm{~nm}}$ of 1 after overnight culture (Gouw et al., 2011; Krijgsveld et al., 2003; Larance et al., 2011). 
To test whether feeding periods with SLE1 bacteria are affected in rates of incorporation of heavy isotopes into C. elegans, different worm generations were used to find proper generations in feeding periods to be incorporated efficiently into the proteomes. For feeding worms on the SLE1 seeded plates which were concentrated as a 10 -fold in $\mathrm{OD}_{600 \mathrm{~nm}}$ of 1 , worms were transferred onto non-seeded NGM-N plates for metabolic labeling with the given amino acids and allowed to lay eggs at $20^{\circ} \mathrm{C}$. All the synchronized worm generations were transferred onto the SLE1-seeded plates (L-KR and/or $\mathrm{H}-\mathrm{KR}$ ) and maintained at $20^{\circ} \mathrm{C}$ on the heavy-amino acids lawn pads (Fig. VII.2A). The worms of F2, F5 and F8 generations in order to confirm metabolic incorporation rates after feeding heavy-isotope amino acids (H-KR) were collected as described previously by flushing the plates in PBS and immediately frozen at liquid nitrogen in cold-PBS solution containing protease inhibitors and stored at $-80^{\circ} \mathrm{C}$ (Larance et al., 2011). The frozen sample was thawed on ice and powdered guanidine- $\mathrm{HCl}$ ( $76 \mathrm{mg}$, bond breakers) and $25 \mathrm{mM}$ Tris (2-carboxyethyl) phosphine hydro-chloride solution (TCEP, reducing agents) were added, vortexed vigorously and heated at $65^{\circ} \mathrm{C}$ for 10 minutes. Before the centrifugation for 10 minutes at $17,000 \mathrm{~g}$ at room temperature, the worm lysates were bead-beated with glass-beads $(0.7 \mathrm{~mm}, 50 \%$ vol. slurry $)$. As an alternative protocol, whole worm lysates frozen with protease inhibitors were boiled at $95^{\circ} \mathrm{C}$ for 10 minutes in SDS loading buffer (Appendix) containing reducing agents such as dithiothreitol (DTT) or TCEP and resolved on SDS-PAGE gels (Appendix, 10\% labmade gels or $4-15 \%$ Mini-PROTEAN ${ }^{\circledR}$ TGX $^{\mathrm{TM}}$ Gel, Bio-rad). The staining protein bands with Coomassie solution (Appendix) after destaining were tryptic digested and analyzed by MS.

As the results, the incorporation rates of F2 have been shown to be $50 \%$ in light (Lys0, Arg0) and heavy (Lys8, Arg10) labeling in peptides and either Arg-to-Pro conversions as red peaks (Fig. VII.2B). The F5 generations as longer-period fed worms on the given heavy-isotope plates were shown to be much higher incorporation of $90 \%$ and less proline conversions shown in black peaks (Fig. VII.2B). Therefore, the feeding periods can not be negligible to be incorporated amino acids into proteins for metabolic incorporations through biosynthetic pathway, even if there are contaminants of some 
natural amino acids from their parents or others that actually can not be possible in the medium nitrogen-free used in this study.

To get complete or higher incorporation of the given amino acids, we found that the feeding periods were not only considered in labeling rates because there was no significant difference between F5 and F8. From these results, the bacteria SLE1 lawn pads were tested whether the concentrations do affect in metabolic incorporation rates via feeding for C. elegans. The concentration of the bacteria can be used a 10-to-50-fold in $\mathrm{OD}_{600 \mathrm{~nm}}$ of 1 on the seeding plates (Gouw et al., 2011; Krijgsveld et al., 2003; Larance et al., 2011). After feeding worms in the 50-fold $\left(\mathrm{OD}_{600 \mathrm{~nm}}\right.$ of 1$)$ seeded plates, the incorporation rates were shown to be improved (Table VII.1) and minimal proline conversions (Sebastian Wiese). These results suggest that the metabolic incorporation rates of the given light or heavy amino acids in the nematode were affected in the treated periods and the concentration of feeding SLE1 bacteria to be incorporated into proteins in vivo. In order to do accurate quantitative studies prior to the protein comparison in certain conditions, the incorporation values of SILAC labeling should be fundamentally concerned in metabolic circumstances of living organisms.

\begin{tabular}{lllll}
\hline Generation of C. elegans wild type & F2 & F5 & F8 & F8 \\
\hline E. coli SLE1 concentration $\left(\mathrm{OD}_{600}\right.$ of 1) & 10-fold & 10-fold & 10-fold & 50 -fold \\
\hline Incorporation (\%) of heavy-isotope (Lys8, Arg10) & 50 & 90 & 90 & 93 \\
\hline
\end{tabular}

Table VII.1 Metabolic incorporation rates by SILAC labeling in generation of $C$. elegans. SLE1 strains are inoculated in $100-500 \mathrm{ml}$ SILAC heavy medium (Lys8, Arg10) and concentrated into $10-50 \mathrm{ml}$ (OD600 $\mathrm{nm}=1,10$-to-50-fold) as a final volume after centrifugation for seeding SLE1 on nitrogen free plates to feed C. elegans. 
A

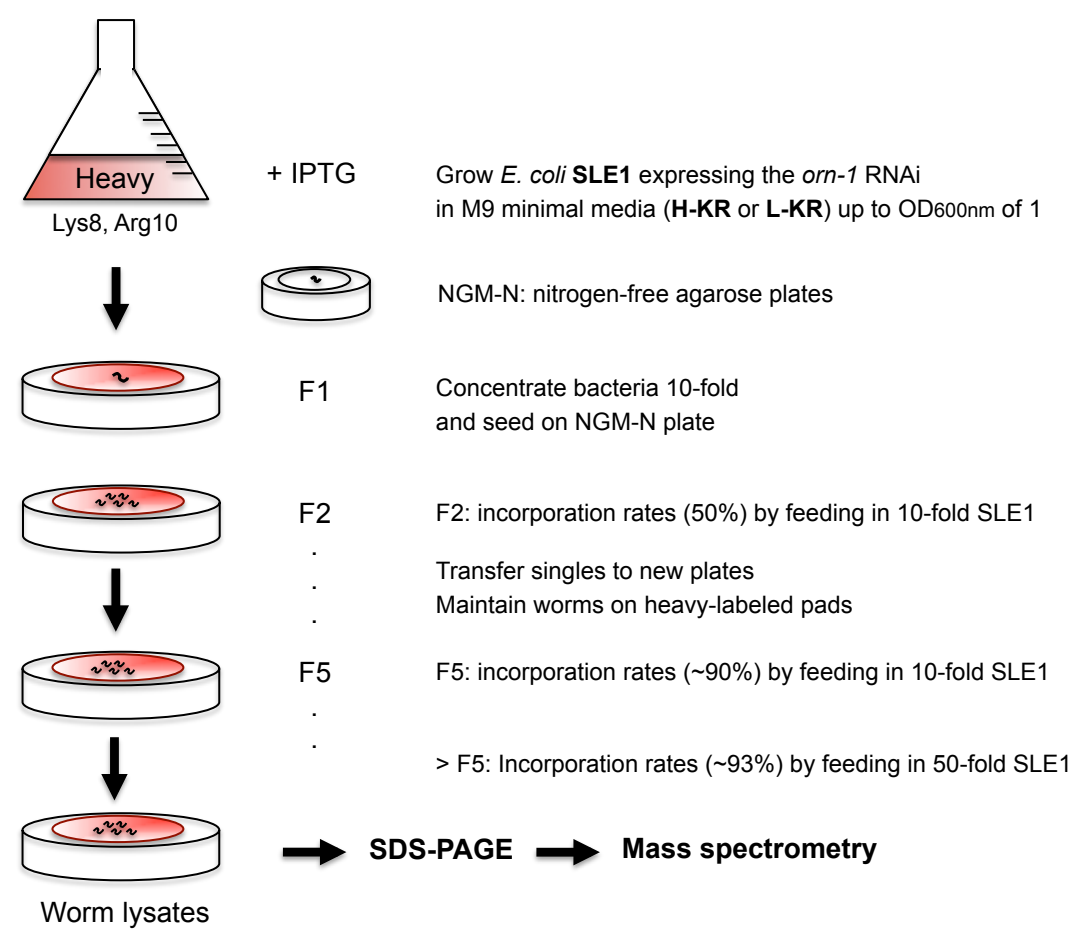

B

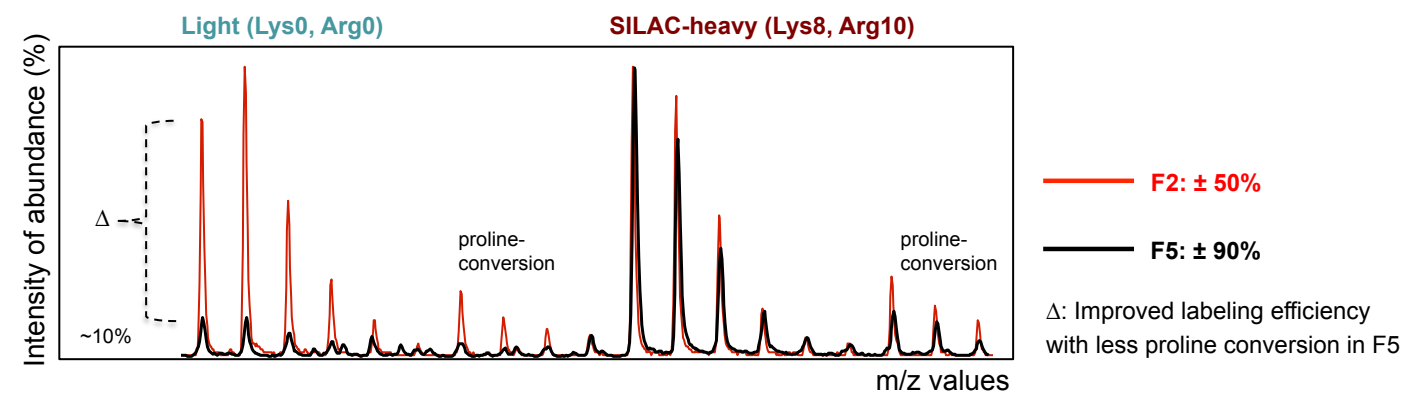

Figure VII.2 Stable isotope labeling by amino acids in feeding periods for C. elegans. Stable isotope labeling by amino acids in C. elegans are shown as workflows: E. coli SLE1 strains containing the orn-1 targeted RNAi feeding vectors are grown in the minimal media with $1 \mathrm{mM}$ IPTG to prevent Arg-to-Pro conversions and seeded on NGM-N plate for feeding worms to be metabolically labeling of amino acids into proteins newly synthesized. Worm lysates treated with protease inhibitors are subjected to resolve on SDS-PAGE, digest proteins with trypsin and detect in mass spectrometry (A). Metabolic incorporation rates of SILAC labeling in C. elegans are affected in prolonged-fed periods and seeded concentrations of the bacteria grown in the given SILAC media. The incorporation rates of F2 (red peaks, 50:50 ratios in the heavy labeling) were much improved to $\sim 90 \%$ and less proline conversions in F5 (black peaks) which was fed in the longer periods on the given heavy-source plates (B). Source: All experiments in SILAC labeling 
in C. elegans were conducted by the author and raw data of peptide abundances by MS shown in B were performed in collaboration with Sebastian Wiese.

\subsection{Analysis of relative quantitative proteomes of unc-50 mutants by SILAC}

Since the SILAC labeling was proven in rates of incorporation in this study, unc-50 mutants were investigated in quantitative proteins compared with the relative abundance profiles of wild type controls. For this purpose, we have referenced two experimental protocols as performed in C. elegans (Fredens et al., 2011; Larance et al., 2011). The light-(Lys0, Arg0) and heavy-(Lys8, Arg10) by SILAC were labeled in wild type controls (WTH, heavy-labeled in wild type and WTL, light-labeled in wild type) and unc-50 (x47) mutant strains (MUL, light-labeled in the mutant) via feeding 50-fold SLE1 bacteria $\left(\mathrm{OD}_{600 \mathrm{~nm}}\right.$ of 1$)$, in which the labeling procedures that have been done previously in metabolic incorporations were used in this study (Fig. VII.3).
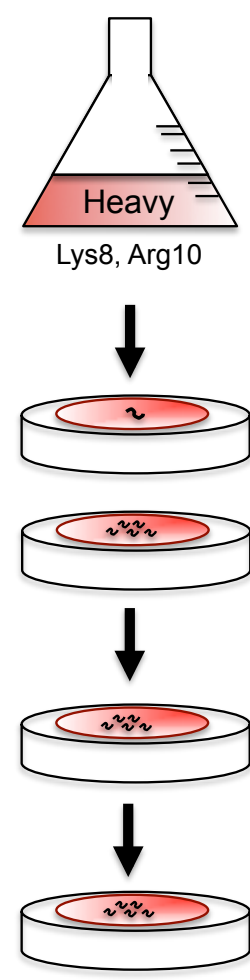

WTH

Wild type

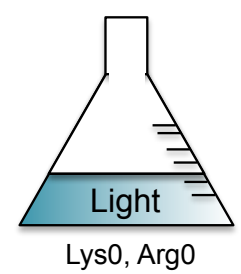

+ IPTG

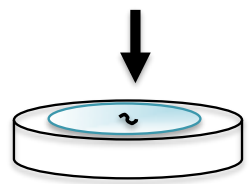

F2
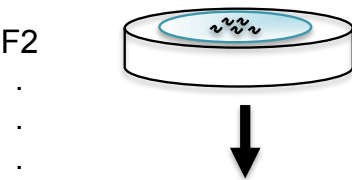

F5

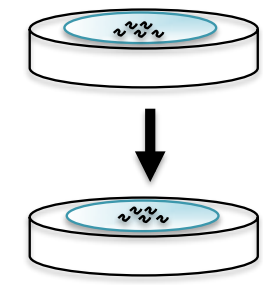

MUL

unc-50 mutants

Mix lysates 1:1

Grow E. coli SLE1 expressing the RNAi in M9 minimal media (H-KR or L-KR) up to OD600nm of 1

Concentrate bacteria a 50-fold (OD600nm = 1) and seed on agarose nitrogen-free plate

Metabolic labeling by SILAC via feeding in 50-fold SLE1

Maintain worms on SLE1 seeded plates

> F5: more than F5 generations to be incorporated

Control SILAC labeling: WTH:WTL (1:1), duplicated Sample SILAC labeling: WTH:MUL (1:1), triplicated

SDS-PAGE and Mass spectrometry 
Figure VII.3 Labeling by SILAC in wild type and unc-50 mutants. To comparison quantitative protein abundances by metabolic labeling, wild types (WT) and mutants (MU, unc50) were labeled as follows: heavy-WT (WTH) and light-WT (WTL) as controls and light-MU (MUL) as samples. The labeling worms in SILAC bacteria plates were mixed equally (1:1) as the control (WTH:WTL) versus sample (WTH:MUL). In order to equal proportions of proteins after the extraction as described previously, a Bradford assay was performed in the supernatant of whole lysates prior to be resolved on 10\% SDS-PAGE gels (Larance et al., 2011).

\subsubsection{Quantification of protein changes in unc-50 mutants by SILAC ratios (H/L)}

As the labeling workflow of the SILAC summarized in Figure VII.3, we assayed quantitative protein abundances in samples driven from whole worm lysates as equal amounts mixed. In this study, the proteins identified were a total of 2307 which was normalized in mean ratios close to one because of, in practice, the experimental values in SILAC ratios heavy:light vary in either WT/WT and/or WT/MU that theoretically should closely be ' 0 ' at the center in the normal distribution. Thus the intensity plots of replicates were normalized shown in both controls and test samples (Fig. VII.4), and then quantification of protein changes was represented on the 2-dimensional scatter-plots of the control replicates $(\mathrm{C} 1$ and $\mathrm{C} 2, \mathrm{WT} / \mathrm{WT})$ and test replicates (T1 and T2, WT/MU) (Fig. VII.5).
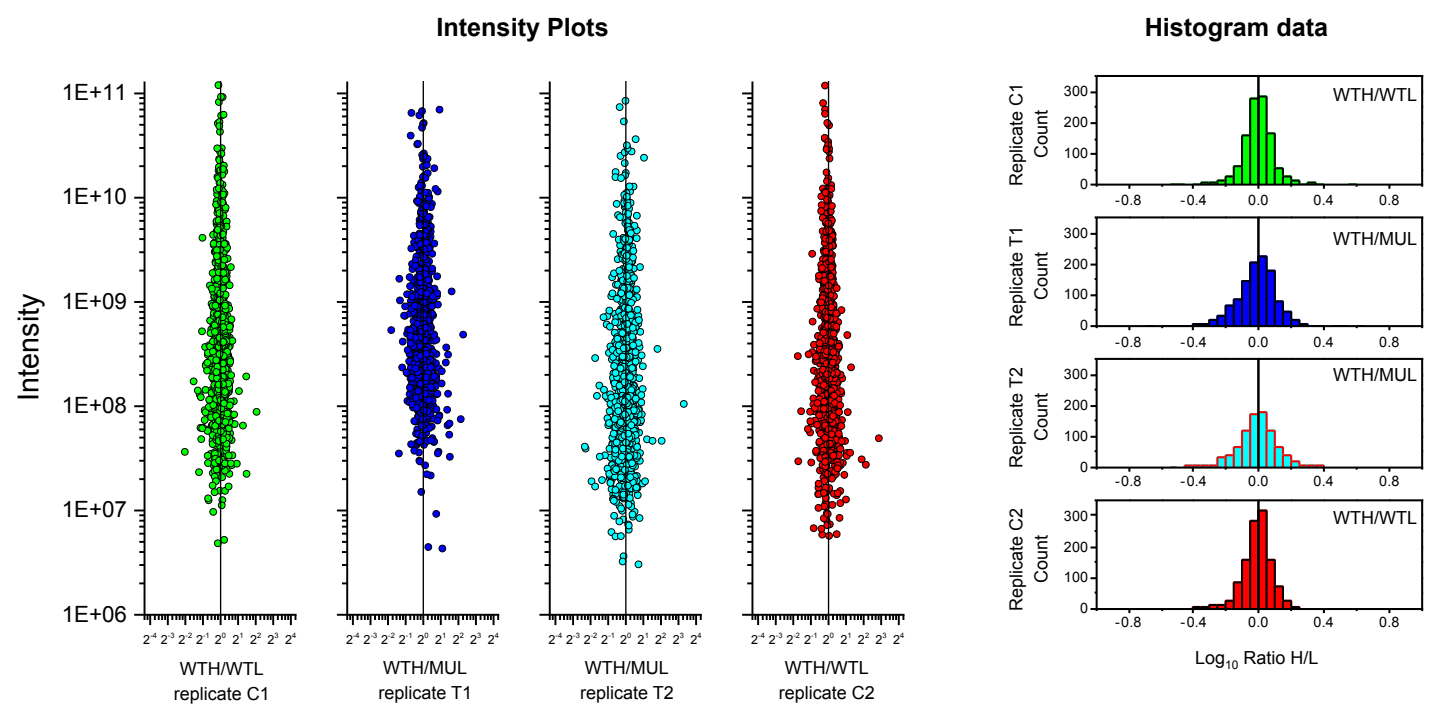

Figure VII.4 Quantitative distribution of the normalized ratios of the SILAC labeling. Experimental replicates in a control (WTH/WTL) and query (WTH/MUL) are shown as intensity 
plots (A) and distribution of histogram data (B) normalized to be centered (0) by SILAC ratios. The control replicates (C1 and $\mathrm{C} 2$ ) and sample replicates (T1 and $\mathrm{T} 2)$ were used in this study. Source: Data were provided from a collaborator, Sebastian Wiese, Laboratory of Proteomics, Uni-Ulm).

A

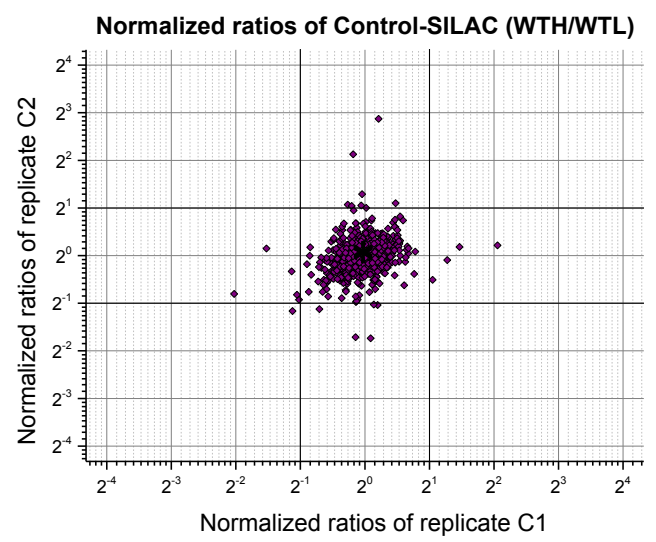

B

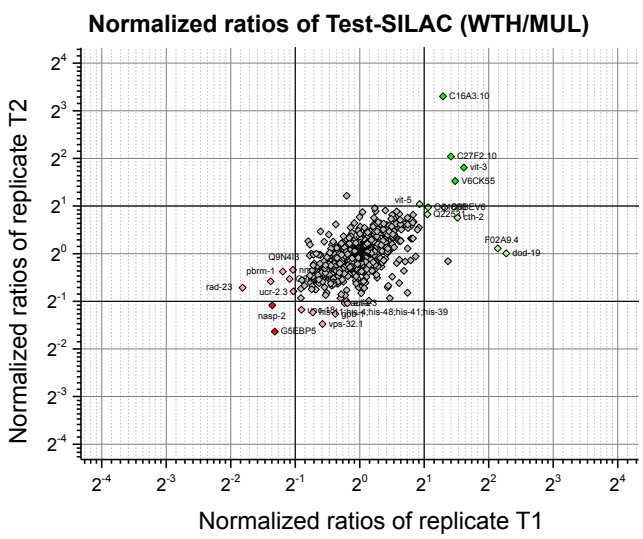

C

Distribution of 2D-Scatter Plots of the merged replicates in normalized ratios of control (C1, C2) and test (T1, T2)

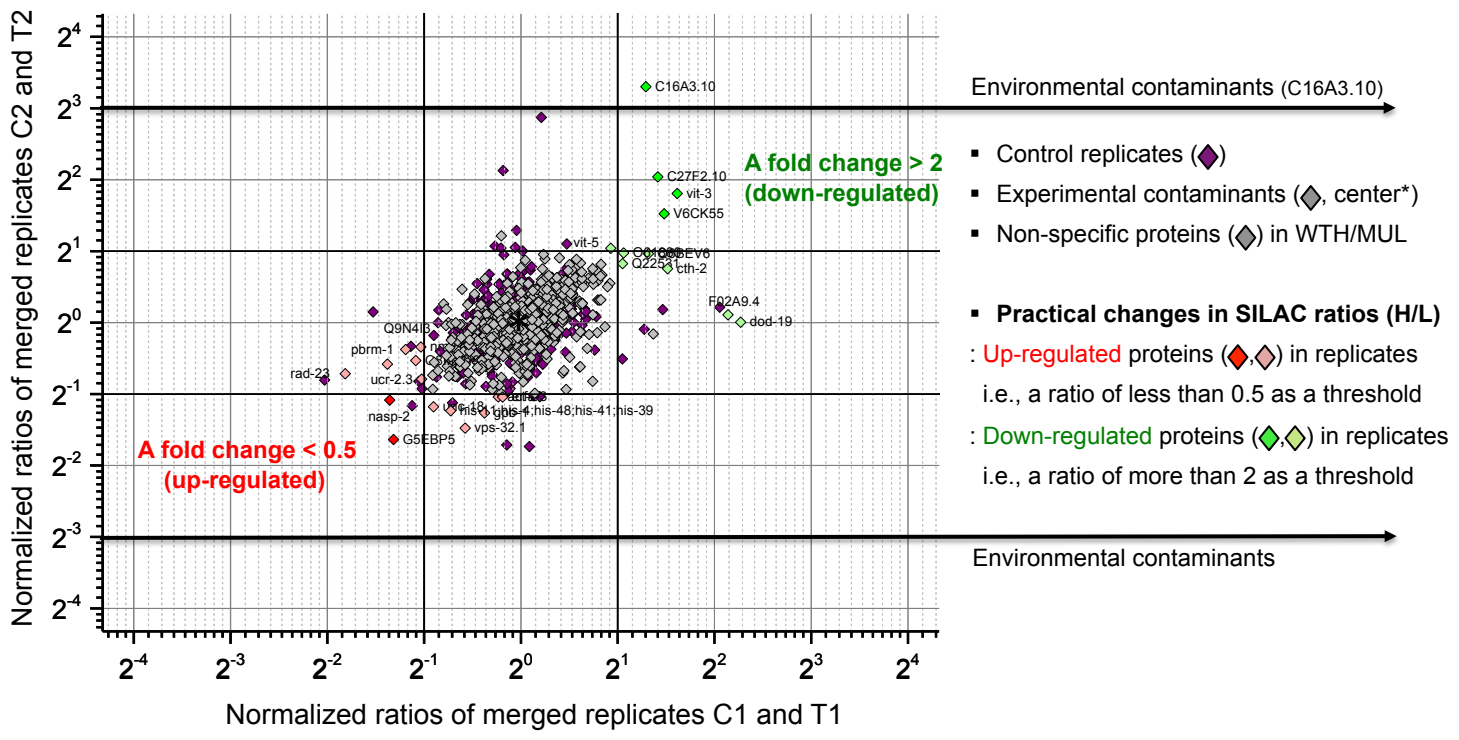

Figure VII.5 Accurate quantification of practical changes of protein identified in unc-50 mutants. Quantitative proteins identified by SILAC labeling H/L are shown in 2D-scatter plots of normalized ratios of control-SILAC (C1 and C2) (A) and test-SILAC (T1 and T2) (B). Data visualized in the plots, clustered at the center that is close to zero marked as an asterisk (*), can be considered as putative experimental contaminants and either external/internal environmental contaminants can be distinguished in SILAC ratios (close to $\sim$ zero) (Boulon et al., 2010). In 
addition to the non-specific contaminants, a few particular proteins based on the experimental conditions such as a targeted-RNAi and its related abnormal expressions should be confirmed in ratios due to probable experimental errors and/or incomplete incorporates. In order to choose a ratio as an arbitrary threshold for the test-SILAC (WTH/MUT), a fold change in SILAC ratios as being significantly regulated were applied to identify specific proteins in unc-50 mutants. The SILAC ratios of less than 0.5 (i.e., a value $<0.5-1$ ) indicate up-regulated proteins whereas a value of ratio more than 2 (i.e., 1 - $2<$ a ratio) means relatively down-regulated proteins against the control-SILAC (C). The 2D-scatter plots merged as all-in-one-SILAC replicates (Control:Test) are visualized in which non-specific and environmental contaminants are distinctly recognized in color plotted (C). From this study, we identified 24 proteins to be changed as a fold ratio except for non-specific and environmental contaminants that include the ornithine aminotransferase of the orn-1 targeted-RNAi feeding vectors used in both SILAC labeling. Upregulated proteins of 14 as a ratio $<0.5$ and down-regulated proteins of 10 as a ratio $>2$ were identified at least in one replicate in the test-SILAC of unc-50 mutants. Source: A and B were provided from the collaborator Sebastian Wiese (Laboratory of Proteomics, Uni-Ulm) and C was annotated by the author in merged scatter-plots of normalized controls and test replicates that all are based on the proteomic results of SILAC labeling conducted by the author.

\subsubsection{SILAC proteome profiling shows trafficking factors are specifically deregulated in $u n c-50$ mutants}

As the results of quantitative protein abundance in unc-50 mutants, we found that protein changes $(0.5 \%$ of total proteins identified) were largely involved in the function of the intracellular transport related and regulatory categories (Figure VII.6).

The proteins shown to be up-regulated as a fold change of SILAC ratio at least in one replicate include NASP-2 (transport of histones) (Nabeel-Shah et al., 2014), ACT-2 (contractile actin filament components), NMY-2 (non-muscle myosin motor proteins), GPB-1 (guanine nucleotide-binding protein subunit beta-1 and anterograde transport promoting factors in early cell divisions) (Thyagarajan et al., 2011), ERFA-3 (Eukaryotic release factor homolog, putative GTP binding and GTPase activity in Gene Ontology), UNC-18 (anterograde promoting transport as a syntaxin-binding protein 1) (McEwen and Kaplan, 2008) and VPS32.1 (vacuolar protein sorting related factors) (Table VII.2A and B). In addition to the protein abundance of the anterograde promoting transport, unknown and/or poorly characterized proteins identified (Protein ID: G5EBP5, Q9N4I3 and 
Q8MXD9) were found to be up-regulated, in which Q8MXD9 (E02D9.1, isoform b) uncharacterized was represented as a physical interaction partner of GPB-1, shown to be up-regulated in this study, in humans and yeast by the program of functional protein association networks (STRING 9.1). The other Q9N4I3 has been predicted to regulatory function ubiquitin-associated domains (UBA)-like in Gene Ontology. With this, RAD-23 known as a protein of Radiation Sensitivity 23 family of proteasomal ubiquitin receptors was quantitatively up-regulated in unc-50 mutants. It also interacts physically with PNGases (Peptide:N-glycanases, cytoplasmic de-N-glycosylation enzymes) facilitating the degradation of misfolded protein substrates during endoplasmic reticulum-associated degradation (ERAD) in yeast and mammals and regulates neuronal axon branching in the vulva of $C$. elegans (Habibi-Babadi et al., 2010). Besides, UCR-2.3 (UbiquinolCytochrome c oxido-reductase known as mitochondrial electron transport complex III), PBRM-1 (Polybromo 1 involved in transcriptional regulation of select genes by chromatin remodeling and metal ion binding in Gene Ontology) and H2B1 (Histone $\mathrm{H} 2 \mathrm{~B} 1$ as chromatin components) were appeared to be up-regulated in the SILAC ratios of unc-50 mutants (Table VII.2A and B).

In contrast, the proteins shown to be down-regulated in the SILAC ratios were represented in Table VII.2C and D. VIT-3 (Vitellogenin-3, yolk and lipid binding proteins), C27F2.10 (Uncharacterized, proteasome component (PCI) domain-containing protein 2) (Wei et al., 2008) and BICD-1 (Bicaudal D homolog dynein binding proteins) required for the retrograde migration of Rab6-positive vesicles to the perinuclear region and the Golgi-to-ER as a dynein-mediated transport along microtubules were down regulated in both replicates (T1 and $\mathrm{T} 2$ ). As a fold change of SILAC ratio at least in one replicate shown to be down-regulated, VIT-5 (Vitellogenin-5, yolk and lipid transport proteins), Q22531 (Unknown, putative metallopeptidase activity and zinc ion binding in Gene Ontology), CTH2 (Putative cystathionine gamma-lyase 2, function of iron savings and adaptive advantages to iron deficiency in loss of function) (Cyert and Philpott, 2013), P34385 (Uncharacterized, probable methylcrotonoyl-CoA carboxylase beta chain), Q6BEV6 (Unknown, F49E2.5), DOD-19 (Lifespan extension related proteins) (Murphy et al., 2003) and O61880 (Uncharacterized, lifespan extension related proteins) (Li et al., 2004) were included (Table VII2.C). 
These results indicate that the lack of UNC-50 in C. elegans causes quantitative protein changes of transport-related factors, in which the active forward movement of vesicles and/or organelles seems to be dependent on the motor-driven actin-myosin pathways. Whereas the dynein-mediated retrograde-oriented movement factors are suppressed in the absence of UNC-50, supporting that UNC-50 does act as a trafficking regulator between intracellular compartments.

Moreover, quantitative protein abundance of GPB-1, UNC-18 and VPS-32.1, which is involved in the anterograde promoting transport as endosome-mediated trafficking manners in C. elegans and yeast (McEwen and Kaplan, 2008; Thyagarajan et al., 2011), is likely to disturb the balance of traffic-dynamic flow in the subcellular compartments due to the lack of the retrograde regulators.

Taken together, the absence of UNC-50 leads to quantitative protein abundance of anterograde pronounced transport and, at the same time, protein poverty of retrograde transport regulators, occurring that protein recycling, secretion and membrane-mediated trafficking are affected in $u n c-50$ mutants.

A

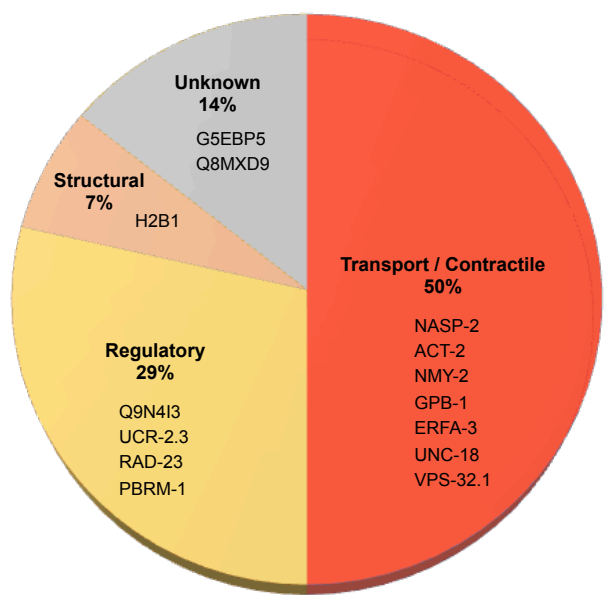

Proteins up-regulated in unc-50 mutants

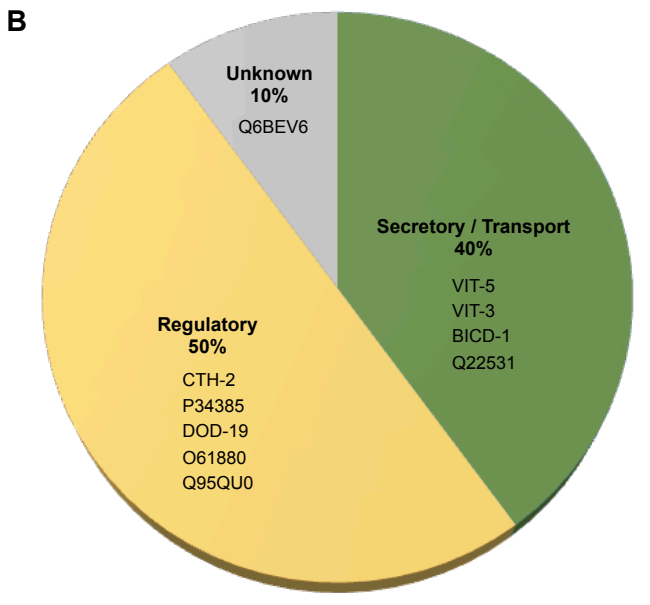

Proteins down-regulated in unc-50 mutants

Figure VII.6 Functional classification of the proteins identified in unc-50 mutants. Pie charts showing the groups of proteins un-regulated (A) and down-regulated (B) in unc-50 mutants. Based on the experimental evidence and functional protein information of UniProt (the Universal Protein Resource, www.uniprot.org), WormBase, yeast genome database (SGD) and/or Ensemble, functional categories of proteins were classified into seven groups: Transport, Regulatory, Structural, Contractile, Catalytic, Immunological and Secretory. 


\begin{tabular}{|c|c|c|c|c|c|c|c|}
\hline Protein ID & Name & Description & $\begin{array}{l}\text { Functional } \\
\text { Catagories }\end{array}$ & Source & Peptides & $\begin{array}{l}\text { Norm. Ratio } \\
\text { WTH/MUL }\end{array}$ & $\begin{array}{l}\text { Norm. Ratio } \\
\text { WTH/MUL }\end{array}$ \\
\hline
\end{tabular}

A Proteins with a fold change of SILAC ratios $<0.5$ in both replicates T1 and T2 (up-regulated)

replicate $1 \quad$ replicate 2

\begin{tabular}{|c|c|c|c|c|c|c|c|}
\hline O17687 & NASP-2 & $\begin{array}{l}\text { Nuclear autoantigenic sperm protein (histone-binding } \\
\text { protein); highly conserved proteins in eukaryotes; } \\
\text { transport of histons ; SHNi-TPR (Sim3-Hif1-NASP } \\
\text { interrupted TPR) protein family (Ref. 1) }\end{array}$ & Transport & Ensembl & 14 & 0,39 & 0,47 \\
\hline
\end{tabular}

B Proteins with a fold change of SILAC ratios $<0.5$ in one replicate and a fold change of SILAC ratios $<1$ in the other (up-regulated)

\begin{tabular}{|c|c|c|c|c|c|c|c|}
\hline P10984 & ACT-2 & $\begin{array}{l}\text { Actin-2; cell motility; ubiquitously expressed in all } \\
\text { eukaryotic cells; contractile filaments in muscles }\end{array}$ & $\begin{array}{c}\text { Contractile / } \\
\text { Transport }\end{array}$ & $\begin{array}{l}\text { UniProt, } \\
\text { WormBase }\end{array}$ & 24 & 0,85 & 0,49 \\
\hline G5EBY3 & NMY-2 & $\begin{array}{l}\text { Non-muscle myosin; motor proteins; coiled-coil and } \\
\text { myosin-like IQ motif-containing domains }\end{array}$ & $\begin{array}{c}\text { Contractile / } \\
\text { Transport }\end{array}$ & $\begin{array}{l}\text { UniProt, } \\
\text { WormBase }\end{array}$ & 19 & 0,49 & 0,79 \\
\hline Q9N4I3 & Y71F9AL.9 & $\begin{array}{l}\text { Uncharacterized; UBA (ubiquitin-associated domains)- } \\
\text { like (Molecular function, IEA*) }\end{array}$ & Regulatory & Ensembl & 8 & 0,44 & 0,77 \\
\hline P17343 & GPB-1 & $\begin{array}{l}\text { Guanine nucleotide-binding protein subunit beta-1; } \\
\text { endosomal membranes; trafficking to the anterior-side } \\
\text { in early cell division axes (Ref. 2) }\end{array}$ & $\begin{array}{c}\text { Regurator/ } \\
\text { Transport }\end{array}$ & $\begin{array}{l}\text { UniProt, } \\
\text { WormBase }\end{array}$ & 4 & 0,77 & 0,42 \\
\hline O45622 & ERFA-3 & $\begin{array}{l}\text { Eukaryotic Release FActor homolog; phi-19; GO } \\
\text { (Molecular function, IEA*), GTPase activity, GTP } \\
\text { binding }\end{array}$ & $\begin{array}{c}\text { Regurator/ } \\
\text { Transport }\end{array}$ & $\begin{array}{l}\text { UniProt, } \\
\text { WormBase }\end{array}$ & 7 & 0,88 & 0,49 \\
\hline Q9TZ33 & UCR-2.3 & $\begin{array}{l}\text { Ubiquinol-Cytochrome c oxidoReductase complex; bc1 } \\
\text { complex; mitochondrial electron transport complex III }\end{array}$ & Regulatory & WormBase & 10 & 0,49 & 0,58 \\
\hline Q8MXD9 & E02D9.1 & Unknown; E02D9.1, isoform b & - & - & 8 & 0,47 & 0,69 \\
\hline G5EEY5 & PBRM-1 & $\begin{array}{l}\text { Polybromo } 1 \text {; transcriptional regulation of select genes } \\
\text { by chromatin remodeling }\end{array}$ & Regulatory & $\begin{array}{l}\text { UniProt, } \\
\text { WormBase }\end{array}$ & 2 & 0,38 & 0,67 \\
\hline Q18886 & VPS-32.1 & Related to yeast vacuolar protein sorting factor & Transport & WormBase & 3 & 0,67 & 0,36 \\
\hline P04255 & $\mathrm{H} 2 \mathrm{~B} 1$ & $\begin{array}{l}\text { Histone H2B family; Core histones; his-11; his- } 4 \text {; his- } \\
48 \text {; his- } 41 \text {; his- } 39 \text {; components of chromatin }\end{array}$ & Structural & UniProt & 2 & 0,60 & 0,43 \\
\hline
\end{tabular}

C Proteins with a fold change of SILAC ratios $>2$ in one replicate and a fold change of SILAC ratios $>1$ in the other (down-regulated)

\begin{tabular}{|c|c|c|c|c|c|c|c|}
\hline P06125 & VIT-5 & $\begin{array}{l}\text { VITellogenin-5; VITellogenin structural genes (yolk } \\
\text { protein genes); lipid transport proteins; Secreted into } \\
\text { the body cavity from intestine hermaphrodite and } \\
\text { subsequently taken up by the gonad (oocytes) (Ref. } 4 \\
\text { and 5) }\end{array}$ & $\begin{array}{l}\text { Structural/ } \\
\text { Secretory }\end{array}$ & $\begin{array}{l}\text { UniProt, } \\
\text { WormBase }\end{array}$ & 94 & 1,90 & 2,06 \\
\hline Q22531 & T16G12.1 & $\begin{array}{l}\text { Unknown; GO_(Molecular function, IEA*), } \\
\text { metallopeptidase activity, zinc ion binding }\end{array}$ & $\begin{array}{l}\text { Catalytic / } \\
\text { Scretory? }\end{array}$ & UniProt & 14 & 2,07 & 1,77 \\
\hline P55216 & $\mathrm{CTH}-2$ & $\begin{array}{l}\text { Putative cystathionine gamma-lyase } 2 \text {; mRNA-binding } \\
\text { protein expressed during iron starvation }\end{array}$ & Regulatory & UniProt & 6 & 2,86 & 1,69 \\
\hline P34385 & F02A9.4 & $\begin{array}{l}\text { Uncharacterized; Probable methylcrotonoyl-CoA } \\
\text { carboxylase beta chain; mitochondrial }\end{array}$ & Regulatory & UniProt & 8 & 4,41 & 1,08 \\
\hline Q6BEV6 & F49E2.5 & Unknown; F49E2.5, isoform $\mathrm{h}$ & - & - & 7 & 2,49 & 1,95 \\
\hline O61874 & DOD-19 & Membrane raft; related to lifespan extension (Ref. 6) & Regulatory & UniProt & 4 & 4,83 & 1,00 \\
\hline O61880 & F59B1.2 & $\begin{array}{l}\text { uncharacterized; ddl-1 binding proteins related to } \\
\text { lifespan (Ref. 7) }\end{array}$ & Regulatory & WormBase & 1 & 2,09 & 1,96 \\
\hline Q95QU0 & $\mathrm{C} 27 \mathrm{~F} 2.10$ & $\begin{array}{l}\text { Proteasome component }(\mathrm{PCI}) \text { domain-containing } \\
\text { protein } 2 \text { homolog; COP9 signalosome (CSN) 12-like } \\
\text { protein; ubiquitin-proteasome pathway (Ref. } 8 \text { ) }\end{array}$ & Regulatory & UniProt & 3 & 2,66 & 4,11 \\
\hline V6CK55 & BICD-1 & $\begin{array}{l}\text { Bicaudal D (Drosophila) homolog; dynein-mediated } \\
\text { tranport; dynein regulators; nuclear migrations; } \\
\text { microtubule anchorage at the centrosome; Rab6- } \\
\text { secretory vesicles to the pericentrosome and Golgi-ER } \\
\text { retrograde transport (Ref. } 9,10 \text { and 11) }\end{array}$ & $\begin{array}{l}\text { Transport / } \\
\text { Secretory }\end{array}$ & WormBase & 3 & 2,79 & 2,88 \\
\hline
\end{tabular}


Table VII.2 Quantitative protein abundance and down-regulation of intracellular transport related factors in $\mathbf{u n c - 5 0}$ mutants. Twenty-four proteins were identified with a fold change in SILAC ratios in the absence of UNC-50. The quantitative protein abundances normalized in the control are shown in replicator 1 and replicator 2. Proteins identified were sorted in order (A - D) as up-regulated proteins (total 14) of a fold change (SILAC ratios $<0.5$ ) and down-regulated proteins (total 10) of a fold change (SILAC ratio > 2) from at least one experimental replicator. IEA* stands for Inferred from electronic annotation in Gene Ontology (GO). Ref. 1: (NabeelShah et al., 2014), Ref. 2: (Thyagarajan et al., 2011), Ref. 3: (McEwen and Kaplan, 2008), Ref. 4: (Spieth and Blumenthal, 1985), Ref. 5: (Kamath et al., 2003), Ref. 6: (Murphy et al., 2003), Ref. 7: (Li et al., 2004), Ref. 8: (Wei et al., 2008). Ref. 9, 10 and 11: (Matanis et al., 2002; Schlager et al., 2010; Terenzio and Schiavo, 2010). Source: The SILAC ratios normalized were provided from a collaborator, Sebastian Wiese, Laboratory of Proteomics, Uni-Ulm.

\subsection{Metabolic analysis of the mutants of unc-50 in C. elegans}

C. elegans metabolic processes are highly similar to humans in the process of biosynthesis and breakdown of dietary sources and energy storage as lipids (Table VI.1) (Braeckman et al., 2009; Szewczyk et al., 2003).

Based on the phenotypes by HPF-EM, unusual large lipid-droplets like vesicular structures are observed in the muscle cytoplasm in unc-50 mutants that may indicate defects of metabolic processes. In order to see differential metabolic intermediates between wild type and unc-50 mutants, the unbiased whole metabolome profiling was initiated in collaboration with the ZBSA Core Facility Metabolomics, Uni-Freiburg. As shown in Table VII.3, an initial metabolic profiling of unc-50 mutants revealed that the levels of a total of 47 metabolites were significantly altered compared to wild type, in which the ratios of fold-change in order were present from large to small values. In the results, major metabolic changes in KEGG pathways indicated in carbohydrate/nucleotide/amino acid metabolisms that may be affected in unc-50 mutants in C. elegans. In particular, we detected strong increase in the levels of phosphoenolpyruvate (PEP), urate, L-asparagine and alpha-trehalose whereas (S)allantoin, sucrose, GMP, fumarate, anthranilate and xanthine were found to be decreased. This suggests that the catabolic pathway from urate to allantoin through urate oxidase 
reaction may be blocked so that it causes increased urate and decreased allantoin in unc50 mutants. Therefore UNC-50 may affect in the urate metabolism.

\begin{tabular}{|c|c|c|c|c|}
\hline KEGG_NAME & KEGG_ENTRY & Fold-change & SD & $p$ value ( $t$-test) \\
\hline Phosphoenolpyruvate & $\mathrm{C} 00074$ & 2,94 & 0,19 & 0,03 \\
\hline Urate & C00366 & 2,47 & 0,10 & 0,00 \\
\hline L-Asparagine & C00152 & 1,84 & 0,11 & 0,01 \\
\hline alpha,alpha-Trehalose & C01083 & 1,74 & 0,06 & 0,00 \\
\hline L-Glutamine & C00064 & 1,45 & 0,15 & 0,05 \\
\hline L-Serine & C00065 & 1,28 & 0,06 & 0,01 \\
\hline Ethanolamine phosphate & C00346 & 1,21 & 0,10 & 0,04 \\
\hline sn-Glycerol 3-phosphate & $\mathrm{C} 00093$ & 0,92 & 0,05 & 0,03 \\
\hline Pantothenate & C00864 & 0,91 & 0,04 & 0,01 \\
\hline Guanosine & C00387 & 0,90 & 0,03 & 0,01 \\
\hline Citrate & C00158 & 0,90 & 0,05 & 0,04 \\
\hline Sedoheptulose 7-phosphate & C05382 & 0,88 & 0,05 & 0,00 \\
\hline Benzoate & C00180 & 0,87 & 0,06 & 0,05 \\
\hline D-Mannose 6-phosphate & C00275 & 0,86 & 0,03 & 0,00 \\
\hline beta-D-Glucose 6-phosphate & C01172 & 0,84 & 0,05 & 0,00 \\
\hline L-Isoleucine & C00407 & 0,84 & 0,03 & 0,05 \\
\hline (9Z)-Octadecenoic acid & $\mathrm{C} 00712$ & 0,83 & 0,04 & 0,00 \\
\hline L-Ornithine & $\mathrm{C} 00077$ & 0,81 & 0,05 & 0,01 \\
\hline L-Aspartate & C00049 & 0,80 & 0,03 & 0,00 \\
\hline Icosanoic acid & C06425 & 0,79 & 0,10 & 0,02 \\
\hline Octadecanoic acid & C01530 & 0,78 & 0,07 & 0,02 \\
\hline Nicotinate & $\mathrm{C} 00253$ & 0,78 & 0,10 & 0,04 \\
\hline Cadaverine & C01672 & 0,76 & 0,06 & 0,00 \\
\hline L-Methionine & $\mathrm{C} 00073$ & 0,75 & 0,11 & 0,02 \\
\hline (S)-Lactate & C00186 & 0,74 & 0,07 & 0,00 \\
\hline D-Ribose 5-phosphate & C00117 & 0,74 & 0,06 & 0,00 \\
\hline Diphosphate & $\mathrm{C} 00013$ & 0,74 & 0,04 & 0,01 \\
\hline beta-D-Fructose & $\mathrm{C} 02336$ & 0,74 & 0,03 & 0,00 \\
\hline AMP & $\mathrm{C} 00020$ & 0,74 & 0,03 & 0,00 \\
\hline L-Tyrosine & C00082 & 0,73 & 0,06 & 0,03 \\
\hline Hexadecanoic acid & C00249 & 0,72 & 0,06 & 0,00 \\
\hline 6-Phospho-D-gluconate & $\mathrm{C} 00345$ & 0,71 & 0,05 & 0,00 \\
\hline Putrescine & C00134 & 0,70 & 0,06 & 0,00 \\
\hline Spermidine & C00315 & 0,70 & 0,14 & 0,02 \\
\hline Linoleate & C01595 & 0,70 & 0,09 & 0,00 \\
\hline 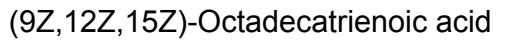 & C06427 & 0,69 & 0,07 & 0,00 \\
\hline D-Ribose & $\mathrm{C} 00121$ & 0,68 & 0,06 & 0,00 \\
\hline Pyruvate & $\mathrm{C} 00022$ & 0,64 & 0,07 & 0,00 \\
\hline Adenine & C00147 & 0,62 & 0,04 & 0,00 \\
\hline Hydroxylamine & C00192 & 0,62 & 0,12 & 0,03 \\
\hline Oxalate & C00209 & 0,62 & 0,10 & 0,00 \\
\hline Xanthine & C00385 & 0,60 & 0,05 & 0,00 \\
\hline Anthranilate & C00108 & 0,53 & 0,08 & 0,00 \\
\hline Fumarate & C00122 & 0,47 & 0,02 & 0,00 \\
\hline GMP & C00144 & 0,43 & 0,18 & 0,00 \\
\hline Sucrose & C00089 & 0,28 & 0,23 & 0,04 \\
\hline$(\mathrm{S})(+)$-Allantoin & $\mathrm{C} 02350$ & 0,02 & 0,00 & 0,00 \\
\hline
\end{tabular}

Table VII.3 Metabolic profiles of unc-50 mutants. C. elegans unc-50 mutants were compared to wild type in a fold change, in which forty-seven compounds were observed to be significantly 
changed. Source: Results sorted by the author were provided from Carsen Jäger in ZBSA, Core Facility of Metabolomics, Uni-Freiburg.

\subsection{Functional expression of Bir A* fusion proteins in $C$. elegans muscles}

Protein-protein interactions in intracellular trafficking pathways are very dynamic as both core machinery components and cargo proteins interact only transiently during their transport . As described in Chapter I, UNC-50 may act as a trafficking regulator involved in the ARF-dependent retrograde membrane trafficking at the Golgi-endosomal interface. To identify UNC-50 interacting proteins in C. elegans, in vivo UNC-50 has to be tagged and purified with the interacting proteins. To reduce the number of non-specific contaminants, a high-affinity purification system has to be applied that is compatible with SILAC labeling for MS analysis.

For this purpose, we chose to use BioID, a proximity-dependent biotinylation method to identify intracting protein. We adopted this technique to isolate UNC-50 interaction partner and expressed the UNC-50::BirA* fusion protein specifically in the muscle cell of unc-50 (x47) mutant animals (Fig. VII.7).

To evaluate the functional expression of BirA* fusion proteins that were integrated into unc-50 (x47) III mutants, we investigated the worms by $1 \mathrm{mM}$ levamisole plates, microscopy and biochemical assays to confirm whether they are functional endogenously in C. elegans. The strains of UNC-50::BirA* were shown to be paralyzed like wild type after two-hour on $1 \mathrm{mM}$ levamisole plates, meaning that the $u n c-50$ mutants were rescued by the UNC-50::BirA* to be hyper-contracted on $1 \mathrm{mM}$ levamisole plates (Fig. VII.8A). This suggests that the UNC-50::BirA* fusion construct is functional in C. elegans.

As control constructs, we tested BirA* to be soluble when fused to GFP and anti-GFP nanobody ( $\alpha$ GFPNB), respectively, whether they are functionally expressed without any proteolytic processing. As shown in Figure VII.8B and B', GFP.:BirA* were apparently expressed in the body wall muscles as shown by microscopy. At the C-terminus of $\alpha$ GFPNB containing c-myc-tags $(1.2 \mathrm{kDa})$, the BirA* was fused to be endogenously expressed in unc-50 (x47) mutants. The aGFPNB::BirA* containing c-myc-tags was detected to be approximately $\sim 50 \mathrm{kDa}$ with $\alpha$-c-myc by Western blots (Fig. VII.8C), 
indicating that all the BirA* fusion proteins function properly in C. elegans. Any defects were not found in the strains of the BirA* fusion proteins integrated into unc-50 (x47) mutants.

\section{BirA* fusion constructs}

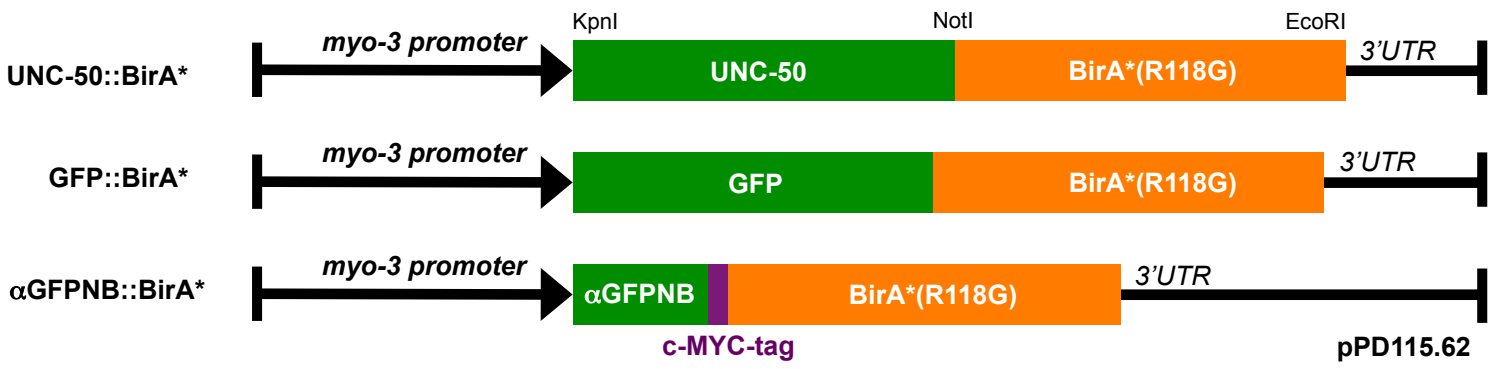

Figure VII.7 Schematic representation of BirA*-fusion proteins to be integrated into unc-50 mutants. As proteins of interest UNC-50, GFP and anti-GFP nanobody ( $\alpha$ GFPNB) that contains c-myc tags were subcloned into the expression vector pPD115.62. Transgenic worm lines induced by extrachromosomal arrays were subsequently integrated by gamma irradiation to be expressed endogenously in the worm strains gzIs20, gzIs 21 and gzIs62. Each clone of the plasmid constructs was confirmed by sequencing.

A

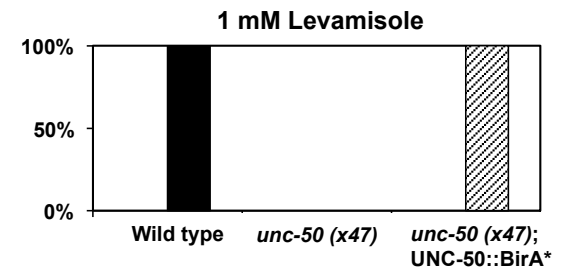

C

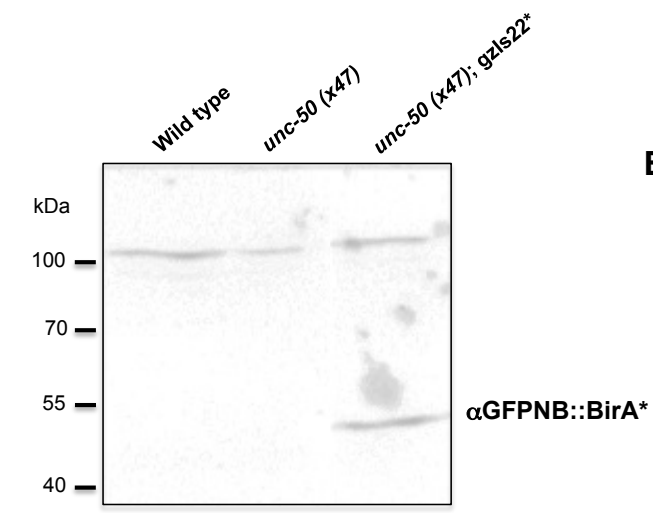

B

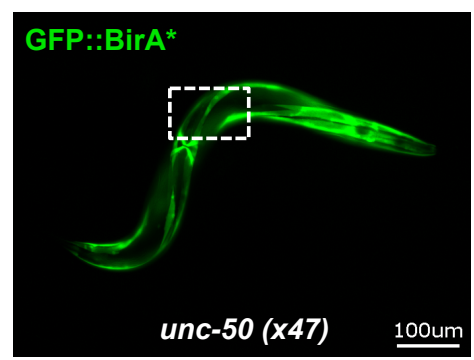

B'

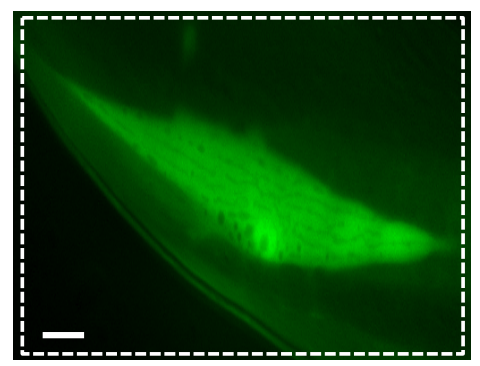

Figure VII.8 Functional expression of BirA*-fusion proteins in $C$. elegans muscles. Integrated worm strains unc-50 (x47) III; gzIs20 [pmyo-3::unc-50::BirA*], unc-50 (x47) III;

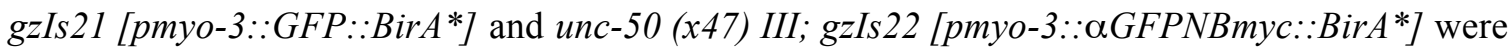


used in this study that were three-time outcrossed with N2 wild types. The gzIs60 stains were tested on the $1 \mathrm{mM}$ levalmisole plates for two hours. Thirty worms in total were $100 \%$ rescued by three independent experiments (A). The functional expression of GFP::BirA* into unc-50 mutants was evaluated by microscopy (BZ-9000 BIOREVO by Keyence). The dashed boxes in order (B and B') indicate magnified images of the gzIs 21 expressed in the body wall muscles. Scale bars, $10 \mu \mathrm{m}$. By using the $\alpha-\mathrm{c}-\mathrm{MYC}(200 \mathrm{ng} / \mathrm{ml})$ antibodies, the protein size of the BirA* fused to the anti-GFP nanobodies containing c-myc tags ( $\alpha$ GFPNB::BirA*, approx. $\sim 50 \mathrm{kDa}$ ) was confirmed by Western blots. (BirA*, $35.5 \mathrm{kDa}$; $\alpha$ GFPNB, 12-15 kDa and c-MYC tags, $1.2 \mathrm{kDa}$ ) (C).

\subsection{Workflow strategy of SILAC-BioID in C. elegans}

UNC-50/GMH1 is a physical binding partner of ARF-GEFs GEA1/2 containing a conserved SEC7 domain in yeast, nematode and human (Chantalat et al., 2003; Eimer et al., 2007), indicating UNC-50/GMH1 is likely to be involved in the intracellular trafficking as ARF-GEF-dependent manners that may undergo highly dynamic and transient protein interactions in the intracellular compartments.

To identify the interaction proteins of UNC-50, it was initially labeled by BirA* (Fig. VII.7). Since all the BirA*-fusion proteins were functional in vivo as shown in the section of VII.5, we designed the proteomic experiments MS-based in C. elegans for finding specific interaction proteins of UNC-50 by using metabolic heavy/light-isotope labeled essential amino acids (SILAC) combined with BioID. As described previously, the BioID is based on promiscuous biotinylated-protein identification to be subsequently purified in affinity isolation of specific proteins with streptavidin-coated matrixes prior to be identified by MS analysis (Fig. VII.9). In the process, the lines expressing BirA* fusion proteins that have been confirmed to be endogenously functional in vivo (Fig. VII.8) gzIs20 (UNC-50::BirA*), gzIs21 (GFP::BirA*) and gzIs22 (〈GFPNB::BirA*) were SILAC labeling via feeding SLE1 bacteria $\left(50\right.$-fold, $\mathrm{OD}_{600 \mathrm{~nm}}$ of 1) containing $50 \mu \mathrm{M}$ biotin (final conc.) in each light-(Lys0, Arg0) and heavy-(Lys8, Arg10) SILAC plate (Fig. VIII.9).

To validate whether endogenous biotinylated proteins are detected in C. elegans, whole worm lysates were analyzed by streptavidin-conjugated horseradish peroxidase (HRP) blots (10 minutes exposure, $250 \mathrm{ng} / \mathrm{ml}$, Thermo scientific) (Fig. VII.10). As shown in the 
controls (lanes 1 and 2), endogenously biotinylated proteins that do not have any BirA*fusion proteins in worm strains were revealed to be approximately $80 \mathrm{kDa}$ in size in $C$. elegans, which is similar to the endogenously biotinylated proteins in humans $(80.8 \mathrm{kDa})$ and yeast $(76.4 \mathrm{kDa})$ as a monomeric size (Chapman-Smith and Cronan, 1999; Rhee et al., 2013). The worm lysates of SILAC labeling with biotin supplements during the growth were shown to be strongly biotinylated by streptavidin-HRP blotting, suggesting that the SILAC strains containing BirA*-fusion proteins with $50 \mu \mathrm{M}$ biotin were endogenously biotinylated in the interacting proteins of the proteins of interest (UNC-50, GFP and $\alpha$ GFPNB) as a proximity-dependent manner. Taken together, the results suggest that UNC-50::BirA fusion protein biotinylates in vivo and can then be used for BioID experiments. 


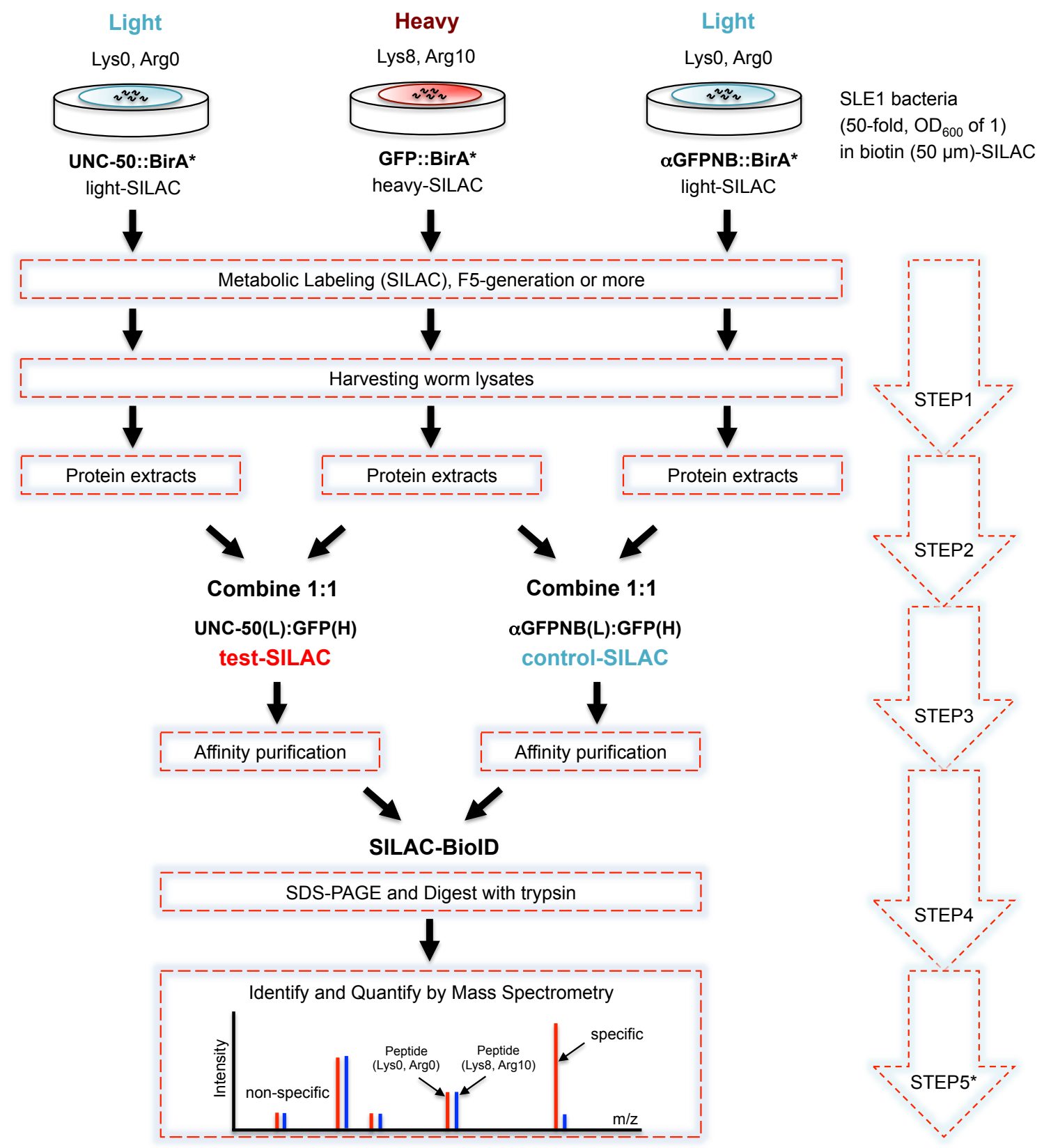

Figure VII.9 Schematic workflow of SILAC-BioID in C. elegans. Experimental processes of SILAC-combined affinity-pulldown experiments are to identify novel UNC-50-binding proteins by high-affinity biotinylated proteins captures using streptavidin-coated magnetic beads in pulling conditions, which can be divided into five steps. In STEP1, the worm strains gzIs20 (UNC-

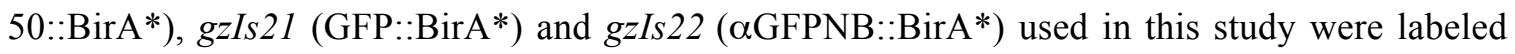
by SILAC via feeding SLE1 bacteria to be fully incorporated isotopes into the proteomes. Based on the metabolic incorporation experiments in the section of VII.2, the worms are subjected to maintain in the SILAC plates at least until F5-generation or more. Harvesting worm populations 
each by each before frozen worms were done with ice-cold-PBS containing protease inhibitors (Appendix) and extracted proteins according to the described previously were combined to be equal amounts of each in STEP2 (Roux et al., 2012). The test-SILAC [UNC-50(L):GFP(H)] and control-SILAC $[\alpha \operatorname{GFPNB}(\mathbf{L}) \operatorname{GFP}(\mathbf{H})]$ in STEP3 were immunoprecipitated by affinity-pulldown isolation steps using streptavidin-coated magnetic beads (Dynabeads MyOne C1, Invitrogen). Triplicated test-SILAC samples and control-SILAC were subjected to 10\% SDS-PAGE in STEP4 and then samples were treated to be MS analysis that was conducted by collaboration in this study. STEP1-4 were done by the author and STEP5* is ongoing in collaboration with Sebastian Wiese, Laboratory of proteomics, Uni-Ulm.

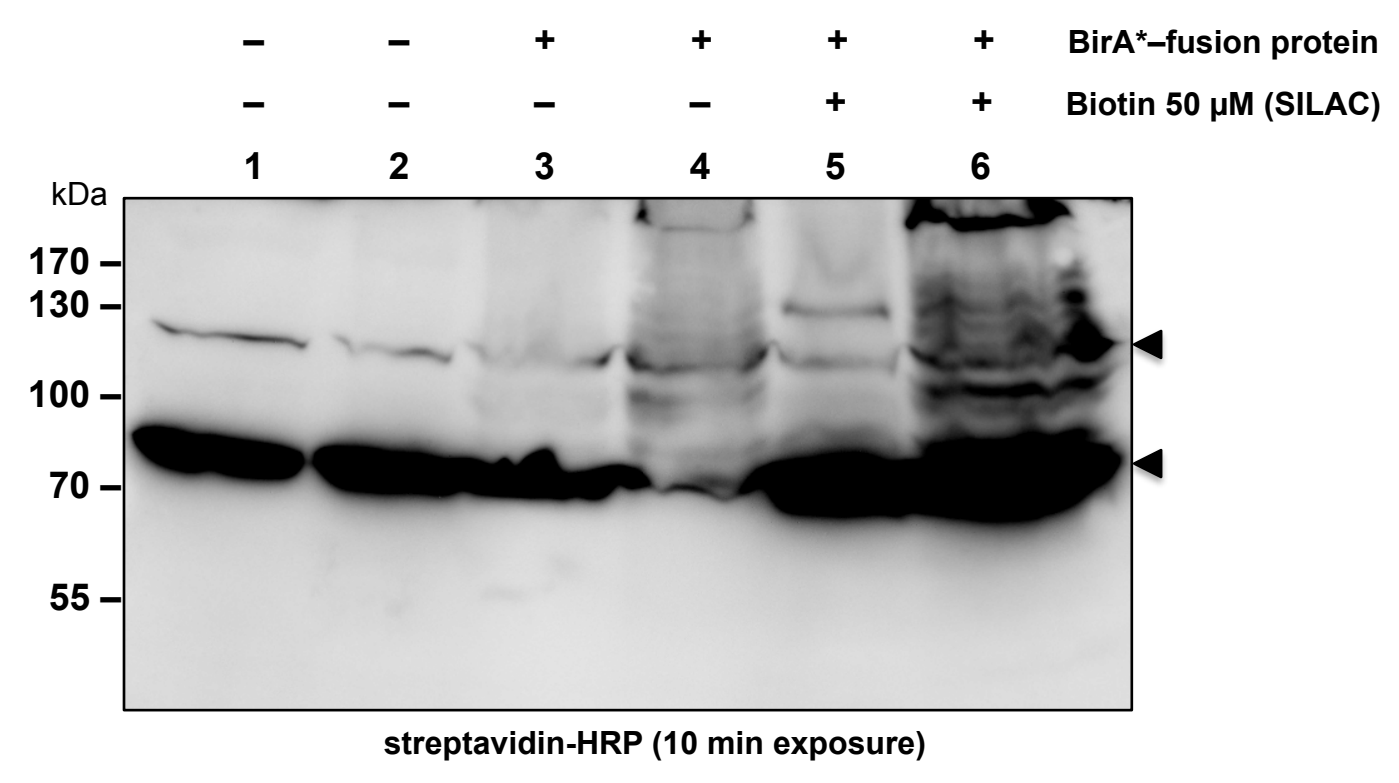

Figure VII.10 Analysis of the endogenously biotinylated proteins in C. elegans. Whole cell worm lysates were analyzed to confirm endogenous biotinylated proteins in controls (lane 1, wild type; lane 2, unc-50 (x47) mutant), endogenous BirA*-fusion protein-containing worms (lane 3, gzIs21 (unc-50 (x47) III; gzIs21 [pmyo-3::GFP::BirA*]); lane 4, gzIs22 (unc-50 (x47) III; gzIs22

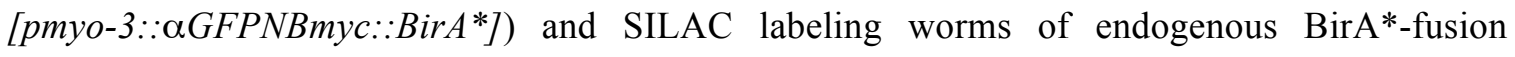
proteins in $50 \mu \mathrm{M}$ biotin plates (lane 5, gzIs20 (unc-50 (x47) III; gzIs20 [pmyo-3::unc-

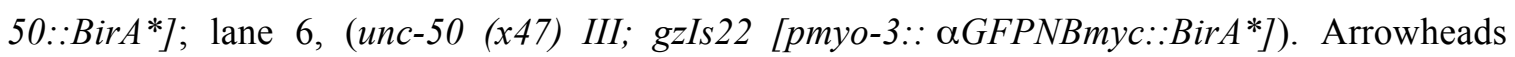
indicate endogenously biotinylated proteins (approx. $80 \mathrm{kDa}$ and $120 \mathrm{kDa}$ ) present in all worm lysates. The worm lysates (lane 3) containing BirA*-fusion proteins without treatment of $50 \mu \mathrm{m}$ biotin are shown to be similar blots compared to the controls (lanes 1 and 2) and detected in the extensively modified molecular sizes that seems to be endogenously biotinylated in lane 4 . In the 
SILAC labeling samples (lanes 5 and 6) with $50 \mu \mathrm{m}$ biotin, lots of biotinylated proteins are shown in the streptavidin-HRP blots, 10 minutes (min) exposures.

\subsection{Functional specificity of the GFP nanobodies in the intracellular compartment via spatially labeling of the lumen and cytosol in $C$. elegans muscles}

Based on the matters that are associated with the generation of fusion protein, we wanted to determine whether an anti-GFP nanobody ( $\alpha$ GFPNB)::BirA fusion protein could be used as a universal in vivo affinity reagent to be used with GFP-tagged target protein for BioID applications. First, we tested the functionality of the intracellular detection of GFP fusion protein by the $\alpha$ GFPNB in vivo. For this purpose, we coexpressed GFP-fusion protein and $\alpha$ GFPNB::tagRFPt fusion construct in order to confirm whether the GFP fusion protein could be detected with $\alpha$ GFPNB::tagRFPt fusion construct in C. elegans (VII.11). The GFP-fusion proteins were targeted to different subcellular compartment such as the ER, Golgi (Fig. VII.12), Mitochondria (outer and matrix) (Fig. VII.13), Nucleus and Synapse at NMJs (Fig. VII.14). Furthermore, we also tested GFP-fusion proteins where the GFP-tag was placed within the lumen at the ER or Golgi.

The anti-GFP Nanobodies $(12-15 \mathrm{kDa})$ are a small antibody fragment $(2.5 \mathrm{~nm}$ in diameter and $4.2 \mathrm{~nm}$ in length) as a single variable domain known as heavy chain-only antibodies of Camelidae family (Chakravarty et al., 2014; Kubala et al., 2010; Rothbauer et al., 2008; Rothbauer et al., 2006). The antigen GFP binding specificity of the singledomain nanobodies ( $\alpha$ GFPNB) was reported as chromobodies and/or GFP-nanotrap complexes that can be stably expressed in living cells (Rothbauer et al., 2008; Rothbauer et al., 2006). With this, studying the $\alpha$ GFPNB complexes based on the structural binding analysis revealed that the conformation/manipulation of the complexes can be monitored in protein expression of subcellular localization as well as translocation of proteins in living cells, that led us to study the functional-spatial restriction of the $\alpha$ GFPNB in the complex with the antigen GFP in the intracellular compartments in the living organism, C. elegans (Kirchhofer et al., 2010; Rothbauer et al., 2008; Rothbauer et al., 2006; Schornack et al., 2009). 
For this purpose, the constructs of the $\alpha$ GFPNB were subcloned into the expression vector pPD115.62 (Appendix). The aGFPNB was fused to the tagRFPt, a photostable red-fluorescent protein (Fig. VII.10), to be tested with the GFP/YFP/CFP fusion proteins labeled at the $\mathrm{N}$ - or C-terminus of the organelle specific proteins. We tested whether the complex conformation of GFP- $\alpha$ GFPNB are affected in functional-spatial restriction by the membrane-barrier of the subcellular compartments (Fig. VII.12, 13 and 14).

A

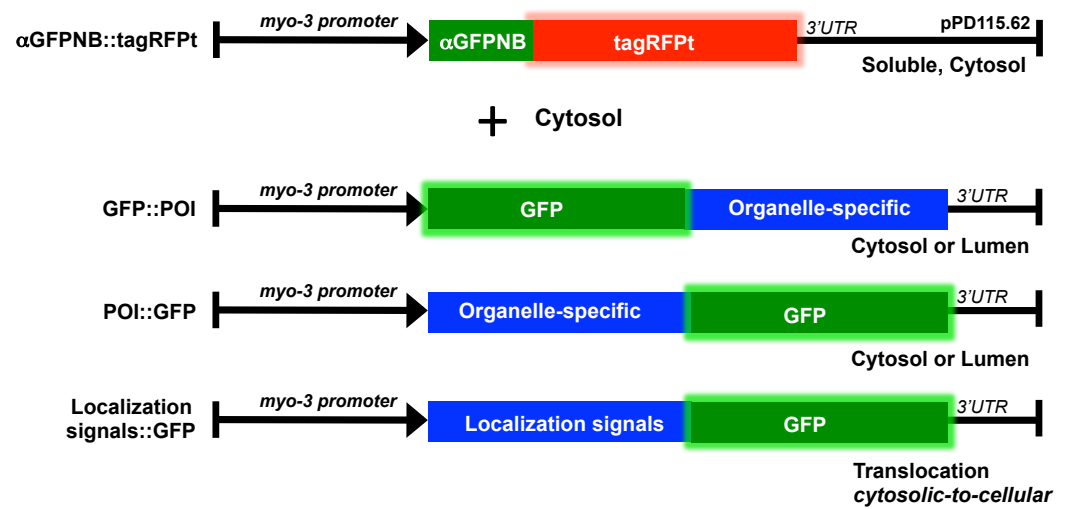

B

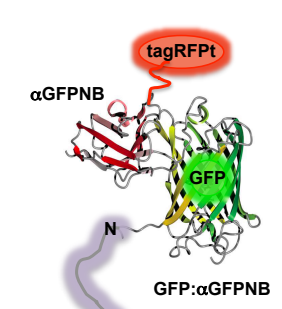

Figure VII.11 Schematic representation of soluble $\alpha$ GFPNB::tagRFPt and GFP-fusion organelle specific proteins. In order to be co-expressed in C. elegans muscles, we constructed $\alpha$ GFPNB::tagRFPt and GFP-fusion organelle specific proteins to test whether the complexes are functional to be colocalized through the organelle membrane barriers (A). In this study, we used the fluorescent-fusion organelle markers in either cytosolic- and/or lumen/cellular-faced domain structures. For example, an organelle marker as a type II of transmembrane shows GFP-labeled at the end of N-terminus targeted to the cytosol while the C-terminus of the protein faces to the ER lumen (B). Source: crystallization structures of the GFP- $\alpha$ GFPNB complexes were taken from the reference (Kubala et al., 2010). 
A
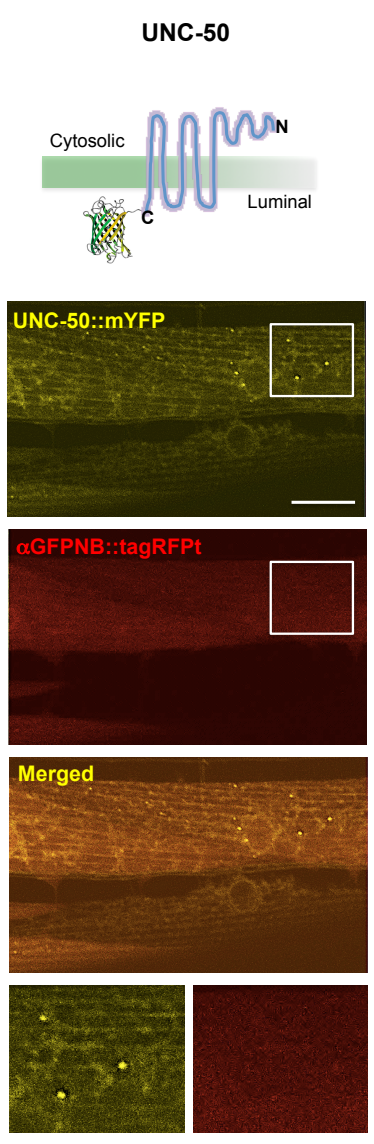

Not Colocalized

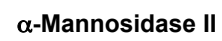
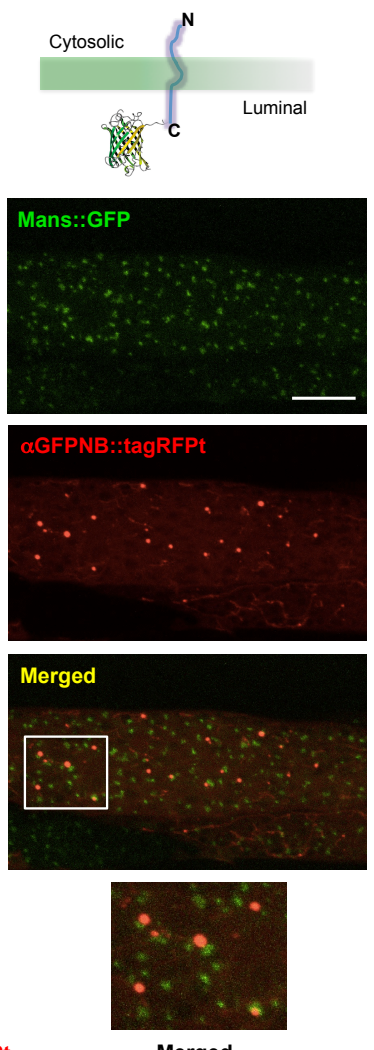

Merged Not Colocalized
C
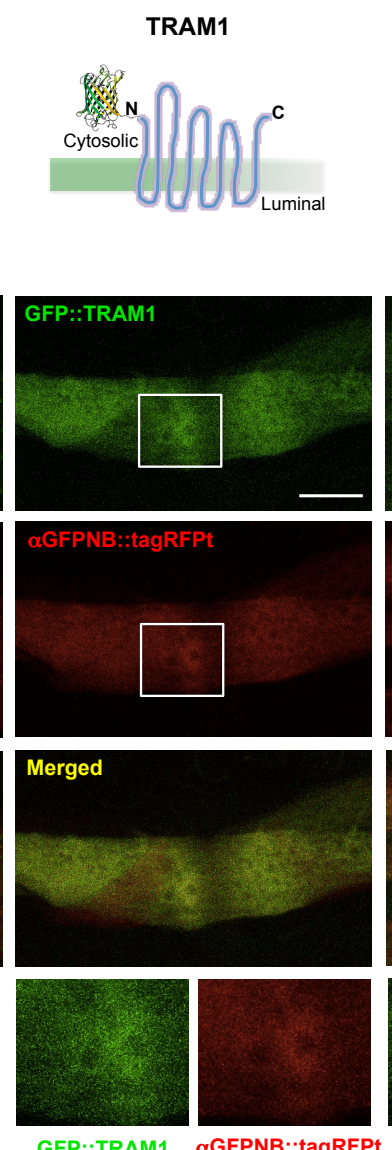

Colocalized
D

Cytochrome b5
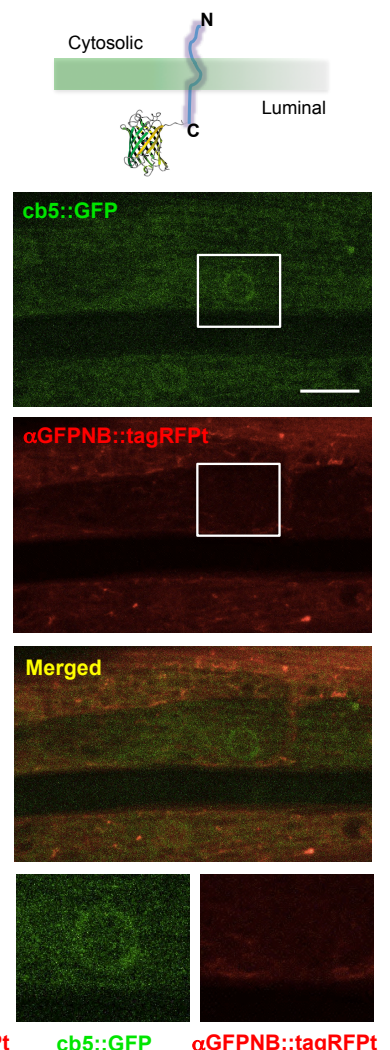

Not Colocalized

Figure VII.12 Specificity of the $\alpha$ GFPNB to the GFP fusion proteins in the Golgi and ER.

Schematic topologies of the organelle proteins which were fused to GFP or YFP in either cytosolic and/or luminal compartments are shown. The confocal images of the fusion proteins and $\alpha$ GFPNB are indicated to be functional, but spatially restricted in the lumen. The C-terminal lumen fusion proteins UNC-50, $\alpha$-Mannosidase II and Cytochrome b5 (cb5) did not colocalize with $\alpha$ GFPNB::tagRFPt (A,B and D), whereas the N-terminal cytosolic-fusion protein TRAM1 TRAM1 was shown to be colocalized as the complex GFP:: $\alpha$ GFPNB::tagRFPt (C). White boxes were magnified at the bottom of each image. Scale bars, $10 \mu \mathrm{m}$. 


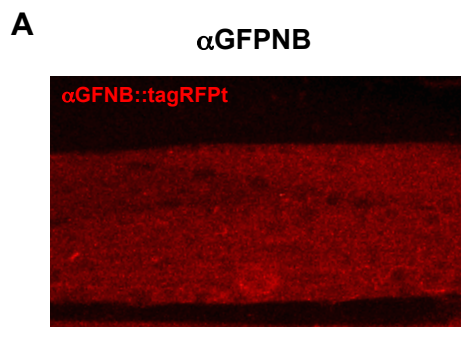

B
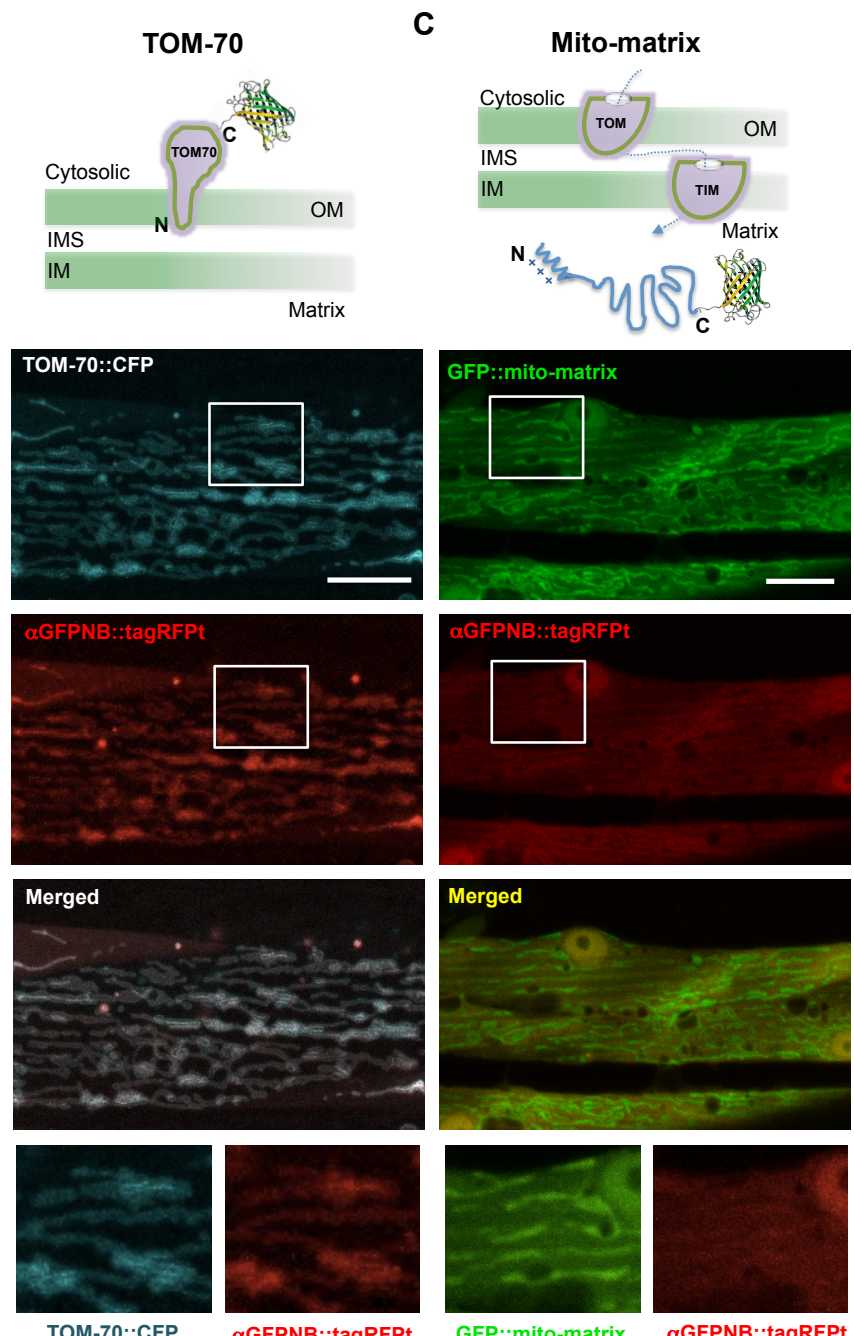

GFP::mito-matrix

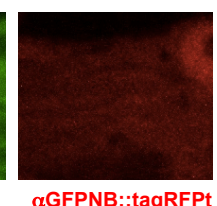

Colocalized

Not Colocalized

Figure VII.13 Specificity of the $\alpha$ GFPNB to the GFP/CFP fusion proteins in the mitochondria. As a control, $\alpha$ GFPNB::tagRFPt was expressed alone in C. elegans muscles, which appeared weak signal-intensities due to no antigen fusion proteins in cytosol (A). Schematic topologies of the mitochondria proteins TOM-70 and matrix-targeting signals using the stable transgenic line zcIs 14 [pmyo-3::GFP (mito-matrix)] are depicted in (B) and (C). As shown in confocal images, the $\alpha$ GFPNB::tagRFPt was shown to be the same images of the TOM$70:: \mathrm{CFP}$, indicating the $\alpha \mathrm{GFPNB}:: \operatorname{tagRFPt}$ was physically binding to the mitochondria outer- 
membrane fusion protein TOM-70::CFP in the cytosol (B). Mitochondria (mito) matrix-targeting import-signals (mito-matrix) are synthesized in cytosol and transferred into the matrix by cytosolic chaperones through the translocase machinery of TOM-TIM in the outer membrane (OM) and the inner membrane (IM), respectively (Tokatlidis et al., 2000; Wiedemann et al., 2004). The translocation of the cytosolic mito-matrix was normally shown in the GFP fusion proteins (GFP::mito-matrix), but not in the $\alpha$ GFPNB::tagRFPt (E) that was mostly cytosolic. In the merged image $(\mathrm{C})$, the mito-matrix was shown clearly not to be colocalized each other. White boxes were magnified at the bottom of each image. Scale bars, $10 \mu \mathrm{m}$.

A
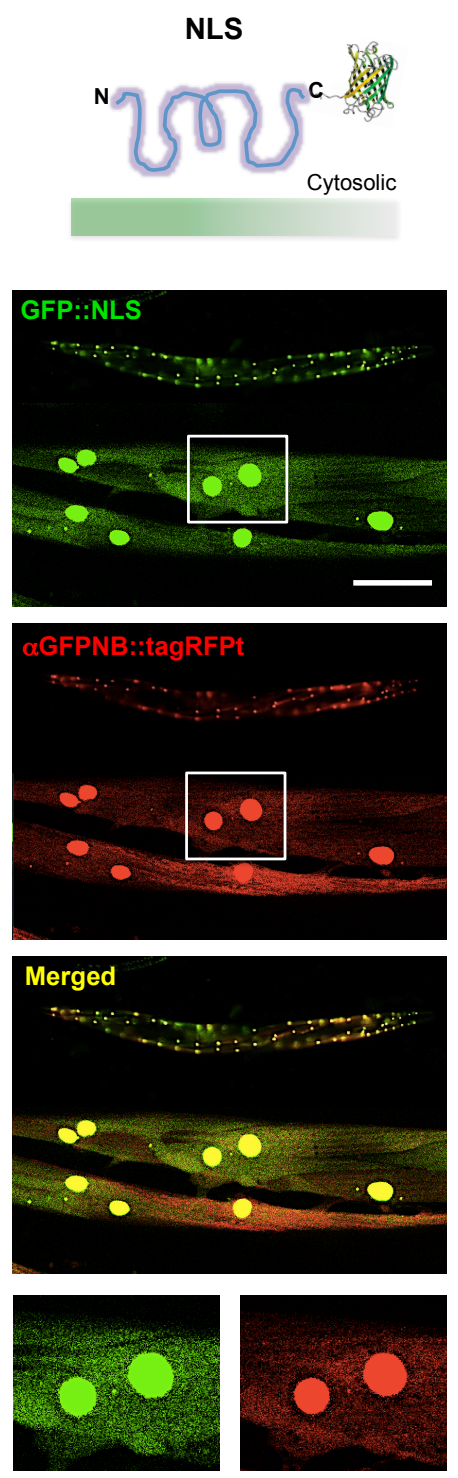

GFP::NLS

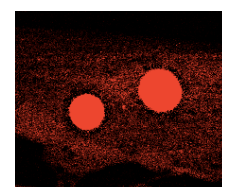

$\alpha$ GFPNB::tagRFPt

Colocalized
B
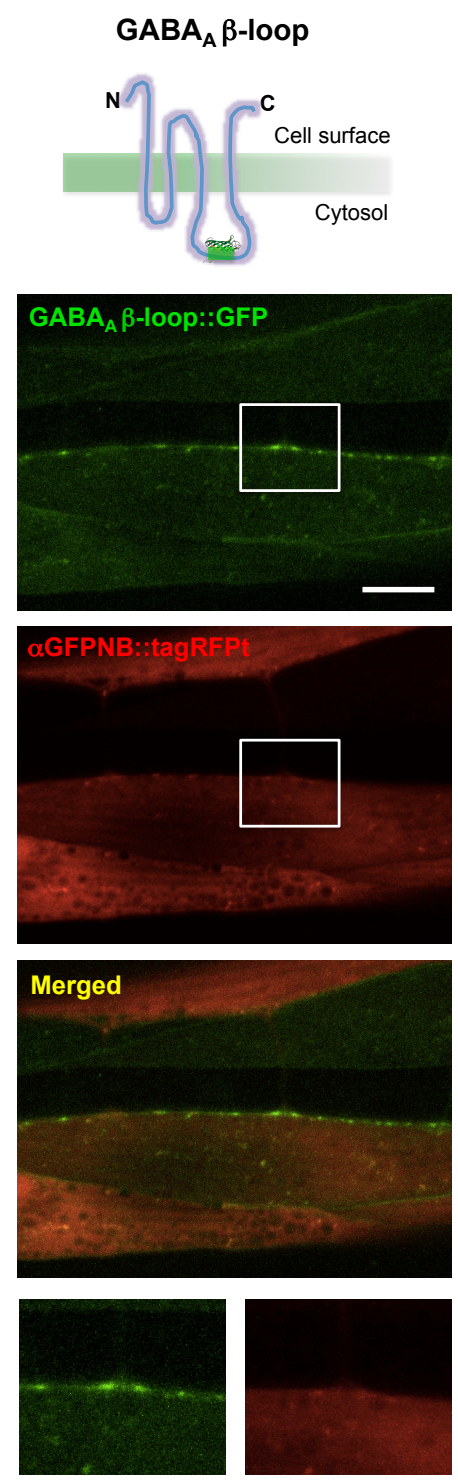

GABA $_{A}:$ :GFP $\quad \alpha$ GFPNB::tagRFPt

Not Colocalized 
Figure VII.14 Specificity of the $\alpha$ GFPNB to the GFP fusion proteins in the nucleus and NMJs. Schematic topologies of the fusion proteins nuclear localization signals (NLS) and a loop $\beta$-subunit of $\mathrm{GABA}_{\mathrm{A}}$ receptors encoded by $u n c-49$ in synapses at NMJs. In this study, we used

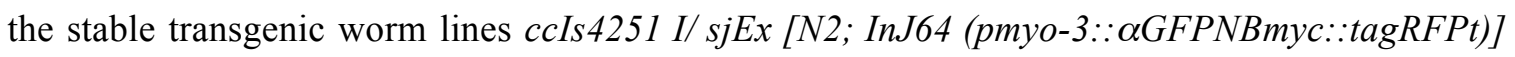
(A) and oxIs22 II; sjEx [N2; InJ64 (pmyo-3:: $\alpha$ GFPNBmyc::tagRFPt)] (B). The cytosolic NLS fusion proteins were shown to be colocalized with the $\alpha$ GFPNB::tagRFPt in the nucleus, indicating that the translocation of the NLS from the cytosol into the nucleus was functionally processed by nuclear transport such as importins that strongly binds to the NLS signals. The images of the dashed white boxes indicate magnified images of nucleus colocalized in muscles (A). At the synaptic area, a loop $\beta$-subunit of $\mathrm{GABA}_{\mathrm{A}}$ receptors was not detected by the $\alpha$ GFPNB::tagRFPt that was shown in the entire cytosol of muscle cells and segregated between the muscles (red) and receptors (green) expressed at the NMJs (B). White boxes were magnified at the bottom of each image. Scale bars, $20 \mu \mathrm{m}$ (A) and $10 \mu \mathrm{m}$ (B).

Taken together, the data indicate that the soluble $\alpha$ GFPNB complexes were functionally recruited to the GFP/CFP/YFP fusion proteins. As long as the GFP-tag is accessible from the cytosol, the $\alpha$ GFPNB-BirA* fusion protein should be able to be used as an affinity reagent for BioID. Except the NLS with the $\alpha$ GFPNB::tagRFPt that was transported into and be colocalized in the nucleus, the aGFPNB::tagRFPt was not able to bind GFP fusion proteins in the lumen of Golgi and ER, the translocation of mitochondria matrix-targeting signals into the matrix and a loop $\beta$-subunit of $\mathrm{GABA}_{\mathrm{A}}$ receptor at the NMJs in C. elegans. This suggests that the highly soluble $\alpha$ GFPNB complexes were spatially restricted to interact the antigen GFP fusion proteins in the lumen by the membrane barriers of the intracellular organelles ER-Golgi-endosomes. 


\section{DISCUSSION}

\subsection{Metabolic SILAC labeling of proteins relies on feeding periods and concentration of the bacteria SLE1 in C. elegans}

Intracellular protein trafficking requires highly dynamic interactions among proteins that occur differential abundance of proteome in certain internal/external biological conditions. To reveal the quantification and identification of the proteins in living cells, the metabolic isotope labeling by SILAC into the proteome is required for accurate quantitative analysis of differential proteomes based on mass spectrometry. In vivo metabolic labeling had held a new era in studying proteomics in versatile research fields in living cells and have been extended to apply complex living organisms (Findlay et al., 2008; Fredens et al., 2011; Gruhler et al., 2005; Krijgsveld et al., 2003; Kruger et al., 2008; Lahm and Langen, 2000; Larance et al., 2011; Oda et al., 1999; Ong et al., 2002).

Applying SILAC labeling $\left({ }^{15} \mathrm{~N}_{2-}{ }^{13} \mathrm{C}_{6}\right.$-lysine and ${ }^{15} \mathrm{~N}_{4-}{ }^{13} \mathrm{C}_{6}$-arginine) into the nematodes can be difficult due to incomplete incorporation of heavy amino acids into the whole proteomes in living organisms and arginine-to-proline conversion as well by proline biosynthetic pathways in $C$. elegans. By using a stable SILAC labeling method for $C$. elegans developed by the group of Lamond (Larance et al., 2011), the proline conversion can be negated by feeding E. coli SLE1 the ornithine transaminase encoded by orn-1 targeted RNAi feeding vector-containing strains during the growth in SILAC in vivo labeling processes (Larance et al., 2011). In this study, the metabolic isotope labeling by SILAC for accurate quantitative proteomics by referring the two experiments was established and the rates of metabolic incorporation were evaluated in several phased generations of $C$. elegans via feeding bacteria SLE1 (Fredens et al., 2011; Larance et al., 2011). As the results, we found that fully stable incorporation of the isotopes into proteomes was affected in feeding periods to be near-complete incorporation $( \pm 93 \%)$ that should be fed at least until F5-generation or more and be considered about the bacteria concentration (50-fold, $\mathrm{OD}_{600}$ of 1) for efficient in vivo labeling in C. elegans (Fig. VII.2). Why is the labeling efficiency not 100\%? In principle, it should be labeled with heavy isotopes to $100 \%$ but in practice the commercial sources $\left({ }^{13} \mathrm{C}_{6},{ }^{15} \mathrm{~N}_{2}\right.$, 
Cambridge Isotope Laboratories) assure isotope enrichment between 95\% and 98\% in cell cultures according to the group of Matthias Mann. In the case of C. elegans as a living organism, the labeling efficiency of SILAC was reported to $\sim 93 \%$ (Lys8, Arg10, 200) and $\sim 98 \%$ (Lys8) by the commercial isotope (Fredens et al., 2011; Larance et al., 2011). Based on the two references, we found the difference in molar amounts of both light and heavy-isotope (labeling efficiency of $\sim 93 \%$ from $0.2 \mathrm{mM}$ and $\sim 98 \%$ from 0.4 $\mathrm{mM}$ ), suggesting that in order to complete incorporation in C. elegans the isotope enrichment may affect the labeling efficiency in practice. In addition, Arg-to-Pro conversion through the biosynthetic pathway occurs in C. elegans unlike bacteria. As such, metabolic labeling of proteins in C. elegans should be applied in both light- and heavy-isotope to be normalized prior to mass ratio analysis on the peptide levels (Larance et al., 2011).

As an alternative way to incorporate isotope labeling instead of SILAC, the heavy nitrogen $\left({ }^{15} \mathrm{~N}\right)$ can be used in metabolic labeling to avoid the arginine-to-proline conversion in C. elegans (Gouw et al., 2010; Gouw et al., 2011; Krijgsveld et al., 2003). However, different amino acids have various numbers of the nitrogen atoms, unlike SILAC that labels the isotopes by essential amino acids as defined numbers in living cells, which makes ambiguous interpretation in the results of MS spectra that would miss identification of crucial peptides in the end (Ong et al., 2002).

In the case of the SILAC via the RNAi feeding vector-containing bacteria that could be reduced efficiency in knockdown by incomplete RNAi in the neurons of $C$. elegans as reported previously (Calixto et al., 2010), the gene targeted-RNAi would be affected in other protein expression compared to the control RNAi samples even though it appeared to be minimized (Larance et al., 2011). However, in SILAC labeling, the treatment of the RNAi via feeding bacteria should be applied in both heavy- and light-isotopes for executing control-SILAC and test-SILAC. Equal treatment of samples could consequently be negated in ratios and be considered as experimental contaminants in peptide pools identified, in which the proteome profiling results to be verified would also be normalized by sample replicates and reciprocal labeling arrangements (Larance et al., 2011). 
Therefore, using SILAC labeling, quantifying and identifying protein-protein interactions of transient proteins involved in intracellular dynamic trafficking would be a proper application in studying proteomics as well as our findings in metabolic incorporates of isotopes into the proteins would be fundamentally guide to the further applications based on various manipulations of neurogenetics in C. elegans.

\section{2 unc-50 mutants lead to protein abundance involved in the intracellular transport}

We have optimized MS-based quantitative proteomics by SILAC-isotope labeling into proteomes to investigate accurate protein quantification and identification in the functional organism C. elegans, in which unc-50 mutants lead to quantitative protein abundance in anterograde-promoting trafficking factors and actin-myosin cytoskeletonrelated scaffold factors that were significantly shown to be up-regulated at least in one replicate (Table VII.1B).

It is likely that the absence of the retrograde trafficking factor UNC-50 may cause disturbance in tight regulation that results in promoting endosome-mediated membrane dynamic factors that include GPB-1, UNC-18 and VPS-32.1 shown to be up-regulated in this study (McEwen and Kaplan, 2008; Thyagarajan et al., 2011), supporting that UNC50 localized at the Golgi-endosomal interface may be right place to regulate as a trafficking factor.

In addition, quantitative protein abundance of ACT-2 and NMY-2 in unc-50 mutants would be critical evidence to be involved in actin-myosin cytoskeleton motility as a trafficking regulator, supporting that the ARF-GEFs GEA1/2, a physical interacting partner of UNC-50/GMH1, are required to maintain cell polarity and the organization of the actin cytoskeleton in previous studies (Zakrzewska et al., 2003). Moreover, a weak physical interaction between GMH1 and Rho4 GTPase known as a regulator in the interaction of BNR1-HOF1 involved in cytoskeleton organization was reported by the group of Peyroche (Chantalat et al., 2003). Consequently, UNC-50 is likely to be involved in actin-myosin cytoskeleton motility through the ARF-GEFs-dependent trafficking at the Golgi-endosomal interface. 
As shown to be up regulated proteins in unc-50 mutants, a highly conserved histone transport protein NASP-2 in both replicates (Nabeel-Shah et al., 2014), a component of chromatin H2B1, and PBRM-1, a transcriptional regulator by chromatin remodeling, are also consistent with the study of the mammalian UNC-50 called UNCL that has been suggested as an inner nuclear membrane protein (INM) such as lamina-associated polypeptide-2 (LAP-2) that binds directly to chromatin, but not involving direct interaction with UNCL (Fitzgerald et al., 2000).

Based on the fold changes by SILAC ratios in unc-50 mutants, there are two-pair quantification results in transport factors. The anterograde and retrograde promoting factors were detected to be up and/or down-regulated. At the same time, the cytoskeletonrelated motilit actin-myosin factors versus dynein-mediated microtubule factors appeared to be up and down-regulated (Table VII.1). In particular, the BICD-1, which is required for the retrograde migration of Rab6-positive vesicles to the pericentrosomes and the Golgi-to-ER as a dynein-mediated transport along microtubules, was shown in both replicates to be down-regulated, suggesting that the retrograde secretory vesicles could be altered by the lack of the retrograde factors such as BICD-1 and UNC-50 (Matanis et al., 2002; Schlager et al., 2010; Terenzio and Schiavo, 2010).

As the other findings, the secretory yolk proteins VIT-3 and VIT-5 as lipid transport proteins that are secreted into the body cavity from the intestine and then taken up by oocytes of C. elegans gonad were quantitatively down-regulated in unc-50 mutants (Table VII.1D) (Kamath et al., 2003; Spieth and Blumenthal, 1985). As the corresponding results as initially observed, unc-50 mutants may cause secretion defects of MIG-17 from the muscles into the body cavity for distal tip cell migrations of gonads as well as a secreted signal sequence tagged-GFP (ssGFP) was observed to be trapped in the body wall muscles in the absence of UNC-50. In addition, the morphological analysis of the body wall muscles by HPF-EM in Chapter I revealed that the absence of UNC-50 causes unusual large vesicles (lipid-like) near mitochondria in the muscle belly. These results suggest that the lipid metabolisms in the unc-50 mutants might be unbalanced by the lipid binding precursors VIT-3 and VIT-5 that were shown to be down-regulated.

Based on the differential quantification of proteins, the fundamental role of UNC-50 is likely to be largely involved in transport, which could support all the results of Chapter I. 
As a conclusion, unc-50 mutants lead to enhanced forward trafficking dynamic by differential quantitative proteins up- and down-regulated in the intracellular transport.

\subsection{Specificity of the anti-GFP nanobodies based on the protein structure}

To identify binding partners of the transient protein UNC-50, we adapted rising proteomic technologies that provide accurate, stable and in vivo functional information. We found that the biotin protein ligase (BirA*, R118G) to apply SILAC-BioID for affinity purification prior to MS was functional for promiscuous in vivo labeling of UNC50 in all three strains gzIs20 (UNC-50::BirA*), gzIs21 (GFP::BirA*) and gzIs22 ( $\alpha$ GFPNB::BirA*) in C. elegans. From this, we revealed that endogenously biotinylated proteins in C. elegans were about $80 \mathrm{kDa}$ in size to be similar in yeast and humans, which would be informed and applied in functional studies including SILAC and affinity purifications in C. elegans.

As the strains $\alpha$ GFPNB and GFP that can be used in control-SILAC (Fig. VII.12), the $\alpha$ GFPNB complexes were evaluated in organelle specific markers (ER, Golgi, mitochondria, nucleus, and synapses of neuromuscular junctions) with GFP/YFP/CFP fusion proteins in C. elegans. We showed that highly soluble $\alpha$ GFPNB was specifically targeted into the organelle fusion proteins GFP/YFP/CFP using the form of QGFPNB::tagRFPt $(2.5 \mathrm{~nm},<40 \mathrm{kDa})$ in the cytoplasm (Rothbauer et al., 2008; Rothbauer et al., 2006), however the $\alpha$ GFPNB complexes was spatially restricted in the lumen by membrane barriers between the fusion proteins and $\alpha$ GFPNB::tagRFPt (Fig. VIII.1). 
A

Colocalized as GFP:: $\alpha$ GFPNB::tagRFPt

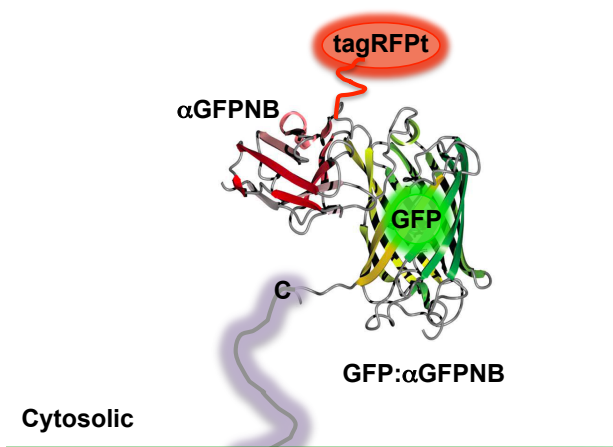

B

Restricted to be colocalized

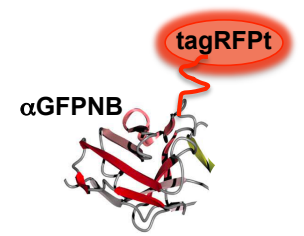

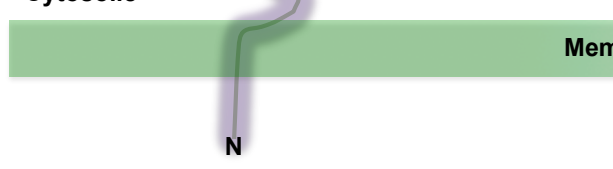

Membrane-barrier

Topology

Restricted to the lumen
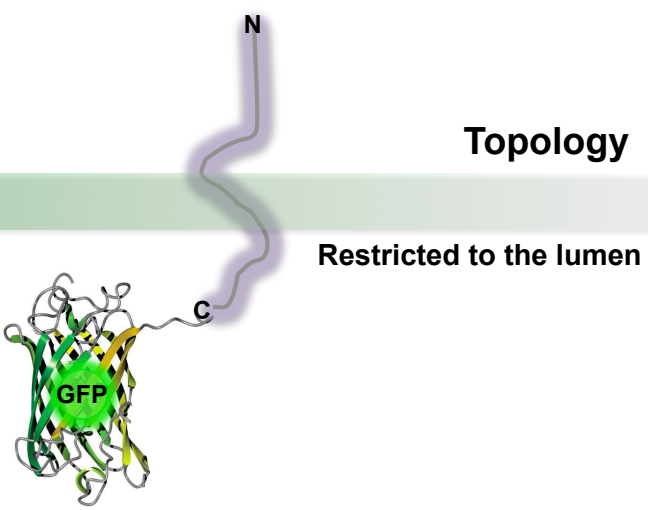

Figure VIII.1 Schematic specificity of $\alpha$ GFPNB complexes in the lumen and cytosol. The aGFPNB complexes can be functionally used in C. elegans. According to the labeling structure of the fusion proteins, it can be binding to the antigen GFP in the cytoplasm (A), whereas the GFP fusion proteins buried in the lumen are not conjugated with $\alpha$ GFPNB complexes by the membrane barrier (B).

In order to test whether translocation of mitochondria (mito) matrix-targeting importsignals (mito-matrix) from the cytosol into the matrix of mitochondria can be through to be functional by using the $\alpha$ GFPNB complexes, the GFP fusion protein of the mitomatrix signals was investigated. In this case, only the GFP fusion-mito signals were normally imported into the matrix but not with the aGFPNB complexes. Whereas the $\alpha$ GFPNB complexes with the TOM70 fusion protein as a cytosolic targeting tail in the outer membrane of mitochondria and NLS into the nucleus from the cytosol as the complexes with $\alpha$ GFPNB were shown to be functional and localized in C. elegans. In the NMJs, the $\alpha$ GFPNB complexes were mostly segregated into the muscle and a loop $\beta$ subunit of $\mathrm{GABA}_{\mathrm{A}}$ receptors at synapses (Fig. VII.11), indicating that the GFP fusion proteins and $\alpha$ GFPNB complexes may be spatially and structurally restricted by intracellular membrane barriers. 
These practical examples for functional labeling with the intracellular organelle proteins, translocation and synapses in C. elegans will provide possibilities of the structural manipulations to be functional and the fusion proteins with the aGFPNB complexes can also be used versatile applications in combination with biochemical, proteomic and functional studies as an endogenous epitope in living cells. 


\section{MATERIALS AND METHODS}

\subsection{Materials and Reagents}

All materials, reagents, oligonucleotides, plasmids, research equipments and strains (bacterial E. coli, yeast $S$. cerevisiae and nematode $C$. elegans strains) are listed and described in XI. Appendix.

\subsection{Methods in Molecular biology}

\subsubsection{Maintenance of bacterial strains}

All E. coli strains used in this study are listed in Table XI.1.1 with description. A single colony grown on a fresh LB-agar solid medium containing antibiotics if necessary was selected and overnight cultured in sterile LB medium with antibiotics (Appendix) at $37^{\circ} \mathrm{C}$ on a shaking incubator. A colony was streaked out on a fresh plate every other week.

\subsubsection{Molecular biology: PCR, Cloning and Transformation}

All constructs, primers for Polymerase Chain Reaction (PCR) and restriction enzymes used were described in the Appendix. Interested genes in C. elegans were amplified in genomic DNA and cDNA library (ProQuest, Invitrogen and/or RB1/2/3). PCR was conducted with a standard protocol as follows: if total volume is $25 \mu 1$, the PCR mixture contains $2.5 \mu \mathrm{l}$ of 10x PCR buffer (Stratagene, Invitrogen or Roche), $1 \mu \mathrm{l}$ of each primer $[10 \mu \mathrm{M}$ ], $0.5 \mu \mathrm{dNTPs}[12.5 \mathrm{mM}$ ], $0.5 \mu \mathrm{l}$ of Polymerase (Stratagene, Invitrogen or Roche for cloning and pTC for genotyping), DNA conc. 20 - $70 \mathrm{ng}$ as a template and double distilled $\mathrm{H}_{2} \mathrm{O}\left(\mathrm{ddH}_{2} \mathrm{O}\right)$ for the rest up to the total volume. A standard parameter of thermocycler was used for reactions as follows: $95^{\circ} \mathrm{C}$ for 2 minutes; 35 cycles $\left[95^{\circ} \mathrm{C}\right.$ for 20 seconds to $(\boldsymbol{x})^{\circ} \mathrm{C}$ for 20 seconds to $72^{\circ} \mathrm{C}$ for $(\boldsymbol{x})$ minutes]; $72^{\circ} \mathrm{C}$ for 3 minutes; $4{ }^{\circ} \mathrm{C}$. The value of $\boldsymbol{x}$ was dependent on each primer pair used for PCR reaction. Otherwise the conditions used in this study were shown in Table XI.2.2 and 2.3 (Appendix). PCR 
products were confirmed on 1\% agarose gels (Table XI.5.1).

Molecular cloning was performed using classical techniques (Sambrook and Russell, 2001). In this study, restricted digestion and ligation of constructs as a typical cloning strategy were performed with restriction enzymes, T4 DNA ligase and proper buffers as manufacturer's instructions (Fermentas and New England Biolabs). As for the enzyme digestion, total $20 \mu \mathrm{l}$ of volume contains $2 \mu \mathrm{l}$ of $10 \mathrm{x}$ digestion buffer, $0.5 \mu \mathrm{l}$ of each restriction enzyme, $1 \mu \mathrm{g}$ of DNA and $\mathrm{ddH}_{2} \mathrm{O}$ for the rest volume. The reaction of digestion was kept at $37^{\circ} \mathrm{C}$ for $3-4$ hours. Then after, the reaction was confirmed on $1 \%$ agarose gels with 1x TBE buffer (Appendix). The ligation reaction of the fragments digested by restriction enzymes was carried out in total $20 \mu 1$ volume containing $2 \mu 1$ of 10x ligation buffer, $1 \mu$ of T4 ligase, 40 ng DNA (1:3 ratios of vector and insert) and $\mathrm{ddH}_{2} \mathrm{O}$ for the rest volume. It was reacted at $4{ }^{\circ} \mathrm{C}$ for overnight and transformed into 100 $\mu 1$ of DH5 $\alpha$ competent cells by using heat-shock transformation on a heat-block at $42^{\circ} \mathrm{C}$ for 45 seconds and put it back on ice for 5 minutes. The transformed cells were recovered in $900 \mu \mathrm{l}$ of LB medium (Appendix) and incubated on a shaker at $37^{\circ} \mathrm{C}$. After $45-60$ minutes, the cells were collected and plated on LB-agar plates containing ampicillin (Appendix). A single colony of the plate overnight-incubated at $37^{\circ} \mathrm{C}$ was selected and inoculated in $5 \mathrm{ml} \mathrm{LB}$ media containing ampicillin for overnight. Plasmid DNAs were isolated by Mini-prep kit (Invitrogen) as manufacturer's instructions. Positive clones confirmed by restriction enzymes were verified by sequencing.

\subsubsection{Agarose gel electrophoresis}

Amplified PCR reactions and digested plasmids to be verified were analyzed in agarose gel electrophoresis. $1 \%$ of ethidium bromide was added $0.1 \mu \mathrm{l} / \mathrm{ml}$ into the melted agarose in $1 \mathrm{x}$ TBE buffer (Appendix) by microwave and poured on the gel caster for electrophoresis. PCR products and the reaction samples after enzyme digestion were sizeanalyzed on $1 \%$ agarose gels with TBE buffer. Before loading on gel pockets, the samples were mixed with 6x DNA loading buffer (Fermentas) and separated on gel in 1x TBE buffer with $1 \mathrm{~kb}$ DNA ladder (Fermentas) at $130 \mathrm{~V}$ for 40 minutes. In order to do restriction digestion and ligation as a cloning strategy, corresponding bands were 
extracted by cut under ultraviolet light and purified using commercial gel extraction kits (Appendix).

\subsubsection{Sequencing}

All genes in plasmid constructs whether they have correctly sequenced as designed in this study were confirmed by sequencing company (GATC Biotech) before use or after the cloning. Sequencing primers and oligonucleotides used were placed in the Appendix.

\subsection{Methods used in the nematode Caenorhabditis elegans}

\subsubsection{Maintenance of strains}

All strains used in C. elegans were maintained at $20^{\circ} \mathrm{C}$ on standard nematode growth medium (NGM) plates seeded with E. coli OP50 as described previously (Brenner, 1974). All mutant strains were outcrossed at least three times before use. Some nematode strains used in this study were provided by the CGC, which is funded by the NIH NCRR. arfrpl (tm2779) was supplied by the NBRP (Tokyo Women's Medical University School of Medicine). unc-50 (ok1847) was provided by Gene Knockout Project at Oklahoma Medical Research Foundation (OMRF), which is part of the international C. elegans Gene Knockout Consortium.

\subsubsection{Crossing worms}

To select homozygous mutants [e.g. unc-50 (x47)], the worms were outcrossed with males of a marker strain ccIs4251 [pmyo-3::nls-gfp; pmyo-3::mt-gfp]; him-8 (e1489) (Fire et al., 1998) and creating deletion mutants of stable integration lines [e.g. pwIs50 [lmp-1::GFP] (Treusch et al., 2004) and arIs37 [pmyo-3::ssGFP] (Fares and Greenwald, 2001)] was used by crossing steps between the worms as follows. The males of $10-15$ worms were put together with hermaphrodites of $3-4$ young adults on bacteria OP50 seeded NGM plates. After 48 hours, all adults were removed out of the plate and their F1

progeny were isolated on a single plate for laying eggs of F2 progeny. Corresponding markers, fluorescent-expressed or drug resistance phenotypes, of the progeny were tested 
to isolate homozygous F2 generation, which were confirmed by microscopy, worm PCR and chemical compound sensitivity for isolating homozygous worms.

\subsubsection{Worm PCR templates}

From a single to tens animals, worm strains were lysed in worm lysis buffer containing proteinase $\mathrm{K}$ (Appendix) before freezing at $-80^{\circ} \mathrm{C}$ for 20 minutes. The frozen worm body was initially ice crystalized in the lysis buffer that allows to be lysate and digest proteins by proteinase $\mathrm{K}$ at $65^{\circ} \mathrm{C}$ for 1 hour; $95^{\circ} \mathrm{C}$ for 20 minutes to inactivate proteinase $\mathrm{K}$ for PCR reaction with DNA polymerase. The final worm lysate was used as a template for Worm PCR to confirm the gene-depleted homozygous or heterozygous strains through the PCR steps that has been previously described in IX.2.2. The reaction of worm PCR was verified on $1 \%$ agarose gels. All the primers deletion alleles used in this study were listed in the Appendix.

\subsubsection{Molecular Cloning Strategy}

In this study, all cloned genes and constructs that were amplified from a cDNA library were tabulated in the Appendix. The amplified genes by PCR were cloned by classical cloning methods as previously described in IX.2.2 into pBluescript SKII (Stratagene) and/or $p G E M T$ cloning vector (Promega). To exam the expression pattern of organelle specific markers (e.g. APT-9 at trans-Golgi networks) in body wall muscle cells, the genes were subcloned with a corresponding construct by matching restriction enzyme sites. As a backbone plasmid in this study, pPD115.62 (Pmyo-3::gfp) was used for expression of muscle cells. Fluorescent-labeled genes used in this study were subcloned as pairs of AgeI/NotI fragment, AgeI/XhoI fragment and NotI/BamHI fragment with GFP, mCherry, mYFP, TagRFPt and TagRFP into pPD115.62 vector bone containing the myo3 promoter. All genes cloned and subcloned into the destined vectors in this study were described in the Appendix.

\subsubsection{Transgenic lines and chromosomal integrated strains}

The transgenic worm lines performed by microinjection methods were typically generated as previously described (Mello and Fire, 1995). Specific cells and tissues in 
genetic mosaics can be observed to get insight the cellular specificity of induced genes by microinjection into gonads in C. elegans. The mutants of unc-50 that have a levamisoleresistant phenotype were tested to rescue worms by inducing unc-50 that has been fused with a fluorescent or enzymatic indicator in muscle cells. Because, in genetic mosaics, a single fertilized egg after microinjection also goes through typical cell division such as meiosis and mitosis by which different cells carrying extrachromosomal precursors have different genotypes in an individual worm (Sulston et al., 1983). Thus, targeted muscle cells for studying the intracellular phenotypes of gene function can be analyzed by microscopy and functional confirmation (e.g. levamisole resistance) of the interest protein through transgenic worms. In order to generate transgenic lines, young adult hermaphrodites were used for microinjection, and heritable transgenic lines were obtained from F2 offspring that contains visual positive markers. In this study, $p t t x-3:: g f p$ and pRF4 (rol-6 (su1006)) were used as co-injection markers in $20 \mathrm{ng} / \mu \mathrm{l}$ and $40 \mathrm{ng} / \mu \mathrm{l}$, respectively and the total concentration of DNA mixtures were added up to $100 \mathrm{ng} / \mu \mathrm{l}$ by implementing pBlueScript SKII. All the transgenic lines by microinjection in this study were also listed in the Appendix. The unc-50::birA* [InJ60], GFP::birA* [InJ61] and GFPNBmyc::birA* [InJ62] extrachromosomal arrays, respective gzIs20, gzIs21 and gzIs 22 were chromosomally integrated by gamma irradiation using a ${ }^{137} \mathrm{Cs}$ source (RX30/50, Gravatom Industries). The integrated strains were outcrossed three times with $\mathrm{N} 2$ males.

\subsubsection{Movement assays}

In order to confirm functional rescue in transgenic animals, the locomotion behavior of worms were observed on the non-seeded E. coli OP50 NGM plate. For this assay, young worms non-starved were transferred and initially adjusted on the plate for 60 minutes. The sinusoidal wave along the worm body (corresponding to a whole $360^{\circ}$ sine wave) was counted over a 1 or 3-minuite time interval and twenty to forty worms were analyzed.

\subsubsection{Pharmacological assays}


Cholinergic neurotransmission in $C$. elegans can be analyzed with pharmacological materials, levamisole and aldicarb (Sigma Aldrich). In order to identify homozygous unc50 mutants, worms were analyzed on bacteria OP50 seeded-plates containing $1 \mathrm{mM}$ levamisole as described previously (Lewis et al., 1980). Ten to thirty worms per strain were transferred onto the levamisole (Lev) plates and assessed by knocking the plate or touching the head to confirm paralyzed worms. After one hour of exposure, the percentage of paralyzed worms was quantified or just confirmed by their paralyzed phenotypes on the Lev plate. Drug aldicarb resistance was also observed to test cholinergic transmission in unc-50 mutants. The plates containing $2 \mathrm{mM}$ aldicarb were prepared and conducted as previously described (Mahoney et al., 2006). The worm response on the plate of aldicarb was analyzed and quantified at the indicated time points.

\subsubsection{TR-BSA endocytosis assays}

Texas-red conjugated to bovine serum albumin (TR-BSA, $1 \mathrm{mg} / \mathrm{ml}$ ) was used for analyzing endocytosis dynamics as a bulk fluid-phase internalization marker within coelomocytes of young adults which were crossed with the integrated strain bIs 34 [prme8::rme-8-GFP; rol-6 (su1006)] expressing GFP fusions of RME-8 as a positive endosome marker in both wild type and unc-50 (x47) mutants. In order to see the liquid flowing from pseudocoelomic body cavity into coelomocytes, it was injected into the body cavity in the pharyngeal region as described previously (Zhang et al., 2001). As the time points 10, 30 and 50 minutes after injecting at least into five worms per each time, the uptake of TR-BSA was observed by confocal microscopy and the images taken by line-averaged at the middle of the cells were analyzed using ImageJ software (National Institute of Health, USA).

\subsubsection{Confocal microscopy and imaging}

All microscope models used in this study are listed in Table XI.6.1. To analyze the expression of various proteins on body wall muscles of young adults C. elegans, inverted confocal microscopy were applied for analysis of immobilized worms with $50 \mathrm{mM}$ Sodium azide on $2 \%$ agarose pads. The images were observed and taken using $63 \mathrm{x}$ objective with oil immersion at $20^{\circ} \mathrm{C}$. Stack images were obtained for maximum intensity 
projections by operating the integrated confocal softwares (SP2-Leica in ENI, Göttingen, SP8-Leica in ZBSA, Freiburg and LSM510-Zeiss in ZBSA, Freiburg). By using confocal microscopy, sub-cellular localization and co-localization of proteins were assessed with intracellular organelle specific markers labeled-fluorescence co-expressed in body wall muscles. In order to analyze the relative quantification, the maximum intensity projections were taken by the confocal software (SP series, Leica) and adjusted by threshold to be quantified using ImageJ software (National Institute of Health, USA). Measured values were normalized to wild type and the relative quantification difference was decided by p-value less than 0.05 , Student's $t$ test. The imaging of bright-field observation was done with objective lenses ( $2 \mathrm{x}$ to $100 \mathrm{x}$ ) of inverted fluorescence phasecontrast microscope BZ-9000 (Keyence).

\subsubsection{Western blots}

Worms washed in M9 buffer or PBS (Appendix) were pelleted prior to be frozen and lyzed by sonicator in homogenization buffer (20 mM HEPES pH 7.4; $10 \mathrm{mM} \mathrm{KCl} ; 1 \mathrm{mM}$ EDTA; $400 \mu \mathrm{M}$ Pefabloc protease, Roche) or protease/phosphatase inhibitor cocktails (Complete protease inhibitor mini-tablets and PhosStop, Roche) with $5 \mathrm{mM} \mathrm{N}$ Ethylmaleimide (NEM, Sigma) in ice-cold PBS before freezing in liquid nitrogen. Worm lysates were boiled at $95^{\circ} \mathrm{C}$ for $5-10$ minutes in $4 x$ SDS loading buffer (Appendix) and separated on $10-15 \%$ SDS-PAGE gels (Appendix) in SDS running buffer (Appendix) at $150 \mathrm{~V}$ for 60 - 90 minutes using BioRad Mini-PROTEAN. By using Semi-Dry-Blotter, the proteins were transferred to a nitrocellulose membrane (Protran, Whatman) with the blot-buffer (Appendix) at $100 \mathrm{~mA}$ for 60 minutes and the membrane was 30-minute blocked on a shaking platform in the blocking solution (Appendix) before treating the primary antibody (Appendix) overnight at $4^{\circ} \mathrm{C}$. The membrane was then washed three times with TBST washing solution (Tris-buffered saline containing $0.1 \%(\mathrm{v} / \mathrm{v})$ Tween 20) and horseradish peroxidase (HRP)-conjugated secondary antibody (Jacksons Laboratory, Bar Harbor, Maine, USA) was incubated for 50 minutes at RT. The blot-membrane was developed using the developing solution (Table XI.5.1, Appendix) and the corresponding protein bands against the antibodies were detected using FujiFilm LAS 3000. The quantification of bands was analyzed by ImageJ software (National Institutes of Health). 
To analyze the quantification of whole proteins or protein-protein interactions (PPI) by mass spectrometry, the solubilized proteins with 4x SDS loading buffer were fragmented on $10 \%$ SDS or $4-15 \%$ Mini-PROTEAN ${ }^{\circledR}$ TGX $^{\text {TM }}$ Gel (Bio-rad) after the boiling steps. The gels were stained with Coomassie Brilliant Blue R250 stain solutions (Appendix) for 30 minutes and then destained with Coomassie destaining buffer (Appendix) until protein bands showed up on the gels.

\subsubsection{High-pressure freeze electron microscopy (HPF-EM)}

Bacteria OP50 were filled onto a $100 \mu \mathrm{m}$ deep aluminium platelet and twenty young adults were put into the chamber to be frozen immediately using a BalTec HPM 10. Then after, freezing substitution was performed with a Leica AFS2 as follows: Incubating at $90{ }^{\circ} \mathrm{C}$ for 100 hours in $0.1 \%$ tannicacid, $7 \mathrm{~h}$ in $2 \%$ Osmium tetroxide $\left(\mathrm{OsO}_{4}\right)$, and keeping at $-20{ }^{\circ} \mathrm{C}$ for 16 hours in $2 \% \mathrm{OsO}_{4}$. Embedding in EPON at room temperature (Rostaing et al., 2004). $50 \mathrm{~nm}$ sections were mounted on copper grid-slots and placed for ten minutes on drops of $4 \%(\mathrm{w} / \mathrm{v})$ uranyl acetate in $75 \%$ methanol and washed in $\mathrm{ddH}_{2} \mathrm{O}$. After drying, the grid-slots were placed on lead citrate (Reynolds, 1963) in a $\mathrm{CO}_{2}$-free chamber for two minutes before rinsed in $\mathrm{ddH}_{2} \mathrm{O}$. For the morphological analysis of the body wall muscles, young adults worms in wild type were compared with unc-50 (x47) mutant animals. In this study, HPF-EM was conducted by Oliver Kretz.

\subsubsection{Stable isotope labeling by amino acids in C. elegans (SILAC)}

In order to be isotopically labeled amino acids in C. elegans, wild type and unc-50 mutants (x47) were maintained at $20^{\circ} \mathrm{C}$ as previously described (Brenner, 1974). For metabolically labeling amino acids into the worm, hermaphrodites having eggs can be egg-released by egg preparations (egg prep.) in the solution $500 \mu \mathrm{l} 5 \mathrm{M} \mathrm{NaOH}$ and $1 \mathrm{ml}$ bleach solution (5\% solution of sodium hypochlorite) in $3 \mathrm{ml}$ M9 buffer (Appendix) by vortexing, centrifugation and washing in $\mathrm{ddH}_{2} \mathrm{O}$ (http://wormbook.org/), otherwise the worms were grown on nematode growth medium without nitrogen source (NGM-N) agarose plates (Appendix) after removing bacteria contaminants with the bleaching solutions. The worm strains were then egg-laying on the NGM-N and the larval stage (L1) 5 - 10 worms were transferred to the seeded plates of E. coli SLE1 strains, 
expressing the orn- 1 targeted RNAi feeding construct (Larance et al., 2011), which were grown in M9 minimal medium (M9MM) containing each $40 \mu \mathrm{g} / \mathrm{ml}(220 \mathrm{nM})$ of light (L$\mathrm{KR} ;{ }^{14} \mathrm{~N}_{2-}{ }^{12} \mathrm{C}_{6}$-lysine and ${ }^{14} \mathrm{~N}_{4}{ }^{12} \mathrm{C}_{6}$-arginine) or heavy (H-KR; ${ }^{15} \mathrm{~N}_{2}-{ }^{13} \mathrm{C}_{6}$-lysine and ${ }^{15} \mathrm{~N}_{4}$ ${ }^{13} \mathrm{C}_{6}$-arginine) amino acids (L-lysine and L-arginine), respectively. To prepare the bacteria strains for seeding on NGM-N, the SLE1 strains were streaked out on LB plates from a frozen stock and one colony was grown on the LB containing Amp ${ }^{\mathrm{r}}(100 \mu \mathrm{g} / \mathrm{ml})$ and $\operatorname{Tet}^{\mathrm{r}}(12.5 \mu \mathrm{g} / \mathrm{ml})$ plates at $37^{\circ} \mathrm{C}$ overnight. A single colony was incubated into L-KR or H-KR M9MM containing $1 \mu \mathrm{g} / \mathrm{ml}$ carbenicillin and $1 \mathrm{mM}$ IPTG for expressing the ornithine transaminase RNAi feeding vector to prevent arginine to proline conversion through biosynthetic urea cycle (Bicho et al., 2010). The worms to apply SILAC labeling for proteomics were then maintained on the SLE1 lawn pads which were concentrated to be 10 to 50 -fold in $\mathrm{OD}_{600}=1$ seeded on NGM-N agarose plates.

\subsubsection{Worm stocks}

To prepare C. elegans stocks, the freezing and recovery steps were performed as described previously (Brenner, 1974). Six worm plates (diameter of $9 \mathrm{~mm}$ ) were used to collect worms by flushing the plate using $\mathrm{S}$ buffer (Appendix) by centrifugation at 800 rpm for 10 minutes. $3 \mathrm{ml}$ worm freezing solutions ( $\mathrm{S}$ buffer $+30 \%$ glycerol) were added into the collected worms $3 \mathrm{ml}$ in $\mathrm{S}$ buffer and mixed to be aliquoted into cryovials ( $1 \mathrm{ml}$ per one cryovial). All six cryovials as worm stocks were kept at $-80^{\circ} \mathrm{C}$ and one of the worm stocks was used to test whether the freezing worms would be able to recover properly on a seeded NGM plate after thawing at RT.

\subsubsection{Integration lines by gamma irradiation of $C$. elegans}

L4-stage worms of extrachromosomal transgenic-lines Ex:InJ60, Ex:InJ61 and Ex:InJ62 (Table XII.3.4) were prepared to integrate extrachromosomal transgenes by gamma irradiation in C. elegans. The integration protocols used in this study were accepted and modified from Peg MacMorris and Ji Ying Sze (http://wormbook.org/). Total forty rolling worms per independent extachromasomal line for the integration were transferred onto NGM OP50-seeded plates and irradiated with 4000 Rads of gamma rays for 10 minutes from a 137Cesium source. The four to five irradiated L4 transgenic worms were 
transferred onto $6 \mathrm{~cm}$ plates and incubated at $20^{\circ} \mathrm{C}$ for two weeks. Among the plates after $48-72$ hours, one was used for quick isolation of the irradiated lines while other plates were being starved. If there was no successful finding, the starved worm plates were chunk and the picking worms after 24 - 36 hours. In this study, gzIs20/01, gzIs21/01, gzIs21/02 and gzIs21/03 and gzIs22/01 were successfully integrated by gamma irradiation. In order to label amino acids by SILAC, the integrated lines were outcrossed three times with N2 wild type worms. Each location on chromosomes of the integrated lines and whole genome sequences need to be confirmed by chromosomal mapping and sequencing information.

\subsection{Methods used in the yeast Saccharomyces cerevisiae}

\subsubsection{Molecular biology and strains used in yeast}

General molecular biology and basic methodology for yeast, Saccharomyces cerevisiae, strains used in this study were performed as described by Current Protocols in Molecular Biology (Ausubel et al., 1997). All strains and plasmids used in this study are listed in the Appendix.

\subsubsection{Yeast growth phenotypes}

Yeast cells were grown in synthetic complete (SC) or selective media lacking uracil (SCURA) for overnight at $30^{\circ} \mathrm{C}$ and normalized to an $\mathrm{OD}_{600}$ of 0.2 for six serial dilutions on testing plates. As for yeast growth phenotypes, Ferrozine ( $3 \mathrm{mM}$ and $1 \mathrm{mM}$ to $0.25 \mathrm{mM}$ ), Batho Phenantroline diSulfate (BPS) $(40 \mu \mathrm{M}), 2 \%$ glycerol/lactate and rapamycin (4 to $0.5 \mathrm{nM}$ ) plates were used on starvation and iron-limited conditions. Cells were then allowed to grow on testing plates containing chemical elements after spotting for incubation at $30^{\circ} \mathrm{C}$ for 3 days. Yeast growth phenotypes were analyzed after images were acquired. All chemicals, media and plates used in this study are shown in the supplementary materials (Appendix)

\subsubsection{Transformation of yeast cells}


Yeast cells were transformed using the standard lithium acetate (LiAc) protocol as previously described (Gietz et al., 1992). Cells cultured for overnight were inoculated $1: 10$ to an $\mathrm{OD}_{600}$ of 0.2 in fresh media and grown at $30^{\circ} \mathrm{C}$ on a shaking incubator for $\log$ phase yeast cultures. Cell pellets were then harvested and washed in $1 \mathrm{ml} \mathrm{ddH}_{2} \mathrm{O}$ for 5 minutes at $2000 \mathrm{rpm}$ before resuspended in $200 \mu \mathrm{l}$ of a lithium acetate mix solution (1:1:8) with $1.2 \mathrm{ml}$ of a polyethylene glycol (PEG) mix (8:1:1) using the following protocol: 10x TE (0.5 M Tris-HCl pH 7.5, $10 \mathrm{mM}$ EDTA), 10x LiAc (1 M LiAc pH 7.5), 50\% PEG (w/v) 3350 in $\mathrm{ddH}_{2} \mathrm{O}$, a LiAc mix solution (1:1:8, e.g. $1 \mathrm{ml}$ 10x TE, $1 \mathrm{ml}$ 10x

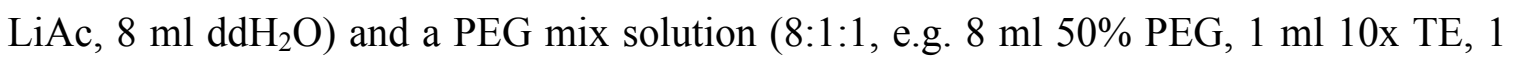
$\mathrm{ml}$ 10x LiAc). $18 \mu 1$ of carrier DNA (salmon herring sperm DNA, $10 \mathrm{mg} / \mathrm{ml}$ in Tris-HCl $\mathrm{pH}$ 8.0, 1 mM EDTA) heat-denatured in boiled water using microwave were added to each type of plasmid DNA $(0.5 \mu \mathrm{g})$ while preparing the mix solutions as described above. Yeast cells treated in the LiAc solution were together in $1.5 \mathrm{ml}$ tube with carrier DNA, each plasmid and the PEG mix solution before heat shock for one hour at $42^{\circ} \mathrm{C}$. Then the treated cells were harvested by centrifugation for 3 minutes at 4,000 rpm and resolved in $100 \mu 1 \mathrm{ddH}_{2} \mathrm{O}$. In case of the strains of the knockout-gmhl, they were inoculated in YPAD media with clonat (nourseothricin, final conc. $100 \mu \mathrm{g} / \mathrm{ml}$ ) for 30 minutes at $30^{\circ} \mathrm{C}$ before spreading them onto selective solid media. Testing plates were incubated for $3-4$ days at $30^{\circ} \mathrm{C}$ until colonies appear.

\subsubsection{Creating of knockout-gmh1 strains by PCR-based deletion cassettes}

Yeast strains used in this study were described in XI. Appendix and provided from the groups of Stefan Eimer, Blanche Schwappach and the EUROSCARF collection. Gmh1 deletion strain, $\Delta g m h 1:: \mathrm{Nat}^{\mathrm{R}}$, was used to generate the strain lacking GMH1 in the following strains, Anp1-RFP::Kan ${ }^{\mathrm{R}}, \quad$ Chc1-RFP::Kan ${ }^{\mathrm{R}}, \quad \operatorname{Snf7}-\mathrm{RFP}:: \operatorname{Kan}^{\mathrm{R}}$ and spep4:: $\mathrm{Kan}^{\mathrm{R}}$, by a PCR-mediated homologous recombination approach (Goldstein and McCusker, 1999; Wach et al., 1994). PCR was carried out in a solution containing $5 \mu 1$ Expand 10x PCR buffer (Roche), 2.5 units Expand DNA polymerase (Roche), $0.2 \mathrm{mM}$ dNTPs, $0.4 \mu \mathrm{M}$ of the appropriate forward oGQ2785 (GTG AAG TAC GAT CTA AAA CTG TTG) and reverse oGQ2786 (ACG ACC AGG AAC ACA CCA TTA) primer and DNA templates which genomic DNAs of $\Delta g m h 1:: \mathrm{Nat}^{\mathrm{R}}$ were used. The reaction 
conditions were as follows: initial denaturation at $95^{\circ} \mathrm{C}$ for 3 minutes, 35 cycles at $95^{\circ} \mathrm{C}$ for $20 \mathrm{~s}, 51^{\circ} \mathrm{C}$ for 1 minute $30 \mathrm{sec}$ and $72^{\circ} \mathrm{C}$ for 1 minute with a final extension at $72^{\circ} \mathrm{C}$ for 5 minutes. PCR products were separated and analyzed on a $1 \%$ agarose gel with SafeView ${ }^{\text {TM }}$ Nucleic acid stain (Applied Biological Materials Inc.). Fragments of the $\Delta g m h 1$ were cut from the gel for purification with High Pure PCR Product Purification Kit (Roche) according to the manufacturer's instructions and transformed into yeast using the lithium acetate protocol described as above. Colonies in which the destined one had been knockout-Gmh1 were selected on solid YPAD medium containing clonat (nourseothricin) as a resistant marker and confirmed by PCR using another primer forward oGQ617 (GGG ACT AGT TCT AGA ATG TCA TAT TTG CCG ACT TAT TCC) and reverse oGQ618 (GGG GTC GAC GGA TC C TTA ATA ATT GTA AAA GCT TAA TTT CTT GGA) for checking deletion of gmhl in the strain after transformation.

\subsubsection{Purification of genomic DNA from yeast cells}

$1.5 \mathrm{ml}$ of yeast culture was incubated for overnight in the appropriate selective yeast medium. Plasmid DNA was purified using the YeaStar ${ }^{\mathrm{TM}}$ Genomic DNA Kit (Zymo Research) according to the manufacturer's instructions. Cell lysis is based on digestion of the cell wall using $5 \mu$ l of R-Zymolyase (Zymo Research). DNA was then purified on a affinity column of the kit.

\subsubsection{Western blotting of yeast cellular lysates}

Yeast strains were overnight cultured in $2 \mathrm{ml}$ selective media at $30^{\circ} \mathrm{C}$ and measured cell growth at $\mathrm{OD}_{600}$ for equal loading samples. Each $500 \mu \mathrm{l}$ of the cultured cells was harvested by low speed centrifugation and then resuspended by $200 \mu \mathrm{l}$ of $0.1 \mathrm{M} \mathrm{NaOH}$ at room temperature for total cellular lysates (Yaffe and Schatz, 1984). After 10 minutes, samples were harvested and resolved by 1x loading buffer of SDS-PAGE containing 100 mM DTT as equivalent to $\mathrm{OD}_{600}$ of 0.01 unit per sample. Before loading on the pocket of $10 \%$ gel of SDS-PAGE, samples were spun down simply by centrifugation and then 10

$\mu 1$ of each sample was used for running on the gel in 1x SDS running buffer and transferred to nitrocellulose membranes in $1 \mathrm{x}$ transfer buffer for one and half an hour at 
60 voltage. Membranes were washed in blocking solution (1x TBS, 5\% milk powder, $0.02 \%$ IGEPAL CA-630) for 30 minutes after checking the total protein on the membrane by $1 \mathrm{x}$ Ponceau $\mathrm{S}$ solution. For more details for buffers and solutions were described in the supplementary materials (S10). Primary antibodies used in this study were displayed as follows: anti-GFP mouse monoclonal B-2 (Santa Cruz), Living Colors ${ }^{\circledR}$ DsRed polyclonal antibody (Clontech Laboratories, Inc) for fluorescent-tagged proteins and antiPGK1 mouse monoclonal 22C5 (Molecular Probes, Invitrogen), anti-Bmh1p rabbit (Michelsen et al., 2006) as loading controls. After blocking the membrane, each primary antibody diluted to 1:1000 was treated for overnight on a shaking incubator at $4{ }^{\circ} \mathrm{C}$ and washed thrice by blocking solution. As secondary antibodies for Odyssey ${ }^{\circledR}$ Sa Infrared Imaging System (LI-COR), IRDye ${ }^{\circledR} 800 \mathrm{CW}$ and 680LT Infrared Dye-conjugated secondary anti-rabbit or anti-mouse antibodies (LI-COR) were used to 1:10,000 dilution in blocking solution for incubation of one hour at room temperature and then washed three times using TBS (0.02\% IGEPAL CA-630). For detection of proteins, membranes were placed on crystal plates and scanned by Odyssey system. Data were quantified and normalized to the control and analyzed by ImageJ software (National Institutes of Health).

\subsubsection{Live cell Imaging}

Live cell imaging for yeast cells were performed by a Delta Vision RT (Applied Precision) microscopy using a 100x/0.35-1.5 Uplan Apo objective with filter sets of the specific band pass of GFP, RFP or TRITC for imaging of living yeast cells, which were taken by a Coolsnap HQ camera (Photometrics) and at least three independent experiments were done under the same conditions in wild type and mutants at room temperature. As for a pulse-chase assay and the colocalization, the samples were grown to $\log$-phase cells to $\mathrm{OD}_{600}$ of between 0.5 and 1.0 after overnight cultures at $30^{\circ} \mathrm{C}$ and, in case of testing endocytosis, the samples were washed twice by fresh media before adding FM $^{\circledR}$ 4-64 (Molecular Probes, Invitrogen), a lipophilic styryl dye for visualizing membranes as a marker of yeast membrane traffic (Vida and Emr, 1995). The samples were then incubated with the dye of a final concentration of $40 \mu \mathrm{M}$ from a stock solution of $16 \mathrm{mM}$ in Dimethyl sulfoxide (DMSO) on ice for 30 minutes as a labeling step and 
spun down gently without washing steps. For imaging, cells were placed back to room temperature for 1 minute and immediately mounted on a microscope slide with a cover slip. Multichannel images of colocalization experiments were analyzed using ImageJ software (National Institutes of Health) and statistical analysis was used by a Student's $t$ test.

\subsubsection{Glycerol Stocks}

Yeast cells overnight inoculated in SC or YPAD media were stocked in glycerol solution at $-80^{\circ} \mathrm{C}$ and liquid nitrogen. For the stock solutions, $400 \mathrm{ul}$ of cultured cells and $400 \mu \mathrm{l}$ of $30 \%$ glycerol were used and mixed together and kept in cryovials for frozen conditions. 


\section{REFERENCES}

Abeliovich, H., Grote, E., Novick, P., and Ferro-Novick, S. (1998). Tlg2p, a yeast syntaxin homolog that resides on the Golgi and endocytic structures. J Biol Chem 273, 11719-11727.

Altschuler, Y., Barbas, S.M., Terlecky, L.J., Tang, K., Hardy, S., Mostov, K.E., and Schmid, S.L. (1998). Redundant and distinct functions for dynamin-1 and dynamin-2 isoforms. The Journal of cell biology 143, 1871-1881.

Altun, Z.F., Herndon, L.A., Crocker, C., Lints, R. and Hall, D.H. (ed.s) (2002-2012). WormAtlas.

Appenzeller-Herzog, C., and Hauri, H.P. (2006). The ER-Golgi intermediate compartment (ERGIC): in search of its identity and function. J Cell Sci 119, 2173-2183.

Ausubel, F.M., Brent, R., Kingston, R.E., Moore, D.D., Seidman, J.G., Smith, J.A., and Struhl, K., eds. (1997). Current Protocols in Molecular Biology (New York: Greene Publishing Associates and Wiley-Interscience).

Barlowe, C., Orci, L., Yeung, T., Hosobuchi, M., Hamamoto, S., Salama, N., Rexach, M.F., Ravazzola, M., Amherdt, M., and Schekman, R. (1994). COPII: a membrane coat formed by Sec proteins that drive vesicle budding from the endoplasmic reticulum. Cell 77, 895-907.

Baugh, L.R., and Hunter, C.P. (2006). MyoD, modularity, and myogenesis: conservation of regulators and redundancy in C. elegans. Genes \& development 20, 3342-3346.

Baumert, M., Maycox, P.R., Navone, F., De Camilli, P., and Jahn, R. (1989). Synaptobrevin: an integral membrane protein of 18,000 daltons present in small synaptic vesicles of rat brain. EMBO J 8, 379-384.

Bertrand, D., Ballivet, M., Gomez, M., Bertrand, S., Phannavong, B., and Gundelfinger, E.D. (1994). Physiological properties of neuronal nicotinic receptors reconstituted from the vertebrate beta 2 subunit and Drosophila alpha subunits. Eur J Neurosci 6, 869-875.

Bicho, C.C., de Lima Alves, F., Chen, Z.A., Rappsilber, J., and Sawin, K.E. (2010). A genetic engineering solution to the "arginine conversion problem" in stable isotope labeling by amino acids in cell culture (SILAC). Molecular \& cellular proteomics : MCP 9, 1567-1577.

Bird, A.F., and Bird, J. (1991). The structure of nematodes, 2nd edn (San Diego: Academic Press).

Boehm, M., and Bonifacino, J.S. (2001). Adaptins: the final recount. Mol Biol Cell 12, 2907-2920.

Boman, A.L., Zhang, C., Zhu, X., and Kahn, R.A. (2000). A family of ADP-ribosylation factor effectors that can alter membrane transport through the trans-Golgi. Mol Biol Cell $11,1241-1255$.

Bonifacino, J.S., and Glick, B.S. (2004). The mechanisms of vesicle budding and fusion. Cell 116, 153-166.

Bonifacino, J.S., and Hierro, A. (2011). Transport according to GARP: receiving retrograde cargo at the trans-Golgi network. Trends Cell Biol 21, 159-167.

Bonifacino, J.S., and Rojas, R. (2006). Retrograde transport from endosomes to the transGolgi network. Nature reviews Molecular cell biology 7, 568-579. 
Boulin, T., Gielen, M., Richmond, J.E., Williams, D.C., Paoletti, P., and Bessereau, J.L. (2008). Eight genes are required for functional reconstitution of the Caenorhabditis elegans levamisole-sensitive acetylcholine receptor. Proc Natl Acad Sci U S A 105, 18590-18595.

Boulin, T., Rapti, G., Briseno-Roa, L., Stigloher, C., Richmond, J.E., Paoletti, P., and Bessereau, J.L. (2012). Positive modulation of a Cys-loop acetylcholine receptor by an auxiliary transmembrane subunit. Nat Neurosci 15, 1374-1381.

Boulon, S., Ahmad, Y., Trinkle-Mulcahy, L., Verheggen, C., Cobley, A., Gregor, P., Bertrand, E., Whitehorn, M., and Lamond, A.I. (2010). Establishment of a protein frequency library and its application in the reliable identification of specific protein interaction partners. Molecular \& cellular proteomics : MCP 9, 861-879.

Bowers, K., Levi, B.P., Patel, F.I., and Stevens, T.H. (2000). The sodium/proton exchanger Nhxlp is required for endosomal protein trafficking in the yeast Saccharomyces cerevisiae. Mol Biol Cell 11, 4277-4294.

Brachmann, C.B., Davies, A., Cost, G.J., Caputo, E., Li, J., Hieter, P., and Boeke, J.D. (1998). Designer deletion strains derived from Saccharomyces cerevisiae S288C: a useful set of strains and plasmids for PCR-mediated gene disruption and other applications. Yeast 14, 115-132.

Braeckman, B.P., Houthoofd, K., and Vanfleteren, J.R. (2009). Intermediary metabolism. WormBook : the online review of C elegans biology, 1-24.

Braun, N.A., Morgan, B., Dick, T.P., and Schwappach, B. (2010). The yeast CLC protein counteracts vesicular acidification during iron starvation. J Cell Sci 123, 2342-2350.

Brenner, S. (1974). The genetics of Caenorhabditis elegans. Genetics 77, 71-94.

Briseno-Roa, L., and Bessereau, J.L. (2014). Proteolytic processing of the extracellular scaffolding protein LEV-9 is required for clustering acetylcholine receptors. J Biol Chem 289, 10967-10974.

Brown, L.A., Jones, A.K., Buckingham, S.D., Mee, C.J., and Sattelle, D.B. (2006). Contributions from Caenorhabditis elegans functional genetics to antiparasitic drug target identification and validation: nicotinic acetylcholine receptors, a case study. Int $\mathrm{J}$ Parasitol 36, 617-624.

Bruneau, E., Sutter, D., Hume, R.I., and Akaaboune, M. (2005). Identification of nicotinic acetylcholine receptor recycling and its role in maintaining receptor density at the neuromuscular junction in vivo. J Neurosci 25, 9949-9959.

Calixto, A., Chelur, D., Topalidou, I., Chen, X., and Chalfie, M. (2010). Enhanced neuronal RNAi in C. elegans using SID-1. Nature methods 7, 554-559.

Camus, G., Jasmin, B.J., and Cartaud, J. (1998). Polarized sorting of nicotinic acetylcholine receptors to the postsynaptic membrane in Torpedo electrocyte. Eur $\mathrm{J}$ Neurosci 10, 839-852.

Carroll, K.S., Hanna, J., Simon, I., Krise, J., Barbero, P., and Pfeffer, S.R. (2001). Role of Rab9 GTPase in facilitating receptor recruitment by TIP47. Science 292, 1373-1376.

Chakravarty, R., Goel, S., and Cai, W. (2014). Nanobody: the "magic bullet" for molecular imaging? Theranostics 4, 386-398.

Chantalat, S., Courbeyrette, R., Senic-Matuglia, F., Jackson, C.L., Goud, B., and Peyroche, A. (2003). A novel Golgi membrane protein is a partner of the ARF exchange factors Gea1p and Gea2p. Mol Biol Cell 14, 2357-2371. 
Chapman-Smith, A., and Cronan, J.E., Jr. (1999). In vivo enzymatic protein biotinylation. Biomolecular engineering 16, 119-125.

Chardin, P., Paris, S., Antonny, B., Robineau, S., Beraud-Dufour, S., Jackson, C.L., and Chabre, M. (1996). A human exchange factor for ARF contains Sec7- and pleckstrinhomology domains. Nature 384, 481-484.

Chavrier, P., Parton, R.G., Hauri, H.P., Simons, K., and Zerial, M. (1990). Localization of low molecular weight GTP binding proteins to exocytic and endocytic compartments. Cell 62, 317-329.

Chen, N., Harris, T.W., Antoshechkin, I., Bastiani, C., Bieri, T., Blasiar, D., Bradnam, K., Canaran, P., Chan, J., Chen, C.K., et al. (2005a). WormBase: a comprehensive data resource for Caenorhabditis biology and genomics. Nucleic Acids Res 33, D383-389.

Chen, S.H., Chen, S., Tokarev, A.A., Liu, F., Jedd, G., and Segev, N. (2005b). Ypt31/32 GTPases and their novel F-box effector protein Rcyl regulate protein recycling. Mol Biol Cell 16, 178-192.

Choi-Rhee, E., Schulman, H., and Cronan, J.E. (2004). Promiscuous protein biotinylation by Escherichia coli biotin protein ligase. Protein science : a publication of the Protein Society 13, 3043-3050.

Christianson, J.C., and Green, W.N. (2004). Regulation of nicotinic receptor expression by the ubiquitin-proteasome system. EMBO J 23, 4156-4165.

Confalonieri, S., Salcini, A.E., Puri, C., Tacchetti, C., and Di Fiore, P.P. (2000). Tyrosine phosphorylation of Eps15 is required for ligand-regulated, but not constitutive, endocytosis. The Journal of cell biology 150, 905-912.

Conibear, E., Cleck, J.N., and Stevens, T.H. (2003). Vps51p mediates the association of the GARP (Vps52/53/54) complex with the late Golgi t-SNARE Tlg1p. Mol Biol Cell 14, 1610-1623.

Conibear, E., and Stevens, T.H. (2000). Vps52p, Vps53p, and Vps54p form a novel multisubunit complex required for protein sorting at the yeast late Golgi. Mol Biol Cell 11, 305-323.

Conner, S.D., and Schmid, S.L. (2003). Regulated portals of entry into the cell. Nature 422, 37-44.

Cosson, P., and Letourneur, F. (1997). Coatomer (COPI)-coated vesicles: role in intracellular transport and protein sorting. Current opinion in cell biology 9, 484-487.

Couve, A., Protopopov, V., and Gerst, J.E. (1995). Yeast synaptobrevin homologs are modified posttranslationally by the addition of palmitate. Proc Natl Acad Sci U S A 92, 5987-5991.

Cowart, R.E., Singleton, F.L., and Hind, J.S. (1993). A comparison of bathophenanthrolinedisulfonic acid and ferrozine as chelators of iron(II) in reduction reactions. Analytical biochemistry 211, 151-155.

Craft, G.E., Chen, A., and Nairn, A.C. (2013). Recent advances in quantitative neuroproteomics. Methods 61, 186-218.

Cunningham, J.T., Rodgers, J.T., Arlow, D.H., Vazquez, F., Mootha, V.K., and Puigserver, P. (2007). mTOR controls mitochondrial oxidative function through a YY1PGC-1alpha transcriptional complex. Nature 450, 736-740.

Cyert, M.S., and Philpott, C.C. (2013). Regulation of cation balance in Saccharomyces cerevisiae. Genetics 193, 677-713. 
Davis-Kaplan, S.R., Askwith, C.C., Bengtzen, A.C., Radisky, D., and Kaplan, J. (1998). Chloride is an allosteric effector of copper assembly for the yeast multicopper oxidase Fet3p: an unexpected role for intracellular chloride channels. Proc Natl Acad Sci U S A 95, 13641-13645.

de Bono, M., and Maricq, A.V. (2005). Neuronal substrates of complex behaviors in C. elegans. Annual review of neuroscience 28, 451-501.

De Meyer, T., Muyldermans, S., and Depicker, A. (2014). Nanobody-based products as research and diagnostic tools. Trends in biotechnology 32, 263-270.

de Renzis, S., Sonnichsen, B., and Zerial, M. (2002). Divalent Rab effectors regulate the sub-compartmental organization and sorting of early endosomes. Nature cell biology 4 , 124-133.

Deeb, S.J., D'Souza, R.C., Cox, J., Schmidt-Supprian, M., and Mann, M. (2012). SuperSILAC allows classification of diffuse large B-cell lymphoma subtypes by their protein expression profiles. Molecular \& cellular proteomics : MCP 11, 77-89.

Dell'Angelica, E.C., Puertollano, R., Mullins, C., Aguilar, R.C., Vargas, J.D., Hartnell, L.M., and Bonifacino, J.S. (2000). GGAs: a family of ADP ribosylation factor-binding proteins related to adaptors and associated with the Golgi complex. The Journal of cell biology 149, 81-94.

Dobreva, I., Fielding, A., Foster, L.J., and Dedhar, S. (2008). Mapping the integrin-linked kinase interactome using SILAC. Journal of proteome research 7, 1740-1749.

Donaldson, J., and Segev, N. (2010). Regulation and coordination of intracellular trafficking. In Trafficking Inside Cells: Pathways, Mechanisms and Regulation, N. Segev, ed. (Springer).

Donaldson, J.G., and Jackson, C.L. (2000). Regulators and effectors of the ARF GTPases. Current opinion in cell biology 12, 475-482.

Donaldson, J.G., and Lippincott-Schwartz, J. (2000). Sorting and signaling at the Golgi complex. Cell 101, 693-696.

Eddy, J.A., Hood, L., Price, N.D., and Geman, D. (2010). Identifying tightly regulated and variably expressed networks by Differential Rank Conservation (DIRAC). PLoS computational biology 6, e1000792.

Eimer, S., Gottschalk, A., Hengartner, M., Horvitz, H.R., Richmond, J., Schafer, W.R., and Bessereau, J.L. (2007). Regulation of nicotinic receptor trafficking by the transmembrane Golgi protein UNC-50. EMBO J 26, 4313-4323.

Ellgaard, L., Molinari, M., and Helenius, A. (1999). Setting the standards: quality control in the secretory pathway. Science $286,1882-1888$.

Emmott, E., and Goodfellow, I. (2014). Identification of Protein Interaction Partners in Mammalian Cells Using SILAC-immunoprecipitation Quantitative Proteomics. Journal of visualized experiments : JoVE.

Emmott, E., Munday, D., Bickerton, E., Britton, P., Rodgers, M.A., Whitehouse, A., Zhou, E.M., and Hiscox, J.A. (2013). The cellular interactome of the coronavirus infectious bronchitis virus nucleocapsid protein and functional implications for virus biology. Journal of virology 87, 9486-9500.

Eugster, A., Frigerio, G., Dale, M., and Duden, R. (2000). COP I domains required for coatomer integrity, and novel interactions with ARF and ARF-GAP. EMBO J 19, 39053917. 
Fares, H., and Greenwald, I. (2001). Genetic analysis of endocytosis in Caenorhabditis elegans: coelomocyte uptake defective mutants. Genetics 159, 133-145.

Fath, S., Mancias, J.D., Bi, X., and Goldberg, J. (2007). Structure and organization of coat proteins in the COPII cage. Cell 129, 1325-1336.

Findlay, G.D., Yi, X., Maccoss, M.J., and Swanson, W.J. (2008). Proteomics reveals novel Drosophila seminal fluid proteins transferred at mating. PLoS biology 6, e178.

Fire, A., Xu, S., Montgomery, M.K., Kostas, S.A., Driver, S.E., and Mello, C.C. (1998). Potent and specific genetic interference by double-stranded RNA in Caenorhabditis elegans. Nature 391, 806-811.

Fitzgerald, J., Kennedy, D., Viseshakul, N., Cohen, B.N., Mattick, J., Bateman, J.F., and Forsayeth, J.R. (2000). UNCL, the mammalian homologue of UNC-50, is an inner nuclear membrane RNA-binding protein. Brain Res 877, 110-123.

Fleming, J.T., Squire, M.D., Barnes, T.M., Tornoe, C., Matsuda, K., Ahnn, J., Fire, A., Sulston, J.E., Barnard, E.A., Sattelle, D.B., et al. (1997). Caenorhabditis elegans levamisole resistance genes lev-1, unc-29, and unc-38 encode functional nicotinic acetylcholine receptor subunits. J Neurosci 17, 5843-5857.

Forsayeth, J.R., Gu, Y., and Hall, Z.W. (1992). BiP forms stable complexes with unassembled subunits of the acetylcholine receptor in transfected COS cells and in C2 muscle cells. The Journal of cell biology 117, 841-847.

Fotin, A., Cheng, Y., Sliz, P., Grigorieff, N., Harrison, S.C., Kirchhausen, T., and Walz, T. (2004). Molecular model for a complete clathrin lattice from electron cryomicroscopy. Nature 432, 573-579.

Fredens, J., Engholm-Keller, K., Giessing, A., Pultz, D., Larsen, M.R., Hojrup, P., Moller-Jensen, J., and Faergeman, N.J. (2011). Quantitative proteomics by amino acid labeling in C. elegans. Nature methods $8,845-847$.

Friedlander, R., Jarosch, E., Urban, J., Volkwein, C., and Sommer, T. (2000). A regulatory link between ER-associated protein degradation and the unfolded-protein response. Nature cell biology 2, 379-384.

Gabriely, G., Kama, R., and Gerst, J.E. (2007). Involvement of specific COPI subunits in protein sorting from the late endosome to the vacuole in yeast. Mol Cell Biol 27, 526540 .

Galan, J.M., Wiederkehr, A., Seol, J.H., Haguenauer-Tsapis, R., Deshaies, R.J., Riezman, H., and Peter, M. (2001). Skplp and the F-box protein Rcylp form a non-SCF complex involved in recycling of the SNARE Snclp in yeast. Mol Cell Biol 21, 3105-3117.

Gallagher, M.J., Ding, L., Maheshwari, A., and Macdonald, R.L. (2007). The GABAA receptor alphal subunit epilepsy mutation A322D inhibits transmembrane helix formation and causes proteasomal degradation. Proc Natl Acad Sci U S A 104, 1299913004.

Gally, C., Eimer, S., Richmond, J.E., and Bessereau, J.L. (2004). A transmembrane protein required for acetylcholine receptor clustering in Caenorhabditis elegans. Nature 431, 578-582.

Garcia, L.R., Mehta, P., and Sternberg, P.W. (2001). Regulation of distinct muscle behaviors controls the C. elegans male's copulatory spicules during mating. Cell 107, 777-788. 
Gaxiola, R.A., Rao, R., Sherman, A., Grisafi, P., Alper, S.L., and Fink, G.R. (1999). The Arabidopsis thaliana proton transporters, AtNhx1 and Avp1, can function in cation detoxification in yeast. Proc Natl Acad Sci U S A 96, 1480-1485.

Gaxiola, R.A., Yuan, D.S., Klausner, R.D., and Fink, G.R. (1998). The yeast CLC chloride channel functions in cation homeostasis. Proc Natl Acad Sci U S A 95, 40464050.

Geiger, T., Cox, J., Ostasiewicz, P., Wisniewski, J.R., and Mann, M. (2010). SuperSILAC mix for quantitative proteomics of human tumor tissue. Nature methods 7, 383385.

Geiger, T., Wisniewski, J.R., Cox, J., Zanivan, S., Kruger, M., Ishihama, Y., and Mann, M. (2011). Use of stable isotope labeling by amino acids in cell culture as a spike-in standard in quantitative proteomics. Nature protocols 6, 147-157.

Gelman, M.S., Chang, W., Thomas, D.Y., Bergeron, J.J., and Prives, J.M. (1995). Role of the endoplasmic reticulum chaperone calnexin in subunit folding and assembly of nicotinic acetylcholine receptors. J Biol Chem 270, 15085-15092.

Gendrel, M., Rapti, G., Richmond, J.E., and Bessereau, J.L. (2009). A secreted complement-control-related protein ensures acetylcholine receptor clustering. Nature 461, 992-996.

Gerst, J.E., Rodgers, L., Riggs, M., and Wigler, M. (1992). SNC1, a yeast homolog of the synaptic vesicle-associated membrane protein/synaptobrevin gene family: genetic interactions with the RAS and CAP genes. Proc Natl Acad Sci U S A 89, 4338-4342.

Ghosh, P., Dahms, N.M., and Kornfeld, S. (2003). Mannose 6-phosphate receptors: new twists in the tale. Nature reviews Molecular cell biology 4, 202-212.

Gietz, D., St Jean, A., Woods, R.A., and Schiestl, R.H. (1992). Improved method for high efficiency transformation of intact yeast cells. Nucleic Acids Res 20, 1425.

Gillingham, A.K., and Munro, S. (2007). The small G proteins of the Arf family and their regulators. Annual review of cell and developmental biology 23, 579-611.

Gillooly, D.J., Morrow, I.C., Lindsay, M., Gould, R., Bryant, N.J., Gaullier, J.M., Parton, R.G., and Stenmark, H. (2000). Localization of phosphatidylinositol 3-phosphate in yeast and mammalian cells. EMBO J 19, 4577-4588.

Girard, L.R., Fiedler, T.J., Harris, T.W., Carvalho, F., Antoshechkin, I., Han, M., Sternberg, P.W., Stein, L.D., and Chalfie, M. (2007). WormBook: the online review of Caenorhabditis elegans biology. Nucleic Acids Res 35, D472-475.

Goldberg, J. (1998). Structural basis for activation of ARF GTPase: mechanisms of guanine nucleotide exchange and GTP-myristoyl switching. Cell 95, 237-248.

Goldstein, A.L., and McCusker, J.H. (1999). Three new dominant drug resistance cassettes for gene disruption in Saccharomyces cerevisiae. Yeast 15, 1541-1553.

Gotti, C., and Clementi, F. (2004). Neuronal nicotinic receptors: from structure to pathology. Prog Neurobiol 74, 363-396.

Gould, G.W., and Lippincott-Schwartz, J. (2009). New roles for endosomes: from vesicular carriers to multi-purpose platforms. Nature reviews Molecular cell biology 10, 287-292.

Gouw, J.W., Krijgsveld, J., and Heck, A.J. (2010). Quantitative proteomics by metabolic labeling of model organisms. Molecular \& cellular proteomics : MCP 9, 11-24.

Gouw, J.W., Tops, B.B., and Krijgsveld, J. (2011). Metabolic labeling of model organisms using heavy nitrogen (15N). Methods Mol Biol 753, 29-42. 
Grant, B., and Hirsh, D. (1999). Receptor-mediated endocytosis in the Caenorhabditis elegans oocyte. Mol Biol Cell 10, 4311-4326.

Grant, B.D., and Donaldson, J.G. (2009). Pathways and mechanisms of endocytic recycling. Nature reviews Molecular cell biology 10, 597-608.

Grant, B.D., and Sato, M. (2006). Intracellular trafficking. WormBook : the online review of C elegans biology, 1-9.

Green, W.N., and Millar, N.S. (1995). Ion-channel assembly. Trends in neurosciences 18, 280-287.

Greene, J.R., Brown, N.H., DiDomenico, B.J., Kaplan, J., and Eide, D.J. (1993). The GEF1 gene of Saccharomyces cerevisiae encodes an integral membrane protein; mutations in which have effects on respiration and iron-limited growth. Molecular \& general genetics : MGG 241, 542-553.

Groot, A.J., Verheesen, P., Westerlaken, E.J., Gort, E.H., van der Groep, P., Bovenschen, N., van der Wall, E., van Diest, P.J., and Shvarts, A. (2006). Identification by phage display of single-domain antibody fragments specific for the ODD domain in hypoxiainducible factor 1alpha. Laboratory investigation; a journal of technical methods and pathology $86,345-356$.

Gruhler, A., Schulze, W.X., Matthiesen, R., Mann, M., and Jensen, O.N. (2005). Stable isotope labeling of Arabidopsis thaliana cells and quantitative proteomics by mass spectrometry. Molecular \& cellular proteomics : MCP 4, 1697-1709.

Gu, F., Crump, C.M., and Thomas, G. (2001). Trans-Golgi network sorting. Cell Mol Life Sci 58, 1067-1084.

Gurunathan, S., Chapman-Shimshoni, D., Trajkovic, S., and Gerst, J.E. (2000). Yeast exocytic v-SNAREs confer endocytosis. Mol Biol Cell 11, 3629-3643.

Habibi-Babadi, N., Su, A., de Carvalho, C.E., and Colavita, A. (2010). The N-glycanase png-1 acts to limit axon branching during organ formation in Caenorhabditis elegans. $\mathrm{J}$ Neurosci 30, 1766-1776.

Halevi, S., McKay, J., Palfreyman, M., Yassin, L., Eshel, M., Jorgensen, E., and Treinin, M. (2002). The C. elegans ric-3 gene is required for maturation of nicotinic acetylcholine receptors. EMBO J 21, 1012-1020.

Halevi, S., Yassin, L., Eshel, M., Sala, F., Sala, S., Criado, M., and Treinin, M. (2003). Conservation within the RIC-3 gene family. Effectors of mammalian nicotinic acetylcholine receptor expression. J Biol Chem 278, 34411-34417.

Hamers-Casterman, C., Atarhouch, T., Muyldermans, S., Robinson, G., Hamers, C., Songa, E.B., Bendahman, N., and Hamers, R. (1993). Naturally occurring antibodies devoid of light chains. Nature 363, 446-448.

Hauri, H.P., Kappeler, F., Andersson, H., and Appenzeller, C. (2000). ERGIC-53 and traffic in the secretory pathway. J Cell Sci 113 ( Pt 4), 587-596.

Hayes, G.L., Brown, F.C., Haas, A.K., Nottingham, R.M., Barr, F.A., and Pfeffer, S.R. (2009). Multiple Rab GTPase binding sites in GCC185 suggest a model for vesicle tethering at the trans-Golgi. Mol Biol Cell 20, 209-217.

Helsens, K., Martens, L., Vandekerckhove, J., and Gevaert, K. (2011). Mass spectrometry-driven proteomics: an introduction. Methods Mol Biol 753, 1-27.

Hettema, E.H., Lewis, M.J., Black, M.W., and Pelham, H.R. (2003). Retromer and the sorting nexins Snx4/41/42 mediate distinct retrieval pathways from yeast endosomes. EMBO J 22, 548-557. 
Hobert, O., Mori, I., Yamashita, Y., Honda, H., Ohshima, Y., Liu, Y., and Ruvkun, G. (1997). Regulation of interneuron function in the C. elegans thermoregulatory pathway by the ttx-3 LIM homeobox gene. Neuron 19, 345-357.

Hoffman, G.R., Rahl, P.B., Collins, R.N., and Cerione, R.A. (2003). Conserved structural motifs in intracellular trafficking pathways: structure of the gammaCOP appendage domain. Molecular cell 12, 615-625.

Huang, K.M., D'Hondt, K., Riezman, H., and Lemmon, S.K. (1999). Clathrin functions in the absence of heterotetrameric adaptors and AP180-related proteins in yeast. EMBO J 18, 3897-3908.

Huh, W.K., Falvo, J.V., Gerke, L.C., Carroll, A.S., Howson, R.W., Weissman, J.S., and O'Shea, E.K. (2003). Global analysis of protein localization in budding yeast. Nature 425, 686-691.

Hulme, S.E., and Whitesides, G.M. (2011). Chemistry and the worm: Caenorhabditis elegans as a platform for integrating chemical and biological research. Angew Chem Int Ed Engl 50, 4774-4807.

Husson, S.J., Mertens, I., Janssen, T., Lindemans, M., and Schoofs, L. (2007). Neuropeptidergic signaling in the nematode Caenorhabditis elegans. Prog Neurobiol 82, 33-55.

Ihara, M., Matsuda, K., Otake, M., Kuwamura, M., Shimomura, M., Komai, K., Akamatsu, M., Raymond, V., and Sattelle, D.B. (2003). Diverse actions of neonicotinoids on chicken alpha7, alpha4beta2 and Drosophila-chicken SADbeta2 and ALSbeta2 hybrid nicotinic acetylcholine receptors expressed in Xenopus laevis oocytes. Neuropharmacology 45, 133-144.

Ihara, S., and Nishiwaki, K. (2007). Prodomain-dependent tissue targeting of an ADAMTS protease controls cell migration in Caenorhabditis elegans. EMBO J 26, 26072620.

Ishizaki, R., Shin, H.W., Mitsuhashi, H., and Nakayama, K. (2008). Redundant roles of BIG2 and BIG1, guanine-nucleotide exchange factors for ADP-ribosylation factors in membrane traffic between the trans-Golgi network and endosomes. Mol Biol Cell 19, 2650-2660.

Jackson, C.L., and Casanova, J.E. (2000). Turning on ARF: the Sec7 family of guaninenucleotide-exchange factors. Trends Cell Biol 10, 60-67.

Jahn, R., and Scheller, R.H. (2006). SNAREs--engines for membrane fusion. Nature reviews Molecular cell biology 7, 631-643.

Jarosch, E., Lenk, U., and Sommer, T. (2003). Endoplasmic reticulum-associated protein degradation. International review of cytology 223, 39-81.

Jobling, S.A., Jarman, C., Teh, M.M., Holmberg, N., Blake, C., and Verhoeyen, M.E. (2003). Immunomodulation of enzyme function in plants by single-domain antibody fragments. Nature biotechnology 21, 77-80.

Kalatzis, V., Cherqui, S., Antignac, C., and Gasnier, B. (2001). Cystinosin, the protein defective in cystinosis, is a $\mathrm{H}(+)$-driven lysosomal cystine transporter. EMBO J 20, 59405949.

Kaletta, T., and Hengartner, M.O. (2006). Finding function in novel targets: C. elegans as a model organism. Nature reviews Drug discovery 5, 387-398. 
Kamath, R.S., Fraser, A.G., Dong, Y., Poulin, G., Durbin, R., Gotta, M., Kanapin, A., Le Bot, N., Moreno, S., Sohrmann, M., et al. (2003). Systematic functional analysis of the Caenorhabditis elegans genome using RNAi. Nature 421, 231-237.

Kamath, R.S., Martinez-Campos, M., Zipperlen, P., Fraser, A.G., and Ahringer, J. (2001). Effectiveness of specific RNA-mediated interference through ingested double-stranded RNA in Caenorhabditis elegans. Genome biology 2, RESEARCH0002.

Keller, S.H., Lindstrom, J., Ellisman, M., and Taylor, P. (2001). Adjacent basic amino acid residues recognized by the COP I complex and ubiquitination govern endoplasmic reticulum to cell surface trafficking of the nicotinic acetylcholine receptor alpha-Subunit. J Biol Chem 276, 18384-18391.

Kim, H.J., Choi, Y.S., Jeong, M.J., Kim, B.O., Lim, S.H., Kim do, K., Kim, C.K., and Park, J.C. (2007). Expression of UNCL during development of periodontal tissue and response of periodontal ligament fibroblasts to mechanical stress in vivo and in vitro. Cell and tissue research 327, 25-31.

Kim, J., Poole, D.S., Waggoner, L.E., Kempf, A., Ramirez, D.S., Treschow, P.A., and Schafer, W.R. (2001). Genes affecting the activity of nicotinic receptors involved in Caenorhabditis elegans egg-laying behavior. Genetics 157, 1599-1610.

Kirchhausen, T. (2000). Three ways to make a vesicle. Nature reviews Molecular cell biology 1, 187-198.

Kirchhofer, A., Helma, J., Schmidthals, K., Frauer, C., Cui, S., Karcher, A., Pellis, M., Muyldermans, S., Casas-Delucchi, C.S., Cardoso, M.C., et al. (2010). Modulation of protein properties in living cells using nanobodies. Nature structural \& molecular biology 17, 133-138.

Knop, M., Barr, F., Riedel, C.G., Heckel, T., and Reichel, C. (2002). Improved version of the red fluorescent protein (drFP583/DsRed/RFP). BioTechniques 33, 592, 594, 596-598 passim.

Krijgsveld, J., Ketting, R.F., Mahmoudi, T., Johansen, J., Artal-Sanz, M., Verrijzer, C.P., Plasterk, R.H., and Heck, A.J. (2003). Metabolic labeling of C. elegans and D. melanogaster for quantitative proteomics. Nature biotechnology 21, 927-931.

Kruger, M., Moser, M., Ussar, S., Thievessen, I., Luber, C.A., Forner, F., Schmidt, S., Zanivan, S., Fassler, R., and Mann, M. (2008). SILAC mouse for quantitative proteomics uncovers kindlin-3 as an essential factor for red blood cell function. Cell 134, 353-364.

Kubala, M.H., Kovtun, O., Alexandrov, K., and Collins, B.M. (2010). Structural and thermodynamic analysis of the GFP:GFP-nanobody complex. Protein science : a publication of the Protein Society 19, 2389-2401.

Kwon, K., and Beckett, D. (2000). Function of a conserved sequence motif in biotin holoenzyme synthetases. Protein science : a publication of the Protein Society 9, 15301539.

Lafourcade, C., Galan, J.M., Gloor, Y., Haguenauer-Tsapis, R., and Peter, M. (2004). The GTPase-activating enzyme Gyp1p is required for recycling of internalized membrane material by inactivation of the Rab/Ypt GTPase Ypt1p. Mol Cell Biol 24, 3815-3826.

Lahm, H.W., and Langen, H. (2000). Mass spectrometry: a tool for the identification of proteins separated by gels. Electrophoresis 21, 2105-2114.

Lang, T., and Jahn, R. (2008). Core proteins of the secretory machinery. Handbook of experimental pharmacology, 107-127. 
Larance, M., Bailly, A.P., Pourkarimi, E., Hay, R.T., Buchanan, G., Coulthurst, S., Xirodimas, D.P., Gartner, A., and Lamond, A.I. (2011). Stable-isotope labeling with amino acids in nematodes. Nature methods $8,849-851$.

Le Novere, N., Corringer, P.J., and Changeux, J.P. (2002). The diversity of subunit composition in nAChRs: evolutionary origins, physiologic and pharmacologic consequences. Journal of neurobiology 53, 447-456.

Lee, C., and Goldberg, J. (2010). Structure of coatomer cage proteins and the relationship among COPI, COPII, and clathrin vesicle coats. Cell 142, 123-132.

Lee, M.C., Miller, E.A., Goldberg, J., Orci, L., and Schekman, R. (2004). Bi-directional protein transport between the ER and Golgi. Annual review of cell and developmental biology 20, 87-123.

Letourneur, F., Gaynor, E.C., Hennecke, S., Demolliere, C., Duden, R., Emr, S.D., Riezman, H., and Cosson, P. (1994). Coatomer is essential for retrieval of dilysine-tagged proteins to the endoplasmic reticulum. Cell 79, 1199-1207.

Lewis, J.A., Wu, C.H., Berg, H., and Levine, J.H. (1980). The genetics of levamisole resistance in the nematode Caenorhabditis elegans. Genetics 95, 905-928.

Lewis, M.J., Nichols, B.J., Prescianotto-Baschong, C., Riezman, H., and Pelham, H.R. (2000). Specific retrieval of the exocytic SNARE Snc1p from early yeast endosomes. Mol Biol Cell 11, 23-38.

Li, H., Li, H.F., Felder, R.A., Periasamy, A., and Jose, P.A. (2008). Rab4 and Rab11 coordinately regulate the recycling of angiotensin II type I receptor as demonstrated by fluorescence resonance energy transfer microscopy. Journal of biomedical optics 13, 031206.

Li, S., Armstrong, C.M., Bertin, N., Ge, H., Milstein, S., Boxem, M., Vidalain, P.O., Han, J.D., Chesneau, A., Hao, T., et al. (2004). A map of the interactome network of the metazoan C. elegans. Science 303, 540-543.

Lode, A., Paret, C., and Rodel, G. (2002). Molecular characterization of Saccharomyces cerevisiae Sco2p reveals a high degree of redundancy with Sco1p. Yeast 19, 909-922.

Lowery, J., Szul, T., Styers, M., Holloway, Z., Oorschot, V., Klumperman, J., and Sztul, E. (2013). The Sec7 guanine nucleotide exchange factor GBF1 regulates membrane recruitment of BIG1 and BIG2 guanine nucleotide exchange factors to the trans-Golgi network (TGN). J Biol Chem 288, 11532-11545.

Luo, L., Hannemann, M., Koenig, S., Hegermann, J., Ailion, M., Cho, M.K., Sasidharan, N., Zweckstetter, M., Rensing, S.A., and Eimer, S. (2011). The Caenorhabditis elegans GARP complex contains the conserved Vps51 subunit and is required to maintain lysosomal morphology. Mol Biol Cell 22, 2564-2578.

Mahoney, T.R., Luo, S., and Nonet, M.L. (2006). Analysis of synaptic transmission in Caenorhabditis elegans using an aldicarb-sensitivity assay. Nature protocols 1, 17721777.

Mallard, F., Antony, C., Tenza, D., Salamero, J., Goud, B., and Johannes, L. (1998). Direct pathway from early/recycling endosomes to the Golgi apparatus revealed through the study of shiga toxin B-fragment transport. The Journal of cell biology 143, 973-990.

Mallard, F., Tang, B.L., Galli, T., Tenza, D., Saint-Pol, A., Yue, X., Antony, C., Hong, W., Goud, B., and Johannes, L. (2002). Early/recycling endosomes-to-TGN transport involves two SNARE complexes and a Rab6 isoform. The Journal of cell biology 156, 653-664. 
Mallet, W.G., and Maxfield, F.R. (1999). Chimeric forms of furin and TGN38 are transported with the plasma membrane in the trans-Golgi network via distinct endosomal pathways. The Journal of cell biology 146, 345-359.

Mann, M. (2006). Functional and quantitative proteomics using SILAC. Nature reviews Molecular cell biology 7, 952-958.

Matanis, T., Akhmanova, A., Wulf, P., Del Nery, E., Weide, T., Stepanova, T., Galjart, N., Grosveld, F., Goud, B., De Zeeuw, C.I., et al. (2002). Bicaudal-D regulates COPIindependent Golgi-ER transport by recruiting the dynein-dynactin motor complex. Nature cell biology 4, 986-992.

Mayor, S., and Pagano, R.E. (2007). Pathways of clathrin-independent endocytosis. Nature reviews Molecular cell biology 8, 603-612.

McEwen, J.M., and Kaplan, J.M. (2008). UNC-18 promotes both the anterograde trafficking and synaptic function of syntaxin. Mol Biol Cell 19, 3836-3846.

McKay, J.P., Raizen, D.M., Gottschalk, A., Schafer, W.R., and Avery, L. (2004). eat-2 and eat-18 are required for nicotinic neurotransmission in the Caenorhabditis elegans pharynx. Genetics 166, 161-169.

McMahon, H.T., and Mills, I.G. (2004). COP and clathrin-coated vesicle budding: different pathways, common approaches. Current opinion in cell biology 16, 379-391.

Mello, C., and Fire, A. (1995). DNA transformation. Methods in cell biology 48, 451482.

Mello, C.C., Kramer, J.M., Stinchcomb, D., and Ambros, V. (1991). Efficient gene transfer in C.elegans: extrachromosomal maintenance and integration of transforming sequences. EMBO J 10, 3959-3970.

Merlie, J.P., and Lindstrom, J. (1983). Assembly in vivo of mouse muscle acetylcholine receptor: identification of an alpha subunit species that may be an assembly intermediate. Cell 34, 747-757.

Merrifield, C.J., Perrais, D., and Zenisek, D. (2005). Coupling between clathrin-coatedpit invagination, cortactin recruitment, and membrane scission observed in live cells. Cell 121, 593-606.

Meyer, S., Savaresi, S., Forster, I.C., and Dutzler, R. (2007). Nucleotide recognition by the cytoplasmic domain of the human chloride transporter $\mathrm{ClC}-5$. Nature structural \& molecular biology 14, 60-67.

Michelsen, K., Mrowiec, T., Duderstadt, K.E., Frey, S., Minor, D.L., Mayer, M.P., and Schwappach, B. (2006). A multimeric membrane protein reveals 14-3-3 isoform specificity in forward transport in yeast. Traffic 7, 903-916.

Mittler, G., Butter, F., and Mann, M. (2009). A SILAC-based DNA protein interaction screen that identifies candidate binding proteins to functional DNA elements. Genome research 19, 284-293.

Mouratou, B., Biou, V., Joubert, A., Cohen, J., Shields, D.J., Geldner, N., Jurgens, G., Melancon, P., and Cherfils, J. (2005). The domain architecture of large guanine nucleotide exchange factors for the small GTP-binding protein Arf. BMC genomics 6, 20.

Mumberg, D., Muller, R., and Funk, M. (1994). Regulatable promoters of Saccharomyces cerevisiae: comparison of transcriptional activity and their use for heterologous expression. Nucleic Acids Res 22, 5767-5768. 
Murphy, C.T., McCarroll, S.A., Bargmann, C.I., Fraser, A., Kamath, R.S., Ahringer, J., Li, H., and Kenyon, C. (2003). Genes that act downstream of DAF-16 to influence the lifespan of Caenorhabditis elegans. Nature 424, 277-283.

Muyldermans, S., Baral, T.N., Retamozzo, V.C., De Baetselier, P., De Genst, E., Kinne, J., Leonhardt, H., Magez, S., Nguyen, V.K., Revets, H., et al. (2009). Camelid immunoglobulins and nanobody technology. Veterinary immunology and immunopathology 128, 178-183.

Nabeel-Shah, S., Ashraf, K., Pearlman, R.E., and Fillingham, J. (2014). Molecular evolution of NASP and conserved histone H3/H4 transport pathway. BMC evolutionary biology 14, 139.

Naor, D., Nedvetzki, S., Assayag, N., Thurmond, R.L., Huang, J.F., and Turley, E.A. (2005). The mechanism of molecular redundancy in autoimmune inflammation in the context of CD44 deficiency. Annals of the New York Academy of Sciences 1050, 52-63.

Ni, X., and Morales, C.R. (2006). The lysosomal trafficking of acid sphingomyelinase is mediated by sortilin and mannose 6-phosphate receptor. Traffic 7, 889-902.

Nichols, B.J., Holthuis, J.C., and Pelham, H.R. (1998). The Sec1p homologue Vps45p binds to the syntaxin Tlg2p. European journal of cell biology 77, 263-268.

Nielsen, M.S., Madsen, P., Christensen, E.I., Nykjaer, A., Gliemann, J., Kasper, D., Pohlmann, R., and Petersen, C.M. (2001). The sortilin cytoplasmic tail conveys Golgiendosome transport and binds the VHS domain of the GGA2 sorting protein. EMBO J 20, 2180-2190.

Nishimoto-Morita, K., Shin, H.W., Mitsuhashi, H., Kitamura, M., Zhang, Q., Johannes, L., and Nakayama, K. (2009). Differential effects of depletion of ARL1 and ARFRP1 on membrane trafficking between the trans-Golgi network and endosomes. J Biol Chem 284, 10583-10592.

Oda, Y., Huang, K., Cross, F.R., Cowburn, D., and Chait, B.T. (1999). Accurate quantitation of protein expression and site-specific phosphorylation. Proc Natl Acad Sci U S A 96, 6591-6596.

Ong, S.E., Blagoev, B., Kratchmarova, I., Kristensen, D.B., Steen, H., Pandey, A., and Mann, M. (2002). Stable isotope labeling by amino acids in cell culture, SILAC, as a simple and accurate approach to expression proteomics. Molecular \& cellular proteomics : MCP 1, 376-386.

Ong, S.E., and Mann, M. (2006). A practical recipe for stable isotope labeling by amino acids in cell culture (SILAC). Nature protocols 1, 2650-2660.

Panic, B., Whyte, J.R., and Munro, S. (2003). The ARF-like GTPases Arl1p and Arl3p act in a pathway that interacts with vesicle-tethering factors at the Golgi apparatus. Current biology : CB 13, 405-410.

Payne, G.C.a.G.S. (2009). Trafficking Inside Cells: Pathways, Mechanisms and Regulation_Overview of Protein Trafficking Mechanisms. Landes Bioscience and Springer Science+Business Media.

Pinan-Lucarre, B., Tu, H., Pierron, M., Cruceyra, P.I., Zhan, H., Stigloher, C., Richmond, J.E., and Bessereau, J.L. (2014). C. elegans Punctin specifies cholinergic versus GABAergic identity of postsynaptic domains. Nature.

Protopopov, V., Govindan, B., Novick, P., and Gerst, J.E. (1993). Homologs of the synaptobrevin/VAMP family of synaptic vesicle proteins function on the late secretory pathway in S. cerevisiae. Cell 74, 855-861. 
Puertollano, R., Aguilar, R.C., Gorshkova, I., Crouch, R.J., and Bonifacino, J.S. (2001a). Sorting of mannose 6-phosphate receptors mediated by the GGAs. Science 292, 17121716.

Puertollano, R., Randazzo, P.A., Presley, J.F., Hartnell, L.M., and Bonifacino, J.S. (2001b). The GGAs promote ARF-dependent recruitment of clathrin to the TGN. Cell 105, 93-102.

Quenneville, N.R., Chao, T.Y., McCaffery, J.M., and Conibear, E. (2006). Domains within the GARP subunit Vps54 confer separate functions in complex assembly and early endosome recognition. Mol Biol Cell 17, 1859-1870.

Rapti, G., Richmond, J., and Bessereau, J.L. (2011). A single immunoglobulin-domain protein required for clustering acetylcholine receptors in C. elegans. EMBO J 30, 706718.

Ren, X.Q., Cheng, S.B., Treuil, M.W., Mukherjee, J., Rao, J., Braunewell, K.H., Lindstrom, J.M., and Anand, R. (2005). Structural determinants of alpha4beta2 nicotinic acetylcholine receptor trafficking. J Neurosci 25, 6676-6686.

Reynolds, E.S. (1963). The use of lead citrate at high $\mathrm{pH}$ as an electron-opaque stain in electron microscopy. The Journal of cell biology 17, 208-212.

Rhee, H.W., Zou, P., Udeshi, N.D., Martell, J.D., Mootha, V.K., Carr, S.A., and Ting, A.Y. (2013). Proteomic mapping of mitochondria in living cells via spatially restricted enzymatic tagging. Science 339, 1328-1331.

Richard, M., Boulin, T., Robert, V.J., Richmond, J.E., and Bessereau, J.L. (2013). Biosynthesis of ionotropic acetylcholine receptors requires the evolutionarily conserved ER membrane complex. Proc Natl Acad Sci U S A 110, E1055-1063.

Richardson, B.C., McDonold, C.M., and Fromme, J.C. (2012). The Sec7 Arf-GEF is recruited to the trans-Golgi network by positive feedback. Developmental cell 22, 799810.

Richmond, J.E., and Jorgensen, E.M. (1999). One GABA and two acetylcholine receptors function at the C. elegans neuromuscular junction. Nat Neurosci 2, 791-797.

Rink, J., Ghigo, E., Kalaidzidis, Y., and Zerial, M. (2005). Rab conversion as a mechanism of progression from early to late endosomes. Cell 122, 735-749.

Robinson, M., Poon, P.P., Schindler, C., Murray, L.E., Kama, R., Gabriely, G., Singer, R.A., Spang, A., Johnston, G.C., and Gerst, J.E. (2006). The Ges1 Arf-GAP mediates Snc1,2 v-SNARE retrieval to the Golgi in yeast. Mol Biol Cell 17, 1845-1858.

Robinson, M.S. (2004). Adaptable adaptors for coated vesicles. Trends Cell Biol 14, 167174.

Rohn, W.M., Rouille, Y., Waguri, S., and Hoflack, B. (2000). Bi-directional trafficking between the trans-Golgi network and the endosomal/lysosomal system. J Cell Sci 113 ( Pt 12), 2093-2101.

Rojas, R., van Vlijmen, T., Mardones, G.A., Prabhu, Y., Rojas, A.L., Mohammed, S., Heck, A.J., Raposo, G., van der Sluijs, P., and Bonifacino, J.S. (2008). Regulation of retromer recruitment to endosomes by sequential action of Rab5 and Rab7. The Journal of cell biology 183, 513-526.

Rostaing, P., Weimer, R.M., Jorgensen, E.M., Triller, A., and Bessereau, J.L. (2004). Preservation of immunoreactivity and fine structure of adult $\mathrm{C}$. elegans tissues using high-pressure freezing. J Histochem Cytochem 52, 1-12. 
Rothbauer, U., Zolghadr, K., Muyldermans, S., Schepers, A., Cardoso, M.C., and Leonhardt, H. (2008). A versatile nanotrap for biochemical and functional studies with fluorescent fusion proteins. Molecular \& cellular proteomics : MCP 7, 282-289.

Rothbauer, U., Zolghadr, K., Tillib, S., Nowak, D., Schermelleh, L., Gahl, A., Backmann, N., Conrath, K., Muyldermans, S., Cardoso, M.C., et al. (2006). Targeting and tracing antigens in live cells with fluorescent nanobodies. Nature methods 3, 887-889.

Rothman, J.E., and Warren, G. (1994). Implications of the SNARE hypothesis for intracellular membrane topology and dynamics. Current biology: CB 4, 220-233.

Rouille, Y., Rohn, W., and Hoflack, B. (2000). Targeting of lysosomal proteins. Seminars in cell \& developmental biology 11, 165-171.

Roux, K.J., Kim, D.I., and Burke, B. (2013). BioID: a screen for protein-protein interactions. Current protocols in protein science / editorial board, John E Coligan [et al] 74, Unit 1923.

Roux, K.J., Kim, D.I., Raida, M., and Burke, B. (2012). A promiscuous biotin ligase fusion protein identifies proximal and interacting proteins in mammalian cells. The Journal of cell biology 196, 801-810.

Sambrook, J.J., and Russell, D.W. (2001). Molecular cloning: A Laboratory Manual.

Scheel, O., Zdebik, A.A., Lourdel, S., and Jentsch, T.J. (2005). Voltage-dependent electrogenic chloride/proton exchange by endosomal CLC proteins. Nature 436, 424-427. Schekman, R., and Orci, L. (1996). Coat proteins and vesicle budding. Science 271, 1526-1533.

Schlager, M.A., Kapitein, L.C., Grigoriev, I., Burzynski, G.M., Wulf, P.S., Keijzer, N., de Graaff, E., Fukuda, M., Shepherd, I.T., Akhmanova, A., et al. (2010). Pericentrosomal targeting of Rab6 secretory vesicles by Bicaudal-D-related protein 1 (BICDR-1) regulates neuritogenesis. EMBO J 29, 1637-1651.

Schmollinger, S., Strenkert, D., Offeddu, V., Nordhues, A., Sommer, F., and Schroda, M. (2012). A protocol for the identification of protein-protein interactions based on $15 \mathrm{~N}$ metabolic labeling, immunoprecipitation, quantitative mass spectrometry and affinity modulation. Journal of visualized experiments : JoVE.

Schornack, S., Fuchs, R., Huitema, E., Rothbauer, U., Lipka, V., and Kamoun, S. (2009). Protein mislocalization in plant cells using a GFP-binding chromobody. The Plant journal : for cell and molecular biology 60,744-754.

Schuldiner, M., Metz, J., Schmid, V., Denic, V., Rakwalska, M., Schmitt, H.D., Schwappach, B., and Weissman, J.S. (2008). The GET complex mediates insertion of tail-anchored proteins into the ER membrane. Cell 134, 634-645.

Schuske, K., Beg, A.A., and Jorgensen, E.M. (2004). The GABA nervous system in C. elegans. Trends in neurosciences 27, 407-414.

Schwappach, B., Stobrawa, S., Hechenberger, M., Steinmeyer, K., and Jentsch, T.J. (1998). Golgi localization and functionally important domains in the NH2 and COOH terminus of the yeast CLC putative chloride channel Geflp. J Biol Chem 273, 1511015118.

Schweizer, A., Fransen, J.A., Bachi, T., Ginsel, L., and Hauri, H.P. (1988). Identification, by a monoclonal antibody, of a $53-\mathrm{kD}$ protein associated with a tubulo-vesicular compartment at the cis-side of the Golgi apparatus. The Journal of cell biology 107, 1643-1653. 
Scott, J.W., Hawley, S.A., Green, K.A., Anis, M., Stewart, G., Scullion, G.A., Norman, D.G., and Hardie, D.G. (2004). CBS domains form energy-sensing modules whose binding of adenosine ligands is disrupted by disease mutations. The Journal of clinical investigation 113, 274-284.

Seaman, M.N. (2005). Recycle your receptors with retromer. Trends Cell Biol 15, 68-75. Selbach, M., and Mann, M. (2006). Protein interaction screening by quantitative immunoprecipitation combined with knockdown (QUICK). Nature methods 3, 981-983.

Serafini, T., Orci, L., Amherdt, M., Brunner, M., Kahn, R.A., and Rothman, J.E. (1991). ADP-ribosylation factor is a subunit of the coat of Golgi-derived COP-coated vesicles: a novel role for a GTP-binding protein. Cell 67, 239-253.

Setty, S.R., Shin, M.E., Yoshino, A., Marks, M.S., and Burd, C.G. (2003). Golgi recruitment of GRIP domain proteins by Arf-like GTPase 1 is regulated by Arf-like GTPase 3. Current biology : CB 13, 401-404.

Shanks, S.G., Carpp, L.N., Struthers, M.S., McCann, R.K., and Bryant, N.J. (2012). The Sec1/Munc18 protein Vps45 regulates cellular levels of its SNARE binding partners Tlg2 and Snc2 in Saccharomyces cerevisiae. PLoS One 7, e49628.

Shin, H.W., Kobayashi, H., Kitamura, M., Waguri, S., Suganuma, T., Uchiyama, Y., and Nakayama, K. (2005). Roles of ARFRP1 (ADP-ribosylation factor-related protein 1) in post-Golgi membrane trafficking. J Cell Sci 118, 4039-4048.

Short, B., Haas, A., and Barr, F.A. (2005). Golgins and GTPases, giving identity and structure to the Golgi apparatus. Biochimica et biophysica acta 1744, 383-395.

Simon, S.M. (2008). Golgi governance: the third way. Cell 133, 951-953.

Siniossoglou, S., and Pelham, H.R. (2001). An effector of Ypt6p binds the SNARE Tlg1p and mediates selective fusion of vesicles with late Golgi membranes. EMBO J 20, 59915998.

Smith, M.M., Lindstrom, J., and Merlie, J.P. (1987). Formation of the alpha-bungarotoxin binding site and assembly of the nicotinic acetylcholine receptor subunits occur in the endoplasmic reticulum. J Biol Chem 262, 4367-4376.

Sohn, K., Orci, L., Ravazzola, M., Amherdt, M., Bremser, M., Lottspeich, F., Fiedler, K., Helms, J.B., and Wieland, F.T. (1996). A major transmembrane protein of Golgi-derived COPI-coated vesicles involved in coatomer binding. The Journal of cell biology 135, 1239-1248.

Sonnichsen, B., De Renzis, S., Nielsen, E., Rietdorf, J., and Zerial, M. (2000). Distinct membrane domains on endosomes in the recycling pathway visualized by multicolor imaging of Rab4, Rab5, and Rab11. The Journal of cell biology 149, 901-914.

Spieth, J., and Blumenthal, T. (1985). The Caenorhabditis elegans vitellogenin gene family includes a gene encoding a distantly related protein. Mol Cell Biol 5, 2495-2501.

Stagg, S.M., LaPointe, P., and Balch, W.E. (2007). Structural design of cage and coat scaffolds that direct membrane traffic. Current opinion in structural biology 17, 221-228.

Stenmark, H. (2009). Rab GTPases as coordinators of vesicle traffic. Nature reviews Molecular cell biology 10, 513-525.

Sulston, J.E., Schierenberg, E., White, J.G., and Thomson, J.N. (1983). The embryonic cell lineage of the nematode Caenorhabditis elegans. Dev Biol 100, 64-119.

Sutton, R.B., Fasshauer, D., Jahn, R., and Brunger, A.T. (1998). Crystal structure of a SNARE complex involved in synaptic exocytosis at 2.4 A resolution. Nature 395, 347353. 
Szewczyk, N.J., Kozak, E., and Conley, C.A. (2003). Chemically defined medium and Caenorhabditis elegans. BMC biotechnology 3, 19.

Takahashi, S., Kubo, K., Waguri, S., Yabashi, A., Shin, H.W., Katoh, Y., and Nakayama, K. (2012). Rab11 regulates exocytosis of recycling vesicles at the plasma membrane. J Cell Sci 125, 4049-4057.

Takatsu, H., Katoh, Y., Shiba, Y., and Nakayama, K. (2001). Golgi-localizing, gammaadaptin ear homology domain, ADP-ribosylation factor-binding (GGA) proteins interact with acidic dileucine sequences within the cytoplasmic domains of sorting receptors through their Vps27p/Hrs/STAM (VHS) domains. J Biol Chem 276, 28541-28545.

Takizawa, P.A., and Malhotra, V. (1993). Coatomers and SNAREs in promoting membrane traffic. Cell 75, 593-596.

Tam, E.C.a.Y.Y.C. (2009). The endocytic pathway. Trafficking Inside Cells: Pathways, Mechanisms and Regulation.

Teasdale, R.D., and Jackson, M.R. (1996). Signal-mediated sorting of membrane proteins between the endoplasmic reticulum and the golgi apparatus. Annual review of cell and developmental biology 12, 27-54.

Teichert, U., Mechler, B., Muller, H., and Wolf, D.H. (1989). Lysosomal (vacuolar) proteinases of yeast are essential catalysts for protein degradation, differentiation, and cell survival. J Biol Chem 264, 16037-16045.

Terenzio, M., and Schiavo, G. (2010). The more, the better: the BICD family gets bigger. EMBO J 29, 1625-1626.

Thany, S.H., Lenaers, G., Raymond-Delpech, V., Sattelle, D.B., and Lapied, B. (2007). Exploring the pharmacological properties of insect nicotinic acetylcholine receptors. Trends in pharmacological sciences 28, 14-22.

Thyagarajan, K., Afshar, K., and Gonczy, P. (2011). Polarity mediates asymmetric trafficking of the Gbeta heterotrimeric G-protein subunit GPB-1 in C. elegans embryos. Development 138, 2773-2782.

Tokatlidis, K., Vial, S., Luciano, P., Vergnolle, M., and Clemence, S. (2000). Membrane protein import in yeast mitochondria. Biochemical Society transactions 28, 495-499.

Touroutine, D., Fox, R.M., Von Stetina, S.E., Burdina, A., Miller, D.M., 3rd, and Richmond, J.E. (2005). acr-16 encodes an essential subunit of the levamisole-resistant nicotinic receptor at the Caenorhabditis elegans neuromuscular junction. J Biol Chem 280, 27013-27021.

Town, M., Jean, G., Cherqui, S., Attard, M., Forestier, L., Whitmore, S.A., Callen, D.F., Gribouval, O., Broyer, M., Bates, G.P., et al. (1998). A novel gene encoding an integral membrane protein is mutated in nephropathic cystinosis. Nature genetics 18, 319-324.

Traub, L.M. (2003). Sorting it out: AP-2 and alternate clathrin adaptors in endocytic cargo selection. The Journal of cell biology 163, 203-208.

Treusch, S., Knuth, S., Slaugenhaupt, S.A., Goldin, E., Grant, B.D., and Fares, H. (2004). Caenorhabditis elegans functional orthologue of human protein h-mucolipin-1 is required for lysosome biogenesis. Proc Natl Acad Sci U S A 101, 4483-4488.

Trimble, W.S., Cowan, D.M., and Scheller, R.H. (1988). VAMP-1: a synaptic vesicleassociated integral membrane protein. Proc Natl Acad Sci U S A 85, 4538-4542.

Trinkle-Mulcahy, L., Boulon, S., Lam, Y.W., Urcia, R., Boisvert, F.M., Vandermoere, F., Morrice, N.A., Swift, S., Rothbauer, U., Leonhardt, H., et al. (2008). Identifying specific 
protein interaction partners using quantitative mass spectrometry and bead proteomes. The Journal of cell biology 183, 223-239.

van den Elsen, J.M., Kuntz, D.A., and Rose, D.R. (2001). Structure of Golgi alphamannosidase II: a target for inhibition of growth and metastasis of cancer cells. EMBO J 20, 3008-3017.

van Ijzendoorn, S.C. (2006). Recycling endosomes. J Cell Sci 119, 1679-1681.

van Koningsbruggen, S., de Haard, H., de Kievit, P., Dirks, R.W., van Remoortere, A., Groot, A.J., van Engelen, B.G., den Dunnen, J.T., Verrips, C.T., Frants, R.R., et al. (2003). Llama-derived phage display antibodies in the dissection of the human disease oculopharyngeal muscular dystrophy. Journal of immunological methods 279, 149-161.

Vida, T.A., and Emr, S.D. (1995). A new vital stain for visualizing vacuolar membrane dynamics and endocytosis in yeast. The Journal of cell biology 128, 779-792.

Von Stetina, S.E., Treinin, M., and Miller, D.M., 3rd (2006). The motor circuit. International review of neurobiology 69, 125-167.

Wach, A., Brachat, A., Pohlmann, R., and Philippsen, P. (1994). New heterologous modules for classical or PCR-based gene disruptions in Saccharomyces cerevisiae. Yeast 10, 1793-1808.

Wachter, A., and Schwappach, B. (2005). The yeast CLC chloride channel is proteolytically processed by the furin-like protease Kex $2 p$ in the first extracellular loop. FEBS Lett 579, 1149-1153.

Wanamaker, C.P., Christianson, J.C., and Green, W.N. (2003). Regulation of nicotinic acetylcholine receptor assembly. Annals of the New York Academy of Sciences 998, 6680 .

Wang, J.M., Zhang, L., Yao, Y., Viroonchatapan, N., Rothe, E., and Wang, Z.Z. (2002). A transmembrane motif governs the surface trafficking of nicotinic acetylcholine receptors. Nat Neurosci 5, 963-970.

Wang, X., Matteson, J., An, Y., Moyer, B., Yoo, J.S., Bannykh, S., Wilson, I.A., Riordan, J.R., and Balch, W.E. (2004). COPII-dependent export of cystic fibrosis transmembrane conductance regulator from the ER uses a di-acidic exit code. The Journal of cell biology 167, 65-74.

Wei, N., Serino, G., and Deng, X.W. (2008). The COP9 signalosome: more than a protease. Trends in biochemical sciences 33, 592-600.

Wessler, I., Kilbinger, H., Bittinger, F., Unger, R., and Kirkpatrick, C.J. (2003). The nonneuronal cholinergic system in humans: expression, function and pathophysiology. Life Sci 72, 2055-2061.

Westermann, B., and Neupert, W. (2000). Mitochondria-targeted green fluorescent proteins: convenient tools for the study of organelle biogenesis in Saccharomyces cerevisiae. Yeast 16, 1421-1427.

Wiedemann, N., Frazier, A.E., and Pfanner, N. (2004). The protein import machinery of mitochondria. J Biol Chem 279, 14473-14476.

Wilcox, C.A., Redding, K., Wright, R., and Fuller, R.S. (1992). Mutation of a tyrosine localization signal in the cytosolic tail of yeast Kex2 protease disrupts Golgi retention and results in default transport to the vacuole. Mol Biol Cell 3, 1353-1371.

Wu, Y., Yeh, F.L., Mao, F., and Chapman, E.R. (2009). Biophysical characterization of styryl dye-membrane interactions. Biophysical journal 97, 101-109. 
Yaffe, M.P., and Schatz, G. (1984). Two nuclear mutations that block mitochondrial protein import in yeast. Proc Natl Acad Sci U S A 81, 4819-4823.

Yeung, B.G., Phan, H.L., and Payne, G.S. (1999). Adaptor complex-independent clathrin function in yeast. Mol Biol Cell 10,3643-3659.

Yuan, D.S., Dancis, A., and Klausner, R.D. (1997). Restriction of copper export in Saccharomyces cerevisiae to a late Golgi or post-Golgi compartment in the secretory pathway. J Biol Chem 272, 25787-25793.

Yuan, D.S., Stearman, R., Dancis, A., Dunn, T., Beeler, T., and Klausner, R.D. (1995). The Menkes/Wilson disease gene homologue in yeast provides copper to a ceruloplasmin-like oxidase required for iron uptake. Proc Natl Acad Sci U S A 92, 26322636.

Zahn, C., Hommel, A., Lu, L., Hong, W., Walther, D.J., Florian, S., Joost, H.G., and Schurmann, A. (2006). Knockout of Arfrp1 leads to disruption of ARF-like1 (ARL1) targeting to the trans-Golgi in mouse embryos and HeLa cells. Molecular membrane biology 23, 475-485.

Zakrzewska, E., Perron, M., Laroche, A., and Pallotta, D. (2003). A role for GEA1 and GEA2 in the organization of the actin cytoskeleton in Saccharomyces cerevisiae. Genetics 165, 985-995.

Zeiler, M., Straube, W.L., Lundberg, E., Uhlen, M., and Mann, M. (2012). A Protein Epitope Signature Tag (PrEST) library allows SILAC-based absolute quantification and multiplexed determination of protein copy numbers in cell lines. Molecular \& cellular proteomics : MCP 11, O111009613.

Zerangue, N., Schwappach, B., Jan, Y.N., and Jan, L.Y. (1999). A new ER trafficking signal regulates the subunit stoichiometry of plasma membrane K(ATP) channels. Neuron 22, 537-548.

Zhang, B., and Zelhof, A.C. (2002). Amphiphysins: raising the BAR for synaptic vesicle recycling and membrane dynamics. Bin-Amphiphysin-Rvsp. Traffic 3, 452-460.

Zhang, Y., Grant, B., and Hirsh, D. (2001). RME-8, a conserved J-domain protein, is required for endocytosis in Caenorhabditis elegans. Mol Biol Cell 12, 2011-2021. 


\section{APPENDIX}

All used strains (bacteria E. coli, nematode C. elegans and yeast $S$. cerevisiae), oligonucleotides, plasmids, media, buffer solutions, materials, reagents and research equipment in this study are listed in the table XI.1.1 - XI.6.4 with description, source and manufacturer information provided.

\subsection{Strains of bacteria/yeast and $C$. elegans}

Table XI.1.1 Bacterial E. coli strains used in this study

\begin{tabular}{l|l|l}
\hline Strain & Description & Source \\
\hline E. coli OP50 & Feeding strains for C. elegans (uracil auxotroph) & (Brenner, 1974) \\
\hline E. coli DH5 $\alpha$ & Competent cells for molecular cloning & Invitrogen \\
\hline E. coli SLE1 & $\begin{array}{l}\text { Strains carrying pAG608 (orn-1 RNAi feeding vector) } \\
\text { for SILAC, [argA, lysA, mcrA, mcrB, IN (rrnD-rrnE) 1, } \\
\text { lambda-, rcn14::Tn10 (DE3 lysogen::lavUV5 promoter - }-\end{array}$ & $\begin{array}{l}\text { (Larance et al., } \\
\text { T7 polymerase] }\end{array}$ \\
\hline
\end{tabular}

Table XI.1.2 Yeast Saccharomyces cerevisiae strains used in this study

\begin{tabular}{|c|c|c|c|}
\hline Strain & Genotype & Background & Reference \\
\hline BY4741 & $\begin{array}{l}\text { MATa his } 3 \Delta 1, \text { leu } 2 \Delta 0, \text { met } 15 \Delta 0 \text {, } \\
\text { ura } 3 \Delta 0\end{array}$ & $\mathrm{~S} 288 \mathrm{C}$ & $\begin{array}{l}\text { (Brachmann et } \\
\text { al., 1998) }\end{array}$ \\
\hline$\Delta g m h 1 a$ & 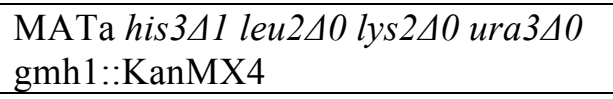 & S288C & Stefan Eimer \\
\hline$\Delta g m h 1:: \mathrm{Nat}^{\mathrm{R}}$ & MAT $\alpha \Delta g m h 1:: \mathrm{Nat}^{\mathrm{R}}$ & Y5563 & Stefan Eimer \\
\hline$\Delta g e f 1:: \mathrm{Nat}^{\mathrm{R}}$ & $\mathrm{BY} 4741 ; \Delta g e f 1:: \mathrm{Nat}^{\mathrm{R}}$ & BY4741 & $\begin{array}{l}\text { Matthieu S. } \\
\text { Peters }\end{array}$ \\
\hline$\Delta g m h 1 ; \Delta g e f 1$ & 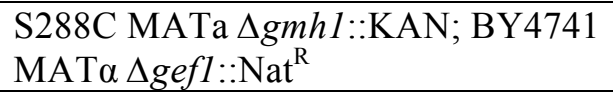 & & $\begin{array}{l}\text { Matthieu S. } \\
\text { Peters }\end{array}$ \\
\hline$\Delta p e p 4$ & BY4741; ppep 4::KanMX4 & BY4741 & EUROSCARF* \\
\hline$\Delta f e t 3$ & BY4741; $\Delta f e t 3:: K a n M X 4$ & BY4741 & $\begin{array}{l}\text { (Braun et al., } \\
2010)\end{array}$ \\
\hline 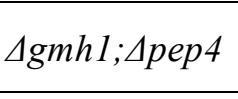 & $\begin{array}{l}\text { Y5563 MATa } \Delta g m h 1:: N A T ; \text { BY4741 } \\
\text { MATa } \text { Apep4::KanMX4 }\end{array}$ & & This study \\
\hline Anp1-RFP & 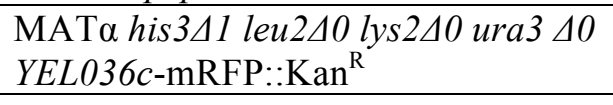 & BY4742 & (Huh et al., 2003) \\
\hline
\end{tabular}




\begin{tabular}{|c|c|c|c|}
\hline $\begin{array}{l}\text { Anp1-RFP; } \\
\Delta g m h 1:: \mathrm{Nat}^{\mathrm{R}}\end{array}$ & 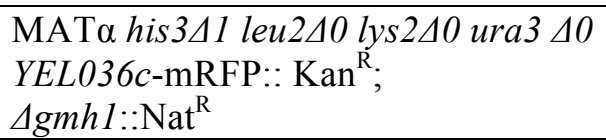 & Anp1-RFP & This study \\
\hline Chc1-RFP & $\begin{array}{l}\text { MAT } \alpha \text { his } 3 \Delta 1 \text { leu2 } 20 \text { lys } 2 \Delta 0 \text { ura3 } \Delta 0 \\
\text { YGL206c-mRFP::Kan }{ }^{\mathrm{R}}\end{array}$ & BY4742 & (Huh et al., 2003) \\
\hline $\begin{array}{l}\text { Chc1-RFP; } \\
\Delta g m h 1:: \mathrm{Nat}^{\mathrm{R}}\end{array}$ & $\begin{array}{l}\text { MAT } \alpha \text { his3 } 31 \text { leu2 } \Delta 0 \text { lys } 2 \Delta 0 \text { ura3 } \Delta 0 \\
\text { YGL206c-mRFP::Kan }{ }^{\mathrm{R}} ; \Delta \text { gmh } 1: \mathrm{Nat}^{\mathrm{R}}\end{array}$ & Chc1-RFP & This study \\
\hline Snf7-RFP & $\begin{array}{l}\text { MATa his } 3 \Delta 1 \text { leu } 2 \Delta 0 \text { lys } 2 \Delta 0 \text { ura } \Delta 0 \\
\text { YLR025w-mRFP::Kan }{ }^{\mathrm{R}}\end{array}$ & BY4742 & (Huh et al., 2003) \\
\hline $\begin{array}{l}\text { Snf7-RFP; } \\
\Delta g m h 1:: \mathrm{Nat}^{\mathrm{R}}\end{array}$ & $\begin{array}{l}\text { MAT } \alpha \text { his } 3 \Delta 1 \text { leu2 } \Delta 0 \text { lys } 2 \Delta 0 \text { ura3 } \Delta 0 \\
\text { YLR025w-mRFP ::Kan }{ }^{\mathrm{R}} \text {; } \\
\Delta g m h 1:: \mathrm{Nat}^{\mathrm{R}}\end{array}$ & Snf7RFP & This study \\
\hline
\end{tabular}

EUROSCARF*: http://web.uni-frankfurt.de/fb15/mikro/euroscarf

Matthieu S. Peters ${ }^{\S}$ : this stain was made by Matthieu S. Peters in collaboration with Blanche Schwappach and Stefan Eimer, 2011, Göttingen, Germany

Table XI.1.3 C. elegans strains used in this study

\begin{tabular}{|c|c|}
\hline Strain & Genotype \\
\hline Bristol & N2 (wild type) \\
\hline $\mathrm{X} 47$ & unc-50 (x47) III \\
\hline RB1539 & unc-50 (ok1847) III \\
\hline MF200 & ric-3 (hm9) IV \\
\hline CB1072 & unc-29 (e1072) I \\
\hline DH1336 & bIs34 [rme-8::GFP + rol-6 (su1006)] \\
\hline This study & unc-50 (x47) III; bIs34 [rme-8::GFP + rol-6 (su1006)] \\
\hline RT258 & unc-119 (ed3) III; pwIs50 [lmp-1::GFP + Cbr-unc-119(+)] \\
\hline This study & $\begin{array}{l}\text { unc-50 (x47) III; unc-119 (ed3) III; pwIs50 [lmp-1::GFP + } \\
\text { Cbr-unc-119(+)] }\end{array}$ \\
\hline CB5600 & him-8 (e1489) IV; ccIs4251 [pmyo-3::nls-gfp-lacZ; pmyo-3::mt-gfp] I \\
\hline This study & unc-50 (x47) III; ccIs4251 I; him-8 (e1489) IV \\
\hline GS1912 & arIs37 [pmyo-3::ssGFP] I; dpy-20 (e1282) IV \\
\hline This study & N2; arIs37 [pmyo-3::ssGFP] I; dpy-20 (e1282) IV; rol-6 (su1006) \\
\hline This study & unc-50 (x47) III; arIs37 [pmyo-3::ssGFP] I; dpy-20 (e1282) IV \\
\hline This study & $\begin{array}{l}\text { unc-50 (x47) III; arIs37 [pmyo-3::ssGFP] I; dpy-20 (e1282) IV; } \\
\text { rol-6 (su1006) }\end{array}$ \\
\hline Y54E10BR.2 & $\operatorname{Arfrpl}(\operatorname{tm} 2779) I$ \\
\hline
\end{tabular}




\begin{tabular}{|c|c|}
\hline KN555 & vps-35 (hu68) II \\
\hline VC2202 & vps-53 (ok2864) III \\
\hline NF1684 & $\operatorname{cog} c-3(k 181) I$ \\
\hline This study & $\begin{array}{l}\text { mIn1[dpy-10 (e128) mIs14 (myo-2::GFP)] II/vps-35 (hu68) II; } \\
\text { Arfrp1 (tm2779) I }\end{array}$ \\
\hline This study & ccIs4251 I/ $\operatorname{cog} c-3$ (k181) I; vps-35 (hu68) II \\
\hline This study & ccIs4251 I/ Arfrp1 (tm2779) I; vps-53 (ok2864) III \\
\hline This study & unc-50 (x47) III; gzIs20 [pmyo-3::unc-50::BirA*] \\
\hline This study & unc-50 (x47) III; gzIs21 [pmyo-3::GFP::BirA*] \\
\hline This study & 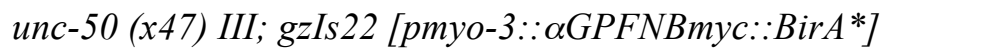 \\
\hline EG1653 & oxIs22 II [punc-49::unc-49::GFP + lin-15(+)] \\
\hline SJ4103 & zcIs 14 [pmyo-3::GFP (mito-matrix)] \\
\hline This study & oxIs22 II; sjEx [N2; InJ64 (pmyo-3::aGFPNBmyc::tagRFPt)] \\
\hline This study & 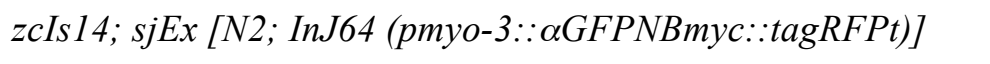 \\
\hline This study & 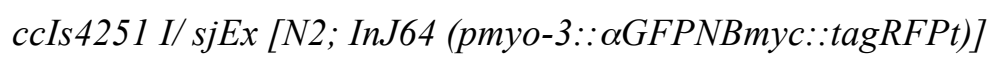 \\
\hline
\end{tabular}

BirA, biotin protein ligase in E. coli; BirA*, BirA (R118G); $\alpha$ GFPNB, anti-GFP-nanobody that contains the peptide sequences of a myc-tag. All mutant strains used in this study were outcrossed at least three times before use. Some nematode strains used in this study were provided by the CGC, which is funded by the NIH NCRR. arfrpl (tm2779) was supplied by the NBRP (Tokyo Women's Medical University School of Medicine). unc-50 (ok1847) was provided by Gene Knockout Project at Oklahoma Medical Research Foundation (OMRF), which is part of the international C. elegans Gene Knockout Consortium.

\subsection{Oligonucleotides and deletion alleles}

Table XI.2.1 DNA sequences of primers used in this study

\begin{tabular}{|c|c|c|}
\hline Primer & Sequence & Description \\
\hline oGQ2785 & GTGAAGTACGATCTAAAACTGTTG & $\begin{array}{l}\text { Forward primer for } g m h 1 \text {, that binds before } \\
\text { the promotor, for creating gmhl;pep } 4 \\
\text { double-deleted mutants using } \Delta g m h 1 \text { strains } \\
\text { without restriction enzyme sites }\end{array}$ \\
\hline oGQ2786 & ACGACCAGGAACACACCATTA & $\begin{array}{l}\text { Reverse primer for } g m h 1 \text {, that binds after the } \\
\text { terminator, for creating } g m h 1 \text {;pep } 4 \text { double- } \\
\text { deleted mutants using } \Delta g m h 1 \text { strains without } \\
\text { restriction enzyme sites }\end{array}$ \\
\hline
\end{tabular}




\begin{tabular}{|c|c|c|}
\hline oGQ617 & $\begin{array}{l}\text { GGGACTAGTTCTAGAATGTCATAT } \\
\text { TTGCCGACTTATTCC }\end{array}$ & $\begin{array}{l}\text { Forward primer of gmhl without promoter } \\
\text { that includes SpeI and } \mathrm{XbaI} \text { sites }\end{array}$ \\
\hline oGQ618 & $\begin{array}{l}\text { GGGGTCGACGGATCCTTAATAATT } \\
\text { GTAAAAGCTTAATTTCTTGGA }\end{array}$ & $\begin{array}{l}\text { Reverse primer of gmhl without promoter } \\
\text { that includes SalI and BamHI sites }\end{array}$ \\
\hline GefE287QF & TTGGAACAGATTGCTTCTG & $\begin{array}{l}\text { Forward primer for checking deletions of } \\
\text { gefl in yeast }\end{array}$ \\
\hline S618AR & $\begin{array}{l}\text { ACCATGCACAGCATATTCAGACGC } \\
\text { TGAGTC }\end{array}$ & $\begin{array}{l}\text { Reverse primer for checking deletions of } \\
\text { gefl in yeast }\end{array}$ \\
\hline oGQ1974 & TACACACTTTCGGCGGGTAT & External forward primer for arfrp1 (tm2779) \\
\hline oGQ1975 & ACAATCGGCAAGAAGCGGTG & External reverse primer for $\operatorname{arfrp} 1$ ( $\operatorname{tm} 2779)$ \\
\hline oGQ1976 & TTCGGCGGGTATCTGGGAGA & Internal forward primer for arfrp1 (tm2779) \\
\hline oGQ1977 & GGTTAGGAAATCATCACAGG & Internal reverse primer for arfrpl (tm2779) \\
\hline oGQ415 & $\begin{array}{l}\text { ATGTACGAAAATTCGGGGAATAC } \\
\text { GACCGACCAGGAGAAGTTTTTG }\end{array}$ & $\begin{array}{l}\text { Forward primer for vps-35 (hu68) (outside } \\
\text { deletion) }\end{array}$ \\
\hline oGQ416 & $\begin{array}{l}\text { TTAAGACGGAGGTTGCGGAAGTT } \\
\text { CAGGTTCTGAACGAATTGTTGT }\end{array}$ & $\begin{array}{l}\text { Reverse primer for vps-35 (hu68) (outside } \\
\text { deletion) }\end{array}$ \\
\hline oGQ417 & $\begin{array}{l}\text { GTGAGTTCCGACACATTTTTTTTA } \\
\text { ATTAGGGATGATTCTCAAAAA }\end{array}$ & $\begin{array}{l}\text { Forward primer for } v p s-35 \text { (hu68) (inside } \\
\text { deletion) }\end{array}$ \\
\hline oGQ418 & $\begin{array}{l}\text { ATTAAGATACAACTTCATTGGAAG } \\
\text { TTCTGCTAATTCAGCTGTCGA }\end{array}$ & $\begin{array}{l}\text { Reverse primer for } v p s-35 \text { (hu68) (inside } \\
\text { deletion) }\end{array}$ \\
\hline oGQ1654 & $\begin{array}{l}\text { CCAAGAAAGTATCAAAAACACTC } \\
\text { CAAATAG }\end{array}$ & $\begin{array}{l}\text { Forward primer vps-53 (ok2864) (inside } \\
\text { deletion) }\end{array}$ \\
\hline oGQ1655 & $\begin{array}{l}\text { GCTGATAATGAAGAAGGCGCATG } \\
\text { GTTGGAT }\end{array}$ & $\begin{array}{lllll}\text { Reverse primer } & v p s-53 & \text { (ok2864) } & \text { (inside } \\
\text { deletion) } & & & & \\
\end{array}$ \\
\hline oGQ1656 & $\begin{array}{l}\text { ATCAATGCATCAGGTTCACTGCTC } \\
\text { AACTTG }\end{array}$ & $\begin{array}{l}\text { Forward primer } v p s-53 \text { (ok2864) } \\
\text { deletion) }\end{array}$ \\
\hline oGQ1657 & $\begin{array}{l}\text { CGTATCGTAGATACCTTCATAATA } \\
\text { GAGTTA }\end{array}$ & $\begin{array}{l}\text { Reverse primer vps-53 (ok2864) (outside } \\
\text { deletion) }\end{array}$ \\
\hline oSE014 & $\begin{array}{l}\text { GGGGATCCACCGGTAGAAAAAAT } \\
\text { GTTACCGAGTACTTCAGTG }\end{array}$ & $\begin{array}{l}\text { Human gmhl forward primer that introduces } \\
\text { a BamHI and AgeI site upstream of the ATG }\end{array}$ \\
\hline oGQ2649 & $\begin{array}{l}\text { GGGCGGCCGCTTTCACTCTGTACT } \\
\text { TATAGAAAGAACAGAGAGT }\end{array}$ & 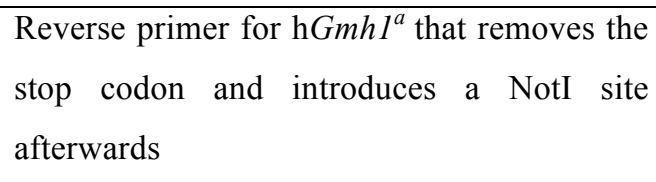 \\
\hline oSE016 & $\begin{array}{l}\text { GGGGATCCACCGGTAGAAAAAAT } \\
\text { GTCATATTTGCCGACTTATTCC }\end{array}$ & $\begin{array}{l}\text { Forward primer for gmhl that introduces a } \\
\text { BamHI and AgeI site upstream of the ATG }\end{array}$ \\
\hline oGQ2648 & $\begin{array}{l}\text { GGGCGGCCGCATAATTGTAAAAG } \\
\text { CTTAATTTCTTGGATAAGTCG }\end{array}$ & $\begin{array}{l}\text { Reverse primer for gmhl that removes the } \\
\text { stop codon and introduces a NotI site } \\
\text { afterwards }\end{array}$ \\
\hline oSE004 & СТTCTTCTTCСАCTTTTACCG & Forward primer for myo-3 promoter \\
\hline
\end{tabular}




\begin{tabular}{|c|c|c|}
\hline oGQ1395 & AACAGGTAGTTTTCCAGTAGTGCA & $\begin{array}{l}\text { Reverse primer matching the first exon in } \\
\text { GFP/YFP }\end{array}$ \\
\hline oGQ849 & $\begin{array}{l}\text { GGATCCAGGGTACCGGTAGCCAT } \\
\text { GGTTAAGCCGCAAGGAGGG }\end{array}$ & $\begin{array}{l}\text { Forward primer for tram-1 that starts at the } \\
\text { start ATG and introduces a BamHI, KpnI, } \\
\text { AgeI and NcoI site upstream }\end{array}$ \\
\hline oGQ850 & $\begin{array}{l}\text { CTCGAGAATTCAATTTTTCTTCTTC } \\
\text { GAATCGCTCTC }\end{array}$ & $\begin{array}{l}\text { Reverse primer for tram-1 that starts at the } \\
\text { stop codon and introduces a EcoRI and XhoI } \\
\text { site afterwards }\end{array}$ \\
\hline oGQ440 & AATTGCCAGATGAACAGCAGC & $\begin{array}{l}\text { Reverse sequencing primer for apt-9 (GGA } \\
\text { adaptor arf-binding) }\end{array}$ \\
\hline oGQ2238 & $\begin{array}{l}\text { GGCTTGCCTTCGCCCTCGGATGTG } \\
\text { C }\end{array}$ & $\begin{array}{l}\text { Reverse sequencing primer for TagRFP_T } \\
\text { binding at N-terminus }\end{array}$ \\
\hline oGQ2912 & $\begin{array}{l}\text { GGGGATCCGCGGTACCGCATATG } \\
\text { AAGGATAACACC GTGCCA }\end{array}$ & $\begin{array}{l}\text { Forward primer for E. coli birA that start at } \\
\text { the start ATG and introduces a BamHI, } \\
\text { KpnI, and NdeI site upstream }\end{array}$ \\
\hline oGQ2913 & $\begin{array}{l}\text { GAATTCTACCGGTGAGCGGCCGCC } \\
\text { TGCAGAACCACCTTTTTCTGCACT } \\
\text { ACGCAGGGA }\end{array}$ & $\begin{array}{l}\text { Reverse primer for E. coli birA that removes } \\
\text { the stop codon and introduces GGSA linker } \\
\text { and a NotI and AgeI site afterwards }\end{array}$ \\
\hline oGQ2914 & $\begin{array}{l}\text { CAGGCTGGCCGTGGTGGTCGGGG } \\
\text { TCGGAAATGGTTTTCG }\end{array}$ & $\begin{array}{l}\text { Forward mutagenesis primer for E. coli birA } \\
\text { that introduces the } \mathrm{R} 118 \mathrm{G} \text { mutation }\end{array}$ \\
\hline oGQ2915 & $\begin{array}{l}\text { CCATTTCCGACCCCGACCACCACG } \\
\text { GCCAGCCTGCTGATG }\end{array}$ & $\begin{array}{l}\text { Reverse mutagenesis primer for E. coli birA } \\
\text { that introduces the } \mathrm{R} 118 \mathrm{G} \text { mutation }\end{array}$ \\
\hline oGQ2943 & $\begin{array}{l}\text { GCGGTCTTGGTGGCGGCCGCGCG } \\
\text { GAGGCGGCTAGCGTGAAGGATAA } \\
\text { CACCGTGCCACT }\end{array}$ & $\begin{array}{l}\text { Forward primer for birA (R118G) pGEMT } \\
\text { that contains overlapped seq. of C-terminal } \\
\text { UNC-50 and introduces a NotI with linker } \\
\text { (GSGAS, 15nt) without the start codon of } \\
\text { birA for fusion at the downstream of UNC- } \\
50\end{array}$ \\
\hline oGQ2944 & $\begin{array}{l}\text { CCGAATTCTCATTTTTCTGCACTA } \\
\text { CGCAGGGA }\end{array}$ & $\begin{array}{l}\text { Reverse primer for birA (R118G) pGEMT } \\
\text { that introduces a EcoRI and the stop codon } \\
\text { of birA for fusion at the downstream of } \\
\text { UNC-50 }\end{array}$ \\
\hline oGQ3032 & $\begin{array}{l}\text { GCAAGCTTGGTACCGGATCCATGG } \\
\text { CC }\end{array}$ & $\begin{array}{l}\text { Forward primer for antiGFP-nanobody-myc- } \\
\text { tag that contains Met, Ala of the construct } \\
\text { GFPNB-Myc_pMAT }\end{array}$ \\
\hline oGQ3033 & GGGAATTCGCGGCCGCCCAGGTC & Reverse primer for antiGFP-nanobody-myc- \\
\hline
\end{tabular}


| CTCTTCGGA tag that introduces a NotI site behind the myc-tag

${ }^{a}: h G m h 1$ represents human $g m h 1$ which is $u n c-50$ like.

Table XI.2.2 List of deletion alleles used in C. elegans

\begin{tabular}{l|l|l|l|l|l|l}
\hline $\begin{array}{l}\text { Deletion } \\
\text { strain }\end{array}$ & $\begin{array}{l}\text { Size of } \\
\text { deletion } \\
\text { (bp) or } \\
\text { approx. }\end{array}$ & $\begin{array}{l}\text { Outside deletion } \\
\text { primers }\end{array}$ & $\begin{array}{l}\text { PCR band } \\
\text { sizes in } \\
\text { WT or } \\
\text { mutant }(\mathrm{m}) \\
\text { (bp) }\end{array}$ & $\begin{array}{l}\text { Inside deletion } \\
\text { primers }\end{array}$ & $\begin{array}{l}\text { PCR } \\
\text { band } \\
\text { sizes } \\
\text { in WT } \\
\text { (bp) }\end{array}$ & $\begin{array}{l}\text { Anneal. } \\
\text { temp./ } \\
\text { Extens. } \\
\text { time }\end{array}$ \\
\hline $\begin{array}{l}\text { Arfrp1 } \\
\text { (tm2779) }\end{array}$ & 404 & oGQ1974 & $493(\mathrm{~m})$ & oGQ1977 & 897 & $\begin{array}{l}50^{\circ} \mathrm{C} / \\
1: 00 \mathrm{~min}\end{array}$ \\
\hline $\begin{array}{l}\text { vps-53 } \\
\text { (ok2864) }\end{array}$ & 373 & oGQ1656/oGQ1657 & 1060 & oGQ1654/oGQ1657 & 855 & $\begin{array}{l}51^{\circ} \mathrm{C} / \\
1: 30 \mathrm{~min}\end{array}$ \\
\hline $\begin{array}{l}\text { vps-53 } \\
\text { (ok2864) }\end{array}$ & 314 & oGQ1657 & $541(\mathrm{~m})$ & oGQ1654 & 855 & $\begin{array}{l}51^{\circ} \mathrm{C} / \\
1: 30 \mathrm{~min}\end{array}$ \\
\hline $\begin{array}{l}\text { vps-53 } \\
\text { (ok2864) }\end{array}$ & - & & $\begin{array}{l}\text { no bands } \\
(\mathrm{m})\end{array}$ & oGQ1654/oGQ1655 & 334 & $\begin{array}{l}59^{\circ} \mathrm{C} / \\
2: 00 \mathrm{~min}\end{array}$ \\
\hline $\begin{array}{l}\text { vps-35 } \\
\text { (hu68) }\end{array}$ & - & oGQ415 & $380(\mathrm{~m})$ & oGQ416 & 2889 & $\begin{array}{l}68^{\circ} \mathrm{C} / \\
2: 30 \mathrm{~min}\end{array}$ \\
\hline $\begin{array}{l}\text { vps-35 } \\
\text { (hu68) }\end{array}$ & - & oGQ415 & $\begin{array}{l}\text { no bands } \\
(\mathrm{m})\end{array}$ & oGQ418 & 2137 & $\begin{array}{l}64^{\circ} \mathrm{C} / \\
2: 30 \mathrm{~min}\end{array}$ \\
\hline
\end{tabular}

Table XI.2.3 Polymerase chain reaction conditions in yeast cells

\begin{tabular}{|c|c|c|c|c|}
\hline $\begin{array}{l}\text { Reagents for } \\
\text { master mix }\end{array}$ & $\begin{array}{l}(\mu \mathrm{l}) \text { vol. in } 50 \\
\mu l \text { per reaction }\end{array}$ & $\begin{array}{l}\text { Program of PCR } \\
\text { (cycle in order) }\end{array}$ & $\begin{array}{l}\text { Amplified with } \\
\text { the primer pairs } \\
\text { (Forward / Reverse) }\end{array}$ & $\begin{array}{l}\text { Predicted } \\
\text { sizes (bp) } \\
\text { approx. }\end{array}$ \\
\hline 10X PCR Buffer & 5 & (1) $3 \mathrm{~m}, 95^{\circ} \mathrm{C}$ & oGQ617/oGQ618 & 850 \\
\hline $2.5 \mathrm{mM}$ dNTP & 4 & (2) $20 \mathrm{~s}, 95^{\circ} \mathrm{C}$ & GefE287QF/S618AR & 1100 \\
\hline $10 \mu \mathrm{M}(\mathrm{F})$ primer & 2 & (3) $1 \mathrm{~m} 30 \mathrm{~s}, 51^{\circ} \mathrm{C}$ & \multicolumn{2}{|c|}{ for checking deletions (gmhl, gefl) } \\
\hline $10 \mu \mathrm{M}(\mathrm{R})$ primer & 2 & (4) $1 \mathrm{~m}, 72^{\circ} \mathrm{C}$ & oGQ2785/oGQ2786 & 1800 \\
\hline Taq-Polymerase & 1 & (5) 35 cycles & \multirow{2}{*}{\multicolumn{2}{|c|}{$\begin{array}{l}\text { Template*: } \Delta g m h 1:: \mathrm{Nat}^{\mathrm{R}} \\
\text { for making } \Delta g m h 1 ; \Delta \text { pep } 4\end{array}$}} \\
\hline (2.5 unit, Roche) & & from (2) to (4) & & \\
\hline $\mathrm{ddH}_{2} \mathrm{O}$ & 35 & (6) $5 \mathrm{~m}, 72^{\circ} \mathrm{C}$ & oGQ617/oGQ618 & 850 \\
\hline $\begin{array}{l}\text { Template* (Chen et } \\
\text { al.) }\end{array}$ & $1(20-80 \mathrm{ng})$ & (7) keep at $4^{\circ} \mathrm{C}$ & \multicolumn{2}{|c|}{ for double-checking gmh1 mutants } \\
\hline
\end{tabular}

Template*, genomic DNAs purified were used for checking deletion mutants and also creating $\Delta g m h 1:: \mathrm{Nat}^{\mathrm{R}}$ knockout strains in order to be transformed in various strains which were $\Delta$ pep $4:: \mathrm{Kan}^{\mathrm{R}}$, Anp1-RFP::Kan ${ }^{\mathrm{R}}$, Chc1-RFP:: $\operatorname{Kan}^{\mathrm{R}}$ and Snf7-RFP::Kan ${ }^{\mathrm{R}}$ by PCR-based drug resistance cassettes for gene deletions (Goldstein and McCusker, 1999; Wach et al., 1994). 


\subsection{Plasmids and injection constructs}

Table XI.3.1 List of backbone vectors used in this study

\begin{tabular}{l|c|l|l}
\hline Backbone vectors & $\begin{array}{l}\text { Resistance } \\
\text { Gene }\end{array}$ & Description & Source \\
\hline pBluescript II SK $(+)$ & $A^{\mathrm{r}} \mathrm{p}^{\mathrm{r}}$ & $\begin{array}{l}\text { Entry vector for cloning and used } \\
\text { for injection mixtures }\end{array}$ & Stratagene \\
\hline pGEMT & $\mathrm{Amp}^{\mathrm{r}}$ & Entry vector for TA cloning & Promega \\
\hline $\begin{array}{l}\text { pPD115.62 } \\
(\text { pmyo-3::gfp })\end{array}$ & $\mathrm{Amp}^{\mathrm{r}}$ & $\begin{array}{l}\text { Muscle expression vector for } C . \\
\text { elegans contains } \text { unc-54 3'UTR }\end{array}$ & $\begin{array}{l}\text { Addgene, Andrew } \\
\text { Fire vector kit }\end{array}$ \\
\hline
\end{tabular}

Table XI.3.2 List of genes of interest in entry vectors

\begin{tabular}{|c|c|c|c|}
\hline $\begin{array}{l}\text { Genes of interest } \\
\text { in entry vectors }\end{array}$ & $\begin{array}{l}\text { Resistance } \\
\text { Gene } \\
\end{array}$ & Cloning Strategy & Source \\
\hline pGEMT gmhl & $A m p^{r}$ & $\begin{array}{l}\text { PCR with oSE016/oGQ2648 and } \\
\text { digested with AgeI/NotI }\end{array}$ & Cloned in this study \\
\hline pGEMT $h G m h 1$ & $A m p^{r}$ & $\begin{array}{l}\text { PCR with oSE014/oGQ2649 and } \\
\text { digested with AgeI/NotI }\end{array}$ & Cloned in this study \\
\hline pGEMT tram-1 & $A m p^{r}$ & - & $\begin{array}{l}\text { Kindly provided by } \\
\text { Sabine Koenig }\end{array}$ \\
\hline pGEMT BirA & $A m p^{r}$ & $\begin{array}{l}\text { Mutagenesis PCR with } \\
\text { oGQ2914/oGQ2915 for E. coli } \\
\text { birA (R118G) pGEMT }\end{array}$ & $\begin{array}{l}\text { Kindly provided by } \\
\text { Sabine Koenig }\end{array}$ \\
\hline pGEMT BirA* & $A m p^{r}$ & $\begin{array}{l}\text { PCR with oGQ2943/oGQ2944 } \\
\text { and digested with NotI/EcoRI }\end{array}$ & Cloned in this study \\
\hline pMAT $\alpha G F P N B m y c$ & $A m p^{r}$ & $\begin{array}{l}\text { PCR with oGQ3032/oGQ3033 } \\
\text { and digested with KpnI/NotI }\end{array}$ & $\begin{array}{l}\text { Kindly provided by } \\
\text { Nora Wender }\end{array}$ \\
\hline
\end{tabular}

$\alpha$ GFPNB-myc, anti-GFP nanobodies that contain a polypeptide protein myc-tag.

Table XI.3.3 List of genes of interest in injection vectors

\begin{tabular}{l|l|l|l}
\hline $\begin{array}{l}\text { Genes of interest in injection } \\
\text { vectors }\end{array}$ & $\begin{array}{l}\text { Resistan } \\
\text { ce } \\
\text { Gene }\end{array}$ & Description & Source \\
\hline$p R F 4-$ rol-6 & Amp ${ }^{\mathrm{r}}$ & $\begin{array}{l}\text { Co-injection marker, } \\
\text { rol-6 (su1006) }\end{array}$ & (Mello and Fire, 1995) \\
\hline
\end{tabular}




\begin{tabular}{|c|c|c|c|}
\hline$p t t x-3: \because g f p$ & $\mathrm{Amp}^{\mathrm{r}}$ & $\begin{array}{l}\text { Co-injection marker, } g f p \\
\text { expression in head as a } \\
\text { pair of AIY inter-neuron }\end{array}$ & (Hobert et al., 1997) \\
\hline$p t t x-3:: r f p$ & $A m p^{r}$ & - & $\begin{array}{l}\text { Kindly provided by } \\
\text { Sabine Koenig }\end{array}$ \\
\hline pmyo-3::tom $70-c f p$ & $A m p^{r}$ & $\begin{array}{l}\text { Mitochondrial outer } \\
\text { membrane marker in } C \text {. } \\
\text { elegans muscle cells }\end{array}$ & $\begin{array}{l}\text { Kindly provided by } \\
\text { Alexander van der } \\
\text { Bliek }\end{array}$ \\
\hline pmyo-3::unc-50-yfp & $A m p^{r}$ & - & $\begin{array}{l}\text { Kindly provided by } \\
\text { Stefan Eimer }\end{array}$ \\
\hline pmyo-3::hGmh1-yfp & $\mathrm{Amp}^{\mathrm{r}}$ & $\begin{array}{l}\text { PCR with oSE014/ } \\
\text { oGQ2649; digested with } \\
\text { AgeI/NotI }\end{array}$ & Cloned in this study \\
\hline pmyo-3::gmh1-yfp & $A m p^{r}$ & $\begin{array}{l}\text { PCR with oSE016/ } \\
\text { oGQ2648; digested with } \\
\text { AgeI/NotI }\end{array}$ & Cloned in this study \\
\hline pmyo-3::mans-tagRFP & $\mathrm{Amp}^{\mathrm{r}}$ & - & $\begin{array}{l}\text { Kindly provided by } \\
\text { Ling Luo }\end{array}$ \\
\hline pmyo-3::ctns-1-yfp & $A m p^{r}$ & - & $\begin{array}{l}\text { Kindly provided by } \\
\text { Ling Luo }\end{array}$ \\
\hline pmyo-3::gfp-2xfyve & $A m p^{r}$ & - & (Luo et al., 2011) \\
\hline pmyo-3::yfp-rab-5 & $A m p^{r}$ & - & $\begin{array}{l}\text { Kindly provided by } \\
\text { Marija Sumakovic }\end{array}$ \\
\hline pmyo-3::mcherry-rab-7 & $\mathrm{Amp}^{\mathrm{r}}$ & - & $\begin{array}{l}\text { Kindly provided by } \\
\text { Sabine Koenig }\end{array}$ \\
\hline pmyo-3::mcherry- $\varepsilon$ Cop & $\mathrm{Amp}^{\mathrm{r}}$ & - & $\begin{array}{l}\text { Kindly provided by } \\
\text { Ling Luo }\end{array}$ \\
\hline prab-3::tagRFP-rab-11.1 & $A m p^{r}$ & - & $\begin{array}{l}\text { Kindly provided by } \\
\text { Nikhil Sasidharan }\end{array}$ \\
\hline pmyo-3::tagRFP-rab-11.1 & $\mathrm{Amp}^{\mathrm{r}}$ & $\begin{array}{l}\text { Subcloned from prab- } \\
3: \because \operatorname{tag} R F P-r a b-11.1 \\
\text { digested with KpnI/ } \\
\text { HindIII }\end{array}$ & Cloned in this study \\
\hline prab-3::mcherry-apt-9 & $A m p^{r}$ & - & $\begin{array}{l}\text { Kindly provided by } \\
\text { Mandy Hannemann }\end{array}$ \\
\hline pmyo-3::tagRFPt-apt-9 & $A m p^{r}$ & $\begin{array}{l}\text { Subcloned from prab- } \\
3:: \text { mcherry-apt- } 9 \text {; } \\
\text { digested with NotI/ } \\
\text { XhoI }\end{array}$ & Cloned in this study \\
\hline
\end{tabular}




\begin{tabular}{|c|c|c|c|}
\hline pmyo-3::gfp-tram-1 & $\mathrm{Amp}^{\mathrm{r}}$ & $\begin{array}{l}\text { Subcloned from } \\
\text { pGEMT tram-1 with } \\
\text { oGQ849/oGQ850; } \\
\text { digested with AgeI/ } \\
\text { EcoRI }\end{array}$ & Cloned in this study \\
\hline pmyo-3::unc-50-BirA* & $A m p^{r}$ & $\begin{array}{l}\text { Subcloned from } \\
\text { pGEMT BirA* with } \\
\text { NotI/EcoRI }\end{array}$ & Cloned in this study \\
\hline pmyo-3::gfp-BirA* & $A m p^{r}$ & $\begin{array}{l}\text { Subcloned from } \\
\text { pmyo3::gfp-rab-10; } \\
\text { digested with KpnI/ } \\
\text { NotI }\end{array}$ & Cloned in this study \\
\hline 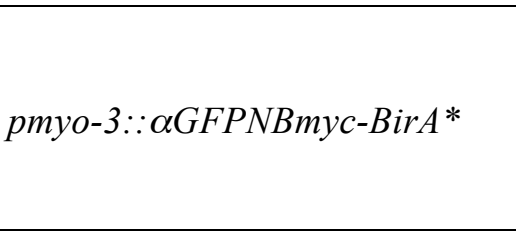 & $A m p^{r}$ & $\begin{array}{l}\text { Subcloned from } \\
\alpha G F P N B-m y c-p M A T \\
\text { with oGQ3032/oGQ30 } \\
33 \text {; digested with KpnI/ } \\
\text { NotI }\end{array}$ & Cloned in this study \\
\hline pmyo-3:: $\alpha G F P N B m y c-t a g R F P t$ & $A m p^{r}$ & $\begin{array}{l}\text { Subcloned from pmyo- } \\
3:: \text { moesin-tagRFPt; } \\
\text { digested with NotI/ } \\
\text { EcoRI }\end{array}$ & Cloned in this study \\
\hline
\end{tabular}

$\mathrm{Amp}^{\mathrm{r}}$, ampicillin; BirA, biotin protein ligase in E. coli; BirA*, BirA(R118G); $\alpha \mathrm{GFPNB}$, anti-GFP nanobody that contains the peptide sequences of c-myc-tag.

Table XI.3.4 List of transgenic lines of extrachromosomal arrays (Ex.)

\begin{tabular}{|c|c|c|}
\hline Ex. Name & Plasmids of interest & Injection markers \\
\hline InJ13 & $3 \mathrm{ng} / \mathrm{\mu l}$ pmyo-3::unc-50-yfp & $40 n g / \mu l$ prab-3::gfp \\
\hline InJ14 & $2 \mathrm{ng} / \mathrm{\mu l}$ pmyo-3::unc-50-yfp & $40 \mathrm{ng} / \mu \mathrm{l}$ pRF4 (rol-6 (su1006)) \\
\hline InJ16 & $2 \mathrm{ng} / \mathrm{\mu l}$ pmyo-3::unc-50-yfp & $\begin{array}{l}20 n g / \mu l p t t x-3:: g f p \\
40 n g / \mu l p R F 4(\text { rol-6 (su1006)) }\end{array}$ \\
\hline InJ18 & $\begin{array}{l}3 \mathrm{ng} / \mu \mathrm{l} \text { pmyo-3::gmh1 (human)-yfp; } \\
1 \mathrm{ng} / \mu \mathrm{l} \text { pmyo-3::mans-tagRFP }\end{array}$ & $\begin{array}{l}20 n g / \mu l p t t x-3:: g f p \\
40 n g / \mu l p R F 4(\text { rol-6 (su1006)) }\end{array}$ \\
\hline InJ19 & $\begin{array}{l}3 \mathrm{ng} / \mu \mathrm{l} \text { pmyo-3::gmh1 (yeast)-yfp; } \\
1 \mathrm{ng} / \mu \mathrm{l} \text { pmyo-3::mans-tagRFP}\end{array}$ & $\begin{array}{l}20 n g / \mu l p t t x-3:: g f p \\
40 n g / \mu l p R F 4(\text { rol-6 (su1006)) }\end{array}$ \\
\hline InJ20 & $\begin{array}{l}3 \mathrm{ng} / \mu \mathrm{l} \text { pmyo-3::unc-50-yfp; } \\
1 \mathrm{ng} / \mu \mathrm{l} \text { pmyo-3::mans-tagRFP }\end{array}$ & $\begin{array}{l}20 n g / \mu l p t t x-3:: g f p \\
40 n g / \mu l p R F 4(\text { rol-6 (su1006)) }\end{array}$ \\
\hline InJ40 & 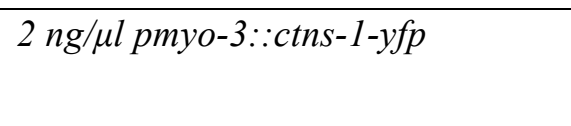 & $\begin{array}{l}40 \mathrm{ng} / \mu \mathrm{l} \text { pRF4 (rol-6 (su1006)); } \\
5 \mathrm{ng} / \mu \mathrm{l} \text { pmyo-3::tom-70-CFP }\end{array}$ \\
\hline
\end{tabular}




\begin{tabular}{|c|c|c|}
\hline InJ42 & $2 n g / \mu l$ pmyo-3::gfp-2xfyve & $\begin{array}{l}40 \mathrm{ng} / \mu \mathrm{l} \mathrm{pRF4}(\text { rol-6 (su1006)), } \\
5 \mathrm{ng} / \mu \mathrm{l} \text { pmyo-3::tom-70-CFP }\end{array}$ \\
\hline InJ43 & $2 n g / \mu l$ pmyo-3::yfp-rab-5 & $\begin{array}{l}40 \mathrm{ng} / \mu \mathrm{l} \text { pRF4 (rol-6 (su1006)), } \\
5 \mathrm{ng} / \mu \mathrm{l} \text { pmyo-3::tom-70-CFP }\end{array}$ \\
\hline InJ47 & $10 \mathrm{ng} / \mathrm{ll}$ pmyo-3::mcherry-eCop & $\begin{array}{l}40 \mathrm{ng} / \mu \mathrm{l} \text { pRF4 (rol-6 (su1006)), } \\
5 \mathrm{ng} / \mu \mathrm{l} \text { pmyo-3::tom-70-CFP }\end{array}$ \\
\hline InJ49 & $5 \mathrm{ng} / \mu \mathrm{l}$ pmyo-3::tagRFP-rab-11.1 & $\begin{array}{l}40 \mathrm{ng} / \mu \mathrm{l} \text { pRF4 (rol-6 (su1006)), } \\
5 \mathrm{ng} / \mu \mathrm{l} \text { pmyo-3::tom-70-CFP; } \\
20 \mathrm{ng} / \mu \mathrm{l} \text { pttx-3::gfp }\end{array}$ \\
\hline SK48 & $2 \mathrm{ng} / \mu \mathrm{l}$ pmyo-3:: mcherry-rab-7 & $\begin{array}{l}40 \mathrm{ng} / \mu \mathrm{l} \text { pRF4 (rol-6 (su1006)), } \\
5 \mathrm{ng} / \mu \mathrm{l} \text { pmyo-3::tom-70-CFP }\end{array}$ \\
\hline InJ51 & $5 \mathrm{ng} / \mu \mathrm{l}$ pmyo-3::tagRFP-apt-9 & $\begin{array}{l}40 \mathrm{ng} / \mu \mathrm{l} \text { pRF4 (rol-6 (su1006)), } \\
5 \mathrm{ng} / \mu \mathrm{l} \text { pmyo-3::tom-70-CFP; } \\
20 \mathrm{ng} / \mu \mathrm{l} \text { pttx-3::gfp }\end{array}$ \\
\hline InJ53 & $3 \mathrm{ng} / \mu \mathrm{l}$ pmyo-3::mans-tagRFP & $\begin{array}{l}40 \mathrm{ng} / \mu \mathrm{l} \text { pRF4 (rol-6 (su1006)), } \\
5 \mathrm{ng} / \mu \mathrm{l} \text { pmyo-3::tom-70-CFP; } \\
20 \mathrm{ng} / \mu \mathrm{l} \text { pttx-3::gfp }\end{array}$ \\
\hline InJ54 & $5 \mathrm{ng} /$ / l pmyo-3::gfp-tram-1 & 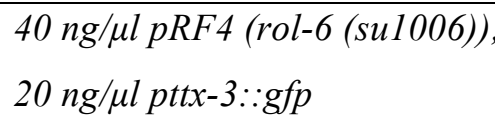 \\
\hline InJ55 & $\begin{array}{l}3 \mathrm{ng} / \mu \mathrm{l} \text { pmyo-3::unc-50-yfp; } \\
5 \mathrm{ng} / \mu \mathrm{l} \text { pmyo-3::tagRFP-apt-9}\end{array}$ & $\begin{array}{l}40 \mathrm{ng} / \mu \mathrm{l} \text { pRF4 (rol-6 (su1006)), } \\
5 \mathrm{ng} / \mu \mathrm{l} \text { pmyo-3::tom-70-CFP; } \\
20 \mathrm{ng} / \mu \mathrm{l} \text { pttx-3::gfp }\end{array}$ \\
\hline InJ60 & $\begin{array}{l}10 \mathrm{ng} / \mu \mathrm{l} \text { pmyo-3::unc-50-BirA* } \\
\text { unc-50 (x47)-background }\end{array}$ & $\begin{array}{l}40 \mathrm{ng} / \mu \mathrm{l} \text { pRF4 (rol-6 (su1006)), } \\
20 \mathrm{ng} / \mu \mathrm{l} \text { pttx-3: }: \mathrm{gfp}\end{array}$ \\
\hline InJ61 & $\begin{array}{l}10 \mathrm{ng} / \text { / l pmyo-3::gfp-BirA* } \\
\text { unc-50 (x47)-background }\end{array}$ & $\begin{array}{l}40 \mathrm{ng} / \mu \mathrm{l} \text { pRF4 (rol-6 (su1006)), } \\
20 \mathrm{ng} / \mu \mathrm{l} \text { pttx-3::gfp }\end{array}$ \\
\hline InJ62 & 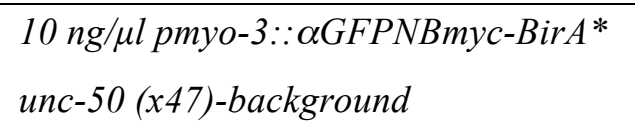 & $\begin{array}{l}40 \mathrm{ng} / \mu \mathrm{l} p \mathrm{RF} 4(\text { rol-6 (su1006)), } \\
20 \mathrm{ng} / \mu \mathrm{l} \text { pttx-3::gfp }\end{array}$ \\
\hline InJ63 & 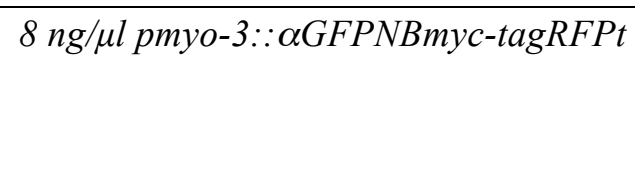 & $\begin{array}{l}40 \mathrm{ng} / \mu \mathrm{l} \text { pRF4 (rol-6 (su1006)), } \\
8 \mathrm{ng} / \mu \mathrm{l} \text { pmyo-3::tom-70-CFP; } \\
20 \mathrm{ng} / \mu \mathrm{l} \text { pttx-3::gfp }\end{array}$ \\
\hline InJ64 & 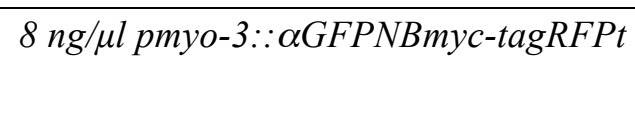 & $\begin{array}{l}40 \mathrm{ng} / \mu \mathrm{l} \text { pRF4 (rol-6 (su1006)), } \\
20 \mathrm{ng} / \mu \mathrm{l} \text { pttx-3::gfp }\end{array}$ \\
\hline
\end{tabular}




\begin{tabular}{|c|c|c|}
\hline InJ65 & 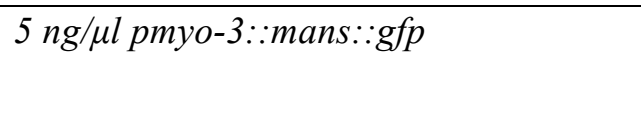 & 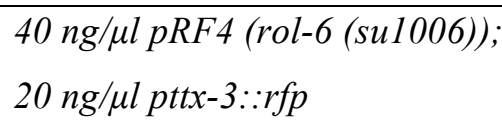 \\
\hline InJ66 & 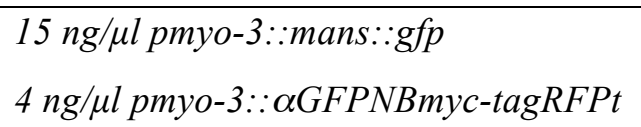 & 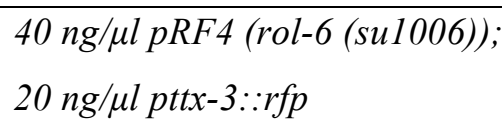 \\
\hline InJ67 & 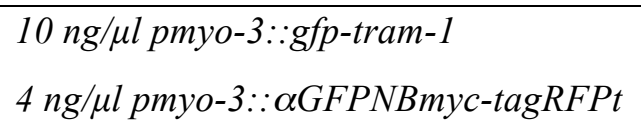 & $\begin{array}{l}40 \mathrm{ng} / \mu \mathrm{l} \mathrm{pRF4}(\text { rol-6 (su1006)); } \\
20 \mathrm{ng} / \mu \mathrm{l} \mathrm{pttx-3::rfp}\end{array}$ \\
\hline InJ68 & 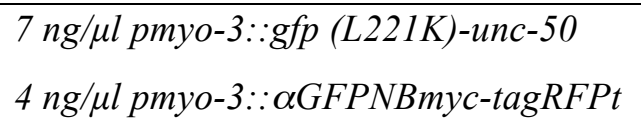 & $\begin{array}{l}40 \mathrm{ng} / \mu \mathrm{l} \text { pRF }(\text { rol-6 (su1006)); } \\
20 \mathrm{ng} / \mu \mathrm{l} \mathrm{pttx-3::rfp}\end{array}$ \\
\hline
\end{tabular}

Mans, alpha-mannosidase II; BirA, biotin protein ligase in E. coli; BirA*, BirA (R118G).

Table XI.3.5 List of plasmids used in yeast experiments

\begin{tabular}{|c|c|c|c|}
\hline Plasmid & $\begin{array}{l}\text { Selective } \\
\text { Marker }\end{array}$ & Description & Reference \\
\hline pAG25 & $\begin{array}{c}\mathrm{Nat}^{\mathrm{R}} \\
\text { (natMX4) }\end{array}$ & $\begin{array}{l}\text { Template for gene deletions by } \\
\text { drug resistance cassettes }\end{array}$ & $\begin{array}{l}\text { (Goldstein and } \\
\text { McCusker, 1999; } \\
\text { Wach et al., 1994) }\end{array}$ \\
\hline p416MET25 & URA3 & Expression vector in yeast & $\begin{array}{l}\text { (Mumberg et al., } \\
\text { 1994) }\end{array}$ \\
\hline $\begin{array}{l}\text { p416MET25-GFP- } \\
\text { GEF1 }\end{array}$ & URA3 & N-terminally tagged-GFP & (Braun et al., 2010) \\
\hline $\begin{array}{l}\text { GEF1-GFP- } \\
\text { p416MET25 }\end{array}$ & URA3 & C-terminally tagged-GFP & $\begin{array}{l}\text { (Schwappach et al., } \\
\text { 1998) }\end{array}$ \\
\hline p416 NHX1-GFP & URA3 & Sodium/proton exchanger & $\begin{array}{l}\text { (Bowers et al., } \\
\text { 2000) }\end{array}$ \\
\hline p415 Redstar-GEF1 & LEU2 & Tandem dimer-tagged & (Knop et al., 2002) \\
\hline pYESmt-GFP & URA3 & $\begin{array}{l}\text { pre } \mathrm{Su} 9 \text { as Mitochondria } \\
\text { markers }(2 \% \text { galactose })\end{array}$ & $\begin{array}{l}\text { (Westermann and } \\
\text { Neupert, 2000) }\end{array}$ \\
\hline pRS315 GFP-SNC2 & URA3 & $v$-SNARE & $\begin{array}{l}\text { (Protopopov et al., } \\
\text { 1993) }\end{array}$ \\
\hline pRS315 GFP-TLG2 & URA3 & $t$-SNARE & $\begin{array}{l}\text { (Schuldiner et al., } \\
\text { 2008) }\end{array}$ \\
\hline TLG2-mCherry & URA3 & $t$-SNARE & $\begin{array}{l}\text { Schwappach lab } \\
\text { (Schuldiner et al., } \\
\text { 2008) }\end{array}$ \\
\hline
\end{tabular}

\subsection{Media}


Table XI.4.1 List of media used in this study

Synthetic complete (SC) medium

$6.7 \mathrm{~g}$ Yeast Nitrogen Base w/o Amino Acids

$0.65 \mathrm{~g}$ Amino acid powders w/o URA

$50 \mathrm{ml} \mathrm{40 \%} \mathrm{Glucose}$

$10 \mathrm{ml}$ 100x Uracil (2 g/L)

Up to 1 Liter $d_{d d} \mathrm{H}_{2} \mathrm{O}$ and use sterile filter

Yeast extract peptone-dextrose (YPAD) medium

$10 \mathrm{~g}$ Yeast Extract

20 g Bacto-Pepton

Autoclave and add below ones after cooling down

$10 \mathrm{ml} \mathrm{100x}$ Adenine (4 g/L)

$50 \mathrm{ml} 40 \%$ Glucose

Up to 1 Liter $\mathrm{ddH}_{2} \mathrm{O}$

SC-URA medium

$6.7 \mathrm{~g}$ Yeast Nitrogen Base w/o Amino Acids

$0.65 \mathrm{~g}$ CSM amino acid powders w/o URA

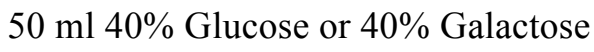

Up to 1 Liter $\mathrm{ddH}_{2} \mathrm{O}$ and use sterile filter

YPAD plate

$10 \mathrm{~g}$ Yeast Extract

20 g Bacto-Pepton

15 g Bacto-Agar

Autoclave and add below ones after cooling down

$10 \mathrm{ml} \mathrm{100x}$ Adenine (4 g/L)

$50 \mathrm{ml} \mathrm{40 \%} \mathrm{Glucose}$

Up to 1 Liter $\mathrm{ddH}_{2} \mathrm{O}$

\section{SC-URA or SC-URA/LEU plate}

$6.7 \mathrm{~g}$ Yeast Nitrogen Base w/o Amino Acids

$15 \mathrm{~g}$ Bacto-Agar

Autoclave and add below ones after cooling down

0.65 g CSM-ADE-HIS-LEU-MET-TRP-URA

$4 \mathrm{ml} \mathrm{100x}$ Adenine (4 g/L)

$2 \mathrm{ml} \mathrm{100x}$ Histidine (2 $\mathrm{g} / \mathrm{L})$

$2 \mathrm{ml}$ 100x Methionine (2 g/L)

$5 \mathrm{ml} 100 \mathrm{x}$ Tryptophan $(5 \mathrm{~g} / \mathrm{L})$

$50 \mathrm{ml} 40 \%$ Glucose or $40 \%$ Galactose

Up to 1 Liter $\mathrm{ddH}_{2} \mathrm{O}$ and use sterile filter

YPAD plate with Antibiotics (Clonat, $100 \mathrm{mg} / \mathrm{ml}$ )

$10 \mathrm{~g}$ Yeast Extract 
20 g Bacto-Pepton

15 g Bacto-Agar

Autoclave and add below ones after cooling down

$10 \mathrm{ml} \mathrm{100x}$ Adenine (4 g/L)

$50 \mathrm{ml} 40 \%$ Glucose

$1 \mathrm{ml}$ Clonat (final conc. $100 \mu \mathrm{g} / \mathrm{ml}$ )

Up to 1 Liter $\mathrm{ddH}_{2} \mathrm{O}$

YP.Gly/Lac. plate with iron chelators (Ferrozine, BPS)

$10 \mathrm{~g}$ Yeast Extract

20 g Bacto-Pepton

15 g Bacto-Agar

$37 \mathrm{ml}$ DL-Lactic acid (85\%)

$20 \mathrm{ml}$ Glycerol (final. 2\%)

pH 5.5 with approx. $30 \mathrm{ml} \mathrm{NaOH}$

Autoclave and up to 1 Liter $\mathrm{ddH}_{2} \mathrm{O}$

As for one plate with Ferrozine (final conc. 3, 1, 0.5 and $0.25 \mathrm{mM}$ )

Add $1 \mathrm{ml}$ to $0.25 \mathrm{ml} 1 \mathrm{M}$ Ferrozine as each final conc. for 1 Liter

BPS $(40 \mu \mathrm{M})$ SC plates were kindly provided by Kirsten Unthan-Fechner.

YP.Gly/Lac. plate with antibiotics (Rapamycin, $1 \mathrm{mg} / \mathrm{ml}$ )

$10 \mathrm{~g}$ Yeast Extract

20 g Bacto-Pepton

15 g Bacto-Agar

$37 \mathrm{ml}$ DL-Lactic acid

$20 \mathrm{ml}$ Glycerol (final. 2\%)

pH 5.5 with approx. $30 \mathrm{ml} \mathrm{NaOH}$

Autoclave and up to 1 Liter $\mathrm{ddH}_{2} \mathrm{O}$

As for one plate with Rapamycin (final conc. 4, 2, 1 and $0.5 \mathrm{nM}$ )

$3.7 \mu 1$ to $0.45 \mu 1$ per $1 \mathrm{ml}$ (total $12 \mathrm{ml} \mathrm{YP.Gly/Lac} \mathrm{for} 1$ plate)

Luria-Bertani (LB) Medium

$5 \mathrm{~g} / 1$ Peptone (Roth)

$2.5 \mathrm{~g} / 1$ Yeast extract (Roth)

$5 \mathrm{~g} / \mathrm{l} \mathrm{NaCl}$ (Roth)

$7.5 \mathrm{~g} / \mathrm{l}$ Agar (Roth), for plates

$100 \mu \mathrm{g} / \mathrm{ml}$ Ampicillin (Roth)

$15 \mu \mathrm{g} / \mathrm{ml}$ Kanamycin (Roth)

\section{Nematode Growth Medium (NGM)}

$16 \mathrm{~g} / \mathrm{l}$ Agar (Roth)

$3 \mathrm{~g} / \mathrm{l}$ Peptone (Roth)

$3 \mathrm{~g} / 1 \mathrm{NaCl}$ (Roth)

Add up to 1 Liter dd $_{2} \mathrm{O}$

Autoclave and add below ones after cooling down 
$25 \mathrm{ml} / 11 \mathrm{M} \mathrm{KPO}_{4}$ Solution (Roth)

$1 \mathrm{ml} / 11 \mathrm{M} \mathrm{MgSO}_{4}$ Solution (Roth)

$1 \mathrm{ml} / 11 \mathrm{M} \mathrm{CaCl}_{2}$ Solution (Roth)

$1 \mathrm{ml} / 110 \mathrm{mg} / \mathrm{ml}$ Nystatin (Sigma)

$1 \mathrm{ml} / 15 \mathrm{mg} / \mathrm{ml}$ Cholesterol (Roth)

NGM-N medium (nitrogen-free)

$3 \mathrm{~g} / \mathrm{l} \mathrm{NaCl}$ (Roth)

$12 \mathrm{~g} / 1$ Agarose (Roth or Invitrogen)

Add up to 1 Liter $\operatorname{ddH}_{2} \mathrm{O}$ and autoclave

Add below ones after cooling down

$25 \mathrm{ml} / 11 \mathrm{M} \mathrm{KPO}_{4}$ Solution (Roth)

$1 \mathrm{ml} / 11 \mathrm{M} \mathrm{MgSO}_{4}$ Solution (Roth)

$1 \mathrm{ml} / 11 \mathrm{M} \mathrm{CaCl}_{2}$ Solution (Roth)

$1 \mathrm{ml} / 110 \mathrm{mg} / \mathrm{ml}$ Nystatin (Sigma)

$1 \mathrm{ml} / 15 \mathrm{mg} / \mathrm{ml}$ Cholesterol (Roth)

M9 minimal growth medium (M9MM complete)

$100 \mathrm{ml} / 1$ 10x M9 salts

$5 \mathrm{ml} / 140 \%$ glucose

$1 \mathrm{ml} / 11 \mathrm{M} \mathrm{MgSO}_{4}$

$1 \mathrm{ml} / 11 \%$ Thiamine (wt/vol, final $0.01 \mathrm{~g} / \mathrm{L}$ )

Up to 1 Liter $\mathrm{ddH}_{2} \mathrm{O}$

Heavy-M9MM: Heavy amino acids (13C15N)

$1 \mathrm{ml} / 1$ of stock $0.2 \mathrm{M}$ heavy-Lysine in PBS

$1 \mathrm{ml} / 1$ of stock $0.2 \mathrm{M}$ heavy-Arginine in PBS

$1 \mathrm{ml} / 11 \mathrm{M}$ IPTG

$0.1 \mathrm{ml} / 110 \mathrm{mg} / \mathrm{ml}$ Carbenicillin

If needed, Biotine final conc. $50 \mu \mathrm{M}$ in solution (10 mM stock filter-sterilized)

Up to 1 Liter M9MM complete

Light-M9MM: Light amino acids (12C14N)

$1 \mathrm{ml} / \mathrm{l}$ of stock $0.2 \mathrm{M}$ light-Lysine in PBS

$1 \mathrm{ml} / \mathrm{l}$ of stock $0.2 \mathrm{M}$ light-Arginine in PBS

$1 \mathrm{ml} / 11 \mathrm{M} \mathrm{IPTG}$

$0.1 \mathrm{ml} / 110 \mathrm{mg} / \mathrm{ml}$ Carbenicillin

If needed, Biotine final conc. $50 \mu \mathrm{M}$ in solution (10 $\mathrm{mM}$ stock filter-sterilized)

Up to 1 Liter M9MM complete

D-Biotin Stock (10 mM, pH 7 9)

Use $50 \mu \mathrm{M}$ as a final conc. in M9MM for SILAC

Stock $10 \mathrm{mM}$ solution in $\mathrm{ddH}_{2} \mathrm{O}$

With constant stirring, add $1 \mathrm{M} \mathrm{NaOH}$ adjusted to $\mathrm{pH} 7 \sim 9$

Filter-sterilize into sterile bottle

$4^{\circ} \mathrm{C}$ and/or Store at $-20^{\circ}$

Table XI.4.2 Amino acids of selective media 


\begin{tabular}{lll}
\hline Amino acid & Resulting conc. per 1L (mg/L) & $\mathrm{mL}$ of 100x Stock $(\mathrm{g} / \mathrm{L})$ \\
\hline Adenine & 40 & 4 \\
Histidine & 20 & 2 \\
Leucine & 100 & 10 \\
Uracil & 20 & 2 \\
Methionine & 20 & 2 \\
Tryptophan & 50 & 5 \\
\hline
\end{tabular}

\subsection{Buffers and solutions}

Table XI.5.1 List of buffers and solutions

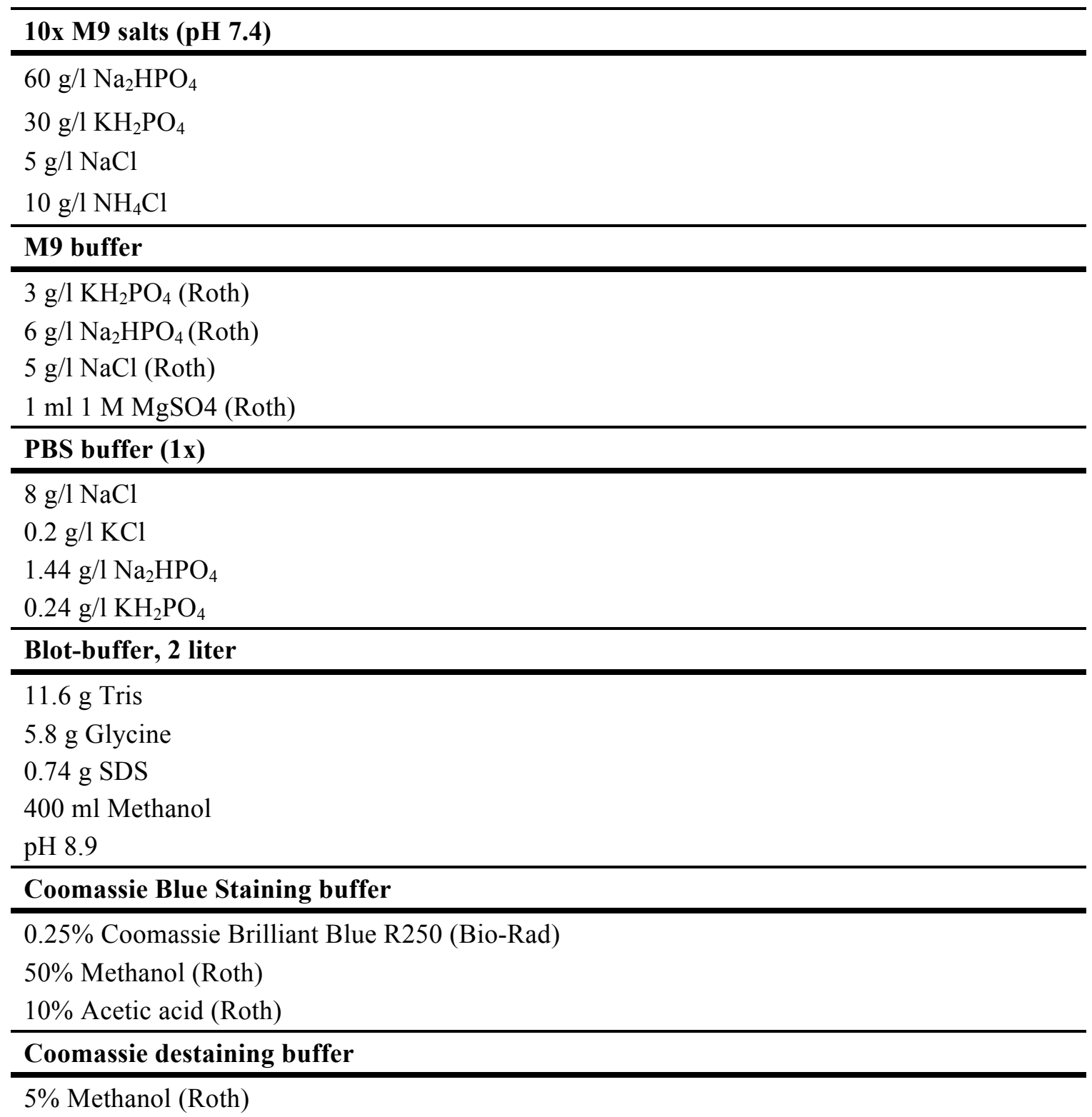


7.5\% Acetic acid (Roth)

Sample buffer for agarose gel loading (10x)

$30 \mathrm{ml} 100 \%$ Glycerol

$20 \mathrm{ml}$ EDTA $\mathrm{pH} 8.0$

$0.5 \mathrm{ml} 1 \mathrm{M}$ Tris $\mathrm{pH} 7.5$

A bit of bromphenol blue

$\mathrm{H}_{2} \mathrm{O}$ up to $50 \mathrm{ml}$

\section{S buffer}

$129 \mathrm{ml} 0.05 \mathrm{M} \mathrm{K}_{2} \mathrm{HPO}_{4}$

$871 \mathrm{ml} 0.05 \mathrm{M} \mathrm{KH}_{2} \mathrm{PO}_{4}$

\section{$5.85 \mathrm{~g} \mathrm{NaCl}$}

pH 6.0

Autoclave or filtrate

\section{SDS running buffer (10x)}

$30.3 \mathrm{~g} / 1$ Tris

$144 \mathrm{~g} / \mathrm{l} \mathrm{Glycine} \mathrm{(Roth)}$

$10 \mathrm{~g} / 1 \mathrm{SDS}$ (Roth)

$\mathrm{pH} 8.3$

\section{2x SDS sample buffer}

$121.1 \mathrm{~g} / 1$ Tris pH 6.8

$20 \%$ Glycerol (v/v)

$100 \mathrm{~g} / 1 \mathrm{SDS}$

$0.002 \%$ Bromophenol blue (w/v)

$200 \mathrm{ml}$ 2-Mercaptoethanol

\section{Solutions for developing Western blots}

Solution A (store in refrigerator, $4^{\circ} \mathrm{C}$ )

$200 \mathrm{ml} \mathrm{0.1M}$ Tris $\mathrm{HCl}$ (pH 8.6)

$50 \mathrm{mg}$ Luminol (Sigma)

Solution B (store in the dark)

$11 \mathrm{mg}$ para-hydroxycoumarin acid (dilute in $10 \mathrm{ml}$ DMSO)

$35 \% \mathrm{H}_{2} \mathrm{O}_{2}$

Prepare the mixture before use: $1 \mathrm{ml}$ Solution A, $100 \mu \mathrm{l}$ Solution B and $3 \mu \mathrm{H}_{2} \mathrm{O}_{2}(5 \mathrm{x} 8 \mathrm{~cm}$ for one nitrocellulose membrane, Protran ${ }^{\mathbb{B} .}$ Whatman $^{\mathbb{B}}$ ). Vortex the solution and distribute it onto the blot-membrane. Incubate for $2 \mathrm{~min}$ in the dark before developing.

TBE buffer (10x)

$108 \mathrm{~g} / \mathrm{l}$ Tris $\mathrm{NaOH}$

$55 \mathrm{~g} / 1$ Boric acid (Roth)

$9.3 \mathrm{~g} / 1$ EDTA $(\mathrm{pH} 8.0)$

\section{TE buffer}

$10 \mathrm{mM}$ Tris- $\mathrm{HCl} \mathrm{pH} 8.0$ 
1 mM EDTA (Roth)

Transformation and Storage Solution for chemical transformation (TSS)

$85 \%$ LB medium $(42.5 \mathrm{ml})$

$10 \%$ PEG (wt/vol, MW 8000) (5 g)

$5 \% \mathrm{DMSO}$ ( $\mathrm{vol} / \mathrm{vol})(2.5 \mathrm{ml})$

$50 \mathrm{mM} \mathrm{MgCl}_{2}$ (pH 6.5) (0.51 g)

Autoclave or filtrate and store at $4^{\circ} \mathrm{C}$.

Always prepare a fresh solution before preparing competent cells.

\section{Worm freezing solution}

S buffer $+30 \%$ glycerol

Worm lysis/PCR buffer (1x)

$100 \mathrm{mM}$ Tris $\mathrm{pH} 8.3$

$500 \mathrm{mM} \mathrm{KCl}$ (Roth)

$20 \mathrm{mM} \mathrm{MgCl}$ (Roth)

$20 \mathrm{mg} / \mathrm{ml}$ Proteinase K added fresh prior to lysis (Roth)

Worm lysis/PCR buffer (10x)

$1 \mathrm{M}$ Tris $\mathrm{pH} 8.3$

$5 \mathrm{M} \mathrm{KCl}$

$200 \mathrm{mM} \mathrm{MgCl}_{2}$

$1 \%$ Agarose gel

$1 \mathrm{~g}$ Agarose

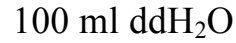

50\% PEG

500 g Polyethylglycol 3350

per 1 Liter $\mathrm{ddH}_{2} \mathrm{O}$

$10 \% \operatorname{SDS}(\mathrm{v} / \mathrm{v})$

$10 \mathrm{ml}$ SDS (sodium dodecyl sulfate)

per $100 \mathrm{ml} \mathrm{ddH} \mathrm{H}_{2} \mathrm{O}$

$10 \%$ APS (ammonium persulfate)

$1 \mathrm{~g}$ APS

$10 \mathrm{ml} \mathrm{ddH} \mathrm{H}_{2} \mathrm{O}$

\section{DTT (dithiothreitol)}

$3.86 \mathrm{~g}$ DTT in $\mathrm{ddH}_{2} \mathrm{O}, 25 \mathrm{ml}$ final volume for $1 \mathrm{M}$ stock

\subsection{Tris-HCl for separating gel}

118.2 g Tris- $\mathrm{HCl}, \mathrm{pH} 8.8$ for $500 \mathrm{ml}$ solution

\section{$1 \mathrm{M}$ Tris-HCl for stacking gel}

$78.8 \mathrm{~g}$ Tris- $\mathrm{HCl}, \mathrm{pH} 6.8$ for $500 \mathrm{ml}$ solution 


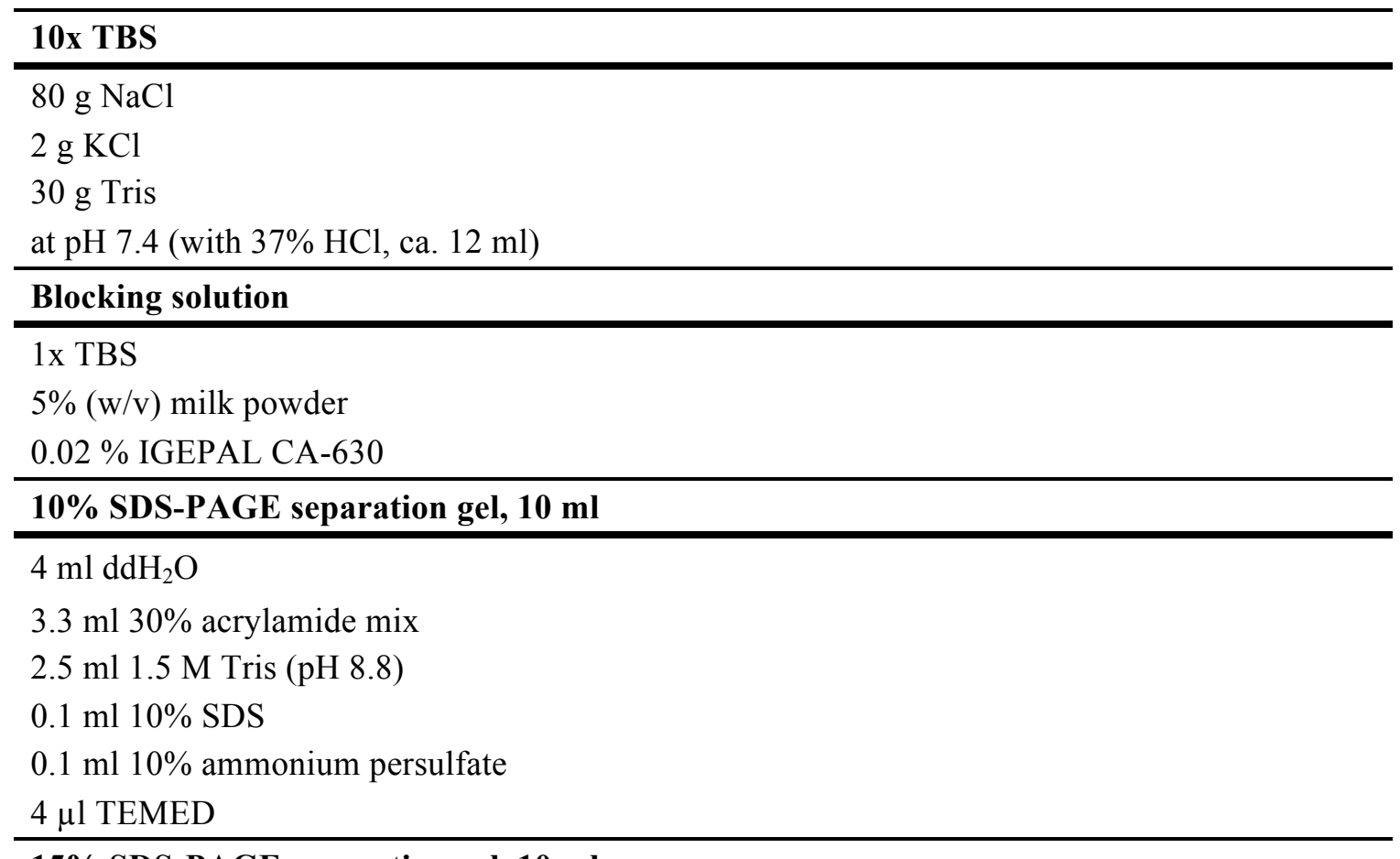

15\% SDS-PAGE separation gel, $10 \mathrm{ml}$

$2.3 \mathrm{ml} \mathrm{ddH} \mathrm{H}_{2} \mathrm{O}$

$5.0 \mathrm{ml} \mathrm{30 \%}$ acrylamide mix

$2.5 \mathrm{ml} 1.5 \mathrm{M}$ Tris (pH 8.8)

$0.1 \mathrm{ml} \mathrm{10 \%} \mathrm{SDS}$

$0.1 \mathrm{ml} \mathrm{10 \%}$ ammonium persulfate

$4 \mu 1$ TEMED

Stacking gel solution for $2 \mathrm{ml}$

$1.4 \mathrm{ml} \mathrm{ddH_{2 } \mathrm { O }}$

$0.33 \mathrm{ml} \mathrm{30 \%}$ acrylamide mix

$0.25 \mathrm{ml} 1.0 \mathrm{M}$ Tris (pH 6.8)

$0.02 \mathrm{ml} 10 \%$ SDS

$0.02 \mathrm{ml} \mathrm{10 \%} \mathrm{ammonium} \mathrm{persulfate}$

$2 \mu 1$ TEMED

10x Transfer buffer

$390 \mathrm{mM}$ Glycin, $480 \mathrm{mM}$ Tris $\mathrm{pH}$ 8.3, $3.7 \mathrm{~g}$ SDS

(add 20\% (v/v) Methanol in 1x buffer)

\section{5x SDS running buffer}

$250 \mathrm{mM}$ Glycin , $125 \mathrm{mM}$ Tris, 0.1\% (v/v) 10\% SDS, pH 8.3

5x SDS loading buffer

$250 \mathrm{mM}$ Tris- $\mathrm{HCl} \mathrm{pH} 6.8,5 \%$ (v/v) SDS, 0.5\% (w/v) BromphenolBlue, 50\% (v/v) Glycerol, $250 \mathrm{mM}$ DTT

20x TAE buffer 
800 mM Tris-HCl pH 7.5, 200 mM Sodium-Acetate, 20 mM EDTA pH 8.0

10x Ponceau S stock

$20 \mathrm{~g}$ Ponceau S, $300 \mathrm{~g}$ Trichloroacetic acid, $300 \mathrm{~g}$ Sulfosalicylic acid per 1 Liter $\mathrm{ddH}_{2} \mathrm{O}$

Table XI.5.2 Antibiotic concentrations used in this study

\begin{tabular}{ll}
\hline Working concentration & Antibiotics (Stock, -20 $\left.{ }^{\circ} \mathbf{C}\right)$ \\
\hline $100 \mu \mathrm{g} / \mathrm{ml}$ & Clonat $(100 \mathrm{mg} / \mathrm{ml})$ \\
\hline $2-0.5 \mathrm{nM}$ & Rapamycin $(1 \mathrm{mg} / \mathrm{ml})$ \\
\hline $200 \mu \mathrm{g} / \mathrm{ml}$ & G418 (Kan) $(200 \mathrm{mg} / \mathrm{ml})$ \\
\hline $1 \mu \mathrm{g} / \mathrm{ml}$ & Carbenicillin $(10 \mathrm{mg} / \mathrm{ml})$ \\
\hline $100 \mu \mathrm{g} / \mathrm{ml}$ & Ampicillin $(50 \mathrm{mg} / \mathrm{ml})$ \\
\hline $12.5 \mu \mathrm{g} / \mathrm{ml}$ & Tetracycline $(12.5 \mathrm{mg} / \mathrm{ml})$ \\
\hline $25 \mu \mathrm{g} / \mathrm{ml}$ & Kanamycin $(25 \mathrm{mg} / \mathrm{ml})$ \\
\hline
\end{tabular}

\subsection{Materials and reagents}

\subsubsection{Research equipment used in this study}

Table XI.6.1 Laboratory equipment (Equipment / Manufacturer)

\begin{tabular}{ll}
\hline Centrifuges & Company \\
\hline 4 K15 & SIGMA \\
$5415 D$ & Eppendorf \\
Biofuge pico & Heraeus \\
Centrifuge 5424 & Eppendorf \\
Mini-centrifuge & Eppendorf \\
Microcentrifuge 5417 & Eppendorf \\
5810 R and rotor A-4-62 & Eppendorf \\
\hline Microscopes & \\
\hline Delta Vision RT microscope & Applied Precision \\
ZEISS Axioskop & ZEISS \\
LSM 510 (inverted) & ZEISS \\
LSM 510 META UV (inverted) & ZEISS \\
TCS SP8 STED (inverted) & LEICA \\
TCS SP2 (inverted) & Leica \\
BZ-9000 (BIOREVO) & Keyence \\
Stereo Microscope system & Olympus \\
SZX9 Fluorescence Stereomicroscope & Olympus \\
MZ16 FA, Light source: KL2500 LCD & Leica
\end{tabular}




\begin{tabular}{|c|c|}
\hline MZ6 Stereo Microscope & Leica \\
\hline \multicolumn{2}{|l|}{ Power suppliers and pouring machines } \\
\hline $\begin{array}{l}\text { PowerPAC }{ }^{\mathrm{TM}} \text { Basic } \\
\text { PowerPAC }{ }^{\mathrm{TM}} 200 \\
\text { Power supply EV231 } \\
\text { Power supply EV213 } \\
\text { Power supply } \\
\text { Plate pouring pump MCP } \\
\text { PourMatic MP-1000 }\end{array}$ & $\begin{array}{l}\text { Bio-Rad } \\
\text { Bio-Rad } \\
\text { Consort } \\
\text { Consort } \\
\text { Invitrogen } \\
\text { ISMATIC } \\
\text { New Brunswick Scientific GmbH }\end{array}$ \\
\hline \multicolumn{2}{|l|}{ Incubators and Shakers } \\
\hline $\begin{array}{l}\text { Innova } 4230 \\
\text { Innova } 44 \\
\text { Worm incubator MPR- } 1410 \\
\text { Incubators for } 30^{\circ} \mathrm{C} \text { and } 37^{\circ} \mathrm{C} \\
\text { Block heater SBH130 } \\
\text { Heat Block: DRI-BLOCK }{ }^{\circledR} \text { DB-2D } \\
\text { Shaker: ST5 } \\
\text { Waterbath Lauda EcoLine } 003 \text { E100 } \\
\text { FUNCTION Line } \\
\text { Orbital shaker for } 37^{\circ} \mathrm{C} \\
\text { Orbital shaker Rotamax } 120 \\
\text { Thermomixer comfort }\end{array}$ & $\begin{array}{l}\text { New Brunswick Scientific GmbH } \\
\text { New Brunswick Scientific GmbH } \\
\text { Sanyo } \\
\text { Memmert and Binder } \\
\text { Stuart } \\
\text { TECHNE } \\
\text { CAT } \\
\text { Gemini BV Laboratory } \\
\text { Heraeus } \\
\text { New Brunswick Scientific GmbH } \\
\text { Heidolph, Germany } \\
\text { Eppendorf }\end{array}$ \\
\hline \multicolumn{2}{|l|}{ Measuring machines } \\
\hline $\begin{array}{l}\text { NanoDrop1000 and } 2000 \\
\text { Spectrophotometry Biomate5 } \\
\text { Odyssey } \\
\text { Precision scale and easy-use scale } \\
\text { pH-Meter WTW Series } \\
\text { pH-Meter } 766 \text { calimatic } \\
\text { BioPhotometer } \\
\text { UV systems for DNA gel pictures } \\
\text { LAS3000 and } 4000 \\
\text { Extend ED224S and ED4202S } \\
\text { Libra S22 }\end{array}$ & $\begin{array}{l}\text { Thermo Scientific } \\
\text { Thermo Scientific } \\
\text { LI-COR } \\
\text { Kern\&Sohn GmbH } \\
\text { InoLab } \\
\text { Knick } \\
\text { Eppendorf } \\
\text { INTAS } \\
\text { Fujifilm } \\
\text { Satorious } \\
\text { Biochrom }\end{array}$ \\
\hline \multicolumn{2}{|l|}{ Injection machine } \\
\hline $\begin{array}{l}\text { Microscope Axiovert } 200 \\
\text { Pump Eppendorf FemtoJet } \\
\text { Micro manipulator }\end{array}$ & $\begin{array}{l}\text { Zeiss } \\
\text { Eppendorf } \\
\text { Leica }\end{array}$ \\
\hline \multicolumn{2}{|l|}{ Electrophoresis systems and others } \\
\hline $\begin{array}{l}\text { Mini-PROTEAN }{ }^{\circledR} 3 \text { system } \\
\text { Horizontal electrophoresis system unit } \\
\text { Semi-Dry-Blotter } \\
\text { MasterCycler EP S PCR thermal cyclers } \\
\text { Sonicator UP200S } \\
\text { SpeedVac } 5301 \text { concentrator } \\
\text { Magnetic Stirrer MR3001 } \\
\text { Magnetic Stirrer: IKAMAG }{ }^{\circledR} \text { RET } \\
\text { Disruptor Genie }\end{array}$ & $\begin{array}{l}\text { Bio-Rad } \\
\text { PEQLAB Biotechnologie GmbH } \\
\text { Phase } \\
\text { Eppendorf } \\
\text { Hielscher } \\
\text { Eppendorf } \\
\text { Heidolph } \\
\text { Fisher Scientific } \\
\text { Scientific Industries }\end{array}$ \\
\hline
\end{tabular}




\subsubsection{Chemicals}

Table XI.6.2 Chemicals (Chemicals / Manufacturer) used in this study

\begin{tabular}{|c|c|}
\hline Chemicals & Company \\
\hline $20 \%$ SDS Solution & AppliChem \\
\hline $30 \%$ acrylamide mix & AppliChem \\
\hline Adenine & SIGMA \\
\hline Adeninsulfate & AppliChem \\
\hline Agar Kobe I & AppliChem \\
\hline Agarose (Biochemistry, Uni-Göttingen) & PEQLAB \\
\hline Agarose & Invitrogen \\
\hline Alanine & AppliChem \\
\hline Ampicillin & Roth \\
\hline APS (ammonium persulfate) & BIOMOL \\
\hline Arginine & AppliChem \\
\hline Asparagine & AppliChem \\
\hline Bacto-Agar & $\mathrm{BD}$ \\
\hline Bacto-Pepton & $\mathrm{BD}$ \\
\hline Boric acid & Roth \\
\hline BPS (Batho Phenantroline diSulfate) & SIGMA \\
\hline Bromphenol blue & Roth \\
\hline Carbenicillin & Roth \\
\hline Chloramphenicol & Roth \\
\hline Cholesterol & Roth \\
\hline CHX (Cycloheximides) & SIGMA \\
\hline Clonat (nourseothricin) & WERNER BioAgents \\
\hline Coomassie Brilliant Blue R250 stain & Bio-Rad \\
\hline CSM amino acid powders w/o URA & Mpbio \\
\hline CSM-ADE-HIS-LEU-MET-TRP-URA & Mpbio \\
\hline D-Latic Acid & SIGMA \\
\hline Dithiothreit (DTT) & Roth \\
\hline DL-Lactic acid (85\%) & SIGMA \\
\hline DMEM & PAA \\
\hline DMSO (Dimethysulfoxide) & SIGMA \\
\hline DTT (dithiothreitol) & SIGMA \\
\hline EDTA (Ethylenediaminetetraaceticacid) & AppliChem \\
\hline Ethanol absolute $100 \%$ & Roth \\
\hline Ethidium bromide $1 \%$ & Roth \\
\hline Ferrozine & SIGMA \\
\hline G418 (Kanamycin) & SIGMA \\
\hline Glass beads, acid-washed 710-1,180 $\mu \mathrm{m}$ & SIGMA \\
\hline Glucose & Roth \\
\hline
\end{tabular}


Glutamic Acid

Glutamine

Glutathione

Glycerol

Glycerol 87\%

Glycerol 99.9\%

Glycine

Histidine Monohydrochloride

Hydrochloric acid 32\%

Hydrogen peroxide

hydrogenphosphate

IGEPAL CA-630

Imidazole

Immersion oil

IPTG

Isoleucine

Isopropanol

Kanamycin

$\mathrm{KCl}$ (potassium chloride)

L-arginine

L-lysine

L-arginine:HCL (U-13C6, U-15N4)

L-lysine:2HCL (U-13C6, U-15N2)

LB media

Leucine

Lithium acetate

Lithium chloride

Luminol

Mercaptoethanol

Methionine

Milk powder

Milk powder low fat

N-Ethylmaleimide (NEM)

$\mathrm{NaCl}$ (sodium chloride)

$\mathrm{NaOH}$ (sodium hydroxide)

Nystatine

PEG (Polyethylene glycol) 3350

PEG (Polyethylene glycol) 4000

Peptone

Pefabloc protease inhibitor cocktail

Phenylalanine

Polyethylene glycol PEG

Ponceau S
AppliChem

AppliChem

Roth

Roth

AppliChem

University of Göttingen

Roth

Sigma

Roth

Roth

Roth

SIGMA

Roth

Roth

Roth

AppliChem

Roth

Roth

Merck

AppliChem

AppliChem

Cambridge Isotope Laboratories, Inc.

Cambridge Isotope Laboratories, Inc.

Roth

Sigma

Roth

Roth

Sigma

Sigma

AppliChem

Roth

Rewe

Sigma

Roth

Roth

AppliChem

SERVA

SERVA

Roth

Roche

AppliChem

Roth

AppliChem 
Potassium chloride

Powdered guanidine- $\mathrm{HCl}$

Prefabloc SC-Protease Inhibitor

Proline

Rapamycin

Rotiphorese gel

SDS (sodium dodecyl sulfate)

SDS pellet

Select Agar

Serine

Sodium azide

Sodium chloride

Sodium dihydrogenphosphate di-sodium

Sodium hydroxide

Sodium hydroxide $32 \%$

Sodium hypochloride $12 \%$

Sodium sulfate

TEMED

Threonine

Tris

Tris (2-carboxyethyl) phosphine $\mathrm{HCl}$ (TCEP)

Tris- $\mathrm{HCl}$

Triton X 100

Tryptophan

Tween 20

Tyrosine

UltraPure Agarose

Uracil

Valine

Yeast Extract

Yeast extract

Yeast Nitrogen Base
Roth

Roth

Roth

AppliChem

SIGMA

Roth

AppliChem

Roth

Invitrogen

AppliChem

Sigma

Roth

Roth

Roth

Roth

Roth

Roth

Roth

AppliChem

Roth

Sigma

AppliChem

Roth

Sigma

Roth

AppliChem

Invitrogen

Fluka

AppliChem

AppliChem

Roth

Invitrogen

Melford

Other chemicals used in this study were manufactured by Sigma, Roth, Roche, Serva, Merck, USB, AppliChem, J.T.Baker, Boehringer, Biomol, Amersham, Molecular Probes and Bio-Rad and supported by Prof. Dr. Stefan Eimer (ENI, University of Göttigen and Bioss, University of Freiburg) and Prof. Dr. Blanche Schwappach, Biochemistry I, Universitätsmedizin Göttingen, Germany.

\subsubsection{Enzymes and kits}

Table XI.6.3 Enzymes or Kits (Kits / Supplier)

\begin{tabular}{ll}
\hline Kits & Supplier \\
\hline dNTP Set, $100 \mathrm{mM}$ Solutions & Fermentas
\end{tabular}


YeaStar ${ }^{\mathrm{TM}}$ Genomic DNA Kit

Nucleic Acid and protein purification

NucleoSpin ${ }^{\circledR}$ Plasmid

High pure PCR product purification kit

Restriction Enzymes

T4 DNA Ligase

GeneRuler ${ }^{\mathrm{TM}} 1 \mathrm{~kb}$ DNA ladder

PageRuler ${ }^{\mathrm{TM}}$ Prestained Protein Ladder

6x DNA loading buffer

Expand High Fidelity (Taq, 10x buffer)

PfuUltra II Fusion HS DNA Polymerase

TaqA Polymerase

pTC Polymerase

SafeView ${ }^{\text {TM }}$ Nucleic acid stain

LysoTracker ${ }^{\circledR}$ Red DND-99

$\mathrm{FM}^{\circledR}$ 4-64

Mini-prep Kit

Gel Extraction Kits

PCR purification Kits

Proteinase K

Complete protease inhibitor mini-tablets

PhosStop

Roti ${ }^{\mathbb{B}}$-Quant

$4-15 \%$ Mini-PROTEAN ${ }^{\circledR}$ TGX $^{\text {TM }}$ Gel

Dynabeads MyOne C1
Zymo Research

MACHEREY-NAGEL

MACHEREY-NAGEL

Roche

Fermentas

Fermentas

Fermantas

Thermo Scientific

Fermentas

Roche

Stratagene

Lab-made, recombinant

Lab-made, recombinant

Applied Biological Materials Inc.

Molecular Probes ${ }^{\circledR}$

Molecular Probes ${ }^{\circledR}$

Invitrogen

Fermentas

Fermentas

Roth

Roche

Roche

Roth

Bio-Rad

Invitrogen

\subsubsection{Antibodies}

\section{Table XI.6.4 List of antibodies used in this study}

\section{Primary antibodies, 1:1000 dilution}

anti-GFP mouse monoclonal clone 3E6 $(0.2 \mathrm{mg} / \mathrm{ml}) /$ Invitrogen

anti-GFP mouse monoclonal clone 7.1 and $13.1(0.4 \mathrm{mg} / \mathrm{ml}) /$ Roche

anti-GFP mouse monoclonal B-2 $(0.2 \mathrm{mg} / \mathrm{ml}) /$ Santa Cruz

Living Colors ${ }^{\circledR}$ DsRed polyclonal antibody $(1 \mathrm{mg} / \mathrm{ml}) /$ Clontech Laboratories, Inc

anti-PGK1 mouse monoclonal 22C5 $(1 \mathrm{mg} / \mathrm{ml}) /$ Molecular Probes, Invitrogen

anti-Bmh1p rabbit (1:1000)/ Schwappach lab (Michelsen et al., 2006)

anti-c-myc mouse (1:2000), $200 \mathrm{ng} / \mathrm{ml}$ (provided from Stefan Eimer)

\section{Secondary antibodies, $1: 10,000$ dilution}

IRDye ${ }^{\circledR} 680$ LT Infrared Dye-conjugated secondary anti-rabbit or anti-mouse, LI-COR

IRDye ${ }^{\circledR} 800 \mathrm{CW}$ Infrared Dye-conjugated secondary anti-rabbit or anti-mouse, LI-COR

Peroxidase-conjugated AffiniPure anti-mouse IgG $0.8 \mathrm{mg} / \mathrm{ml}$ anti-goat, Jackson

Peroxidase-conjugated AffiniPure anti-rat IgG $0.8 \mathrm{mg} / \mathrm{ml}$ anti-goat, Jackson

Peroxidase-conjugated AffiniPure anti-rabbit IgG $0.8 \mathrm{mg} / \mathrm{ml}$ anti-goat, Jackson

Streptavidin-HRP, 1:4000, 1mg/ml, Thermo Scientific 


\section{CURRICULUM VITAE}

\section{PERSONAL INFORMATION}

Name

Suekyoung Jeon

Date of birth

10 October 1977

Place of birth

Seoul, South Korea

Address

Zähringer Str. 361, 79108 Freiburg im Breisgau

Nationality

Republic of Korea (South Korea)

Email suekyoung.jeon@stud.uni-goettingen.de

EDUCATION

Project of PhD thesis

Dec. 2013 to date

Feb. 2011 - Nov. 2013

\section{Feb. 2011 - 2014}

Centre for Biological Signalling Studies, University of Freiburg, Germany

European Neuroscience Institute, University of Göttingen, Germany

\section{Master of Science in Microbiology}

Mar. 2000 - Feb. 2002 Microbiology, Konkuk University, South Korea

Master's Thesis: Characteristics of the Molecular Genetics for env (V1-C5) Gene isolated from Asymptomatics and AIDS Patients with HIV-1 Korean Subtype

\section{Bachelor of Science in Molecular Biology}

Mar. 1995 - Feb. 2000 Molecular Biology, Konkuk University, South Korea

\section{SCHOLARSHIPS AND RESEARCH SUPPORTS}

Dec. 2013 - At present Centre for Biological Signalling Studies, Universität Freiburg

Feb. 2011 - Nov. 2013 SyMBaD International Training Program, EU consortium

\section{WORK EXPERIENCE}

May 2013 - At present

Center for Biological Systems Analysis, Freiburg, Germany

Nov. 2012 - Apr. 2013 Biochemistry I, Universitätsmedizin Göttingen, Germany

Feb. 2011 - Oct. 2012 European Neuroscience Institute, Göttingen, Germany

Feb. 2003 - Mar. 2010 Beckman Coulter Korea Ltd. and BKI Inc., South Korea

Sep. 2000 - Jan. 2003 National Institute of Health, Seoul, South Korea 


\section{LIST OF PUBLICATIONS}

Nam JG, Kim KC, Jeon SK, Kim SS, Lee SW, An SW, Lee JS. Immunodeficiency Virus

Type 1 Infection of H9 Cells. Journal of Bacteriology and Virology, 2004 Mar;34(1):5766. The Korean Society for Microbiology. 